


\section{Bioengineering of pre-vascularized bone tissue analogues}

Laying the basis for a perfusable hierarchical vascular supply 
ISBN number: 978-94-6380-853-8

Cover design: K. Godfried

Printed by: ProefschriftMaken || www.proefschriftmaken.nl

Copyright $\odot$ Iris Pennings, the Netherlands, 2020. All rights reserved. No part of this thesis may be reproduced, distributed, stored in a retrieval system of any nature or transmitted in any form of by any means, without the written consent of the author or, when appropriate, the publisher of the respective publications.

This research was partially funded by the Nederlandse Organisatie voor Wetenschappelijk Onderzoek (NWO). Financial support for the printing of the thesis was kindly provided by the Dutch society for Biomaterials and Tissue Engineering (NBTE) and the 'Utrechtse Stichting tot Bevordering der Mondziekten, Kaak- en Aangezichtschirurgie'. 


\section{Bioengineering of pre-vascularized bone tissue analogues}

Laying the basis for a perfusable hierarchical vascular supply

De ontwikkeling van gevasculariseerde botanalogen

Het leggen van de basis voor een perfuseerbare hiërarchische vasculaire structuur

(met een samenvatting in het Nederlands)

\section{Proefschrift}

ter verkrijging van de graad van doctor aan de

Universiteit Utrecht

op gezag van de

rector magnificus, prof.dr. H.R.B.M. Kummeling,

ingevolge het besluit van het college voor promoties

in het openbaar te verdedigen op

donderdag 29 oktober 2020 des ochtends te 11.00 uur

door

\section{Iris Pennings}

geboren op 16 augustus 1992

te Rosmalen 


\section{Promotoren:}

Prof. dr. A.J.W.P. Rosenberg

Prof. dr. M.C. Verhaar

\section{Copromotoren:}

Dr. ir. D. Gawlitta

Dr. C. Cheng 
The essence of knowledge is, having it, to apply it; not having it, to confess your ignorance

(Confucius) 



\section{Table of content}

Chapter 1 General introduction 9

Part I Bioengineering of macroscale vessels 23

Chapter 2 Heterotypic scaffold design orchestrates primary cell organization and phenotypes in co-cultured small diameter vascular grafts

Chapter 3 Layer-specific cell differentiation in bi-layered vascular grafts under flow perfusion

Part II Fabrication of microscale vasculature in osteogenic constructs

Chapter 4 Effect of donor variation on osteogenesis and vasculogenesis in hydrogel co-cultures

Chapter 5 In vitro pre-differentiation of prevascularized bone constructs containing calcium phosphates accelerates the onset of in vivo bone formation

Chapter 6 Osteogenic stimulation interferes with the maintenance of capillary-like networks in osteogenic constructs

Chapter 7 Summary and general discussion

Appendix 1 Arteriovenous loop model for vascularization of bone tissues

Appendix 2 In vitro interconnectivity of multi-level vasculature

References

Nederlandse samenvatting

List of publications

Dankwoord 


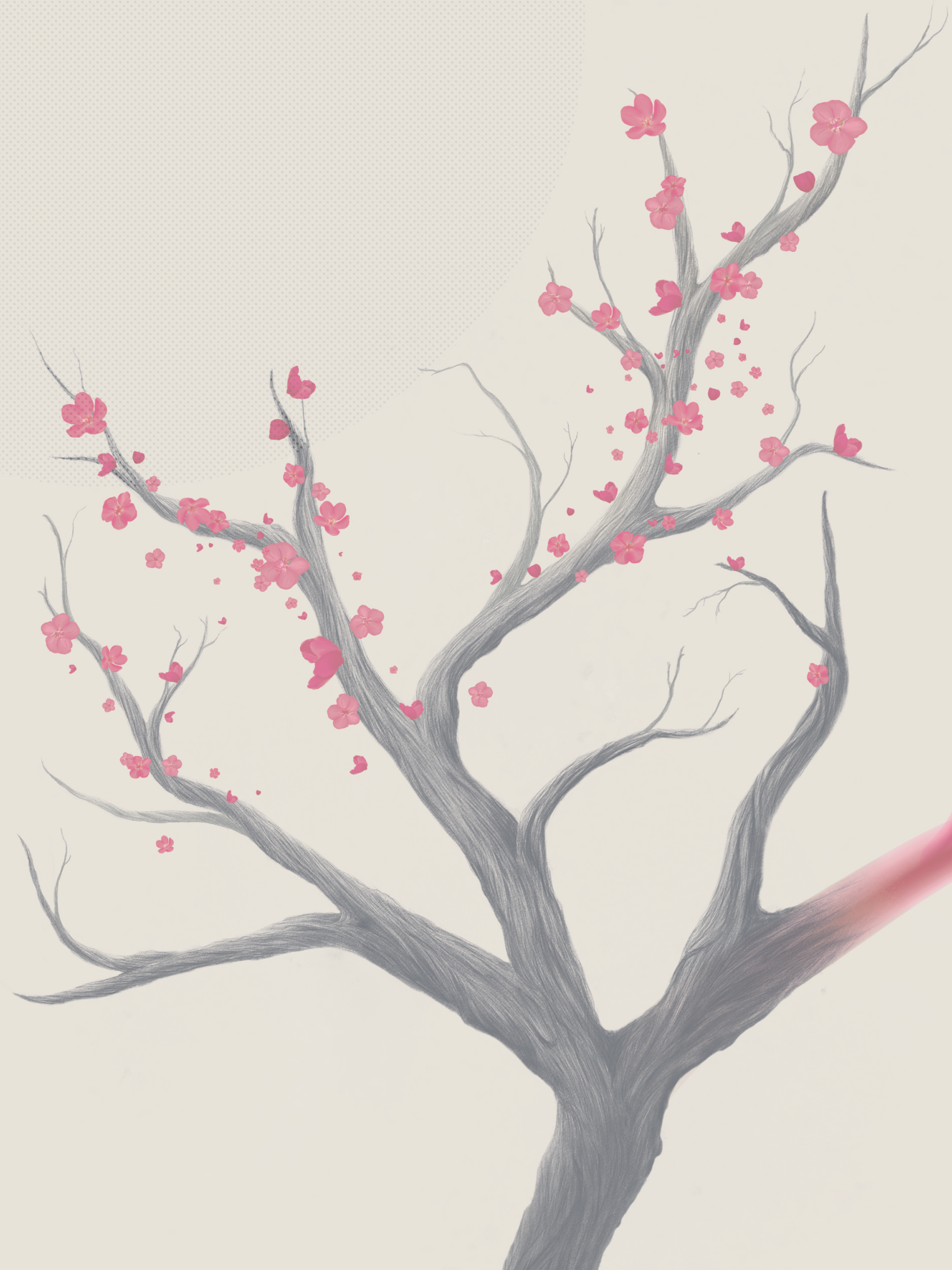




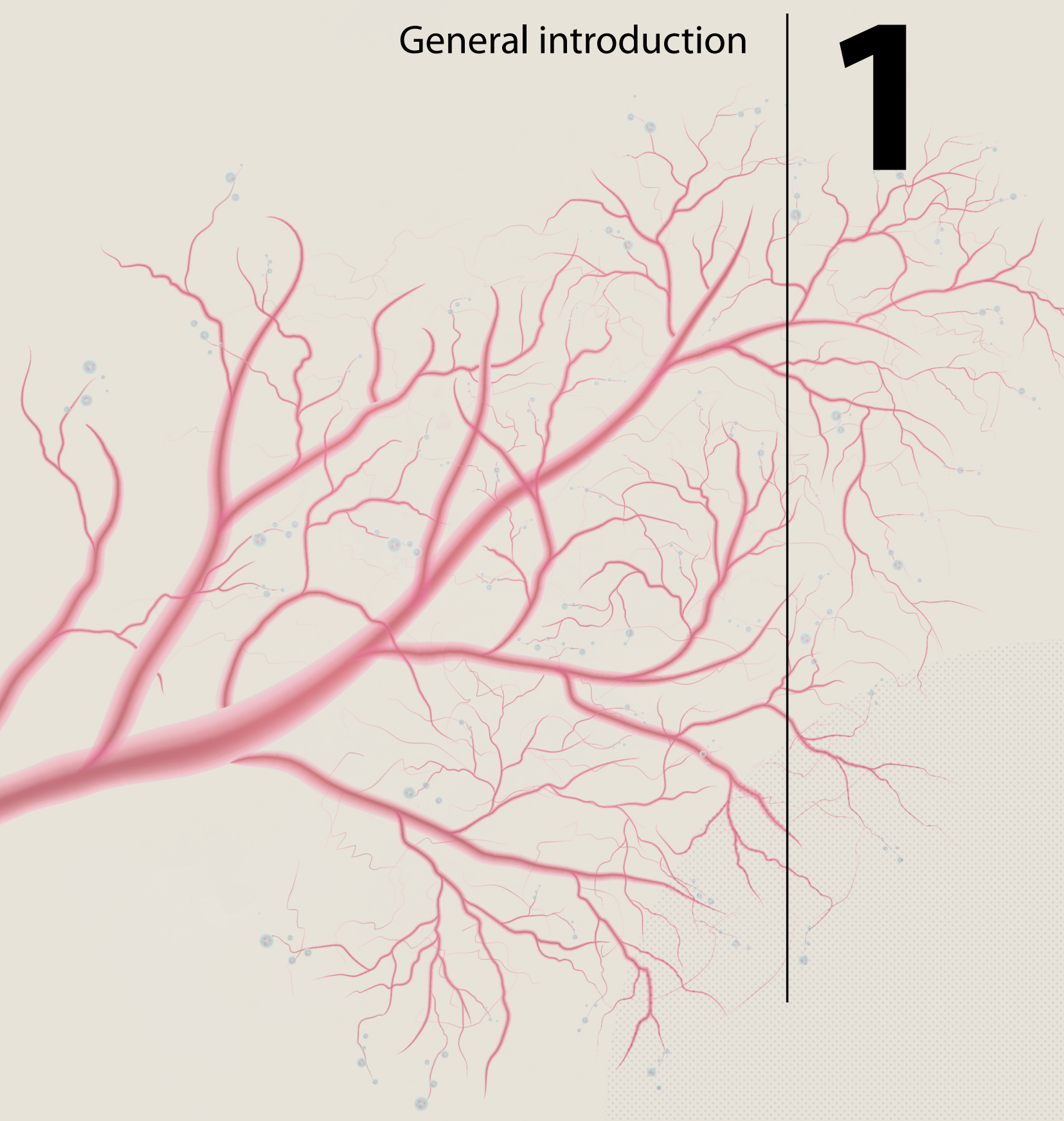




\section{Preface}

In the human body there is a constant state of regeneration for the renewal of our tissues. Traditionally, regeneration is defined by the ability to regrow a damaged or missing organ from the remaining tissue. But it can also be as simple as the growth of nails or the restoration of your skin after a sunburn. The interplay of regeneration and degeneration sustains a life-long dynamic renewal of the body, tailored to, for example, (fetal) development (e.g., development of the limbs), adaptions to changes in the environment (e.g., bone remodeling) and healing of injured tissues (e.g., skin lesion) [1]. Some tissues heal better and faster than others, where the liver and skin are traditionally considered as those with the highest regenerative capacity, while bone is a tissue that only has an intermediate regenerative capacity [2]. Nevertheless, independent of the regenerative capacity of the tissue, if the degeneration exceeds a certain threshold, for example, a too big injury, the body won't be able to heal itself and therapeutic interventions are required. In the craniomaxillofacial area specifically, this can be caused by trauma, tumor resection, radiotherapy, infections or congenital deformities, which can lead to defects in the mandibular region (lower jaw bone) beyond repair [3]. Traditional therapies are aimed towards replacing the damaged tissue by implanting inert materials (e.g., metal implants), or replacing the diseased tissue with donor material (allogenic) or material from a donor site from the patient's own body (autologous). However, these treatments do not aim for truly regenerating the diseased bone tissue via the body's own capacity.

In the last decades, the field of regenerative medicine has advanced rapidly and aims to develop therapeutic interventions that assist the body to restore the function of an injured tissue, either via self-healing or via tissue engineering (TE) [4]. TE is 'a science devoted to the generation of new tissue by employing the principles of engineering in combination with the application of certain biologic principles' [5]. Generally this field combines bioactive cues, scaffolding materials and/or cells to develop functional tissues or to promote endogenous regeneration $[5,6]$. Advances in the field of biology, biomaterials and biofabrication methods have already contributed to the development of bioengineered bone constructs [7], and moreover, have provided fundamental knowledge on bone regeneration and its dependence on the growth of blood vessels (i.e., angiogenesis) [8].

This thesis is focused on the development of a bone construct, that is combined with different types of blood vessels to ensure a correct integration and blood perfusion once implanted in patients. This chapter is an introduction to bone tissue and blood vessels, and indicates how these tissues combined can contribute to the further development of biological implants for patients with critical size defects in bone areas (e.g., mandibular defects). 


\section{Bone tissue}

Bone is an organ that provides for mobility, soft organ protection, storage of minerals and offers a bone marrow cavity for hematopoiesis [7]). When looking specifically at the craniomaxillofacial area, it consists of cranial bones and facial bones. The cranial bones enclose and protect the brain whereas the facial bones, such as the maxilla and mandible, serve as structural support for the dental region and are necessary for talking, eating, breathing and separating the oral, orbital and nasal cavities [9]. Besides the functionality of these bones, there is another matter that is determined by the craniomaxillofacial structures, which is the one most prominently visible: our esthetics. This makes the surgical reconstruction of maxillofacial defects a complex procedure, as both functional and esthetic challenges must be addressed. This stresses the need for adequate therapies.

Bone tissue is a vascularized tissue consisting of cells and a mineralized extracellular matrix (ECM) [10]. It is a dynamic tissue with a turnover of approximately $100 \%$ in the first year of life, declining to below $10 \%$ per year throughout the rest of life [11-14]. This turnover is the result of a process called bone remodeling, a necessity to maintain mineral homeostasis, skeletal stability and integrity, and can be a response to changing demands in mechanical loading. Remodeling occurs by bone remodeling units (BRU) through a series of events that involves an interplay between osteoclast-facilitated bone resorption and new bone formation (osteogenesis) by osteoblasts [14-16]. Osteoclasts are multinucleated cells of hematopoietic origin that affix themselves to the bone matrix and degrade the inorganic constituents via an acidic solution. After the resorption, the process of osteogenesis starts when osteoblasts are recruited and activated to lay down a layer of soft ECM, composed of collagen type I and other non-collagenous proteins (e.g., osteocalcin and osteonectin); collectively known by the comprehensive term 'osteoid' $[14,16]$. Subsequently, osteoblasts mineralize this matrix by the deposition of mineral crystals on the collagen bundles via the activity of an enzyme called alkaline phosphatase (ALP) [17]. During this process, osteoblasts that were once lining the new bone become entrapped in the matrix and transform into osteocytes. These osteocytes then secrete factors (e.g., sclerostin) that signal to the residual osteoblasts to become quiescent and to form a flattened line of cells at the bone surface $[10,16]$. They persist there until a new cycle of bone remodeling is initiated by the random recruitment of mononuclear osteoclast precursors or by targeted recruitment via osteocyte signaling.

During bone development, osteogenesis can occur via two processes: endochondral ossification or intramembranous ossification. Endochondral ossification describes a process in which an intermediary cartilaginous template is first formed by chondrocytes, ossified and later on remodeled into bone tissue by osteoclasts and osteoblasts. In contrast, in the process of intramembranous ossification, bone is directly formed by 
the differentiation of progenitor cells into osteoblasts. Both processes are also involved in normal fracture healing after injury, either individually or in combination, with the endochondral pathway being the most prominent one [15]. In cases where the defects are not able to heal via natural processes, we speak of critical size defects. In such cases, surgery is inevitable and interventions for bone substitute (graft) implantation are a requisite [15]. In the craniomaxillofacial surgical specialty, the choice of treatment and type of graft is mostly reliant on the size and cause of the defect, mediating if the treatment can be resolved via non-vascularized bone grafts (defects approximately < 6 $\mathrm{cm}$ ) or via autologous vascularized bone transplants for larger and longer defects [3]. Nonvascularized substitutes are often derived from synthetic materials or from bone chips or blocks originating from the iliac crest, and their integration depends on the ingrowth of vasculature from the surrounding tissue [18]. Modalities to treat larger defects include the use of an autologous vascularized bone flap (e.g., from the radius or fibula), ensuring an immediate perfusion of the graft after transplantation $[3,19,20]$. The existence of an intact vasculature ensures the preservation of mechanical strength, tissue viability and structural integrity of the graft after transplantation (especially after radiation therapy of the acceptor bed) $[18,21]$. However, availability of such grafts is limited and graft harvesting is associated with donor site morbidity.

\section{Regenerative therapies for bone replacement}

The introduction of such new skeletal defects and associated complications at the donor site, as well as size mismatch, can be resolved by developing patient-specific bone constructs. This can be achieved by making use of the principles of regenerative medicine. Regenerative therapies for bone tissue engineering (BTE) have been a widely studied topic with focus on several key players, including the choice of growth factors (e.g., bone morphogenetic protein (BMP), vascular endothelial growth factor (VEGF), fibroblastic growth factor (FGF), platelet derived growth factor (PDGF) and transforming growth factor $\beta$ (TGF- $\beta$ )), biomaterials (e.g., titanium, bioactive glass, calcium phosphate (CaP) ceramics, hydrogels, polymers or ion substitutions) and cells (e.g., osteoblasts or cordblood/bone marrow (BM)/adipose/dental pulp derived stem cells) [7, 22-24]. Although many BTE strategies have been investigated, approval for clinical applications has only been obtained for a few of them. To date, the accepted strategies mostly involve single component (growth factors, scaffolds or cells individually) or dual component strategies $[7,25]$. Some examples of BTE products on the market are: PDGF, BMP2 or BMP7 infused scaffolds (Augment bone graft, BioMimitic therapeutics; Infuse, Medtronic; OP-1, Olympus Biotech), CaP ceramic based scaffolds (ACTIFUSE, Baxter; NovaBone Putty, mtfbiologics; Conduit, Orthopaedics Inc) and bioactive glasses (Vitos, Orthovita). Additionally, some clinical trials for cell-based BTE products were performed, including but not limited to 'same-day isolation' of BM-derived cells (Regenexx, Regenerative Sciences), autologous BM-derived osteoprogenitor cells with demineralized allograft bone matrix (Osteocel Plus, 
NuVasive) and a multi-component strategy of an allogenic bone matrix combined with osteoblasts, multipotent mesenchymal stromal cells (MSCs) and growth factors (VEGF, FGF and PDGF) ( $\mathrm{BIO}^{4}$, Osiris Therapeutics) [26]. Despite the bone regenerating capacity of these BTE products on the market, it should be noted that difficulties with powerful local or paracrine effects of growth factors, safety issues and FDA approval of cell-based therapies, and restriction of scaffolds to non-load bearing defects are challenges still being faced $[7,25]$. By further optimizing and uniting these factors towards multi-component approaches, attempts are made for the bioengineering of functional and viable bone constructs.

Although these described strategies provide options for BTE, realization of viable bone constructs for the treatment critical size bone defects remains a challenge. Keeping such a large bioengineered construct alive in the lab (in vitro) and after implantation in vivo requires sufficient transport and diffusion of oxygen and nutrients. In mammalian tissues, optimal diffusion efficiency is guaranteed by the natural organization and position of blood vessel capillaries, which are often located no more than 100-200 $\mu \mathrm{m}$ away from cells in a tissue [27-29] and holds the same for mature osteons and trabecular bone (100 - $150 \mu \mathrm{m}$ ) [30]. Although blood vessels do grow into engineered tissue constructs after they have been implanted in vivo (extrinsic vascularization), the ingrowth rate is low and not sufficient to supply the cells inside the construct with blood flow before they starve. Consequently, this causes a non-uniform cell survival, differentiation and poor integration $[28,31,32]$. This leads to the thought that bioengineered tissue constructs require an in vitro engineered vascular network to improve cell viability, and to facilitate a faster connection to the host circulation upon implantation in vivo.

\section{Vasculature}

The native vascular network is a hierarchical system in which several distinct structures can be distinguished, and is often described as a vascular tree. Listing them from large to small: the macrovessels (arteries and veins, $>100 \mu \mathrm{m} \varnothing$ ) branch off into microvessels (arterioles, metarterioles and venules, named 'mesovessels' $10-100 \mu \mathrm{m} \varnothing$ ) and finally transition into the smallest type of microvessels, the capillaries $(<10 \mu \mathrm{m} \varnothing)$ that diverge into the surrounding tissue [33-35] (Figure 1.1). During the gradual transition from macrovessel to capillaries, the inner diameter, thickness of the vessel wall and amount of layers of vascular smooth muscle cells (vSMCs) decreases, ending with a capillary structure that contains only a single layer of endothelial cells (ECs) and supporting pericytes. In line with the gradual decrease in diameter, the blood pressure and flow rate drop, which allows for the diffusion of nutrients, fluids and gasses into the tissue and waste removal from the tissue into the capillaries [36]. 




Figure 1.1. Hierarchy of the vascular network. The macrovessel (artery) branches off into an arteriole (mesovessel) that transitions into capillaries that provide the tissue cells with oxygen, tissue fluids and nutrients. The waste products are removed from the tissue and transported back via the venous system. The microvascular system also has an important angiocrine function and serves as a transportation system for circulating cells.

While the most recognized role of the microvascular system is the capillary to tissue fluid exchange, their angiocrine function should not be overlooked. Paracrine factors from the vessel sustain the homeostasis of tissue-specific stem cells in the vicinity of the blood vessel and guide the regeneration of damaged tissues [37]. Moreover, bone tissue requires a vascular system for the supply of minerals and as a source of circulating progenitor cells, plus it serves as a template around which bone development takes place [15, 37, 38]. In more detail, a synergistic coupling of angiogenesis and osteogenesis is present, in which the endothelial cells secrete BMP2 and BMP4 to induce osteoblast differentiation and to enhance osteoblastic function [37, 38]. The uptake of BMPs by the osteogenic cells induces Run-related transcription factor 2 (RUNX2) activity, resulting in the expression of proteins involved in osteogenesis (e.g., bone sialoprotein (BSP), osteocalcin, and osteonectin) [39]. In turn, the osteoblasts secrete angiogenic factors (e.g., PDGF-BB and VEGF) that support angiogenesis and vessel stabilization. VEGF additionally attracts monocytes/macrophages and acts on the recruitment of osteoclasts in a dose dependent manner [37]. This underlines the extensive cellular crosscommunication between the processes of osteogenesis and angiogenesis, which balances bone formation and resorption during skeletal development and bone repair.

\section{Regenerative strategies for vascular tissue engineering}

The importance of this osteo-vasculogenic synergy emphasizes the need for vasculature in $\mathrm{BTE}$, and serves as a basis for the design of many bone regenerative strategies. Although the blood vessels of the patient can naturally anastomose to an implanted in vitro engineered 
capillary network within several days, immediate perfusion of the bioengineered tissue construct after implantation is unattainable since capillaries are too small for surgical anastomosis [27, 28]. Additionally, without a proper perfusion of the engineered pre-vascular network, maturation of the capillary-like network is incomplete and cannot fully lumenize or interconnect with the host's vessels (reviewed in [40]). This immature state of the network prohibits further perfusion to the center of the construct and can lead to improper integration.

To realize an effective and immediate connection of bioengineered constructs to the patient's circulation directly after implantation, it is suggested to follow the native architecture of the vascular network (i.e., larger vessels gradually transitioning into capillaries) and to incorporate this in the engineered tissue construct already in vitro. Developing a multi-scale in vitro engineered vascular network with these different levels of vasculature would allow for the surgical connection of macrovessels to the patient's blood supply after transplantation. This would then effectuate and instantaneous perfusion of the engineered capillary network of the tissue construct. Various strategies for the bioengineering of vessels are currently under investigation, with the specific design requirements tuned to the natural architecture and structural components of the vessel type. Several strategies to create the different levels of vasculature are specified below.

\section{In vitro macrovessel engineering}

The bioengineering of free-standing macrovessels, also known as tissue-engineered vascular grafts (TEVGs), has already received a considerable amount of attention. TEVGs can be designed for different purposes, such as serving as stand-alone motilities for vessel replacements, as a shunt for hemodialysis procedures or for patients that are in need of a bypass [41]. Recent focus has been on the bioengineering of small diameter vascular grafts ( $<6 \mathrm{~mm}$ in diameter) due to problems of the currently used synthetic grafts (e.g., Gore-Tex or Dacron) related to intimal hyperplasia, thrombosis and consequently stenosis [42-46]. Approaches for creating vascular grafts can be based on natural or synthetic scaffolds, soft hydrogels, or composites thereof [41-43]. Engineering of such grafts involves commonly used methods like decellularization of native materials, molding, rolling of cell sheets and direct scaffolding (e.g., electrospinning) [41, 47-49] (Figure 1.2). Several approaches already moved to clinical applications and have been implanted in patients, for example cell-based sheets (Cytograft [50] and LifeLine [51, 52]), biodegradable constructs [53] and decellularized grafts (Synergraft [54], Artegraft [55] and Humacyte [56]). Despite their promising performance in terms of patency and patient survival, these approaches often rely on time-consuming manual fabrication and still show high prevalence of graft rejection and stenosis. This indicates that work remains to be done to improve their overall efficacy. As a possible solution, direct scaffolding methods like electrospinning, as investigated in this thesis, offer the possibility to improve the mimicry of the native vascular morphology in the constructs, as they have more control over the production process compared to the other methods [57-59]. 
When aiming to improve the mimicry of the native vessel, it is important that the construct's structure and physiological function resembles those of the native blood vessel, including low thrombogenicity, vasoactive properties and remodeling responses [60]. These tasks are normally carried out by the different tissue layers of the vessel, where each of the layers has an integral role in the overall function of the vessel [49] (Figure 1.2). Recapitulating the native cellular and extracellular structural organization (hierarchical mimicry), including these tissue layers, is therefore considered an important factor when attempting to simulate the vessel's properties. In general, natural blood vessels are comprised of a luminal layer (tunica intima) containing an anti-thrombogenic monolayer of ECs attached to the basement membrane. A secondary medial layer (tunica media) comprises circumferentially orientated vSMCs, necessary for the vasoconstriction, dilatation and elasticity of the vessel. The tunica media is surrounded by a layer composed of collagens and fibroblasts, which provides the blood vessel with supportive strength (tunica adventitia) [58]. Simulating the layered structure of macroscale vessels has gained interest in the field of regenerative medicine and several multi-layered scaffolds have been designed with advanced properties, compared to single layer constructs [58, 61-64]. Besides the hierarchical mimicry of the scaffold design, the graft should also include the correct mechanical properties such as sufficient burst strength and compliance $[41,42,60,65]$.



Figure 1.2. Strategies for in vitro macrovessel engineering. Commonly used approaches for tissueengineering of vascular grafts (TEVGs) include decellularization of native vessels, molding of hydrogels, rolling of cell sheets and direct scaffolding (e.g., electrospinning). An example of how TEVGs can be fabricated to resemble the layered structure of the native blood vessel, i.e., the luminal layer (tunica intima) containing a monolayer of ECs attached to the basement membrane, the tunica media comprising circumferentially orientated vascular smooth muscle cells, and the tunica adventitia with longitudinally aligned collagens and fibroblasts. 


\section{In vitro microvessel (capillary) engineering}

On the other end of the spectrum of the vascular tree, ample research has been dedicated to the engineering of the smallest type of microvessels: the capillaries $(\sim 10$ $\mu \mathrm{m}$ in diameter). These consist of a single endothelial layer that is enveloped by pericytes, with which ECs share their basement membrane [66-68]. A large body of work has been devoted to recapitulate the microvasculature in vitro in the shape of microvascular beds or micro-channels within a bulk material (Figure 1.3). Commonly, the material used for the bulk are hydrogels; a material which provides an environment that shares many features with the natural ECM, including high water content and viscoelastic properties with tunable stiffness [69]. Hydrogels can be produced from natural materials (collagen, fibrin and Matrigel), synthetic materials (polyethylene glycol (PEG)) or a combination thereof (gelMA, gelatin-PEG or acrylated hyaluronic acid with RGD sites) (reviewed in $[41,69,70]$ ). These hydrogels can be assembled into prescribed geometry via a variety of approaches, such as 3D bioprinting, laser degradation, or volumetric bioprinting. On the other hand, a microvascular bed can also be created via the self-assembly of the selected cell sources within the bulk material $[41,71]$ (Figure 1.3).
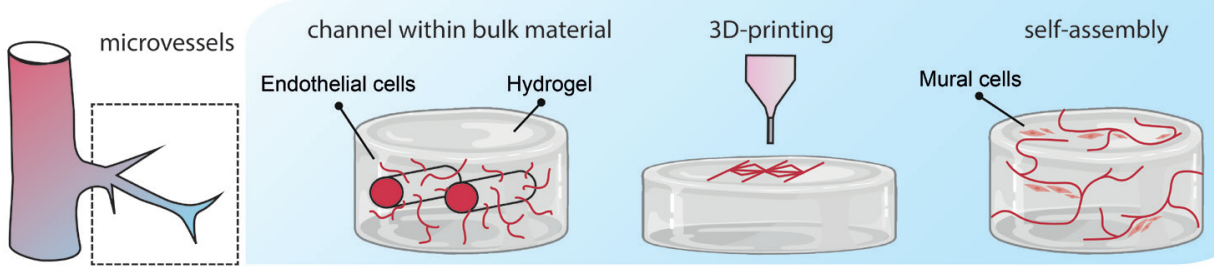

Figure 1.3. Strategies for in vitro microvessel engineering. Commonly used approaches for the engineering of a pre-vascular network include the fabrication of endothelialized channels within bulk materials (e.g., hydrogels), 3D printing of vascular structures or formation of a capillary-like bed via self-assembly of endothelial cells with mural cells.

For the engineering of a vascular bed it is critical to select a suitable cell source to achieve functionality, to support integration and to avoid immunologic reactions. It is also an important factor to take into account for clinical translation, when aiming at using cellbased constructs. A wide variety of cell populations has been explored, including tissuespecific ECs [72], human umbilical vein endothelial cells (HUVECs) [73, 74], endothelial progenitor cells (EPCs) [74-76] or ECs differentiated from (induced) pluripotent cells [77, 78]. Nonetheless, culturing solely ECs in a hydrogel construct will not lead to the desired vascular bed, as the survival and maturation of the vascular network is dependent on the support of cells from the perivascular lineage and would regress without their sustenance $[79,80]$. A feasible approach to overcome this problem is co-culturing ECs with a mural cell population, for which isolated pericytes, fibroblasts or progenitor-derived mural 
cells (e.g., from MSCs or induced pluripotent stem cells (iPSCs)) can be used [81]. When specifically focusing on pre-vascularization of osteogenic constructs, it is vital that (one of) the added cell types can differentiate into the osteogenic lineage with coinciding support of the vasculogenic process [82-86]. Osteogenic stimulation of such co-cultures can be achieved via the supply of an osteogenic differentiation medium (dexamethasone, $\beta$-glycerophosphate) [81], entrapment of osteogenic growth factors (TGF- $\beta$, BMPs), differentiation via CaP ceramics or supplements of magnesium or zinc (reviewed in [24, 87]). However, care should be taken to not disturb the cross-communicative balance between vasculogenesis and osteogenesis via the addition of such osteogenic factors, which on their turn might negatively affect vasculogenesis. Finding the optimal coculture conditions for all cell types involved remains a challenge inherent to all co-culture approaches.

\section{Interconnectivity of multi-level vasculature}

The challenge now is to merge the two levels of vasculature together in one construct. Here, the macroscale grafts could serve as a central vessel in pre-vascularized or nonvascularized tissue constructs, from which angiogenesis and subsequent interconnection could occur. This would mimic the in vivo situation described as the arteriovenous loop (AV-loop) model. Here, an autologous axial vessel serves as an intrinsic blood supply, from which capillaries sprout into the implanted construct, and is seen as an 'in vivo vascularization method' (Figure 1.4). In the AV-loop an artery and a vein are anastomosed into a loop shape and implemented in a chamber together with a carrier material, specified to the tissue of interest. Other approaches for in vivo vascularization with an intrinsic vascularization include, for example, the implantation of a porous scaffold material around a vascular bundle (e.g., femoral bundle) [88, 89]. In both cases, capillaries will sprout from the loop/bundle into the surrounding matrix, increasing the perfusion in defected areas and enabling the development of large vascularized constructs. In the case of bone constructs, the AV-loop chamber can be filled with an osteoinductive/ osteoconductive matrix (CaP ceramics) $[90,91]$ and provides a controlled environment for the delivery of osteogenic cells (e.g., osteoblasts or MSCs) $[92,93]$ and growth factors (BMP2, VEGF, FGF) $[93,94]$. Since the development of the AV-loop model, researchers have exploited it to regenerate bone defect in rat, rabbits, sheep and goats [90, 95-100]. The resulting constructs have proven to be functional, even after implantation in irradiated tissues [101]. Recently, the model was used for the first time to reconstruct small tibial and radial bone defects in human patients [102], which might showcase the potential of this system for BTE purposes. Yet, it still holds room for improvement and upscaling by eliminating the use of an autologous vessel and/or combining the carrier material with an in vitro engineered micro-vascular bed to accelerate their interconnection. 
Most research that focused on recapitulating the interconnectivity of the vascular tree in in vitro multi-scale vascular networks, has concentrated on the capillary sprouting from microchannels into bulk materials, or connection of pre-capillaries to a central channel [48, 103-109], rather than to/from free-standing vessels. Even though such microscale platforms provide detailed insight in the underlying mechanisms of sprouting, they do not yet offer the opportunity for a surgical anastomosis for tissue implantation and often bear low mechanical properties. Promoting sprouting from a TEVG would provide viable options to overcome this problem, since they are mechanically more stable and can undergo surgical anastomosis. However, the inherent design of most TEVGs is to support luminal endothelialization and to prevent the ingrowth of vSMCs (intimal hyperplasia). To avoid the latter, grafts are often fabricated containing a dense scaffold layer to avert cellular migration from the external side to the intima [110]. For the effective interconnection of newly formed capillaries to the vascular scaffold, and for endothelialization in case of in situ vessel regeneration, transmural capillaries should maintain the ability to penetrate through the (medial) scaffold layers. To allow for this transmural sprouting to happen, bigger sized pores should be integrated in the scaffold design [111]. With the increasing knowledge and technological progress in the field of biofabrication, scaffold designs could be realized that meet these requirements. This could expand the application of bioengineered macroscale vessels towards the generation of multi-scale vascularized bone tissue constructs (Figure 1.4).

A

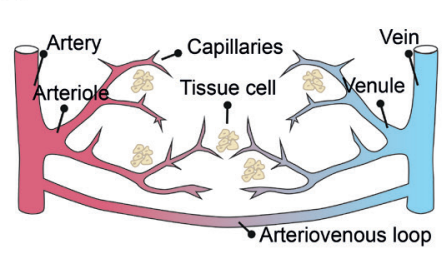

B


(n) 
the host vasculature, within an engineered bone tissue construct featuring a microvascular bed. To achieve this, we have developed strategies for the bioengineering of two levels of vasculature (macrovessels and microvessels), in which the microvasculature is combined with osteogenic cells for BTE purposes. This thesis is therefore divided into two parts; part I focusses on the bioengineering of macroscale vessels and part II shows the fabrication of microscale vasculature in osteogenic constructs.

Part I: The basis for the design of the macrovessel was a synthetic heterotypic vascular scaffold created by means of two electrospinning techniques, and is described in chapter 2. The scaffold was specifically designed to mimic the architecture of a native vessel, and was found to direct physiological tissue-like cell organization and differentiation of clinically relevant progenitor cell populations. To further mature the bi-layered graft into a functional vessel, luminal perfusion was imposed in a custom-made two-compartment bioreactor chamber. The compartments further enabled exposure of the graft's luminal side and the medial layer to tissue-specific media, as described in chapter 3.

Part II: In chapter 4 of the thesis, an osteogenically stimulated tissue including a microvascular bed was realized in a hydrogel model containing a co-culture. This set-up enabled the fabrication of a construct that served as an in vitro model, in which the influence of donor variation of clinically relevant cell sources could be assessed. In chapter 5 this coculture model was extended by the addition of CaP ceramics. These grafts were implanted subcutaneously in rats to determine their optimal in vitro osteogenic pre-differentiation period to induce in vivo bone formation. The outcomes of this chapter served as the basis for implantation in an autologous AV-loop model. This AV-loop model is described in detail in appendix 1 and provides background information on the literature and a study design of the envisioned in vivo follow-up study. At last, chapter $\mathbf{6}$ focused on the in vitro effects of an osteogenic stimulation on the microvascular bed in the co-culture model.

Finally, in appendix 2, parts I and II are combined and a method was suggested to connect the engineered macrovessel to the self-assembled microvascular bed in vitro. Possible adaptations to the heterotypic macrovessel scaffold design are described that may accommodate interconnection of the lumen with the outer compartment. Additionally, a technique is proposed to mold the microvascular co-culture model around the macrovessel to achieve an interconnected multi-scale vascular network, with the possibility for perfusion in the developed bioreactor system. 







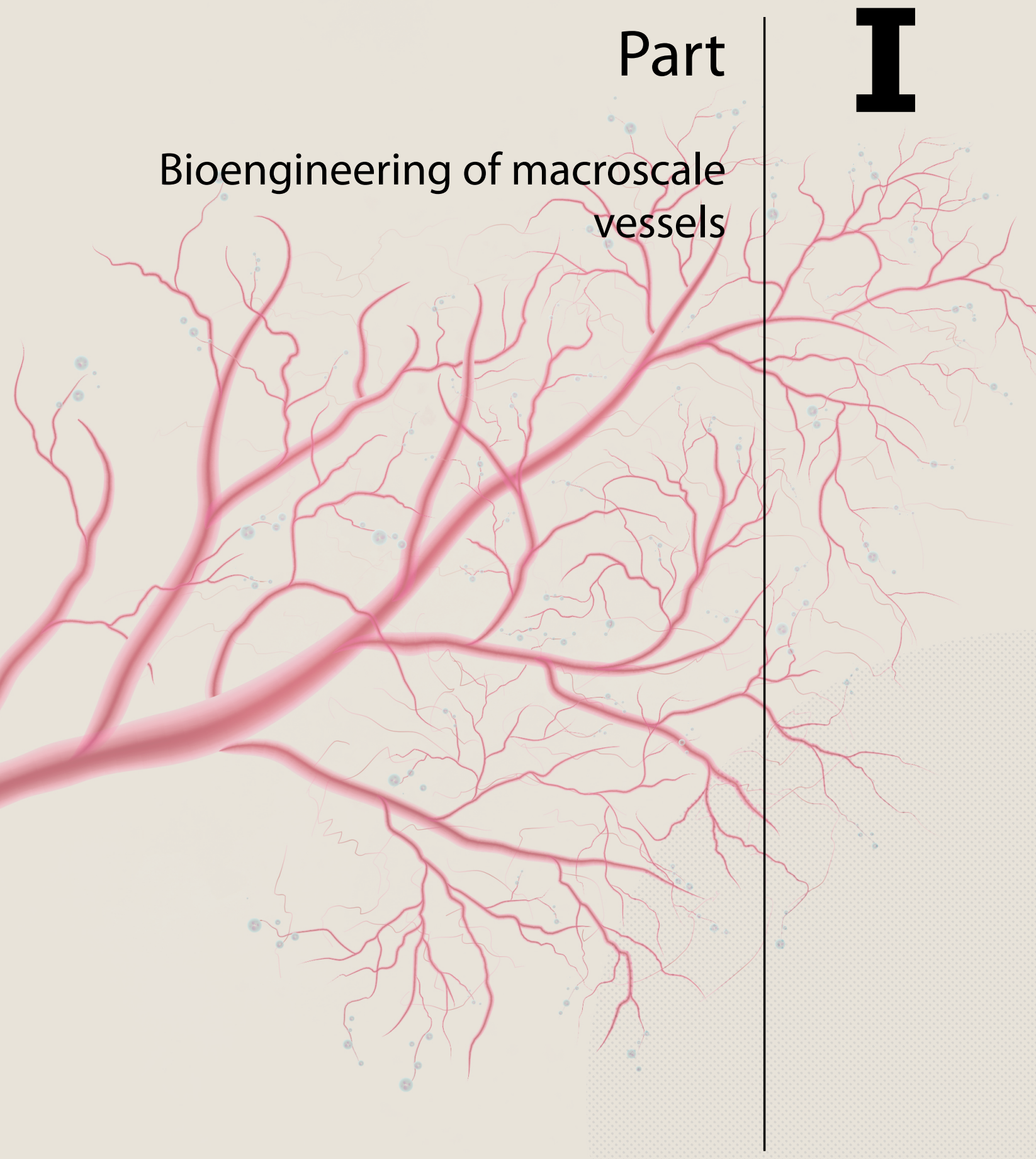



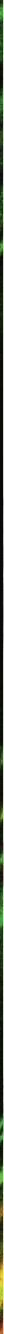

1

(1)
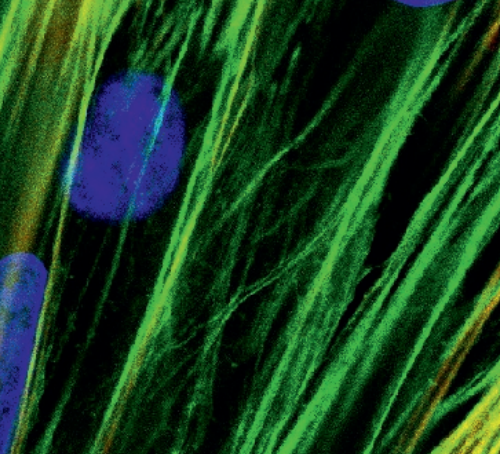


\section{Heterotypic scaffold design orchestrates primary cell organization and phenotypes in co-cultured small diameter vascular grafts}

this chapter is based on: Heterotypic scaffold design orchestrates primary cell organization and phenotypes in co-cultured small diameter vascular grafts. I. Pennings ${ }^{*}$, T. Jungst", M. Schmitz, A.J.W.P. Rosenberg, J. Groll\#, D. Gawlitta\# Advanced Functional Materials, 2019. 29(43).

*,\# these authors contributed equally 


\section{Abstract}

To facilitate true regeneration, a vascular graft should direct the evolution of a neovessel to obtain the function of a native vessel. For this, scaffolds have to permit the formation of an intraluminal endothelial cell monolayer, mimicking the tunica intima. As well, when attempting to mimic a tunica media-like outer layer, the stacking and orientation of vascular smooth muscle cell (vSMC) should be recapitulated. An integral scaffold design that facilitates this has so far remained a challenge.

We introduce a hybrid fabrication approach by combining solution electrospinning and melt electrowriting. This allows a tissue-structure mimetic, hierarchically bilayered tubular scaffold, comprising an inner layer of randomly oriented dense fiber mesh and an outer layer of microfibers with controlled orientation. The scaffold supports the organization of a continuous luminal endothelial monolayer and oriented layers of vSM-like cells in the media, thus facilitating control over specific and tissue-mimetic cellular differentiation and support of the phenotypic morphology in the respective layers. Neither soluble factors, nor a surface bioactivation of the scaffold is needed with our approach, demonstrating that heterotypic scaffold design can direct physiological tissue-like cell organization and differentiation.

\section{Graphical abstract}

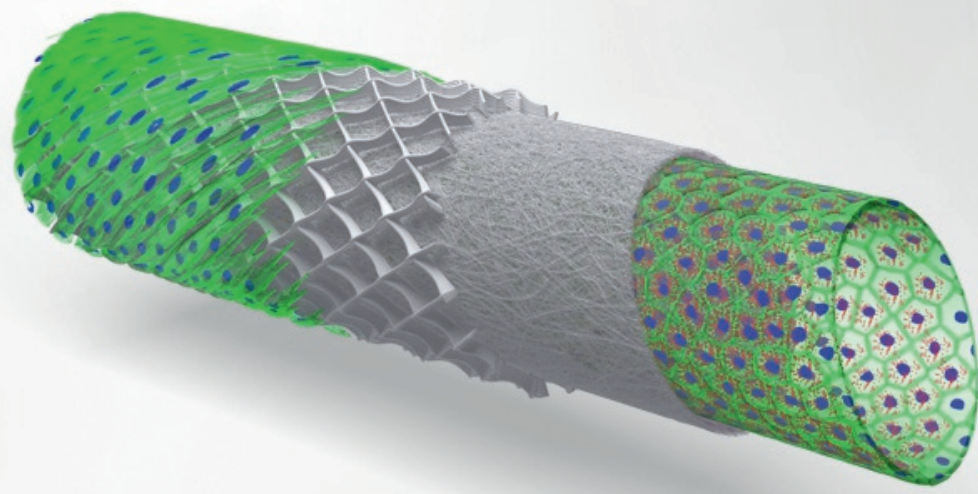




\section{Introduction}

Vascular grafts with improved long-term efficacy are a great clinical demand. Especially the replacement of small diameter blood vessels $(<6 \mathrm{~mm})$ remains a major challenge, due to problems associated with occlusion of the grafts, intimal hyperplasia or thrombosis $[43,58]$. The current clinical gold standard is to use autologous vessels, which is restricted by limited availability. Due to this and other limitations, biofabrication is a promising alternative approach for creating vascular grafts.

Natural blood vessels are, inter alia, comprised of a luminal layer (tunica intima) containing a single anti-thrombogenic monolayer of endothelial cells (ECs) attached to their basement membrane [58], and a secondary contractile medial layer containing nearcircumferentially oriented vascular smooth muscle cells (vSMCs) which are embedded in an independent basement membrane matrix (tunica media) [66]. Efforts for tissue engineering of vascular grafts are mostly focused on re-creating these two layers, and results in bi-layered scaffolds. Approaches for creating vascular grafts can rely on scaffolds, on soft hydrogels, or composites thereof $[42,112,113]$, and numerous studies have demonstrated promising results in the field of vascular tissue regeneration. Examples of studies that have shown promising in vivo performance include completely biological hydrogels, in the form of human cell-based sheets [114, 115], or biopolymer-based attempts in the form of hydrogels [116-120]. Despite the promising performance, those approaches rely on time-consuming and often manual fabrication. Here, scaffold-based approaches are of advantage, as they also offer the possibility to improve cell-material interactions and true hierarchical biomimicry of the native morphology in the constructs.

Among the applied fabrication methods, electrospinning of polymer solutions (solution electrospinning, SES), a process that predominantly yields nonwoven mats of fibers with diameters in a nanometer to micrometer range, has received considerable attention [5759]. The morphology of SES scaffolds can be adjusted as the fiber diameter influences the pore size of the nonwoven mats. Besides biochemical cues, the pore size is the main factor that promotes endothelialization of the luminal layer $[61,110,121]$. It was suggested that to achieve a monolayer of endothelial cells, pore sizes should not be greater than the size of a cell [61, 122]. Especially for small diameter vascular grafts, this monolayer is critical to avoid intimal hyperplasia and thrombosis.

Recently, a new fabrication technique called melt electrowriting (MEW) that employs controllable polymer melts instead of solutions has emerged [123-126]. Due to the viscoelastic properties of the polymer melt, the chaotic instabilities, which occur during SES, are suppressed. The stretched polymer jet can, in combination with an automated collector plate, be used for direct writing of structures [126, 127] composed of polymer fibers with diameters in the range of several hundreds of nanometers [128] to micrometers 
[129]. Compared to traditional additive manufacturing approaches like fused deposition modelling, the reduction in fiber diameter in combination with the control of deposition of the fibers enables the generation of constructs with a higher fiber density, a higher surface to volume ratio and a better control over pore architecture at a micrometer level. Using cylindrical targets and dedicated software, we have shown that it is possible with MEW to create tubular constructs with precise control over the fiber angle relative to the longitudinal axis of the tube [130].

Altering the fiber orientation is beneficial for the creation of biofabricated vascular grafts, as the orientation of the vSMCs and the extracellular matrix (ECM) in the tunica media is of importance for the contractile function of blood vessels [58, 62, 63, 112, 131, 132]. In the tunica media, collagen fibers predominately run in the circumferential direction but can also be oriented helically which is decisive for the circumferential mechanical properties of the vessel $[133,134]$. Recapitulating the regulation of cellular orientation in the tunica media of biofabricated vascular grafts has already been the point of focus in several studies, but was performed with limited control over fiber density in the selected fabrication techniques [62,63, 135-137]. This often resulted in medial layers with high fiber density, lacking in space for adequate cellular interactions and resulting in slow vSMC colonization $[136,138,139]$.

In the native situation, cells residing in the tunica media and tunica intima have extensive cross-communication. Therefore, a major advantage of tissue mimetic bi-layered constructs is the introduction of co-cultures with associated cellular crosstalk. The adequate function of a blood vessel is based on an EC monolayer that can stimulate the vSMCs by specific signaling pathways, such as the Alk1/Alk5/TGF $\beta$ pathway for steering the plastic phenotype of the vSMCs, or the secretion of nitric oxide (NO) via endothelial nitric oxide synthase (eNOS) for vasoconstriction and dilatation of the blood vessel [140, 141]. Despite its relevance, the interplay between the cell types in an engineered blood vessel are rarely addressed. So far, most studies only have reported bi-layered electrospun scaffolds supporting the culture of endothelial cells and vSM-like cells separately [142144]. Only few have shown simultaneous culturing of these cell types on bi-layered tubular scaffolds in vitro [61, 136, 145-147], but fall short in extensive phenotypical characterization of the cell layers. Moreover, none have taken relevant cell sources into account for the perspective of an eventual clinical application. To integrate that in the experiments from the beginning, the use of autologous smooth muscle cells, in case of vascular grafts, would be the best option. Still, in vitro this has been reported as a challenge due to their limited availability and proliferative capacity, and their switch to the synthetic phenotype, which challenges clinical translation [148]. Therefore, multipotent mesenchymal stromal cells (MSCs), which have the ability to differentiate into vSMCs, are an appropriate alternative for vSMCs [149-153]. Further, as a source for endothelial cells, endothelial progenitor cells 
(EPCs) can be used, which can also be harvested from an easily obtainable cell source (e.g., human umbilical cord blood and peripheral blood). Among the EPCS, a subgroup named "endothelial colony forming cells" (ECFCs) show high expansion potential and inherent vasculogenic and angiogenic capacity [154, 155]. Both MSCs and ECFCs can originate from autologous sources and are thus suitable for clinical translation of biofabricated vascular grafts.

Following an analysis of the architecture of a native human artery, the underlying hypothesis of this study was that, if an advanced scaffold can be fabricated with tissuemimetic layered hierarchy combined with a heterotypic topology, this scaffold can direct the formation of vessel-like cell organization, orientation and differentiation upon seeding of ECFCs and MSCs onto the respective layers. Heterotypic topology means a basalmembrane like morphology at the inner lumen for endothelial cells, and an adhesion and migration guidance for vSMCs or their progenitors in the outer layer in a tissue-analogous orientation towards the circumferential axis of the constructs. We further hypothesized that such scaffolds may result in tissue-analogous cell organization and phenotype evolution without the need for additional soluble factors or a surface bioactivation of the scaffolds.

\section{Results and Discussion}

Tubular scaffolds, as a basis for the biofabricated vascular grafts, were thus fabricated by combining SES and MEW of poly( $\varepsilon$-caprolactone) (PCL) through consecutive fiber deposition (first SES, then MEW) onto a cylindrical target with an outer diameter of $3 \mathrm{~mm}$. These scaffolds were then seeded with ECFCs and MSCs. The SES nonwoven inner layer enabled the ECFCs to organize into a continuous endothelium. Furthermore, the ECFCs expressed signals associated with cross-communication towards vSMCs. The MEW layer controlled the orientation of the MSCs, was fully populated with cells, facilitated close cell-cell contacts, and accelerated the differentiation of MSCs into vSM-like cells. Along, it was shown that the scaffold could support both cell types when co-cultured, providing a platform in which cellular cross-communication can be studied.

In order to know how to design biomimetic bi-layered scaffolds with heterotypic topology, first the architecture and phenotypic aspects of a native human muscular artery were established by analyzing the presence of a selection of contractile vSMC markers and associated ECM components (Supplementary Figure 2.1). Calponin, a-smooth muscle actin (a-SMA), smooth muscle myosin heavy chain (SMMHC), laminin a5 and collagen type IV were identified and located in the natural vessel tissue (Supplementary Figure 2.1 B-F). Also, overall tissue hierarchy was established (H\&E staining, Supplementary Figure 2.1 A). The tissue layers could be distinguished clearly, with the relatively thin tunica intima (10-20 $\mu \mathrm{m})$ on the luminal side of the vessel with its collagen type IV-positive basement 
membrane and a fine network of connective tissue with elastic fibers. The native tissue stained positive throughout the whole thickness of the tunica media ( 400 $\mu \mathrm{m})$ for the contractile vSMC and ECM markers of interest. Additionally, the vSMCs were organized in an elongated, concentric, and multi-layered manner.

Based on the analysis of the human blood vessel and on the literature search presented in the introduction, design criteria for the biofabricated vascular grafts-scaffolds were formulated. The newly designed bi-layered small diameter vascular grafts $(3 \mathrm{~mm} \varnothing)$ should provide both the ECFCs and the MSCs with the appropriate environment, mimicking and instructing the cells to organize and deposit matrix in the native architecture. To achieve this, we defined the following key consideration points:

1. The scaffold should provide a substrate on which the ECFCs form a continuous monolayer.

2. The endothelial monolayer should express mature EC markers, components of the basement membrane and ECM, and signals associated with crosscommunication towards vSMCs.

3. The scaffold should provide a porous outer layer that vSMCs can migrate in and fill to achieve a multi-layered elongated cell organization.

4. The vSMCs should align in a near-circumferential orientation, specifically controlled by the MEW fiber orientation.

5. The scaffold should provide an environment for the MSCs to differentiate into the vSMCs contractile phenotype.

As these design criteria could not be met by any single fabrication technique, we developed a new fabrication procedure by combining two techniques, solution electrospinning (SES) and melt electrowriting (MEW) (Figure 2.1 A-B). This enabled the fabrication of scaffolds with so far unachievable biomimetic and heterotypic structural features as shown in Figure $2.1 \mathrm{C}$. The scaffolds were composed of an inner cylindrical nonwoven layer $(80 \pm$ $5 \%$ porosity, inner diameter $3 \mathrm{~mm}$ ) of solution electrospun poly( $\varepsilon$-caprolactone) $(\mathrm{PCL})$ fibers with a diameter of $1.4 \pm 0.2 \mu \mathrm{m}$ and a random orientation. The same material was used to create a layer of MEW fibers $(15.2 \pm 4.8 \mu \mathrm{m})$ with a winding angle between $30^{\circ}$ and $70^{\circ}$ and controllable large open pores (Supplementary Figure 2.2 and 2.3). As the material deposited onto the SES nonwoven by MEW was still at a temperature above its melt point, it could fuse with the fibers of the inner nonwoven as shown in Figure $2.1 \mathrm{C}$. This is crucial to avoid delamination of the layers during cell culture and when removing the samples from the cylindrical collector they are deposited onto. As revealed via balloon inflation burst experiments, the burst pressures of the (cell-free) constructs was $2400 \pm 75 \mathrm{mmHg}$. This value exceeded the required pressure for transition to clinical translation based on measurements of the saphenous vein of $1700 \mathrm{mmHg}$ [60] (Supplementary Figure 2.4). 

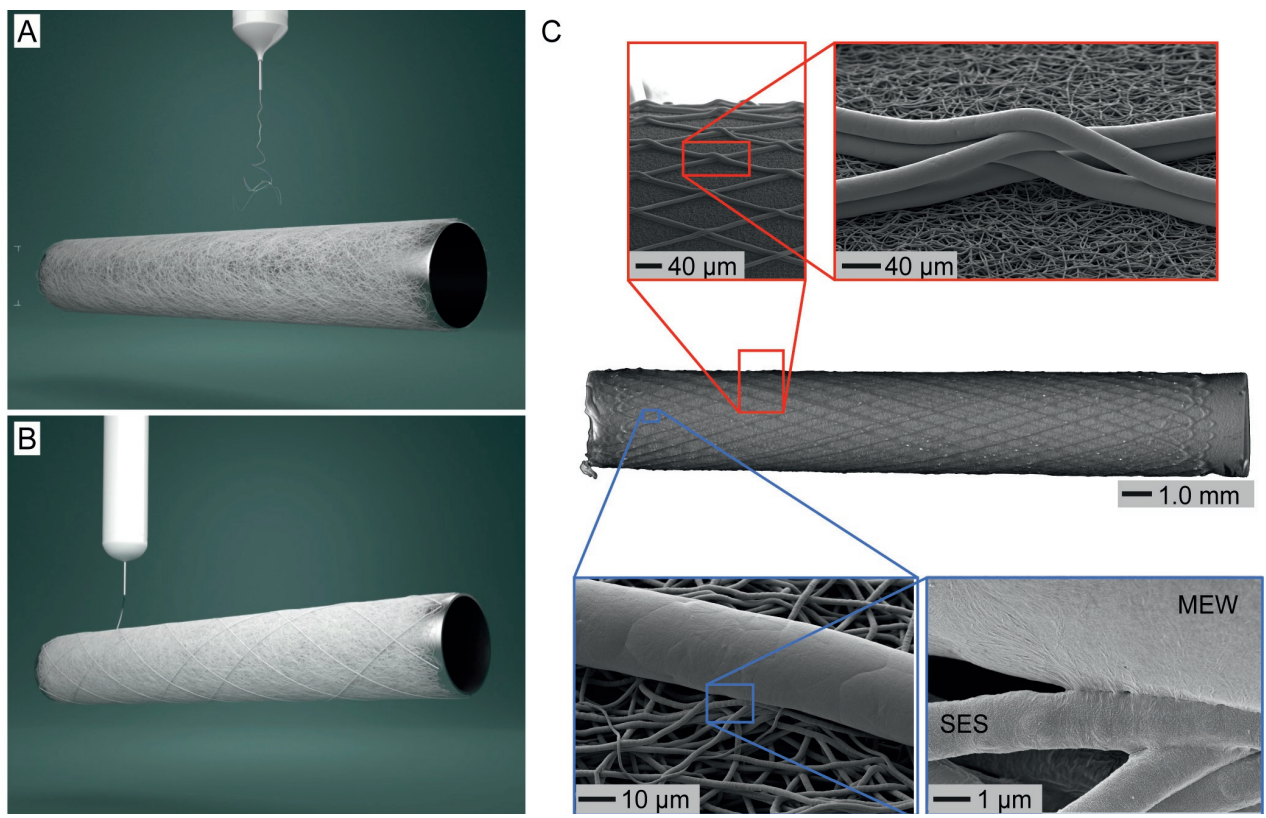

Figure 2.1. Preparation of bi-layered tubular scaffolds. A) Solution electrospinning is used to generate a tubular nonwoven inner layer. B) The rod with the nonwoven is transferred to a melt electrowriting device and oriented fibers are deposited on top of the nonwoven luminal layer. C) The final construct is removed from the cylindrical collector. Bi-layered scaffolds are made from one material and the fibers with different dimensions fuse, which prevents delamination.

The heterotypic electrospinning approach supported endothelialization on the inside of the dense luminal nonwoven layer, as illustrated by the presence of a confluent endothelial monolayer in scaffolds mono-seeded with ECFCs (Supplementary Figure 2.5 and 2.6 A-C). Importantly, this advanced scaffold design also supported so far unreached endothelialization in co-culture conditions of ECFCs with MSCs (Figure 2.2 A) and showed no infiltration of the ECFCs into the SES layer (Figure 2.2 A). The formation of a continuous monolayer meets the achievement of key point 1. Scanning electron microscopy (SEM) demonstrated the establishment of connections between neighboring ECs (black arrows) with their extrusions (white arrows), indicative of a restrictive endothelial barrier and low permeability (Figure $2.2 \mathrm{~B}-\mathrm{C}$ ). The low permeability and functional integrity of the monolayer was mostly supported by the specific redistribution of the mature endothelial cell markers CD31 and VE-cadherin towards the cell periphery (Figure 2.2 D-E), as validated previously $[156,157]$. The tight connections between the ECs via these interactions are a prerequisite for an endothelium to form a semi-permeable barrier, and are essential for their interaction in signaling pathways regarding endothelial plasticity, vascular integrity as well as sensing mechanical tensions, such as shear stress and to inhibit the activation of platelets and leukocytes $[157,158]$. Reproducing the functional integrity of theendothelium 
with barrier function is essential to resist thrombosis following introduction in vivo, and is thus required to be present in biofabricated vascular grafts to pass functionality and for long term patency [60]. The capability of the generated mono-cultured endothelium to prevent platelet adhesion was shown, while platelets did aggregate on the exposed scaffold surface (Supplementary Figure 2.6 D-F). With this, a lining with anti-coagulative properties was produced, resembling the properties of a native endothelium. Likewise, the monolayer showed positive endothelial marker staining for the platelet adhesion glycoprotein von Willebrand factor (vwF) (Figure 2.2 D) and was supported by a collagen type IV-positive matrix (Figure 2.2 E), as also found in the native basement membrane [66, 159], which supports the biomimetic properties of the scaffold. Expression of these ECrelated markers and ECM/basement membrane components were also confirmed on a gene expression level, both in mono and co-cultures (Supplementary Figure 2.7).

To meet the second key consideration point, signaling pathways for vSMC-EC communication were examined via assessment of nitric oxide (NO) secretion into the culture medium, and via gene expression of the Alk1/Alk5/TGF $\beta$ pathway for vSMC differentiation in co-cultured biofabricated vascular grafts. For MSCs of all 3 donors, combined with ECFCs, the product NO was detected in the medium (Figure $2.2 \mathrm{~F}$ ). Also, the presence of mRNA of the Alk1/Alk5/TGF $\beta$ markers was confirmed after coculturing (Supplementary Figure 2.7). This indicated the presence of the cellular crosscommunication for vasoactivity, which is required for the development of a functional endothelium in biofabricated vascular grafts [60]. Especially the production of NO, which is considered one of the predominant vasodilators and is involved in inhibition of platelet aggregation, is necessary for a functional endothelium [160]. The measured synthesis of NO in our culture system gives indications that the formed endothelium holds functionality and possesses the ability to signal to vSM-like cells when cultured on our biomimetic bi-layered scaffolds. Altogether, the expression of the markers for mature ECs, for components of the basement membrane and ECM markers, together with the indicated presence of the cellular cross-communication, shows that also the second key consideration point was met. 


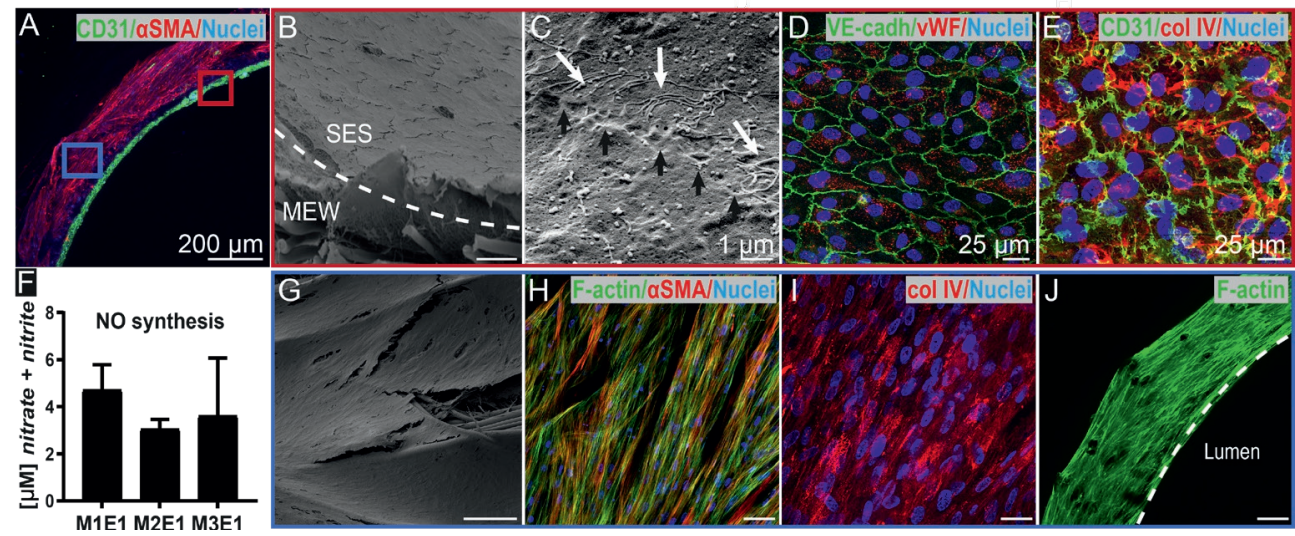

Figure 2.2. Cell culture on bi-layered heterotypic vascular grafts. A) Layered organization and distinctive phenotypes of simultaneously cultured ECFCs $\left(C D 31^{+}\right)$and vSM-like cells $\left(\alpha S M A^{+}\right)$after 17 days (cross-sectional view). B) Endothelialization of the SES layer on the luminal side with C) tight cell-cell connections (black arrows) and cell extrusions (white arrows), D) positive staining for the endothelial cell markers von Willebrand factor and VE-cadherin, the latter of which was located at the cell periphery, and E) CD31; also, a collagen type IV-positive basement membrane-like matrix was detected. F) The endothelial cells produced nitric oxide (NO) for signaling to the vSM-like cells (MSC donors $n=3, M 1-M 3$ ). G) vSM-like cells covered the medial layer in an aligned fashion, $H$ ) with elongated $\alpha \mathrm{SMA}^{+}$cells following the MEW fibers with I) a collagen type IV deposition in the direction of the cell alignment. J) vSM-like cells filled the whole thickness of the MEW layer and showed a circumferential orientation in mono-culture after 7 days. Scale bars represent $100 \mu \mathrm{m}$ unless stated otherwise.

Further, we demonstrated that the large pores and MEW fiber alignment could induce a fast infiltration of the vSM-like cells, to meet the third key consideration point, in both MSC/ECFC co-cultures (Figure $2.2 \mathrm{~J}$ ) and MSC mono-cultures (Supplementary Figure 2.8). The seeded MSCs covered the whole outer surface of the scaffold (Figure $2.2 \mathrm{G}$ ) and appeared in an aligned fashion (Figure $2.2 \mathrm{H}$ ). Moreover, the initially seeded MSCs showed the desired arrangement, indicated by the elongated cytoskeletons and aSMA-positive structures. Also, a collagen type IV was synthesized (Figure $2.2 \mathrm{I}$ ), which was enclosing the individual vSMCs as a basement membrane-like matrix (Supplementary Figure 2.9), mimicking the native situation $[66,159,161]$. To prove that the high control over MEW fiber placement could also guide the orientation of the MSCs, they were mono-seeded ( $n=4$ MSC donors) on scaffolds i) without MEW layer, ii) a MEW layer with fiber winding angle of $30^{\circ}$ (programmed angle $30^{\circ}$, measured angle $35.3 \pm 0.8^{\circ}$ ) and iii) samples with MEW layer at an angle of $70^{\circ}$ (programmed angle $70^{\circ}$, measured angle $71.6 \pm 0.6^{\circ}$ ). The cellular orientation in the MEW layer was analyzed based on the pixel orientation of the fluorescentlystainedF-actinfibers, throughout the wholethickness of the MEWlayer (Figure 2.3 and Supplementary Figure 2.10, detailed description in Supporting information). Cells seeded on the nonwoven mesh (i) were mainly oriented in the longitudinal direction of the tubular scaffold $\left(2.3 \pm 1.4^{\circ}\right)$. With increasing winding angles of the scaffold fibers, the 
cellular orientation changed into a near-circumferential direction (Figure $2.3 \mathrm{~A}$ ) as present in the native tunica media. The cells seeded on the constructs with a $70^{\circ}$ winding angle of the MEW fibers were oriented in an angle that was larger $\left(77.7 \pm 3.4^{\circ}\right)$ than the angle of the fibers. This winding angle was used in constructs for further cell culture experiments, as this met the fourth key consideration point of (near) circumferentially arranged cells. Moreover, this specific winding angle mimicked the helically orientated collagen fibrils as in the native arteries [131, 133], and steered the orientation of the vSM-like cells as in the human aortic tunica media $[162,163]$. Taken together, we thereby proof and verify our hypothesis that the orientation of vSMCs on tubular scaffolds can be guided in a tissue-mimetic manner by controlled orientation of fibers in the micrometer range. The open porous structure of the outer layer facilitated a fast cellular ingrowth and resulted in several layers of orientated aligned cells with close cellular interactions. We could achieve different cell orientations and found one that is potentially exploitable for facilitating vasodilation and constriction for next generation bioengineered vascular grafts.

A

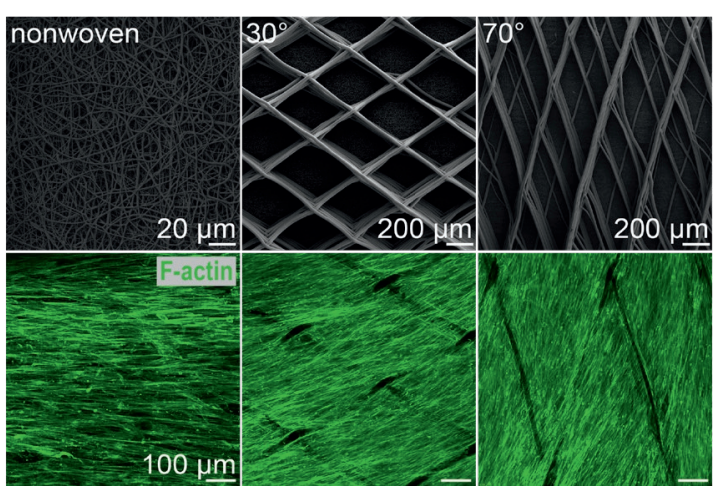

B

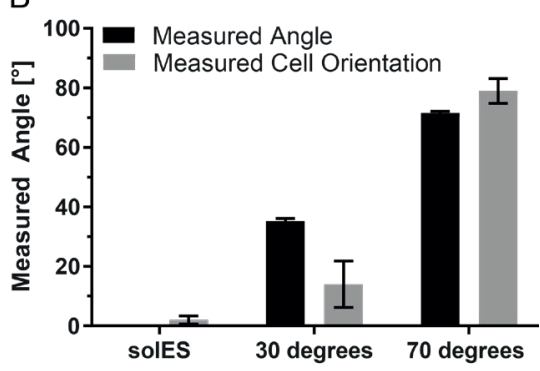

Figure 2.3. Influence of the orientation of melt electrowritten fibers on the orientation of MSCs. A) Representative scanning electron microscopy images of the scaffolds before seeding (top row) and a projection of F-actin stained cells from a 3D stack of scaffolds after seeding and culture for 7 days (bottom row). B) The 3D projection was used to analyze the mean cell orientation throughout the thickness of the MEW layer, and showed the average orientation of the melt electrowritten fibers as well.

The last key consideration point for aiming at engineering of the tunica media, is to realize the SMC phenotype switch to the contractile phenotype at the proper stage during their maturation process on the scaffold. Generally, differentiation of MSCs into contractile vSMCs is accomplished by the addition of biochemical factors associated with differentiation, such as transforming growth factor $\beta 1$ (TGF- $\beta 1$ ) or platelet-derived growth factor subunit $\beta$ (PDGF- $\beta$ ) $[152,161,164,165]$. Interestingly, we observed that by expanding bone marrow-derived MSCs in a culture plate, supplemented with the proliferation-associated basic fibroblast growth factor (bFGF), also an induction of differentiation was observed 
after reaching confluency. This differentiation was accompanied by protein upregulation of the contractile VSMC marker proteins aSMA and calponin, as well as elevated gene expression levels of additional contractile vSMC markers (Supplementary Figure 2.11). In addition, the cells showed capacity for contraction of a collagen lattice (Supplementary Figure 2.14). The induction of differentiation in post-confluent vSMC cultures has been reported previously [166] and several groups showed very promising results and could, for example, use the fiber orientation to control the 2D orientation of a monolayer of vSMCs $[167,168]$. It could be shown that the orientation, alignment and confluency can be utilized to control the phenotype of vSMCs $[169,170]$. The effect of confluency was also reported to be existent in MSC cultures by Alimperti et al in 2014, where the induction of differentiation of MSCs into contractile vSM-like cells was described, without the use of above-mentioned differentiation growth factors [171]. They hypothesized that the observed differentiation is a cell-cell junction-mediated process through the adherens junction cadherin-11, normally found on MSCs, with an associated autocrine action of TGF- $\beta 1$ [171], or possibly by the secretion of a basement membrane-like matrix associated with the contractile vSMC phenotype [132]. In addition, the MSCs used in our experimental set-up appeared to be positive for CD146 (Supplementary Figure 2.12), a marker previously associated with perivascular cells $[172,173]$, and were therefore more prone to vSMC differentiation.

To more closely investigate the effect of increased cell-cell interactions on the differentiation of MSCs in our scaffold with open pores, we compared their differentiation on constructs without or with MEW layer, as the latter enhances stacking of MSCs and thereby increases cell-cell contacts in a 3D setting. To do so, we first showed that the SES layer was a sufficient substrate for the MSCs to proliferate and differentiate on, to reach the same confluency as in a culture plate, with similar expression of contractile vSMC markers on both gene and protein levels (ACTA2 - aSMA; CNN1 - Calponin; Transgelin - SM22a and the extracellular matrix markers LAMA4 - Laminin subunit a4 and ELN - Elastin) after both 7 and 14 days (Figure 2.4 A, no significant differences). In this setup, the MSCs were mono-cultured on the single-layered SES scaffold without the MEW fibers and compared to the differentiation status of MSCs cultured in plates (control group). Accordingly, the SES proved to be a suitable substrate for culturing and differentiation (with longitudinal alignment) of MSCs into vSM-like cells, in comparable rates as on a culture plate.

Next, we analyzed the influence of MEW fibers on the differentiation process of MSCs into vSM-like cells. Therefore, MSCs were mono-cultured and a comparison was made between the single-layered scaffold (SES only, control group) and the bi-layered scaffold with the MEW fibers. After 7 days of culture, the cells on the bi-layered scaffolds had higher gene expression levels of the contractile phenotype markers aSMA (6.5 \pm 2.9$)$ and calponin (3.4 \pm 1.4 ) compared to the expression of MSCs cultured on the SES only layer (Figure 2.4 B), 
though not statistically significant. The same trend could be seen on a protein level (Figure 2.4 E-F). Additionally, the bi-layered constructs contained stacked vSM-like cell sheets, composed of more aSMA ${ }^{+} /$Calponin ${ }^{+}$cells compared to the SES only layer (Figure 2.4 E-F and zoom-in in Supplementary Figure 2.13). This proves the benefit of our biomimetic scaffold design as the MEW layer provides an adjustable tool to control the phenotype of the vSM-like cells.

A

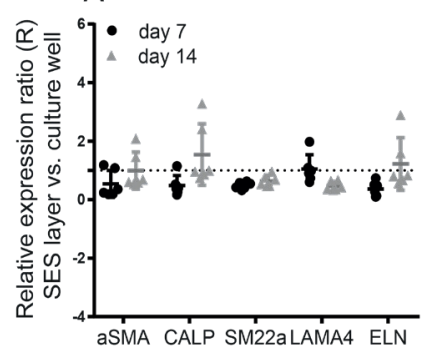
$\mathrm{F}$
$\mathrm{B}$
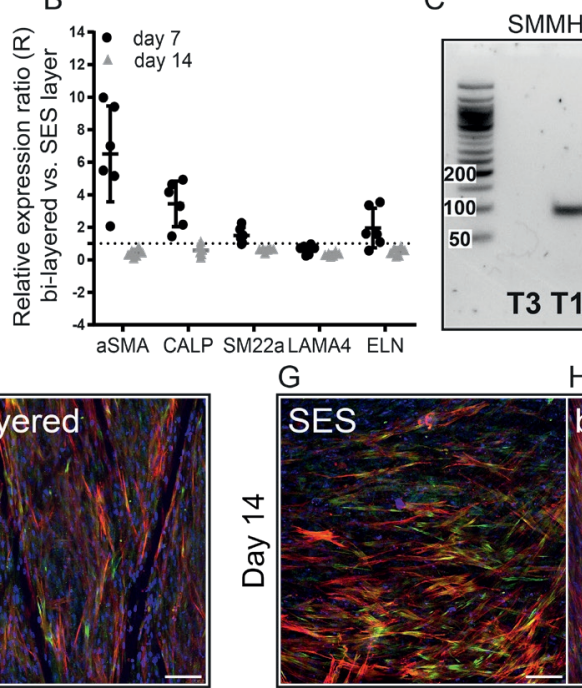

$\mathrm{D}$

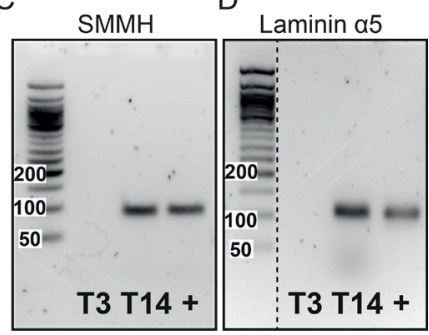

$\mathrm{H}$

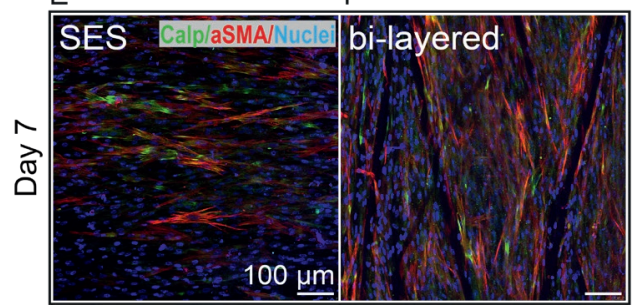

bi-layered

Figure 2.4. Confluence-driven MSC differentiation towards vSMCs. A) Similar relative gene expression levels of contractile vSMC markers of MSCs cultured on the SES only layer, compared to MSCs cultured in well plates after 7 and 14 days of culture, normalized to GAPDH. B) Comparison of gene expression levels of MSCs cultured on bi-layered vascular constructs versus MSCs cultured on the SES only layer after 7 and 14 days of culture, normalized to GAPDH. C) qPCR product on gel electrophoresis of SMMHC, representative for both SES-only and bi-layered scaffold cultures and D) laminin subunit a5; both $C$ ) and D) show qPCR products after 3 and 14 days of culture, compared to commercially obtained mature vSMCs (positive control). E) A small amount of aSMA ${ }^{+} /$Calponin ${ }^{+}$cells on SES only scaffolds in a confluent layer after 7 days, aligned with the $0^{\circ}$ axis, and $F$ ) in a circumferential multilayered oriented sheet on the bi-layered scaffold, also with aSMA ${ }^{+} / C_{\text {Calponin }}{ }^{+}$cells. G) Multi-layered vSM-like cells on SES only scaffold with increased numbers of aSMA ${ }^{+} /$Calponin ${ }^{+}$cells, compared to day 7 , and $\mathrm{H}$ ) highly organized $\mathrm{aSMA}^{+} / \mathrm{Calponin}^{+}$vSM-like cells on the bi-layered scaffold with elongated morphology after 14 days.

The differences in gene expression between the bi-layered scaffold and the single SES layer was not present anymore after 14 days. This could be explained by the slower proliferation of vSM-like cells on the SES only layer (Figure 2.4 B) between days 7 and 14, meaning that the induction of confluency-induced differentiation has a delay compared to the bilayered seeded scaffold. This was confirmed by the rapid establishment of 3D stacked cell sheets, and associated differentiation of the MSCs in the bi-layered graft by day 7 already 
(Figure 2.4 E-F). At both the early and late time points, comparable levels of relative gene expression of SM22a (1.5 \pm 0.5$)$, tropo(elastin) $(2.0 \pm 1.2)$, and LAMA4 (0.6 \pm 0.3$)$ were found in both groups. Overall, more $\mathrm{aSMA}^{+} /$Calponin ${ }^{+}$cells were present in the bi-layered scaffolds compared to the SES only scaffold. Thus, in addition to the previously shown beneficial effect of the MEW layer on the vSM-like orientation, the MEW fibers also seem to provide a substrate for the cells to attach to and proliferate faster into a 3D differentiated stack of cells. This in comparison to the, in general single layer of, vSM-like cells on the SES scaffold. Importantly, all vSMC markers were also present in the co-cultured constructs (Supplementary Figure 2.7). Taken together, this data evidences that our tissue-mimetic scaffold design with heterotypic morphology, achieved through conversion of SES and MEW onto cylindrical targets, provided an open-porous 3D microenvironment for the MSCs that induced differentiation into the contractile phenotype, proving that the fifth and final key point was met.

To evaluate if the MSCs indeed acquired an actual vSMC phenotype, we included a broad pool of markers related to the contractile SMC phenotype (double positive aSMA ${ }^{+}$/ calponin $^{+}$cells and SM22a), together with relevant ECM components (collagen type IV, laminin subunit a4 and (tropo)elastin). Noteworthy, analysis of only expression of calponin or aSMA as smooth muscle cell markers may fail to indicate the true differentiation state of MSCs, as non-differentiated MSCs and myofibroblasts have the capability to simultaneously express some of the vSMC contractile phenotype markers [174]. As such, in this study, the detection of aSMA/calponin-double positive cells was assessed, since these are most likely representing true vSMC cells, as previously reported by Liu et al., 2013. To further explore the true differentiation state of the MSCs towards vSM-like cells, two markers associated with the highest degree of vSMC differentiation specificity were selected to additionally confirm their fate by PCR. These were SMMHC, considered to be the most selective vSMC marker [148] and laminin a5 (laminin-511 and/or 521), normally found in the basement membrane of mature vSMCs [161]. MSCs cultured on the SES-only scaffold and bi-layered scaffold for 14 days (T14) expressed SMMHC, while this was absent after only 3 days (T3) of culture (Figure 2.4 C-D). Likewise, the vSM-like cells were found to express the laminin a5 subunit (Figure 2.4 C-D). In addition, the potential for contraction of the vSM-like cells differentiated on the scaffolds was confirmed in a collagen gel lattice contraction assay (Supplementary Figure 2.14). Hence, the expression of markers of the contractile vSMC phenotype in the biofabricated vascular grafts, their capacity for gel contraction, and in addition, the observations for the elongated morphology of the SMlike cells, are reliable indicators of a smooth muscle cell-like phenotype differentiated from MSCs. 


\section{Conclusion}

In conclusion, a new hybrid fabrication procedure was developed by combining two techniques, solution electrospinning and melt electrowriting. This allows for a tissuestructure guided fabrication of heterotypic bi-layered tubular scaffolds in a two-step approach with one order of magnitude difference in fiber thickness, within the same scaffold. The achieved tubular scaffold architecture resembled the dimensions and spatial organization of the intimal and medial layers of a native vessel. Thereby, the scaffolds were directing cell morphology and differentiation. The inner SES layer supported the formation of a confluent endothelium, with indications of a restrictive endothelial barrier function with low cellular permeability. The open-porous outer MEW layer with a low fiber density and controlled deposition and orientation was the fundament for a fast vSM-like cell colonization in an orientated 3D manner. The heterotypic scaffold design tackles, for the first time, the problems generally described with electrospinning of tubular scaffolds for the mimicry of the tunica media. Importantly, the 5 key consideration points were achieved with co-cultures on the bi-layered vascular graft, and showed the correct phenotypes after cellular characterization. Also, the in vitro functionality of the endothelium was proven by its anti-coagulative potential and with the synthesis of NO, indispensable for the communication between the two cell layers. Finally, we want to emphasize that neither soluble factors nor a surface functionalization of the scaffolds was needed for achieving these results, underlining that heterotypic scaffold design that offers biomimetic morphology for more than one cell type within one construct, is a powerful trigger for biofabrication of tissue analogues.

The translation into the application, which includes mechanical optimization of the constructs and further maturation of the cellular phenotypes, is the focus of ongoing studies where the technology is extrapolated to more compliant polymers and where hemodynamic forces stimulate the maturation, to ultimately direct the evolution of a functional vascular graft into a neovessel. Future work will also explore whether the heterotypic design can be exploited to also modulate the immune response towards a regenerative one.

\section{Acknowledgement}

Tomasz Jungst, Iris Pennings and Jürgen Groll, Debby Gawlitta share equal contribution to this work. This research was partially supported by an NWO (Netherlands Organization for Scientific Research) Graduate Program Grant (022.005.018), by the European Research Council (grant number 617989 Design2Heal). We thank Simon Zabler for help with the nanoCT measurements, Joao Pedro Garcia for help with imageJ analysis and Daimon Hall (carbonandneon.com) for support with graphical design. 


\section{Experimental section}

\subsection{Scaffold fabrication}

\subsubsection{Solution electrospinning}


measured in Chloroform against a poly (methyl methacrylate) (PMMA) standard, Corbion, Netherlands) was dissolved in $0.9 \mathrm{~mL}$ of hexafluoroisopropanol (Sigma) for 12 hours in an incubator at $37^{\circ} \mathrm{C}$ and transferred into a syringe $(1 \mathrm{~mL}, \mathrm{BBraun})$ equipped with a flattipped hypodermic needle (27G needle, Stericam, BBraun). Solution electrospinning was performed with a setup composed of a syringe pump (wpi Ltd), a grounded rotating collector, and a high voltage source. The high voltage (12 kV) was applied to the needle tip and the fibers were collected onto a rotating cylindrical target with a diameter of $3 \mathrm{~mm}$ and a length of $20 \mathrm{~cm}$ placed in a distance of $18 \mathrm{~cm}$ from the charged nozzle. The feedrate of the dissolved $\mathrm{PCL}$ was $0.5 \mathrm{~mL} / \mathrm{h}$ and spinning was performed for 14 minutes per mandrel while rotating the mandrel at $120 \mathrm{rpm}$.

\subsubsection{Melt electrowriting}

The nonwoven mesh, still attached to the cylindrical collector, was transferred to the custom-made melt electrowriting device for further processing. The same polymer as used for solution electrospinning was molten $\left(89^{\circ} \mathrm{C}\right)$ in a syringe equipped with a flat tipped spinneret (22G, Precision needle, Nordson EFD) and dispensed with a pressure of 0.7 bar. A voltage of $4.57 \mathrm{kV}$ was applied to the tip, and the nozzle was placed with a distance of 4 $\mathrm{mm}$ above the nonwoven mesh. Combining rotation and translation of the collector, $\mathrm{PCL}$ fibers could be deposited onto the solution electrospun layer with a predefined winding angle. After melt electrowriting the samples were removed from the collector for further processing.

\subsubsection{Scaffold characterization (SEM/porosity)}

The scaffolds were sputter coated with an $8 \mathrm{~nm}$ platinum coating (EM ACE600, Leica) and analyzed via SEM (Crossbeam 340, Carl Zeiss). The winding angle, the diameter of the solution electrospun and the melt electrowritten fibers were measured based on the images using imageJ at 10 different locations respectively. The porosity of the solution electrospun nonwoven meshes was analyzed at the European Synchrotron Radiation Facility using the ID19 beamline with a voxel size of $0.16 \mu \mathrm{m}$. The data was processed using imageJ to calculate the porosity of the scaffolds.

\subsubsection{Mechanical testing}

Mechanical testing of the scaffolds with a $70^{\circ}$ winding angle was performed with a mechanical tester (Z010, Zwick/Roell) using a $100 \mathrm{~N}$ load cell. The burst pressure was 
analyzed using balloon catheters (UROMED) with an initial diameter of $2.7 \mathrm{~mm}$. The burst pressure was detected with a manometer $(\mathrm{N}=5)$.

\subsection{Isolation of MSCs}

Bone marrow aspirates (approved by the local medical research ethics committee, University Medical Center Utrecht) were used for MSC isolation as described previously [81] (four different donors, hereafter referred to as MSC1 - 4, female age 19; female age 74; male aged 60; female aged 20). Researchers were blinded to the medical history of the donors. Aspirates were diluted with phosphate-buffered saline (PBS) $(1: 1)$ and filtered through a $100 \mu \mathrm{m}$ cell strainer. Diluted aspirates were centrifuged $(415 \times \mathrm{g})$ on a Ficoll-Paque (GE Healthcare, Uppsala, Sweden) gradient (density $1.077 \mathrm{~g} / \mathrm{mL}$ ) to retrieve the mononuclear cell (MNC) layer. MNCs were cultured $\left(250,000\right.$ cells $\left./ \mathrm{cm}^{2}\right)$ in expansion medium consisting of a-Minimum Essential Medium (Gibco Paisley, 22561), supplemented with $10 \%$ heatinactivated Fetal Bovine Serum (FBS) (HI biowest, S14068S1810), $100 \mathrm{U} / \mathrm{mL}$ penicillin and $100 \mu \mathrm{g} / \mathrm{mL}$ streptomycin (PenStrep, Gibco, Grand Island, USA), $0.2 \mathrm{mM}$ L-ascorbic acid-2phosphate (ASAP, Vitamin C) (Sigma, A8960), and $1 \mathrm{ng} / \mathrm{mL}$ basic fibroblast growth factor (bFGF-2; R\&D Systems). Cells from passage 4 were used in the experimental set-ups.

\subsubsection{Characterization of MSCs}

Multipotency of the 4 used MSCs was examined via differentiation toward adipogenic, osteogenic, and chondrogenic lineages as described previously [81]. Moreover, MSCs were phenotypically characterized by surface marker expression profiles with flow cytometry (BD Canto II analyzer). Expression of CD90 (THY1, FITC-conjugated; Abcam, ab124527), CD73 (AD2, PE-conjugated; Abcam, ab157335), CD105 (MEM-226, APCconjugated; Abcam, ab60902) was confirmed as well as the absence of CD34 (4H11, APCconjugated; Abcam, ab155377), CD45 (MEM-28, PEC-conjugated; Abcam, ab134202), CD97a (HM47, PE-conjugated; Abcam, ab177274) and CD14 (RPA-M1, FITC-conjugated, Abcam, (ab86896). IgG-matched controls were purchased from Abcam (APC, ab91358; PE, ab37392 and FITC, ab37393). Characterization data is not shown.

Perivascular properties of the progenitor cells was observed by the surface marker CD146 (P1H12 (RUO), bv711 conjugated, BD biosciences 563186) for 3 MSC donors and compared to the matched isotype control (X40 (RUO, k Isotype, BD biosciences 563044).

\subsection{Isolation of ECFC}

Cord blood of 1 donor (aspiration procedure was approved by the medical research ethics committee, University Medical Center Utrecht) was used as a source for the ECFC isolation as described previously [81]. Cord blood was diluted with 2 mM PBS/EDTA (1:3) and centrifuged $(400 \times \mathrm{g}$ ) on a Ficoll-Paque (GE Healthcare) gradient (density $1.077 \mathrm{~g} /$ $\mathrm{mL}$ ). MNCs were plated in a collagen type I (BD Biosciences, rat tail)-coated 6 wells plate 
with endothelial growth medium-2 (EGM-2). Endothelial growth medium was used for subsequent cell expansion, containing Endothelial Basal Medium-2 (Lonza, Walkersville MD, USA) supplemented with 10\% heat inactivated FBS (HI biowest, S14068S1810), $100 \mathrm{U} /$ $\mathrm{mL}-100 \mu \mathrm{g} / \mathrm{mL}$ PenStrep and SingleQuots (Lonza, Walkersville MD, USA).

\subsubsection{Characterization of ECFCs}

Phenotypic characterization of ECFCs was hour performed using a BD FACSCanto II Flow Cytometer. Cells were detached using accutase and checked for the presence of the following endothelial makers: CD31 (AC128, FITC-conjugated; MACS, 130-098-171), VEGFR2 (ES8-20E6, PE-conjugated; MACS, 130-098-905), CD34 (4H11, APC-conjugated; Abcam, ab155377) and CD105 (MEM-226, APC-conjugated; Abcam, ab60902), as well as absence of CD133 (AC133, FITC conjugated; MACS, 130-105-226), CD45 (MEM-28, PECconjugated; Abcam, ab134202) and CD14 (RPA-M1, FITC-conjugated, Abcam, (ab86896). Characterization data is not shown.

\subsection{Scaffold seeding}

Bi-layered scaffolds and SES-only scaffolds were gamma-sterilized, cut in pieces of $\sim 3 \mathrm{~mm}$ in length and immersed in $70 \%$ ethanol followed by 3 times washing in PBS. To promote protein absorption, scaffolds were immersed in medium with $10 \%$ FBS for at least 3 hours at $37^{\circ} \mathrm{C}$, prior to cell seeding. Next, cells were seeded on the scaffold with a density of 4,000 cells $/ \mathrm{mm}^{2}$ (cell suspension concentration $12 \times 10^{6}$ cells $/ \mathrm{mL}$ for MSCs and $24 \times 10^{6}$ cells $/ \mathrm{mL}$ for ECFCs), for 1 hour at $37^{\circ} \mathrm{C}$, resulting in approximately 120,000 seeded cells/scaffold. The seeding efficiency of both the ECFCs $(46.09 \pm 12.21) \%$ and MSCs (55.64 \pm 13.31)\% was determined via DNA quantification of seeded scaffolds after 16 hours, compared to $100 \%$ of seeded cells (Supplementary Figure 2.5). For cell seeding, three groups were made: i) scaffolds mono-seeded with MSCs on the outer layer (group M) ii) scaffolds seeded with ECFCs into the luminal side (group E) or iii) co-seeded scaffolds with ECFCs and MSCs (group ME). Seeding was performed as follows: for group $\mathrm{M}$ i), the scaffolds were placed horizontally on a sterile petridish and $1 / 4^{\text {th }}$ of the total cell amount was pipetted on each quarter. Scaffolds were rotated $1 / 4^{\text {th }}$ every 15 minutes. For group E ii), the total amount of ECFCs was seeded inside the, with medium filled, lumen and immediately rotated to distribute the cell suspension. Hereafter, the scaffolds were rotated $1 / 4^{\text {th }}$ every 15 minutes. For the co-cultured group ME iii), 14 day-cultured scaffolds from group $M(i)$ were placed in a petridish and were seeded with ECFCs as described for group E till day 17 (ii). To confirm reproducibility, groups M (i) and group ME (iii) had independent biological replicates with $n=4$ or $n=3$ different MSC donors respectively (MSC1-4), together with experimental duplicates $(n=2)$ for each outcome parameter. One donor of the ECFC was used in all experiments. 


\subsubsection{Scaffold cultures}

All groups were vertically cultured in 48-wells cultured plates with $0.5 \mathrm{~mL}$ of the desired medium. Group M (ii) was cultured in MSC expansion medium (a-Minimum Essential Medium, 10\% heat-inactivated FBS, PenStrep, ASAP and $1 \mathrm{ng} / \mathrm{mL}$ bFGF) for 7 or 14 days. Group E (ii) and group ME (iii) were cultured in EGM-2 medium and cultured for (ii) 7 and (iii) 17 days ( 14 day MSC mono-culture in MSC expansion medium, followed by 3 days coculturing). After culture time, the discs were either fixated in 10\% Formalin solution or $6 \%$ glutaraldehyde (for SEM), or used for RNA isolation. For MSC control situations, MSC1 was cultured in a 12 wells culture- plate (seeding density 1500 cells $/ \mathrm{cm}^{2}$ ) for 3,7 or 14 days in MSC expansion medium, supplemented with $1 \mathrm{ng} / \mathrm{mL}$ bFGF, after which the monolayer was either fixed or used for RNA isolation.

\subsection{SEM}

Scaffolds were washed in PBS and fixated in $6 \%$ glutaraldehyde in PBS $\left(25 \%\right.$ in $\mathrm{H}_{2} \mathrm{O}$, G6257, Sigma Aldrich) on ice, for 15 minutes. Next, samples were washed twice with PBS on ice for 10 minutes, after which the samples were dehydrated at room temperature, each step for 10 minutes (2x 70\%, 2x 90\%, 2x 100\%). Afterwards, samples were incubated twice with Hexamethyldisilazane (HMDS) (440191, Sigma Aldrich) for 15 minutes, and air dried afterwards. After the fixation, scaffolds were sputter-coated with a platinum coating (4 nm, EM ACE600, Leica) and analyzed via SEM (Crossbeam 340, Carl Zeiss).

\subsection{Orientation of the vSM-like cells}

To assess the influence of the winding angle on the orientation of the MSCs, whole mount immunofluorescent staining was performed for F-actin (Sigma, Phalloidin Fluorescein Isothiocyanate labeled; P5282, $0.2 \mu \mathrm{M}$ ) on group $\mathrm{M}$ (i), after 7 days, with varying winding angles (no MEW layer - $30-70^{\circ}$ ). Of every scaffold with specific winding angle, seeding was independently repeated with 4 different MSC donors (MSC1-4) $(n=2)$ to confirm reproducibility. Confocal microscopy (Leica DMi8) was used for imaging. 3D Z-stacks were made from the outer MEW layer of 3 locations per sample, Z-stack settings were set for every sample individually but with the same amount of images (21) for all samples. Maximum projections were made with ImageJ $1.47 \mathrm{v}$ and converted into 8 -bit followed by loading in the orientation plugin (min. coherency $80 \%$ - min. energy 10\%). Orientation of the cells were quantified in the maximum projections of the 3D stacks made from the total MEW layer (P1) only the bottom (P2) or only the upper (P3) zone of the MEW layer. Graph 1 in Figure 2.3 shows values of P1 (no difference was found between P1-P2-P3) (Supplementary Figure 2.10).

\subsection{Fluorescent stainings}

\subsubsection{ECM and vascular smooth muscle cell markers}

To demonstrate that the seeded MSCs and their extra cellular matrix showed a vSMC phenotype, fixated samples were cut in smaller pieces, permeabilized ( 15 minutes in $0.2 \%$ 
Triton-X/PBS) and blocked (15 minutes in $5 \%$ Bovine Serum Albumin (BSA) in PBS) for nonspecific protein binding. Primary antibodies for Laminin subunit a5 (clone 4B12, MABT39, Merck Millipore $0.015 \mathrm{mg} / \mathrm{mL}$ ) and Collagen IV (ab769 Millipore, $0.04 \mathrm{mg} / \mathrm{mL}$ ) were incubated for one hour at room temperature (with matched isotype controls). Washing steps were performed with $0.1 \%$ Tween in TBS, after which the matching secondary antibody incubation (GaM Alexa 546 or DaG, life technologies $4 \mu \mathrm{g} / \mathrm{mL}$; DaG Alexa 546, Life Technologies, 2 $\mu \mathrm{g} / \mathrm{mL}$ ) was carried out for one hour at room temperature. Calponin (CALP, MU333-UC, Biogenex, $0.15 \mathrm{mg} / \mathrm{mL}$ ) staining was performed with the $A B C$ method, with sheep anti-mouse biotinylated (1:300, RPN1001v1, GE Healthcare) as secondary antibody and as tertiary antibody Streptavidin, Alexa Fluor ${ }^{\circledast} 488$ Conjugate (S32354, Life technologies, $0.005 \mathrm{mg} / \mathrm{mL}$ ), together with a-Smooth Muscle Actin (a-MA, Clone 1A4, Cy3 $0.5 \mu \mathrm{g} / \mathrm{mL}$, C6198, Sigma Aldrich) for costaining. Nuclei were counterstained with 4,6-diamidino-2-phenylindool $100 \mathrm{ng} / \mathrm{mL}$ (DAPI, Sigma) for 15 minutes and washed with PBS and visualized by confocal imaging (Leica DMi8).

\subsubsection{Endothelial markers}

To demonstrate that the seeded ECFCs contained the endothelial phenotype in the monolayer, immunofluorescence staining for CD31 $(0.13 \mathrm{mg} / \mathrm{mL}$ mouse anti-human CD31, M0823, Dako) (ABC method and antibodies as described above), von Willebrand Factor (vWF) (3E2D10, Abcam 194405, 0,5 $\mu \mathrm{g} / \mathrm{mL}$ ) and vascular endothelial cadherin (VE-cadherin) (D87F2, Cell signal, 2500S, 1:250) was conducted. After permeabilization, non-specific protein binding was blocked followed by one hour of incubation with the primary antibodies. After washing with $0.1 \%$ Tween in TBS, one hour of incubation with the secondary antibodies, GaM Alexa 546 life technologies - A-11003, $0.004 \mathrm{mg} / \mathrm{mL}$ or GaR HiLyte Fluor 488, $0.001 \mathrm{mg} / \mathrm{mL}$ respectively, was performed. Finally, nuclei were counterstained with DAPI (100 ng/mL, Sigma) for 15 minutes at room temperature. Staining was visualized by confocal imaging (Leica DMi8).

\subsection{RNA isolation}

Total cellular RNA was extracted by resuspending in $0.5 \mathrm{~mL}$ Trizol Reagent (Thermo Fisher Scientific) for 5 minutes at room temperature. Samples were stored at $-80^{\circ} \mathrm{C}$ till further use. RNA was isolated from the samples by mixing the RNA extraction in Trizol with $20 \%$ Chloroform (Millipore) and incubating for 3 minutes at room temperature. Tubes were centrifuged at $12.000 \times \mathrm{g}$ for 15 minutes $\left(4^{\circ} \mathrm{C}\right)$ after which the colorless upper aqueous phase was pipetted off and mixed with an equal amount of $\mathrm{mL} 2$-propanol (Sigma) ( 0.25 $\mathrm{mL}$ ) and $0.5 \mu \mathrm{L}$ glycogen (R0551, Thermo Fisher Scientific). This mixture was incubated for 10 minutes at room temperature and followed by centrifugation at $12.000 \times \mathrm{g}$ for 10 minutes $\left(4^{\circ} \mathrm{C}\right)$. Then, the pellet was washed in $75 \%$ ethanol and centrifuged at $7.500 \times \mathrm{g}$ for 5 minutes $\left(4^{\circ} \mathrm{C}\right)$. The ethanol was aspirated and the pellet was air-dried. Afterwards, the pellet was re-suspended in $10 \mu \mathrm{L}$ of RNase-free water and heated to $55^{\circ} \mathrm{C}$ for 10 minutes, shortly vortexed and put on ice. A DNase step was performed by addition of $1 \mu \mathrm{L}$ of 
Turbo DNase buffer (AM2238, Ambion) and $0.5 \mu \mathrm{L}$ DNase (2 $\mathrm{U} / \mu \mathrm{L})$ per samples, 30 minute incubation at $37^{\circ} \mathrm{C}$ and inactivation for 10 minutes at $75^{\circ} \mathrm{C}$.

\subsubsection{Quantitative polymerase chain reaction (RT-PCR)}

For use in the qPCR, total RNA was first reverse-transcribed into cDNA by means of a iScript cDNA synthesis kit (Biorad, 170-8890). The RNA input concentration was set to the sample with the lowest yield $(0.5 \mu \mathrm{g})$, providing the same cDNA end-concentration for all samples used in this data set. The synthesis reaction was performed according to the manufacturer's instruction under the following cycling conditions: $25^{\circ} \mathrm{C}$ for 5 minutes, 30 minutes at $42^{\circ} \mathrm{C}, 5$ minutes at $85^{\circ} \mathrm{C}$ and hold at $4^{\circ} \mathrm{C}$. The cDNA product was then diluted till an endconcentration of $5 \mathrm{ng} / \mu \mathrm{L}$ with RNase-free water and stored at $-80^{\circ} \mathrm{C}$ till further use.

The qPCR reaction mix was prepared with the FastStart SYBR green master (Sigma, 04673484001) for a reaction volume of $20 \mu \mathrm{L}$ with $10 \mathrm{ng}$ of cDNA and $500 \mathrm{nM}$ per primer (forward and reversed). The qPCR reaction was performed in a Roche LightCycler 96, according to the manufacturer's instruction under the following cycling conditions: Pre-incubation at $95^{\circ} \mathrm{C}$ for 5 minutes, 3-step amplification (50 cycles) of 10 seconds $95^{\circ} \mathrm{C} / 15$ seconds at 60 or $63^{\circ} \mathrm{C} / 30$ seconds at $72^{\circ} \mathrm{C}$.

Samples from group $M($ i) and group ME (iii) were checked for the quantitative expression of mRNA of SMC-specific genes, including aSMA (ACTA2), CALP (CNN1), Smooth Muscle 22a (Transgelin/SM22 a) and MY11 (SMMHC); mature endothelial specific genes, including CD31 (PECAM1), VE-cadherin (CDH5), vWF; ECM specific genes associated with vSMC maturation and basal membrane formation, including Laminin subunit a4 and a5 (LAMA4, LAMA5) and (tropo)elastin (ELN); and/or genes involved in EC-vSMC crosscommunication, including transforming growth factor $\beta$ (TGFB1) and its opposing Activin receptor-like kinase (ALK) type 1 and 5 receptor of the Smad pathways. The most optimal housekeeping gene among a set of candidates was selected according to its' expression stability. The algorithm from Normfinder indicated that the optimal normalization gene for this data set was glyceraldehyde-3-phosphate dehydrogenase (GAPDH). Primer sequence and temperature can be found in Supplementary Table 1.

\subsubsection{Relative gene expression}

For group M (i), comparisons were made for day 3 - 7- 14 for MSCs cultured in a wells plate (control situation 1), cultured on the SES only layer (test situation 1 and control situation 2) and MSCs cultured on the bi-layered scaffold (test situation 2). Relative gene expression was calculated including an efficiency correction for real-time PCR efficiency of the individual transcripts $[175,176]$.

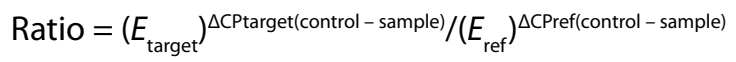


With this, relative gene expression values above 1 indicate higher expression of the mRNA in the samples compared to the control situation. Conversely, relative expression values below 1 indicate less mRNA in the experimental group versus control group. For group ME (iii), no comparisons were made to avoid influence of varying ratios of the cell-types in the co-cultured constructs, especially when comparing to mono-cultures; instead, gel electrophoresis of the qPCR product is shown.

\subsubsection{Gel electrophoresis}

Sample groups (group M) with minimal amounts of target nucleic acid (Ct values between 38 and 42) were checked for amplification of the true sequence of interest by analyzing the fragment length via gel electrophoresis. In short, a 3\% agarose solution was prepared in Tris/Borate/EDTA (TBE) buffer with $0.01 \mu \mathrm{l} / \mathrm{mL}$ sybersafe solution. The qPCR end-products of interest (and corresponding negative $\mathrm{qPCR}$ primer control $\mathrm{H}_{2} \mathrm{O}$ ) were prepared with gel loading dye (Purple (6X), no SDS, NEB B7025). Slots in the solidified gel were filled with $12 \mu \mathrm{l}$ of prepared sample volume or $1 \mu \mathrm{g}$ of the ladder in loading dye (50 bp DNA ladder, NEB N3236S). Images were made with a biorad UV gel reader in the program Quantity One 4.5.2, after running for 45 minutes on 80 Volts at RT.

\subsection{Functionality assays}

\subsubsection{Nitric Oxide assay}

Measurements of the NO concentration in the group ME (iii) were performed on medium stored from the cultures at day 17. To prevent interference of the phenol red, EGM2 medium without phenol red was added to the cultures (group M (ii)) after seeding with the ECFCs (group ME (iii)) at day 14. Medium $(500 \mu \mathrm{L}$ ) was centrifuged at $320 \times \mathrm{g}$ for 5 minutes, the supernatant was stored at $-20^{\circ} \mathrm{C}$ till further use. To measure complete nitrate/nitrite content, the Cayman nitrite/nitrate fluorometric assay kit was used (Cayman, 780051). The protocol was according to the manufacturer. In short, $20 \mu \mathrm{L}$ of sample medium was used in the assay and incubated for 1 hour after addition of the enzyme cofactors and nitrate reductase. In the standard and the blank, also $20 \mu \mathrm{L}$ of EGM2 phenol free medium was used, to correct for its interference. Plate was red at a fluorescent plate reader at excitation 355 and emission 405. Concentration was calculated as total amount of nitrate/nitrite in the reaction mix.

\subsubsection{Platelet adhesion assay}

Endothelial functionality based on its anticoagulation potential was assessed with a platelet binding assay. Blood was obtained from a healthy volunteer in citrate tubes. Blood was spun down at $130 \times \mathrm{g}$ for 15 minutes without brake. The supernatant was collected and spun down once again at $250 \times \mathrm{g}$ for 15 minutes. The platelet containing pellet was resuspended in $1 \mathrm{~mL}$ of its platelet poor plasma (PPP) and counted. Scaffolds seeded with ECFCs at the luminal side (group E) were washed with PBS and incubated 
for 1 hour with $100 \mathrm{uL}$ of $1 \times 10^{8}$ platelets $/ \mathrm{mL}$ at $37^{\circ} \mathrm{C}$. Samples were washed 3 times in PBS and fixated in $10 \%$ Formalin solution. Platelet aggregation was stained for with the mouse anti-human CD41 antibody (Enzo Life Sciences, ALX-805-024-C100, $4 \mu \mathrm{g} / \mathrm{mL}$ ) and the matching secondary antibody (GaM Alexa 546 or DaG, life technologies, $4 \mu \mathrm{g} / \mathrm{mL}$ ). Nuclei were stained with DAPI and images were obtained with confocal laser scanning microscopy (Leica DMi8SP8x).

\subsubsection{Collagen Gel Lattice Contraction Assay}

For measurement of functionality of vSM-like cells, a collagen contraction assay was performed [177]. vSM-like cells, pre-differentiated on scaffolds (group M) and in a wells plate (positive control situation, section 6.4.1, Supplementary Figure 2.11) were detached from the scaffold or well with trypsin after 10 days. Collagen gels $(2 \mathrm{mg} / \mathrm{mL})$ were made with $3.5 \times 10^{5}$ cells $/ \mathrm{mL}$ final cell concentration in 10x PBS and 0.023 times the collagen volume of $\mathrm{NaOH}$. The $450 \mu \mathrm{L}$ collagen solution was pipetted into a 24-wells culture plate and incubated for 1 hour at $37^{\circ} \mathrm{C}$ to polymerize the collagen gel lattices. Gels were gently released from the bottom of the plate with a needle to initiate contraction of the gels and further cultured in MSC expansion medium enriched with bFGF. The contraction of the lattices was monitored with a stereo-microscope (Olympus SZ61) for 48 hours. Empty collagen gels were included as negative controls.

\subsection{Image adaptions}

Images obtained with confocal microscopy (Leica DMi8SP8x) were adjusted with Adobe Photoshop CS6 for intensity of the signal by removing under and overexposed pixels. No local thresholding was performed. Confocal laser power was kept the same within each session and when comparing samples. Images from gel electrophoresis, made with Quantity One 4.5.2, were cropped in Adobe Photoshop CS and contrast was adjusted accordingly.

\subsection{Statistical analysis}

The statistical analysis was performed with the software package Statistica 13 (TIBICO Software Inc.). A t-test was performed on the effective $E^{\triangle C P}$ values Ratio $=\left(E_{\text {target }}\right)^{\Delta C \text { Ptarget(control }}$ - sample) $/\left(E_{\text {ref }}\right)^{\Delta C \text { Pref(control - sample) }}$ of the gene expression to evaluate if the data from the qPCR measurements show a statistically relevant difference from the house keeping gene, both normalized to the control group. 


\section{Supporting information}

Supplementary Table 1. Primer sequences of genes used for $q P C R$.

\begin{tabular}{|c|c|c|c|}
\hline Gene & Forward Primer & Reverse Primer & $\begin{array}{l}\text { Product } \\
\text { length (bp) }\end{array}$ \\
\hline GADPH & 5'-ATGGGGAAGGTGAAGGTCG-3' & 5'-TAAAAGCAGCCCTGGTGACC-3' & 70 \\
\hline $\begin{array}{l}\text { Laminin-10/11 } \\
\text { (a5 subunit) }\end{array}$ & 5'-AGCATCACATTCCTGGAGCC-3' & 5'-GTCTCCGTATGCCGGAAGTT-3' & 95 \\
\hline $\begin{array}{l}\text { Laminin-8/9 } \\
\text { (a4 subunit) }\end{array}$ & 5'-GACGCCAGGATAGCCAAGAA-3' & 5'-GGGGGTTCAAAACCTTCTTCC-3' & 98 \\
\hline Elastin & 5'-GCCAAAGCCGCCCAGTTT-3' & 5'-CGCCGACACCAGGAACTAAC-3' & 40 \\
\hline TGF- $\beta 1$ & 5'-GAGCCGTGGAGGGGAA-3' & 5'-GCCGGTAGTGAACCCGT-3' & 103 \\
\hline ALK1 & 5'-AGGGCAAACCAGCCATTG-3' & 5'-GGTTGCTCTTGACCAGCACAT-3' & 63 \\
\hline ALK5 & 5'-TCCCAAACAGATGGCAGAGC-3' & 5'-CCTAGCTGCTCCATTGGCAT-3' & 86 \\
\hline CD31 & 5'-GCAGTGGTTATCATCGGAGTG-3' & 5'-TCGTTGTTGGAGTTCAGAAGTG-3' & 137 \\
\hline VE-cadherin & 5'-GGCAAGATCAAGTCAAGCGTG-3' & 5'-ACGTCTCCTGTCTCTGCATCG-3' & 101 \\
\hline vWF & 5'-CGACGGGCTCAAATACCTGT-3' & 5'-CCGAAAGGTCCCAGGGTTAC-3' & 85 \\
\hline a-SMA & 5'-CCAACTGGGACGACATGGAA-3' & 5'-GGGCAACACGAAGCTCATTG-3' & 63 \\
\hline Calponin & 5'-GGTGAAGCCCCACGACATT-3' & 5'-GTTCACCTTGTTTCCTTTCGTCTT-3' & 121 \\
\hline SM-MHC & 5'-ATTTGGACAACCAGCGGCAA-3' & 5'-AGAGATGTTTTTCTCCTCGGCT-3' & 89 \\
\hline SM22a & 5'-AGTGCAGTCCAAAATCGAGAAG-3' & 5'-CTTGCTCAGAATCACGCCAT-3' & 154 \\
\hline
\end{tabular}





Supplementary Figure 2.1 Anatomy and organization of a native vessel. A) Organization of the vessel in 3 layers, including the tunica intima, tunica media and tunica adventitia shown with hematoxylin and eosin staining. B) vSMCs in the tunica media are positive for calponin and C) SMMH and D) structures in the tunica intima and tunica media are positive for laminin subunit $\mathrm{a} 5$ and E) collagen type IV. f) Also, aSMA-positive vSMCs are seen in the tunica media.



Supplementary Figure 2.2 NanoCT reconstruction of the two layered samples in cross section view. Thick fibers on the top are made with melt electrowriting. The thin fibers on the lower part of the images are solution electrospun fibers. 




Supplementary Figure 2.3 The winding angle $\alpha$ is defined as the angle between the axis of rotation of the collector during fabrication and the fibers.

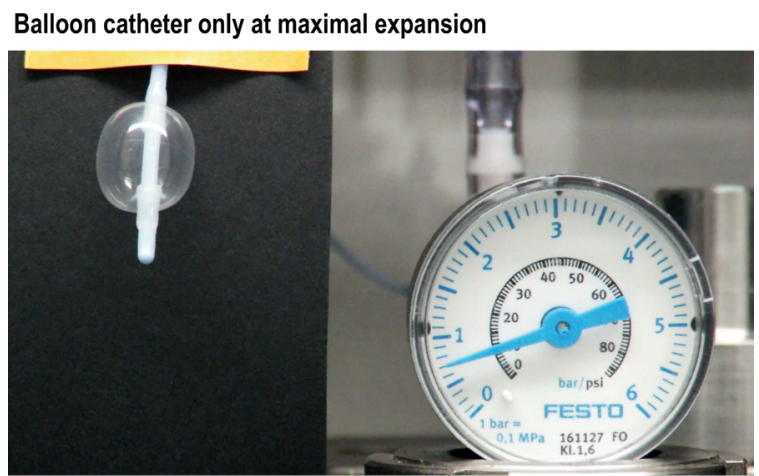

Balloon catheter and scaffold before burst

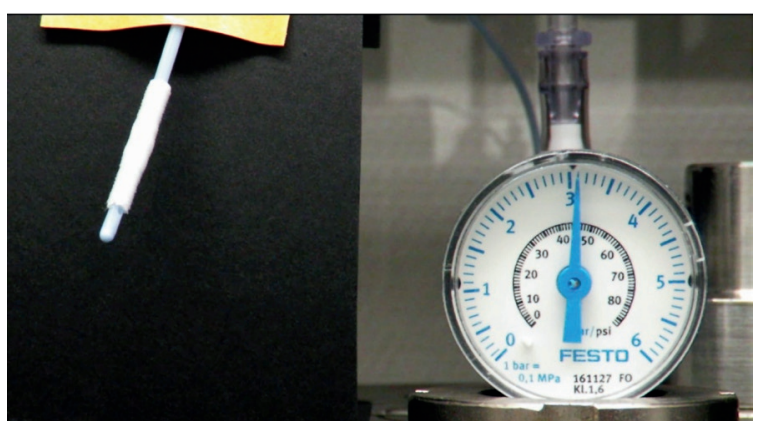

Supplementary Figure 2.4 Balloon-catheter-based burst tests. As the scaffold without the cells is not gas tight, a balloon inflation test needed to be performed. The balloon itself expands at pressure of 0.7 bars. With the scaffold, a burst pressure of over 3 bars could be reached. 


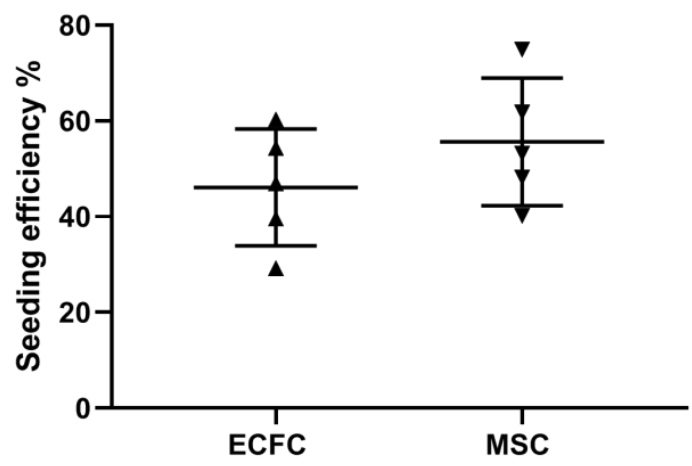

Supplementary Figure 2.5 Seeding efficiency of ECFCs and MSCS on the scaffold. Efficiency was determined with DNA quantification 16 hours after seeding for ECFCs $(46.09 \pm 12.21) \%$ and MSCs (55.64 \pm 13.31$) \%$ individually, compared to $100 \%$ of seeded cells.
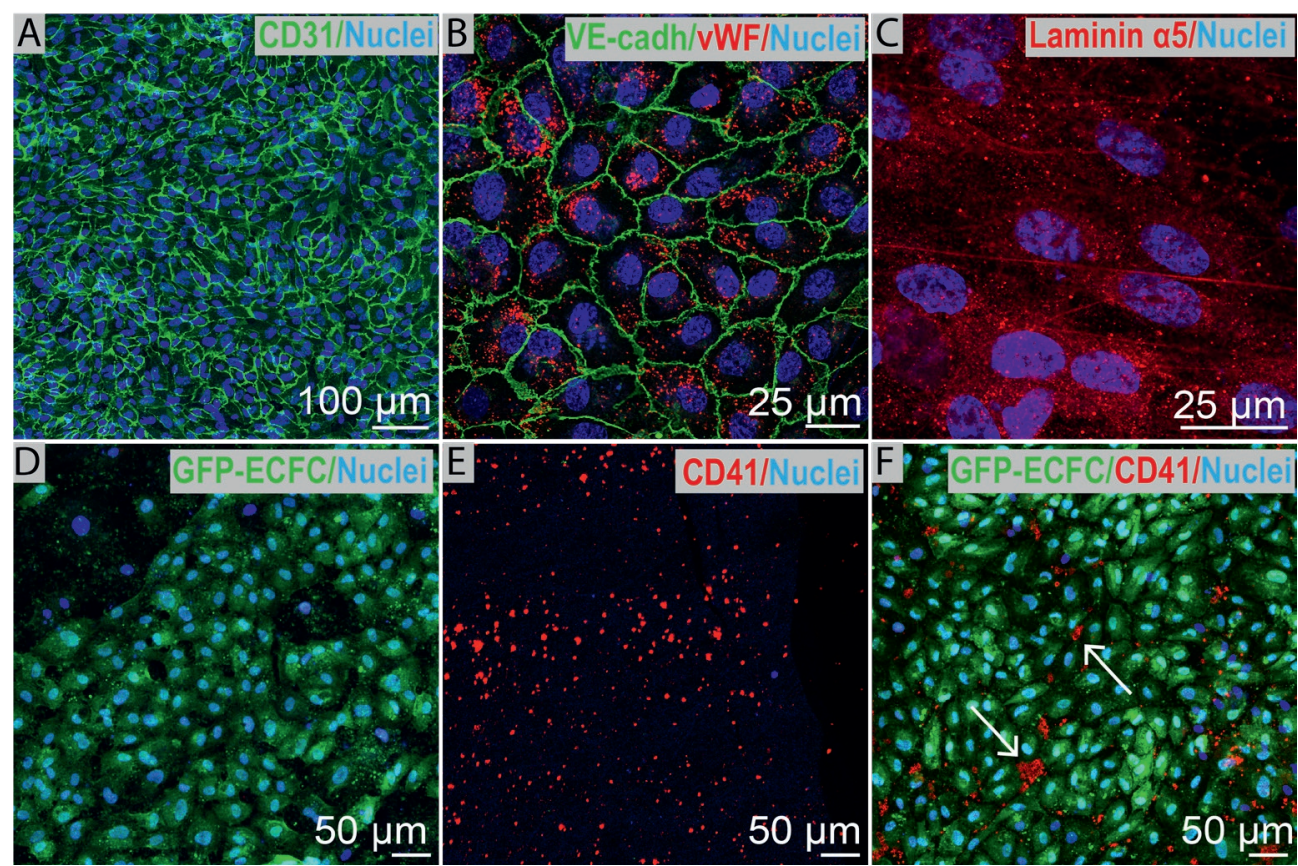

Supplementary Figure 2.6 The SES layer facilitates endothelialization in mono-culture. A) Large area of confluent CD31-positive mono-layer, also positive for B) VE-cadherin and VWF after 7 days of mono-culture. C) Laminin a5 subunits were found surrounding the individual ECFCs, indicating formation of a basement membrane. The anti-thrombotic capacity of the endothelium was proven by a platelet adhesion assay. D) GFP-ECFCs in a monolayer on the scaffolds without added platelets after 7 days, E) Platelets (CD41) adhered to a non-seeded scaffold, and F) to the exposed scaffold in the gaps between endothelial cells (white arrows). No adhesion of platelets to the endothelial cells was observed. 



Supplementary Figure 2.7 Co-cultured constructs keep desired phenotypes. A-C) Gene expression of markers for vSMCs (calponin, aSMA, SM22a), ECM (laminin a4 and a5 subunit, elastin), endothelial layer (CD31, VE-cadherin, vWF) and cross-communication (Alk1/Alk5/TGF $\beta$ pathway) in co-cultures from ECFCs with 3 different MSC donors (a-b-c) after 17 days of culture. $\mathrm{H}_{2} \mathrm{O}$ control represents a mix of the individual $\mathrm{H}_{2} \mathrm{O}$ controls for each specific primer. D) The vSMC markers, aSMA and calponin, were present on the protein level and did not differ from the expression in group $M$ of Figure $4 \mathrm{E}-\mathrm{G}$.
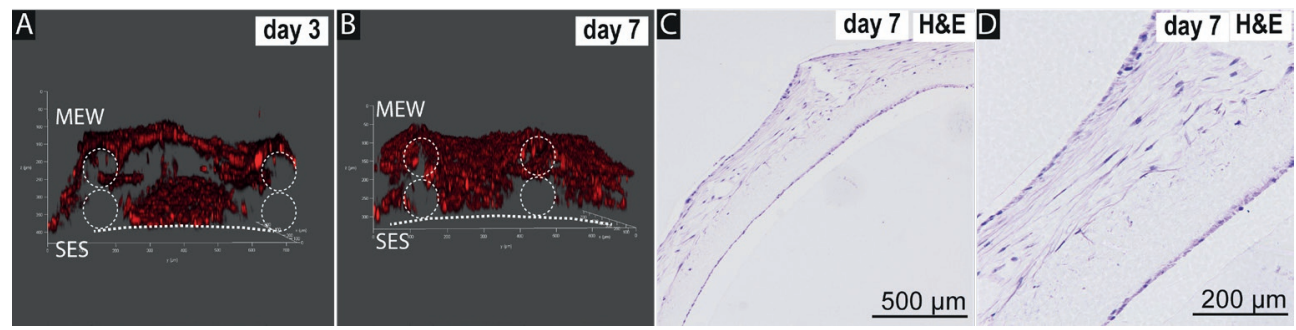

Supplementary Figure 2.8 Infiltration and proliferation of MSCs in MEW medial layer, shown in crosssectional view. A) MSCs were labeled with Dil (Thermo Fisher) and imaged over time. MSCs attached and proliferated on SES interface and MEW fibers after seeding and B) proliferated into a multilayered fashion between the MEW fibers after 7 days. C-D) the stacked cells show an elongated morphology in a circumferential orientation. 

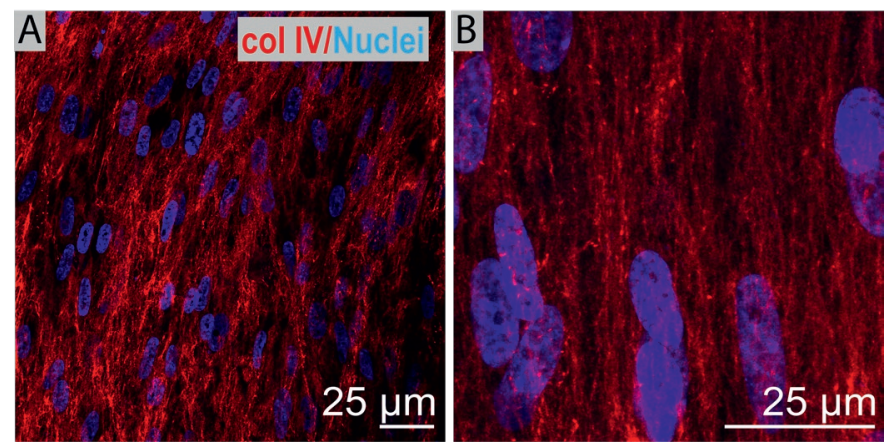

Supplementary Figure 2.9 Basement membrane components of vSM-like cells on the bi-layered vessel. The vSM-like cells are surrounded by A) collagen type IV-positive structures that follow the cellular orientation and B) embrace the individual cells

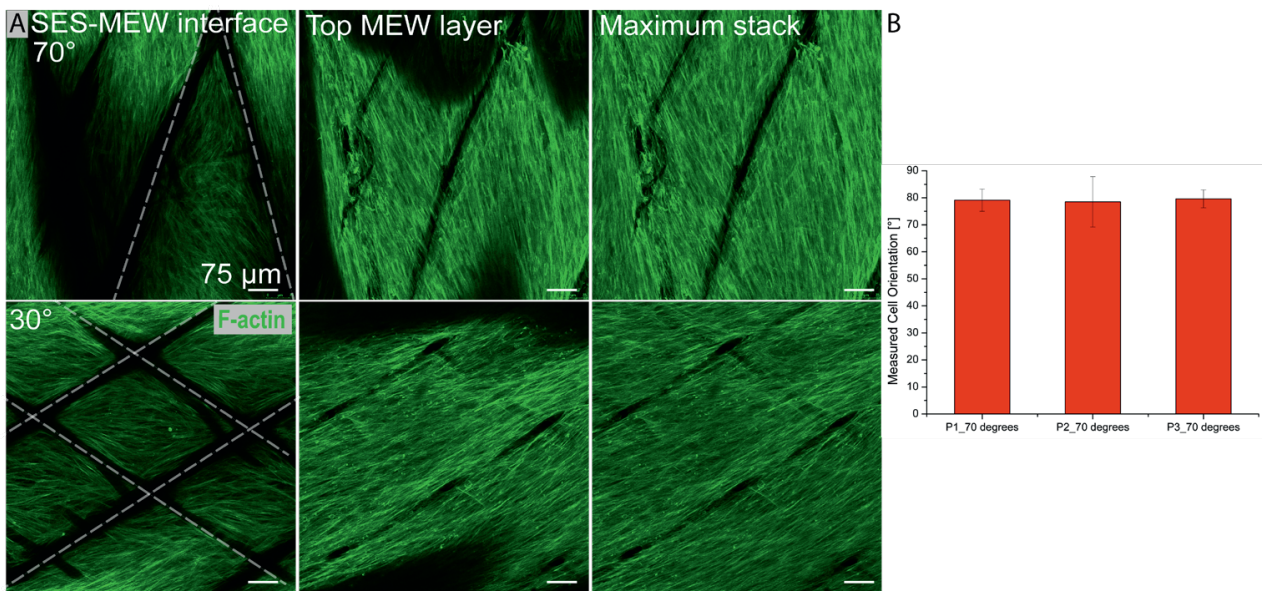

Supplementary Figure 2.10 Orientation of cellular alignment in the whole thickness of the MEW layer. A) Representative images of F-actin stained cells after seeding and culture for 7 days on different levels in the MEW layer; the left column shows a single image of the cell layer directly on the SES interface, the middle column shows a projection of the top part of the MEW layer, and the right column shows a projection of the complete stack of the whole MEW layer. A comparison of the different columns reveals that only the cells in direct contact with the SES layer do not follow the orientation of the MEW fibers but reflect the orientation along the scaffold's long axis that they also show when seeded on the nonwoven-only. The majority of the cells is influenced by the MEW fibers and align according to the orientation of the MEW fibers. This was shown by the analysis of the orientation of the different in different locations of the MEW layer. The orientations of the cells were quantified in the maximum projections of the 3D stacks made from the total MEW layer (P1) at the bottom (P2) or only the upper (P3) zone of the MEW layer. B) Projections were used to determine the mean cell orientations. No difference in cell orientation was found between P1-P2-P3, in 4 MSC donors ( $n=2$ samples, $n=3$ location per sample). Dotted lines represent the MEW fibers. 

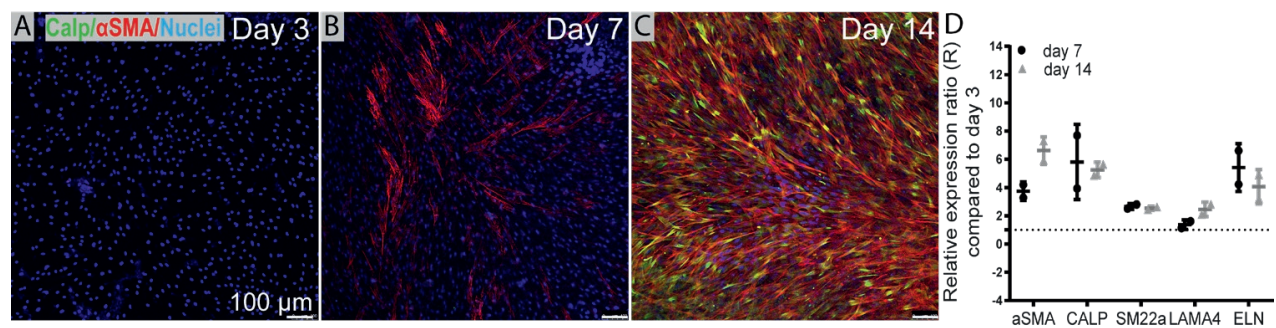

Supplementary Figure 2.11 Confluency-driven MSC differentiation towards vSM-like cells. A) BMderived MSCs cultured in well plates were aSMA/Calponin after 3 days of culture, then $B$ ) turning into $\mathrm{SSMA}^{+} /$Calponin ${ }^{+}$after reaching confluency after 7 days and had $\mathrm{C}$ ) increased protein levels of $\mathrm{aSMA}^{+} /$Calponin $^{+}$after 14 days, accompanied with multi-layered cells in a typical 'hill and valley' orientation. D) Relative gene expression levels confirmed the increase of marker expression associated with the vSMC phenotype at day 7 and day 14 , compared to day 3 .
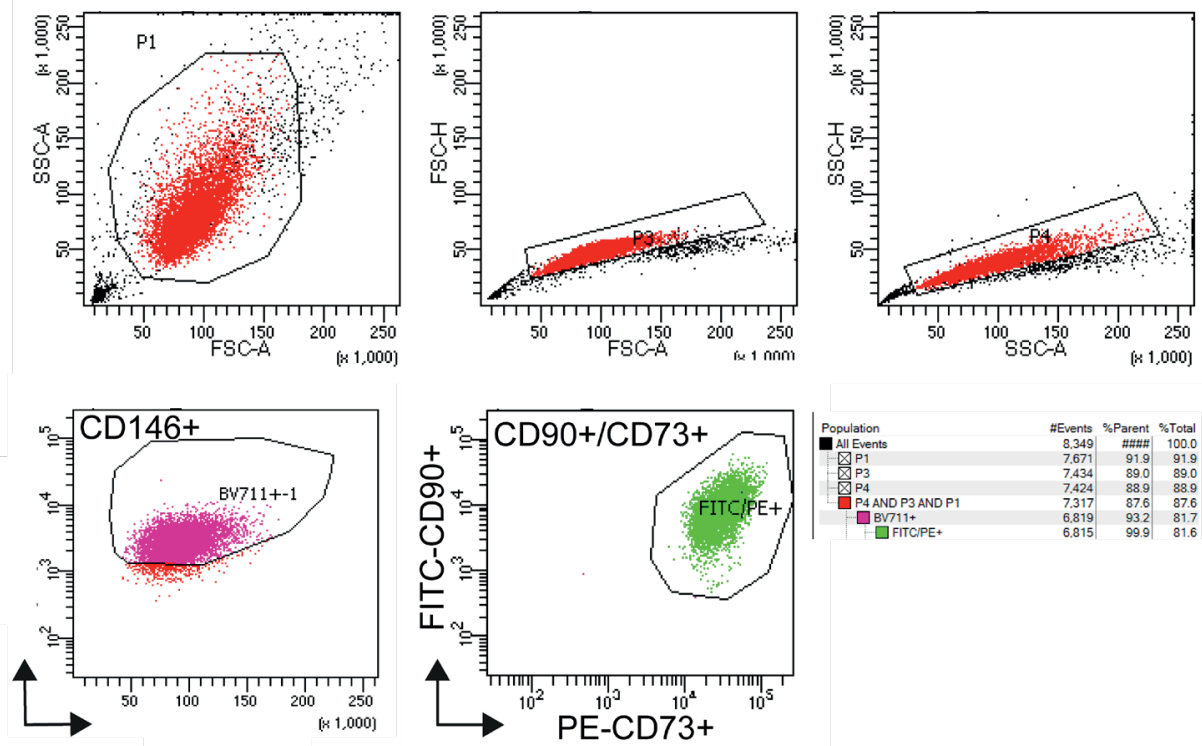

Supplementary Figure 2.12 Presence of the perivascular marker CD146 on MSCs. With FACS the presence of $\mathrm{CD} 146^{+}(\mathrm{BV} 711)$ marker on the MSC was confirmed compared to the correct isotype. $99 \%$ of the $\mathrm{CD}_{146}{ }^{+}$cells were also $\mathrm{CD} 90^{+} / \mathrm{CD} 3^{+}$, normally used as standard MSC characterization markers. Representative data shown for one of the 3 used MSCs. 

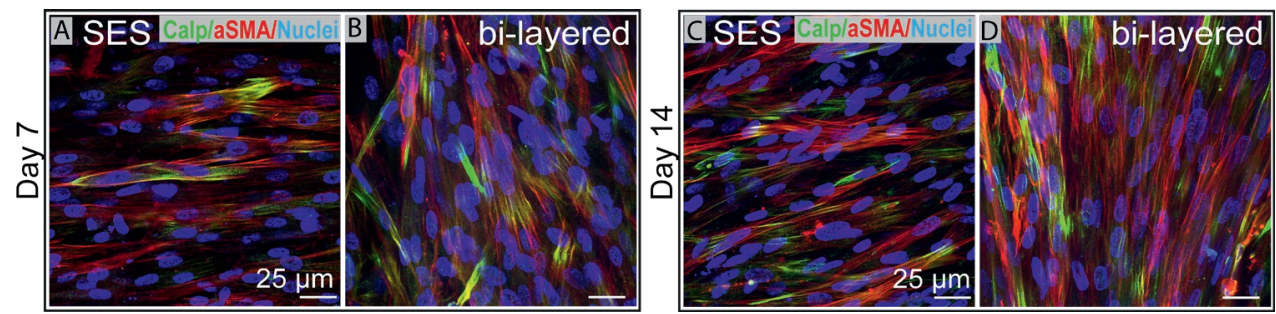

Supplementary Figure 2.13 Differentiation and orientation of vSM-like cells. A) vSM-like cells cultured on the SES only layer after 7 days show an orientation in the longitudinal direction in a single layer, compared to the vSM-like cells on the B) bi-layered scaffold where stacked cells align in a circumferential orientation. The double positive aSMA/Calponin staining of cytoskeleton is clearly visible. After 14 days of culture the vSM-like cells on the C) SES only layer increased their confluence and the number of aSMA ${ }^{+} /$Calponin ${ }^{+}$cells, and show a shift in orientation. D) The cells on the bilayered graft show an increased elongation and orientation.

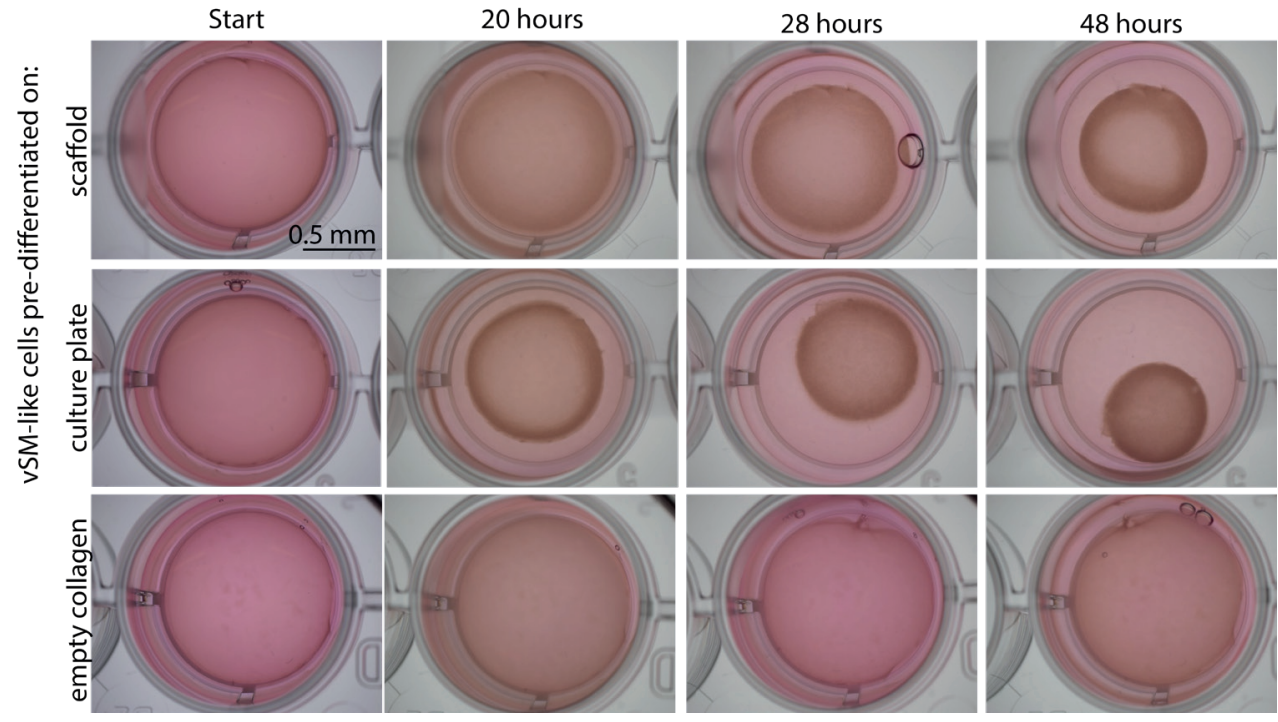

Supplementary Figure 2.14 vSM-like cells were characterized by contraction of a collagen gel. MSCs were pre-differentiated on the scaffolds or on a culture plate for 10 days. They were then seeded in collagen gels, which show considerable contraction after 48 hours, compared to the empty collagen gels. 


\section{Layer-specific cell differentiation in bi-layered vascular grafts under flow perfusion}

this chapter is based on: Layer-specific cell differentiation in bi-layered vascular grafts under flow perfusion.

I. Pennings*, E.E. van Haaften*, T. Jungst, J.A. Bulsink, A.J.W.P Rosenberg, J. Groll, C.V.C. Bouten, N.A. Kurniawan, A.I.P.M. Smits, D. Gawlitta Biofabrication, 2019. 12(1). * these authors contributed equally to this work 


\section{Abstract}

Bioengineered grafts have the potential to overcome the limitations of autologous and non-resorbable synthetic vessels as vascular substitutes. However, one of the challenges in creating these living grafts is to induce and maintain multiple cell phenotypes with a biomimetic organization. Our biomimetic grafts with heterotypic design hold promises for functional neovessel regeneration by guiding the layered cellular and tissue organization into a native-like structure. In this study, a perfusable two-compartment bioreactor chamber was designed for the further maturation of these vascular grafts, with a compartmentalized exposure of the graft's luminal and outer layer to cell-specific media. We used the system for a co-culture of endothelial colony forming cells and multipotent mesenchymal stromal cells (MSCs) in the vascular grafts, produced by combining electrospinning and melt electrowriting. It was demonstrated that the targeted cell phenotypes (i.e., endothelial cells (ECs) and vascular smooth muscle cells (vSMCs), respectively) could be induced and maintained during flow perfusion. The confluent luminal layer of ECs showed flow responsiveness, as indicated by the upregulation of COX-2, KLF2, and eNOS, as well as through stress fiber remodeling and cell elongation. In the outer layer, the circumferentially oriented, multi-layered structure of MSCs could be successfully differentiated into vSM-like cells using TGF $\beta$, as indicated by the upregulation of aSMA, calponin, collagen IV, and (tropo)elastin, without affecting the endothelial monolayer. The cellular layers inhibited diffusion between the outer and the inner medium reservoirs. This implies tightly sealed cellular layers in the constructs, resulting in truly separated bioreactor compartments, ensuring the exposure of the inner endothelium and the outer smooth muscle-like layer to cell-specific media. In conclusion, using this system, we successfully induced layer-specific cell differentiation with a native-like cell organization. This co-culture system enables the creation of biomimetic neovessels, and as such can be exploited to investigate and improve bioengineered vascular grafts. 


\section{Introduction}

Replacements of small diameter blood vessels $(<6 \mathrm{~mm})$ are in clinical demand, for example in coronary artery bypass grafting and for hemodialysis procedures [46]. Autologous conduits are the current clinical gold standard substitute because of their biocompatibility, anti-thrombogenic properties, and match of mechanical properties with the native vessels [178]. However, autologous conduits are restricted by limited material availability, e.g. due to existing vascular disease or previous harvest. As a surrogate, synthetic vessels are frequently used in these procedures [44]. Nevertheless, synthetic vessels often occlude in vivo due to the development of intimal hyperplasia, i.e., progressive smooth muscle cell ingrowth and proliferation, eventually leading to stenosis $[45,46,178]$. To overcome these issues and to address the clinical need for vascular substitutes, bioengineered grafts have been proposed as an alternative [179].

Bioengineered grafts should mimic the native vessel structure with the appropriate tissue characteristics to withstand the hemodynamic in vivo environment. The inherent architecture of a native vessel comprises at least a luminal layer (tunica intima), which is lined with an anti-thrombogenic monolayer of endothelial cells (ECs), and a secondary medial layer (tunica media) containing stacked smooth muscle cells (SMCs) with a concentric organization that is critical for vasoconstriction and vasodilatation [180]. Strategies to engineer vascular grafts have often been directed at recreating these two specific vessel layers, typically by using (bi-layered) tubular scaffolds containing a tunica intima with highly dispersed fibers and/or a tunica media with circumferentially aligned fibers [49, 112, 180, 181]. Recently, we created biomimetic bi-layered scaffolds with a heterotypic topology by means of a unique hybrid fabrication approach that combines solution electrospinning (SES) and melt electrowriting (MEW) [182]. The heterotypic scaffold was specifically designed to direct multi-layered vSM-like cells to orient in the near-circumferential direction on the outer side, along with a confluent functional endothelium on the luminal side, both derived from human progenitor cells. However, in these statically cultured bioengineered vessels, tissue maturation and further evaluation of the endothelial functionality was hampered due to the absence of layer-specific biochemical stimuli and physiologically relevant hemodynamic loads.

The biomechanical in vivo forces, to which cells in bioengineered grafts are exposed, consist of blood flow, which imposes wall shear stress on the lumen, and blood pressure, which imposes circumferential wall strain across the vascular wall. It is well established that ECs are mechanosensitive, where shear stress stimulates the endothelium that acts as an antithrombotic barrier, to control lumen diameter, and to regulate vascular permeability [183, 184]. Similar to ECs with a mature phenotype, endothelial progenitor cells have been shown to exhibit a response to shear stress. In fact, shear stress contributes to the advancement of these cells into mature ECs [185-188]. However, in vitro studies investigating the shear- 
responsiveness of ECs (both mature and progenitor cell types) on fibrous 3D or 2D substrates are often limited to relatively short perfusion times of up to 24 hours [110, 189-194], i.e., the characteristic time period for cells to adapt to new hemodynamics (reorganization of the cytoskeleton, cellular junctions and adaption on the gene expression level) $[195,196]$. Though studies investigating EC response to shear stress of up to multiple days exist [136, 197-201], detailed analyses at later time points of EC morphology and marker expression on the protein level, especially on fibrous substrates, are lacking. To have a better indication of graft performance with respect to EC functionality and retention, a prolonged exposure to flow in a physiological 3D environment is necessary.

The performance of bioengineered grafts further depends on the communication between the shear stress-stimulated ECs in the tunica intima and the vSMCs in the tunica media. This cross-communication is necessary to regulate vasoconstriction and vasodilatation, as well as the formation and remodeling of the extracellular matrix (ECM) in response to trauma and (changing) hemodynamics, thereby maintaining a vascular homeostasis [202]. Thus, vSMCs are indispensable for the creation of functional bioengineered grafts, and should therefore be included with the required phenotype in the engineered tunica media. A challenge herein is to induce and maintain the respective phenotype when co-cultured with other progenitorderived cell types within one culture system. For example, where MSCs or vSMCs typically require TGF $\beta$ to differentiate to the contractile phenotype, ECs will undergo Endothelial-toMesenchymal-Transition (EndoMT) when exposed to TGF $\beta[203,204]$, hampering the effective cell-specific differentiation when EC and vSMC are co-cultured on vascular grafts.

In summary, the major objective for progressing our biomimetic bi-layered graft into a neovessel is the simultaneous layer-specific maturation of the cells in a physiologically relevant environment. In this work we addressed this challenge by designing and validating a perfusable, two-compartment bioreactor chamber that allows for prolonged culture (i.e., more than 24 hours) of the bi-layered vascular grafts under luminal shear stress, with a compartmentalized exposure of the involved cell types to specific culture media. We validated the approach by co-seeding human endothelial colony forming cells (ECFCs) and multipotent mesenchymal stromal cells (MSCs) in our vascular grafts and perfusing the constructs for 2 days at a physiological shear rate, with the inner and outer sides of the graft exposed to their corresponding cell-specific culture media. The established cell phenotypes of the ECs and vSMCs as well as matrix production were evaluated after perfusion.

\section{Materials and Methods}

\subsection{Bioreactor design}

A two-compartment bioreactor system was designed to be able to apply physiological shear rates at the luminal side of the vascular scaffold, while also allowing the exposure of both cell layers of the bi-layered graft (i.e., the inner and the outer compartments) to 
cell-specific media. The platform consisted of a custom-made culture chamber in which a bi-layered tubular scaffold ( $\varnothing 3 \mathrm{~mm}, 2 \mathrm{~cm}$ length) was mounted (Figure $3.1 \mathrm{~A}$ ). The luminal side of the scaffold was connected to a flow loop for unidirectional flow application, whereas the outer side of the scaffold was exposed to static culture conditions. During perfusion, flow was recorded with a ultrasonic flow meter, while temperature $\left(37^{\circ} \mathrm{C}\right), \mathrm{CO}_{2}$ level (5\%), and sterility were maintained. The flow was controlled by an Ibidi pressure pump (Ibidi $\mathrm{GmbH}$, Martinsried, Germany) by tuning the pump pressure (Figure $3.1 \mathrm{~B}$ ). Assuming Poiseuille flow, the shear stress at the luminal wall $\tau_{w}$ was computed from the flow magnitude [205]:

$$
\tau_{\mathrm{w}}=\frac{4 \mu Q}{\pi r^{3}}
$$

with $\mu$ the dynamic viscosity, $Q$ the applied flow rate, and $r$ the inner radius of the scaffold. Assuming a constant medium viscosity $\left(\mu=0.7 \mathrm{mPa} \cdot \mathrm{s}\right.$ at $\left.37^{\circ} \mathrm{C}\right)$ and using a scaffold inner radius of $1.5 \mathrm{~mm}$, the shear stress at the luminal wall (in $\mathrm{Pa}$ ) scales linearly with the applied flow rate (in $\mathrm{ml} / \mathrm{min}$ ) via: $Q=227 \tau_{\mathrm{w}}$. To apply $80 \mathrm{mPa}$ shear stress, we applied a flow rate of $\sim 18 \mathrm{ml} / \mathrm{min}$ (Supplementary Figure 3.1), which corresponds to a physiological shear rate (i.e., ${ }{ }_{\mathrm{w}} / \mu$ ) of $\dot{\gamma}=114 \mathrm{~s}^{-1}$.

A

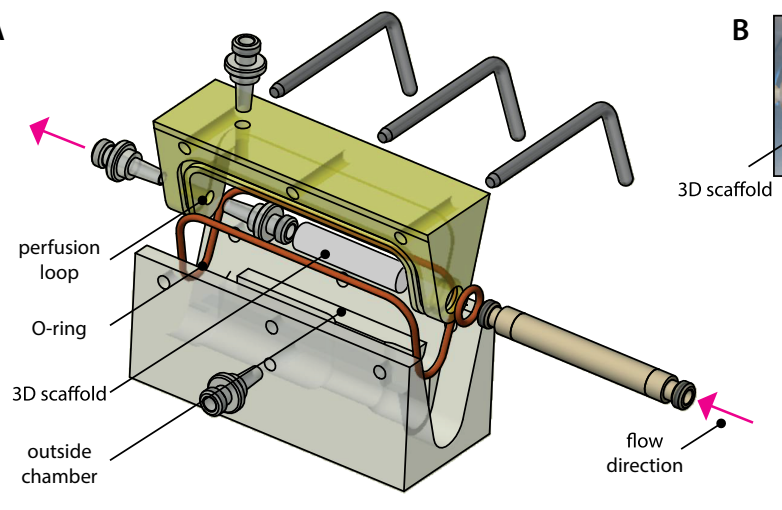

B

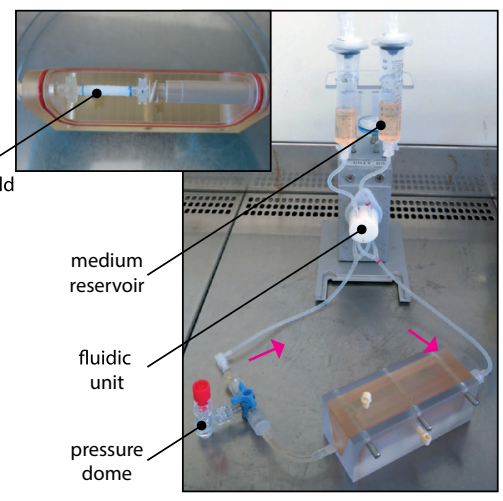

Figure 3.1. Bioreactor design. A) Exploded view of the flow chamber with the most important parts indicated. B) Photograph of the flow chamber connected to the perfusion loop (inset shows a mounted scaffold, pink arrows indicate flow direction).

\subsection{Scaffold preparation}

Bi-layered tubular scaffolds ( $\varnothing 3 \mathrm{~mm}, 2 \mathrm{~cm}$ length) were fabricated from poly( $\varepsilon$-caprolactone) (PCL) by combining SES and MEW as previously described [182]. In short, randomly oriented fiber on the luminal side were created with SES, followed by deposition of oriented MEW fibers that served as a guidance for MSCs to adhere to and grow on. To assess their 
microarchitecture, scaffolds were sputter-coated with a platinum coating ( $4 \mathrm{~nm}$, EM ACE600, Leica) and analyzed using scanning electron microscopy (SEM; Crossbeam 340, Carl Zeiss). The resulting scaffolds were sterilized by gamma irradiation, dipped in $70 \%$ ethanol, and sutured onto male luers. After suturing, the scaffolds were once again sterilized by UV exposure (15 minutes on two sides). Prior to cell seeding, the scaffolds were first washed with phosphate-buffered saline (PBS) and afterwards coated in a collagen I/fibronectin solution (30 $\mathrm{gg} / \mathrm{mL}$ rat tail collagen type I, Corning $354249 ; 50 \mu \mathrm{g} / \mathrm{mL}$ fibronectin from bovine plasma, Sigma-Aldrich F1141 in PBS) for 1 hour at $37^{\circ} \mathrm{C}$ to enhance cell attachment and retention to the scaffold. As a final step, scaffolds were incubated in complete endothelial growth medium-2 (EGM-2) containing Endothelial Basal Medium-2 + SingleQuots (Lonza), 100 U/ $\mathrm{ml}$ penicillin and $100 \mu \mathrm{g} / \mathrm{ml}$ streptomycin (PenStrep; Gibco), and 10\% heat-inactivated FBS (FBS-HI; Biowest S14068S1810) for 1 hour at $37^{\circ} \mathrm{C}$ before seeding.

\subsection{Cell culture and characterization}

\subsubsection{Endothelial colony forming cells}

ECFCs were isolated and characterized as described previously [206]. In short, ECFCs were isolated from the cord blood of two different donors (the procedure was approved by the medical research ethics committee, University Medical Center Utrecht; informed consent was obtained from the mothers). The isolates are hereafter referred to as ECFC1 and ECFC2. Confirmation of their potency was performed by phenotypic characterization with flow cytometry as described and presented previously [36]. After isolation, the ECFCs were either frozen or further expanded in complete EGM-2 medium. The cells were used for scaffold seeding at passage 7 or 9 (ECFC1) and 9 or 10 (ECFC2).

\subsubsection{Multipotent mesenchymal stromal cells}

MSCs were isolated and characterized as described previously [206]. In short, MSCs from human bone marrow aspirates were obtained from consenting patients $(n=2$; the procedure was approved by the local medical research ethics committee, University Medical Center Utrecht). These isolates are referred to as MSC1 and MSC2. The multipotency of the MSCs was confirmed via differentiation towards adipogenic, osteogenic, and chondrogenic lineages, as well as via phenotypical characterization by cell surface marker expression profiles with flow cytometry as described elsewhere $[103,206]$. MSCs with confirmed multipotency were further expanded in MSC expansion medium, consisting of a-Minimum Essential Medium (Gibco Paisley, 22561), supplemented with 10\% FBS-HI, $100 \mathrm{U} / \mathrm{ml}-100$ mg/ml PenStrep, 0.2 $\mathrm{mM} \mathrm{L}$-ascorbic acid-2-phosphate (ASAP, Sigma), and $1 \mathrm{ng} / \mathrm{ml}$ basic fibroblast growth factor (rh-FGF-2; R\&D Systems). The MSCs were used for scaffold seeding at passage 4.

\subsubsection{Cell seeding}

Prior to seeding, the medium was removed from the scaffolds and a suspension (100 $\mu \mathrm{l})$ of complete EGM-2 medium containing 3.8 million ECFCs/ml was carefully injected into the 
lumen using a syringe with a $25 \mathrm{G}$ blunted needle. To promote uniform cell attachment, the cell-loaded constructs were rotated by $90^{\circ}$ every 15 minutes for $1 \mathrm{~h}$ at $37^{\circ} \mathrm{C}$. Subsequently, excess medium was removed from the lumen and a total volume of $80 \mu \mathrm{lEGM}-2$ medium containing 3.8 million MSCs/ml was carefully pipetted onto the outer scaffold layer. The MSCs were allowed to adhere for $1 \mathrm{~h}$ at $37^{\circ} \mathrm{C}$, during which the constructs were rotated by $180^{\circ}$ after $30 \mathrm{~min}$. After cell seeding, the constructs were cultured on a continuous rotating orbital shaker $(50 \mathrm{rpm})$ at $37{ }^{\circ} \mathrm{C}$ and $5 \% \mathrm{CO}_{2}$ for 7 days in petri dishes $(\varnothing 60 \mathrm{~mm}$ ) containing $10 \mathrm{ml}$ complete EGM-2 medium, which was refreshed at day 3 (Figure 3.2).

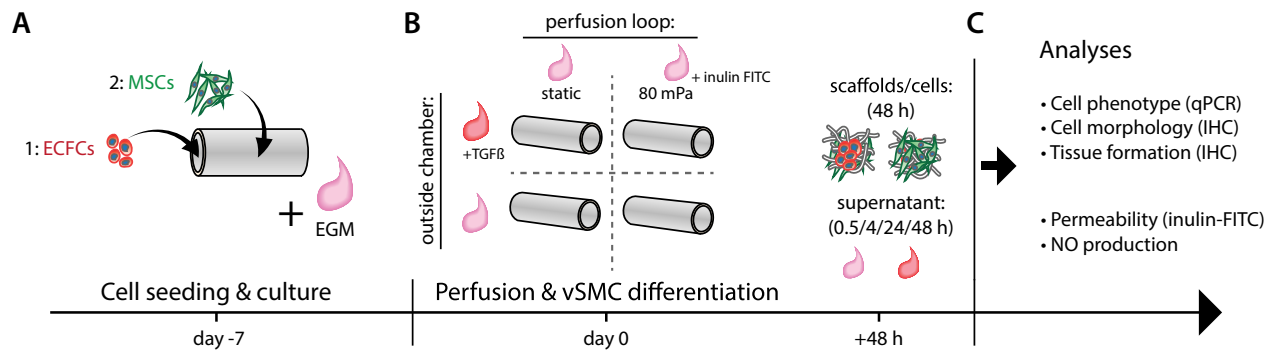

Figure 3.2. Experimental outline detailing cell seeding, differentiation and analyses. A) Endothelial colony forming cells (ECFCs) and multipotent mesenchymal stromal cells (MSCs) were seeded on the luminal and outer sides of a 3D tubular scaffold and kept in culture for 7 days. B) After bioreactor mounting, the constructs were exposed to luminal flow, TGF $\beta$ stimulation in the outside chamber, or a combination thereof for $48 \mathrm{~h}$. C) After the perfusion period, constructs and supernatants were collected and processed for further analyses.

\subsubsection{Perfusion and vSMC differentiation}

After the 7-day pre-perfusion period, the constructs were mounted into the bioreactor and connected to the flow loop to start the 2 -day perfusion period. The constructs were divided in 4 groups: perfusion on the luminal side with complete EGM-2, together with vSMC differentiation medium supplied to the outer compartment ( $\mathrm{p} / \mathrm{tgf})$; perfusion, and complete EGM-2 medium in the outer compartment (p/egm); static culture (no perfusion), and vSMC differentiation medium in the outer compartment (s/tgf); and static culture with complete EGM-2 medium supplied to the outer compartment (s/egm) (Figure 3.2 B). For groups p/tgf and p/egm, the flow-loop was filled with $13 \mathrm{ml}$ of complete EGM-2 medium supplemented with $0.1 \mathrm{mg} / \mathrm{ml}$ Fluorescein isothiocyanate-inulin (FITC, $3 \mathrm{kDa}$ ) (TdB Consultancy) in $0.1 \%$ DMSO for determining the permeability of the cultured scaffold (Section 3.4.2). To increase the flow in a stepwise fashion to $80 \mathrm{mPa}$ (with a shear rate of $\sim 100 \mathrm{~s}^{-1}$ ), the pump pressure was increased from 3 mbar to 25 mbar within a period of 12 hours (with intermediate steps at 5 and $8 \mathrm{mbar}$ ), and maintained at 25 mbar for the following 36 hours (Supplementary Figure 3.1). The static groups s/tgf and s/egm were not exposed to this flow protocol, but were only shortly flushed with complete EGM-2 medium without FITC-inulin once an hour (at $5 \mathrm{mbar}$ for $60 \mathrm{~s}$ ) to refresh the culture medium in the lumen. For groups $\mathrm{p} / \mathrm{tgf}$ and s/tgf, 
the outside of the culture chamber was filled with $13 \mathrm{~mL}$ of vSMC differentiation medium (DMEM high glucose (Gibco Paisley, 31966), 10 \% FBS-HI, 100 U/ml-100 $\mu \mathrm{g} / \mathrm{ml}$ PenStrep, 30 $\mu \mathrm{M}$ ASAP, and $5 \mathrm{ng} / \mathrm{mL}$ TGF $\beta 1$ (PeproTech 100-21C)) for induction of vSMC differentiation. The groups $\mathrm{p} / \mathrm{egm}$ and s/egm were cultured with $13 \mathrm{ml}$ of complete EGM-2 medium in the outer chamber. All experiments were performed with two donor combinations ( $n=4$ per donor combination in the flow conditions, and $n=2$ in de static conditions).

\subsection{Analyses}

\subsubsection{Sample processing}

After the 2-day perfusion period, the constructs were collected, partitioned as illustrated in Supplementary Figure 3.2, and processed for further analysis (Figure 3.2 C). Sample pieces designated for immunohistochemistry and whole mount fluorescence staining were stored at $4{ }^{\circ} \mathrm{C}$ after 60 min fixation in $3.7 \%$ formaldehyde and $3 \times 5$ min washing in PBS. Samples for total cellular RNA extraction were resuspended in $0.5 \mathrm{ml}$ Trizol Reagent (Thermo Fischer Scientific) for 5 minutes at room temperature and subsequently stored at $-80^{\circ} \mathrm{C}$ till further use. The constructs were processed for assessment of cell morphology and characterization of phenotype (stainings and $\mathrm{qPCR}$ ), and to investigate tissue formation (stainings). Additionally, medium samples were collected from the flow loop during perfusion $(200 \mu \mathrm{l}$ at $30 \mathrm{~min}, 4 \mathrm{~h}, 24 \mathrm{~h}$, and $48 \mathrm{~h}$ ) and from the outer chamber after perfusion (at $48 \mathrm{~h}$ ) and stored at $-20^{\circ} \mathrm{C}$ until further analysis to determine the construct permeability.

\subsubsection{Permeability}

To monitor the exchange of media between the two compartments of the culture chamber, and thus the permeability of the constructs, FITC-inulin was supplemented to the flow loop prior to the perfusion period (Figure 3.3 A). To evaluate the influence of the cell coverage on this permeability, the results of the cell-seeded constructs were compared against unseeded scaffolds (i.e.,'empty scaffolds'). Medium ( $200 \mu \mathrm{l}$ ) from the empty scaffolds was collected after $30 \mathrm{~min}, 90 \mathrm{~min}, 4 \mathrm{~h}$, and $48 \mathrm{~h}$ of perfusion. Since FITC-inulin is a relatively small fluorescent molecule $(3 \mathrm{kDa})$, of which the fluorescence scales linearly with its concentration, it can be used to quantify the permeability of the construct to inulin). Fluorescence was detected at an emission wavelength of $535 \mathrm{~nm}$ in the collected medium samples after excitation at 485 $\mathrm{nm}$ while using a microplate reader (Synergy HTX; Biotek).

\subsubsection{Gene expression}

RNA isolation.

Samples were mixed with $20 \%$ Chloroform (Millipore) for 3 minutes at room temperature and subsequently centrifuged at $12,000 \mathrm{G}$ for 15 minutes $\left(4^{\circ} \mathrm{C}\right)$. The colorless upper aqueous phase was mixed with the same amount of 2-propanol (Sigma) ( 0.25 ml) and $0.5 \mu \mathrm{l}$ glycogen (R0551, Thermo Fisher Scientific). The mixture was then left at room temperature for $10 \mathrm{~min}$ and centrifuged at $12,000 \mathrm{G}$ for $10 \mathrm{~min}\left(4^{\circ} \mathrm{C}\right)$. The resulting pellet 
was washed in $75 \%$ ethanol and again centrifuged at $7,500 \mathrm{G}$ for $5 \min \left(4^{\circ} \mathrm{C}\right)$. After drying, the pellet was re-suspended in $10 \mu$ l of RNase-free water and exposed to DNAse treatment ( $1 \mu \mathrm{l}$ of Turbo DNase buffer (AM2238, Ambion) and $0.5 \mu \mathrm{l}$ DNase $(2 \mathrm{U} / \mu \mathrm{l})$ ).

Quantitative polymerase chain reaction (qPCR).

Total RNA was reverse-transcribed into cDNA with an iScript cDNA synthesis kit (Biorad, 1708890). The RNA input concentration was set to $5 \mathrm{ng} / \mu \mathrm{l}$ for all samples, providing the same CDNA end concentration for all samples used in this data set. FastStart SYBR green master (Sigma, 04673484001) was used for the qPCR reaction mix, with a total end volume of $10 \mu \mathrm{l}$ containing $10 \mathrm{ng}$ of CDNA and $500 \mathrm{nM}$ primer (forward and reversed). Glyceraldehyde-3phosphate dehydrogenase (GAPDH) was chosen as optimal housekeeping gene among a set of candidates, based on its expression stability tested with the algorithm from Normfinder. Primers were optimized for their amplification efficiency (calculated via: $E=-1+10^{-1 / \text { slope }}$ ). Primer sequences and annealing temperatures can be found in Supplementary Table 1. Gene expression was evaluated for 3 main marker outcomes: VSMC associated markers (a smooth muscle actin (ACTA2/aSMA), smooth muscle protein 22 a (transgelin/SM22a), Calponin (CNN1/CALP), and (tropo)elastin (ELN)), EC associated markers (PECAM1/CD31, vascular endothelial-cadherin (CDH5/NE-cadherin), and shear responsive markers (Cyclo-oxygenase-2 (PTGS2/COX-2), Endothelial Nitric Oxide Synthase (NOS3/ eNOS), and Krüppel-like Factor 2 (KLF2)).

Relative gene expression analysis.

Cyclic threshold $(\mathrm{Ct})$ values were normalized for the housekeeping gene and relative fold gene expression was calculated according to the $2^{-\Delta A C T}$ method. To test for VSMC induction, the relative gene expression ( $2^{-\triangle C T}$ ) of the TGF $\beta$-stimulated samples ( $p / \operatorname{tgf}$ and $\mathrm{s} / \mathrm{tgf}$ ) was normalized to their matched controls (i.e., cultures from same donor for $\mathrm{p} /$ egm and s/egm). To test for the influence of shear stress on the endothelium, $2^{-\Delta C T}$ of the perfused samples was normalized to their matched controls (i.e., static samples). Finally, to confirm that the differentiation medium in the outer compartment did not influence the endothelium, $2^{-\Delta C T}$ of the perfused, TGF $\beta$-stimulated samples ( $\left.p / t g f\right)$ were normalized to the perfused, undifferentiated controls ( $\mathrm{p} / \mathrm{egm}$ ). For statistical analysis, the $2^{-\mathrm{AcT}}$ of the matched controls was normalized to its own group average.

\subsubsection{Stainings}

Whole mount staining and stained sections ( $5 \mu \mathrm{m}$ paraffin sections) were analyzed to assess cell phenotypes and matrix composition.

Immunocytochemistry.

Formalin-fixed whole mount samples were permeabilized in $0.5 \%$ Triton-X 100 in PBS. Non-specific binding was blocked for 30 minutes using $5 \%(\mathrm{w} / \mathrm{v})$ bovine serum in PBS. 
Primary antibodies were incubated overnight at $4{ }^{\circ} \mathrm{C}$ or for 1 hour at room temperature (Supplementary Table 2). After washing in PBS ( $3 \times 5$ minutes), fluorescently labeled secondary antibodies were added overnight at $4{ }^{\circ} \mathrm{C}$ or for 1 hour at room temperature with possible addition of phalloidin (FITC or TRITC, Supplementary Table 2). In case of CD31 or calponin identification, the $A B C$ method was used for amplification of the signal with secondary biotinylated antibodies, detected with fluorescently labelled streptavidinconjugated tertiary antibodies. Nuclei were stained with 4',6-diamidino-2-phenylindole (DAPI, $100 \mathrm{ng} / \mathrm{ml}$ ) for 15 minutes. Whole mount constructs were kept in PBS and visualized by confocal laser scanning (SP8X Leica DMi8 with a 10x/0.4 APO lens, 20x/0.4 fluotar lens, or $63 \mathrm{x} / 1.40$ APO CS2 lens).

Immunohistochemistry.

Deparaffinized and rehydrated sections were permeabilized in $0.5 \%$ Triton-X 100 in PBS. Depending on the antibody, antigen retrieval was performed by incubation in trypsin (30 minutes $37^{\circ} \mathrm{C}$ for laminin a5 subunit), pepsin ( 12 minutes $37^{\circ} \mathrm{C}$ for collagen III), or citrate (20 minutes $97^{\circ} \mathrm{C}$ for collagen I). Non-specific binding was blocked for 30 minutes using 5 $\%(\mathrm{w} / \mathrm{v})$ bovine serum in PBS. Primary antibodies were incubated overnight at $4{ }^{\circ} \mathrm{C}$ or for $1 \mathrm{~h}$ at room temperature (Supplementary Table 2). After washing in PBS ( $3 \times 5$ minutes), fluorescently labeled secondary antibodies were added overnight at $4{ }^{\circ} \mathrm{C}$ or for 1 hour at room temperature, together with DAPI (Supplementary Table 2). In case of staining for laminin a5 subunit, goat anti-mouse conjugated HRP-labeled polymer was added for 1 hour (Dako, Envision, K400011). Addition of DAB substrate solution resulted in a brown staining, facilitated by HRP. Nuclei were counterstained with Mayer's hematoxylin (Merck). Finally, the sections were dehydrated, mounted with DEPEX, and visualized with a bright field microscope (Leica BX51). The fluorescently labeled sections were mounted in mowiol and visualized with an inverted fluorescence microscope (Leica DMi8, 1 pixel $=0.65 \mu \mathrm{m}$ or $0.32 \mu \mathrm{m}$ with a $20 \times / 0.4$ or $40 \times / 0.95$ HC PL Fluotar lens, respectively).

Histochemistry.

Deparaffinized sections were stained with routine hematoxylin and eosin (H\&E) or with Weigert's hematoxylin (10 minutes). Collagenous structures in hematoxylin-stained sections were visualized with PicroSirius red stain ( 1 hour in $0.1 \mathrm{~g}$ Sirius red (F3B Klinipath No.80115) in $100 \mathrm{ml}$ saturated aqueous picric acid (Riedel-deHaën No.36011) and 2 minutes in $0.01 \mathrm{M} \mathrm{HCl}$ ). After dehydration, samples were mounted in DEPEX and imaged with a bright field microscope (Leica BX51). Collagenous structures were also imaged under polarized light.

\subsubsection{Cellular orientation}

The in-plane orientation of the cells was quantified from the F-actin images using inhouse developed software as described elsewhere [207]. In short, the actin fiber angle 
distribution in the constructs was calculated by binning the principal direction in each pixel of the F-actin images into a histogram ( $n_{\text {bin }}=45$ between 0 rad and $\pi \mathrm{rad}$ ). The fiber distributions were assessed for alignment induced by flow (ECs) and by the contact guiding cue of the MEW fibers (vSMCs). Cellular orientation of the ECs was quantified from maximum intensity confocal projections at 6 locations per sample. For the vSMCs, 3 different z-stacks $(\sim 350 \mu \mathrm{m})$ per sample were used for the orientation analysis.

\subsection{Statistics}

Statistical analyses were performed with GraphPad Prism 6.01. Permeability measurements were tested for the influence of cell coverage on the maintenance of an inulin concentration difference between the inner and outer compartment. Gene expression outcomes were tested for the influence of VSMC phenotype induction on the SMCs, shear stress on the ECs, and VSMC differentiation medium on the endothelium. The inulin concentration differences and fold changes of each gene of interest were tested for significant differences using a student's t-test or a non-parametric Mann-Whitney U test, depending on the normality of the groups (checked with the Pearson normality test). Differences between the experimental groups were considered statistically significant when $p<0.05$.

\section{Results}

\subsection{Cell-laden scaffolds form a leak-free construct}

PCL was processed into bi-layered tubular scaffolds with an inner diameter of $3 \mathrm{~mm}$ and wall thickness of $\sim 250 \mu \mathrm{m}$ (Figure $3.3 \mathrm{~B}$, left panel). The inner layer, with a thickness of $\sim 50 \mu \mathrm{m}$, exhibited an isotropic microstructure with fiber diameter of $1.4 \pm 0.2 \mu \mathrm{m}$. The outer layer contained stacked fibers of $15.2 \pm 4.8 \mu \mathrm{m}$ diameter with two predominant orientations ( $71.6 \pm 0.6^{\circ}$ with respect to the longitudinal axis). Next, the capacity of the cells and matrix to seal the scaffold was assessed, which is necessary to retain the cell-specific media in the respective compartments (Figure 3.3 A). SEM images indicated that the cells and matrix completely covered the inner and outer layer (Figure 3.3 B, right panel). FITCinulin diffusion measurements revealed that the concentration of inulin in the cell-laden constructs approached a plateau between $4 \mathrm{~h}$ and $24 \mathrm{~h}$ of perfusion, which significantly differed from the concentration in the outer compartment after $48 \mathrm{~h}(\mathrm{p}=0.004)$. In contrast, the media from the inner and outer compartments of the empty scaffolds were completely mixed after $48 \mathrm{~h}$ (Figure 3.3 C-D). The limited diffusion between the inner and outer chamber in the cell-laden constructs validated our approach to separately provide cell-specific media to each cell type. 
A

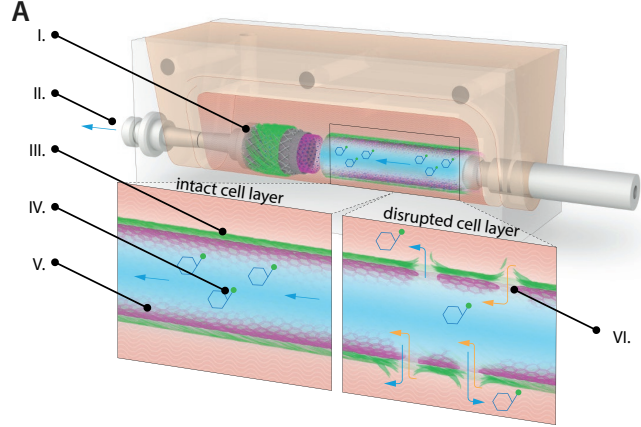

C

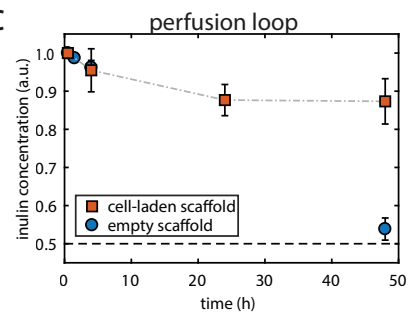

B



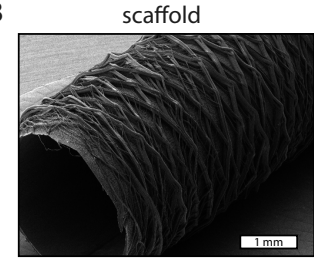

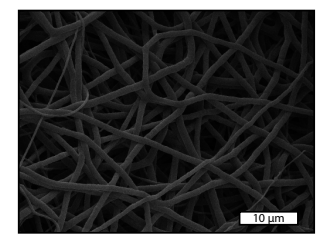

scaffold

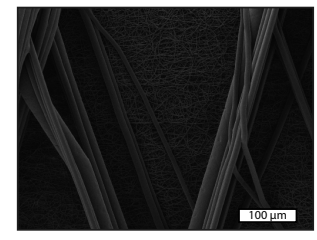

cell-laden
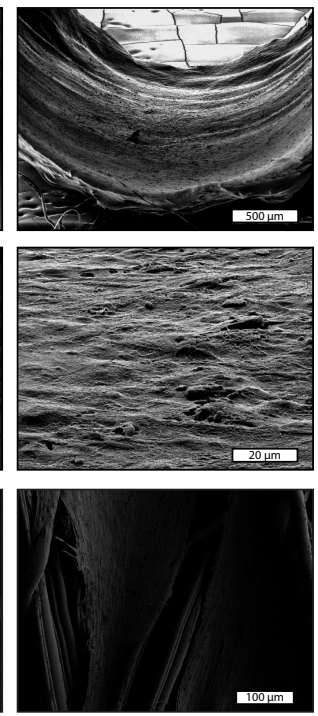

Figure 3.3. Characterization of the scaffold and cell-laden construct. A) Schematic illustration of the complete setup and the diffusion of soluble factors between the inner and outer compartments (I. bi-layered graft, II. flow direction, III. vSMC layer, IV. inulin-FITC, V. EC layer, VI. diffusion between perfusion loop and outer compartment). B) Scanning electron microscopy images without (left column) and with cells (right column) at a small magnification (top), and at the inner (center) and outer side (bottom) of the scaffold. C) The normalized inulin concentration in the perfusion loop during the time course of the experiment using cell-laden scaffolds (red squares) and with empty scaffolds (blue circles), demonstrating that the addition of cells resulted in a leak-free construct (a.u.; arbitrary unit). D) After 48 hours of perfusion, there is a significant difference $(p=0.004)$ in the inulin concentration between the perfusion loop (inside) and the outside chamber (outside) in cell-laden scaffolds (red squares), but not in empty scaffolds (blue circles). Complete mixing of both compartments at a normalized concentration of 0.5 is indicated by the dashed lines in $\mathbf{C}$ ) and $\mathbf{D}$ ).

\subsection{The endothelial layer on the luminal side is responsive to shear stress}

The SES layer on the luminal side provided a substrate for the ECs to form a confluent endothelium on, with CD31 expression localized at the cell adherens junctions (Figure 3.4 A). The cytoskeletal F-actin in the static groups was mainly confined to the cell periphery in the absence of shear stress, but remodeled to thick dorsal stress fibers under shear stress. Along with the endothelium, a collagen IV-positive matrix was present, known to be one of the main components of the basement membrane (Figure 3.4 A). The endothelial cells elongated under perfusion, as expected due to the flow-responsiveness of endothelium (Figure 3.4 A). The endothelium integrity was retained over the complete length of the scaffold, demonstrating the endothelium's robustness to withstand the flow, and the cells appeared in a 'wave-like' organization without preferred global orientation in the direction of flow (Figure 3.4 B). Quantification of the F-actin distribution confirmed this (Figure $3.4 \mathrm{C}$ ). After perfusion, the monolayer still showed CD31 localization at the cell 
borders as well as VE-cadherin-positive adherens junctions (Figure 3.4 D), indicating that it preserved its selective barrier function. Localization of vinculin confirmed the linkage between the actin cytoskeleton and the ECM via focal adhesion complexes (Figure 3.4 E and Supplementary Figure 3.3 B).

The responsiveness of the endothelium to flow was also observed at the gene expression level. Perfused endothelium showed significant upregulation of the shear stressassociated enzymes eNOS, COX-2 and KLF2, compared to static endothelium (Figure 3.4 F). Furthermore, the markers CD31 and VE-cadherin, known for their role in shear responsive pathways and permeability function, were found to be upregulated after flow perfusion. Shear stress did not have a clear effect on the expression of the platelet adhesion molecule vWF on a gene expression level $(p=0.2323)$, but a conformational change of the VWF protein under shear stress was observed whereby the unfolded active form of the glycoprotein appeared in strand-like multimers (Figure 3.4 D), compared to a globular appearance in static control cultures (Supplementary Figure 3.3 A). Presence of TGF $\beta$ in the differentiation medium of the outer compartment did not affect the appearance and gene expression of the endothelium, in either the static or perfused condition, indicating that the sealing capacity of the construct prevented TGF $\beta$ to diffuse to the endothelial compartment (Supplementary Figure 3.3 C-D). 

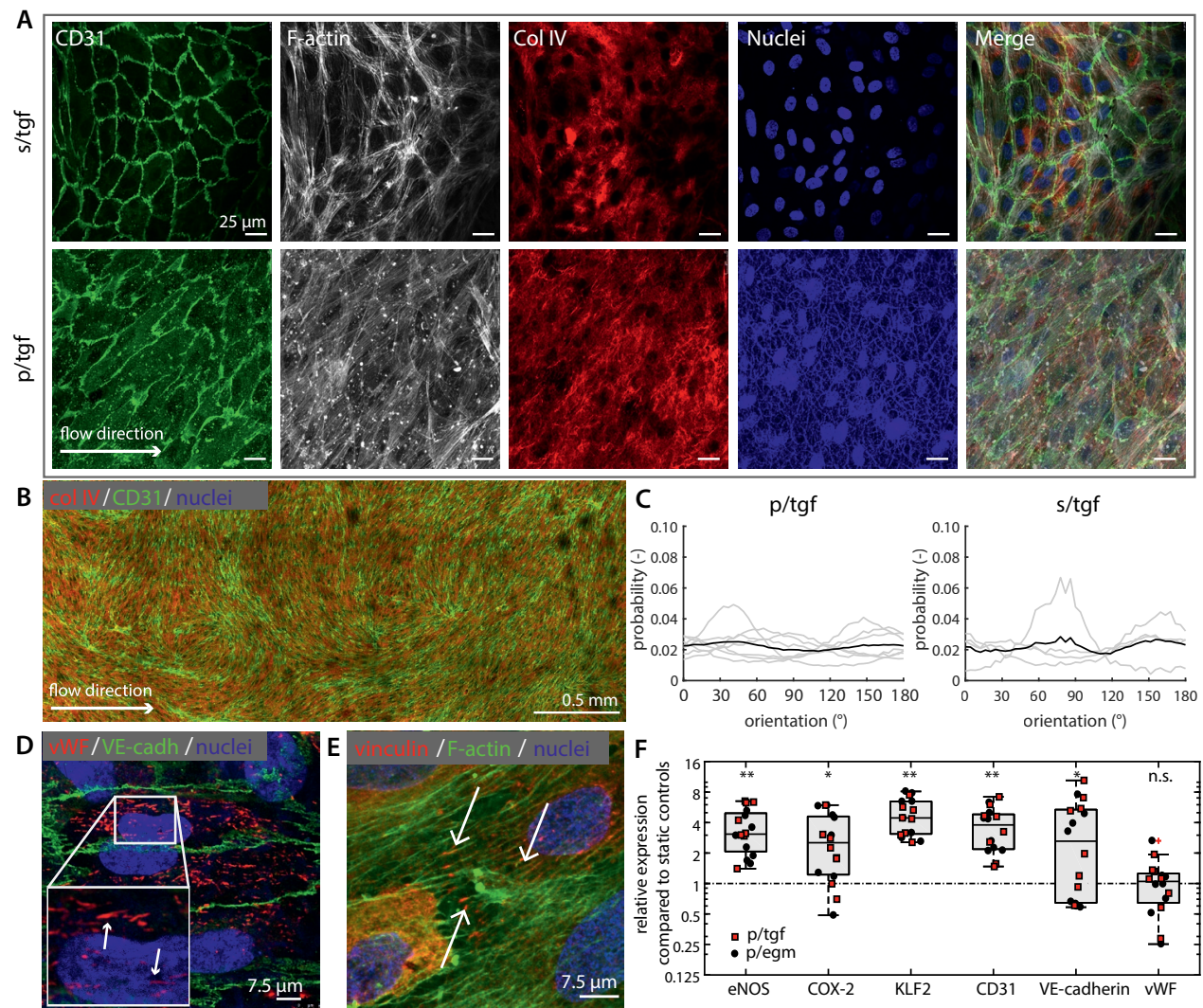

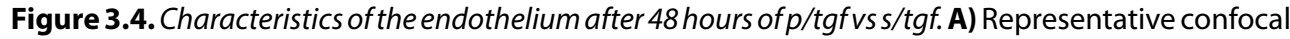
visualizations of the endothelial cells (ECFCs) cultured under static (top row) and flow (bottom row) conditions, while containing vSMC differentiation medium in the outer chamber (s/tgf and p/tgf). B) Overview image showing cell coverage and orientation over the full length of the perfused sample. C) Histograms quantifying EC F-actin angular distribution under flow (left, p/tgf) and static (right, $\mathrm{s} / \mathrm{tgf}$ ) conditions show no preferred cellular orientation (each grey line represents an individual sample, the black line represents the average; flow direction at $0^{\circ} / 180^{\circ}$ ). D) Localization of the shearinduced strand-like morphology of von Willebrand Factor as well as close cell-cell contacts between ECs shown by VE-cadherin. E) Focal adhesion complex component vinculin (arrows) in the luminal cell layer co-localized with F-actin filaments. F) Relative gene expression after 48 hours of perfusion ( $\mathrm{p} / \mathrm{tgf}$ and $\mathrm{p} / \mathrm{egm}$ ) compared to their matched controls (i.e., static controls in TGFß-stimulated samples ( $\mathrm{s} / \mathrm{tgf}$, red squares) and EGM controls (s/egm, black dots), respectively) show upregulation of EC and shear stress-associated genes (n.s.: not significant, ${ }^{*} p<0.01$, ${ }^{* *} p<0.0001$, boxplots contain the combined data of the $\mathrm{p} / \mathrm{tgf}$ and $\mathrm{p} / \mathrm{egm}$ groups).

\subsection{MSCs differentiate to vSM-like cells}

To evaluate if the addition of TGF $\beta$ to the outer compartment resulted in differentiation of MSCs into vSM-like cells, the contractile vSMC markers calponin and aSMA were examined. Indeed, differentiation was confirmed by the appearance of calponin ${ }^{+} / \mathrm{aSMA}^{+}$cells (Figure 3.5 A and Supplementary Figure 3.4) and by the significant upregulation of these markers 
at the gene expression level (Figure 3.5 B). No significant difference was found in the expression of the SM22a gene $(p=0.117)$. After differentiation, the matrix surrounding the vSM-like cells contained the required vSMC basement membrane component collagen IV (Figure $3.5 \mathrm{E}$ ), and (tropo)elastin also showed significant upregulation on the mRNA level (Figure $3.5 \mathrm{~B}$ ). In addition to the expected marker expression of the cells, the cells also elongated and aligned in the circumferential orientation (Figure 3.5 C-D), guided by the MEW fibers.

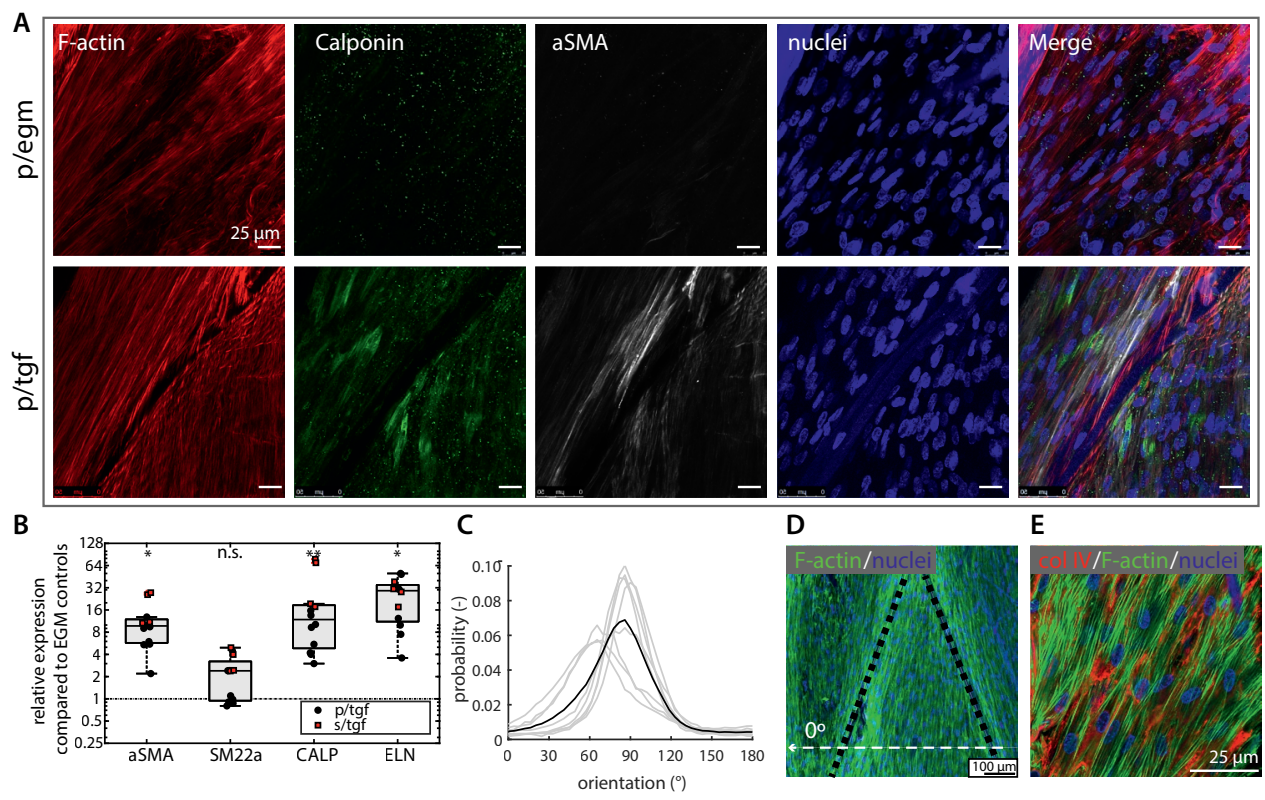

Figure 3.5. Vascular smooth muscle cell characterization for $p / t g f v s$ p/egm after 48 hours of perfusion. A) Representative confocal visualization of the smooth muscle-like cells cultured without (top row) and with (bottom row) vSMC differentiation medium. B) Relative gene expression after 48 hours of TGF $\beta$ stimulation compared to EGM controls in perfused (black dots) and static (red squares) samples, showing upregulation of vSMC associated markers (n.s.; not significant, ${ }^{*} p<0.001$, ** $\mathrm{p}<0.0001$, boxplots contain the combined data of the $\mathrm{p} / \mathrm{tgf}$ and $\mathrm{s} / \mathrm{tgf}$ groups). C) Histograms quantifying F-actin angular distribution in TGF $\beta$-stimulated samples ( $\mathrm{s} / \mathrm{tgf}$ and $\mathrm{p} / \mathrm{tgf}$ ), showing a preferentially circumferential orientation (each grey line represents the average of an individual sample, the black line represents the group average; long axis along $0-180^{\circ}$ ). D) Overview image of the F-actin organization (MEW fiber orientation indicated by black dotted line, axial direction indicated by horizontal white dotted line). E) Collagen IV-positive matrix surrounding the cells.

\subsection{Co-cultured bi-layered grafts resemble the native vessel structure}

Finally, the effects of the differentiation medium in the outer compartment on de novo tissue formation by the vSM-like cells were studied. The cells adopted an elongated morphology and organized into a multi-layered fashion around the bi-layered scaffold (Figure 3.6 A). Most strikingly, several matrix components in the TGF $\beta$-stimulated constructs were more 
abundantly expressed compared to in the EGM-cultured controls (Figure 3.6 B). Specifically, the TGF $\beta$-stimulated constructs showed a laminin a5-positive matrix with thicker collagen bundles composed of collagen type III, but mainly collagen type I. This was the other way around in the non-differentiated control samples, where more collagen type III was present than collagen type I. The cross-sectional view shows the presence of a CD31-positive continuous monolayer together with a multi-layered aSMA-positive tunica media mimic, confirming that the correct phenotype, morphology, and organization of both cell types could be induced simultaneously within one co-cultured bi-layered graft (Figure 3.6 C). Furthermore, the low MEW fiber density resulted in large pores, allowing for close cell-cell contact of the vSM-like cells surrounded by their own ECM. The cross-section demonstrates that the SES layer separated the cell types from each other, important for prevention of intimal hyperplasia by vSMC ingrowth into the luminal side.
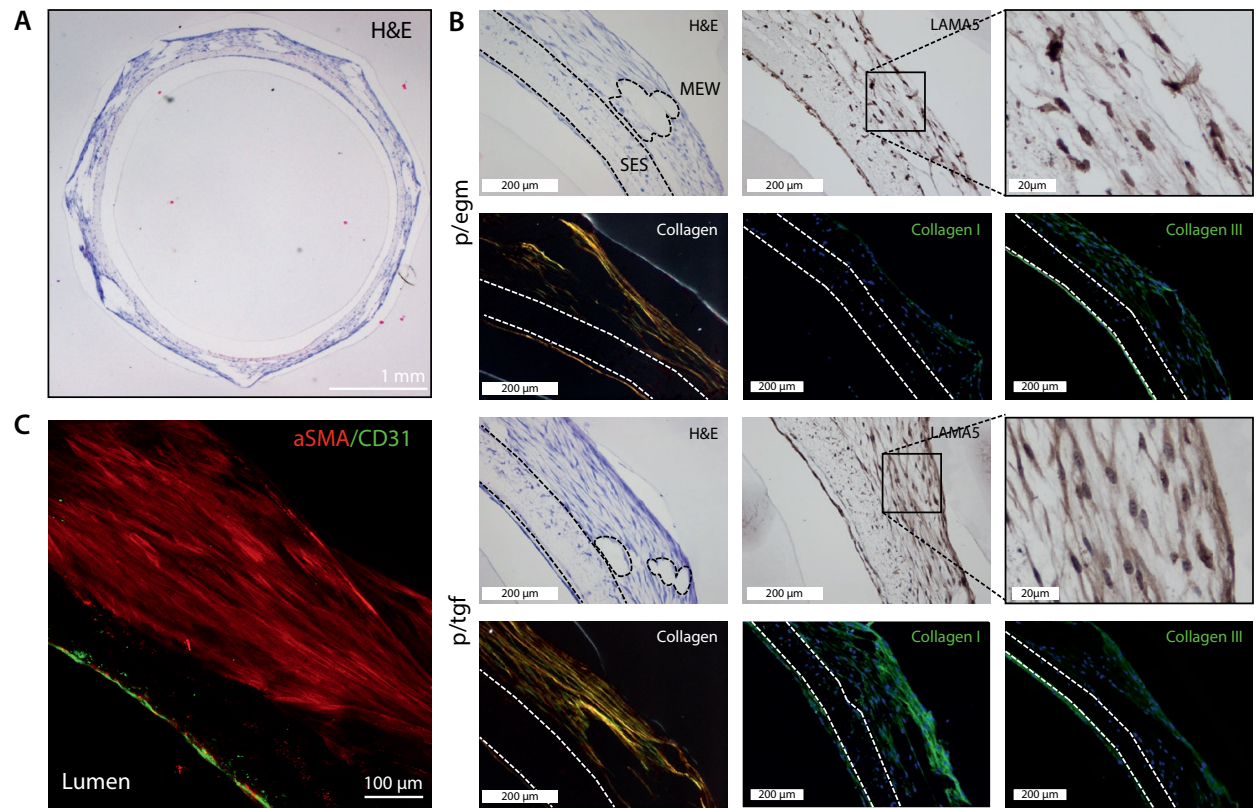

Figure 3.6. Tissue formation after 7 days of pre-culture and 48 hours of perfusion. A) Cross-sectional overview of the graft with a completely populated tunica media (hematoxylin and eosin (H\&E) staining). B) Cross-sections of representative samples cultured with $\mathrm{p} / \mathrm{egm}$ (top rows) or $\mathrm{p} / \mathrm{tgf}$ (bottom rows) indicate multiple layers of elongated vSM-like cells (H\&E). The TGF $\beta$-stimulated samples show laminin a5 expression (LAMA5) in the ECM, whereas control samples show only laminin a5 expression in the cells. Picrosirius red staining shows collagen bundles (under polarized light), further specified with stainings for collagen type I and collagen type III (dashed lines indicate the scaffold borders, SES; solution electrospinning, MEW; melt electrowriting). C) An aSMA/CD31stained cross-section shows the localization and the different phenotypes of the two cell layers within one construct. 


\section{Discussion}

We designed and validated a two-compartment bioreactor system for further maturation of our recently bioengineered vascular grafts, with maintenance of vSM-like cells and progenitor-derived ECs via compartmentalized exposure to their respective media. MSCs and ECFCs were selected as cell sources, with MSCs having the ability to differentiate into vSMCs and the ECFCs known for their inherent vasculogenic capacity and participation in post-natal vasculogenesis. Both cell types can be isolated from autologous sources, and therefore have therapeutic potential. We demonstrated that during the period of perfusion, the cells together with their deposited matrix seal off the open, porous scaffold, resulting in the retention of cell-specific differentiation media in the lumen and outer compartment. Moreover, the vascular integrity and the endothelium's attachment to the substrate were preserved throughout the period of perfusion. Using bi-layered biomimetic scaffolds, we successfully recapitulated the architecture of native vessels, consisting of circumferentially oriented, multi-layered structures of vSM-like cells and a flow-responsive confluent luminal layer of ECs.

In our set-up, the ECs were exposed to increasing levels of fluid shear stress up to $80 \mathrm{mPa}$ (at a physiological shear rate of $\sim 100 \mathrm{~s}^{-1}$ ). This level of shear stress is slightly lower than that found in veins (0.1-0.6 $\mathrm{Pa}$ [208]), but could be increased to match arterial shear levels (1-7 Pa [208]) through the addition of medium thickening components [209], and/or application of higher flow rates if desired. However, since freshly seeded ECs initially form a weak adhesion to the graft material, they would detach from the graft when directly exposed to in vivo levels of high shear stress. Here, using a gradually increasing flow protocol after static pre-culturing, complete endothelial retention was achieved during an extended period of perfusion. In our pressure-driven bioreactor system, the flow protocol can be customized for other applications as well in terms of flow rate, pulsatility, pressure, and duration, and can be monitored during the experiment.

The integrity of the monolayer was also maintained, with localization of VE-cadherin in the adherens junctions and CD31 expression in the cell periphery (Figure 3.4). Both VE-cadherin and CD31 play an important role in the vascular permeability barrier and stabilization of cell-cell junctions [210]. They also play a role in shear stress response via the formation of a multimolecular complex, together with $\beta$-catenin and VEGFR3, that is mechanoresponsive [210-212]. This mechanosensory complex is involved in a number of well-known shear stress responses by ECs (as well as their progenitor cells [188]), such as stress fiber remodeling and cell alignment [192]. Consistent with reports by others [192, 213], the ECs appeared more elongated under perfusion while their F-actin remodeled from cortical stress fibers located at the cell periphery to dorsal stress fibers throughout the cytoplasm. However, we did not observe a predominant global cell alignment, which could indicate either that a certain threshold of shear stress was not reached with the 
applied flow protocol [214], that re-orientation was hindered by the high density of the cells [215], or that adhesion of the monolayer to the underlying basement membrane was not sufficiently strong [216].

Indeed, we used a high cell seeding density to ensure the formation of a tightly connected network of ECs. However, it has been previously shown that over-confluence can impede the alignment of cells in the monolayer in the direction of flow within the observation period of 48 hours [215]. Regulation of cell morphology and F-actin organization in response to shear stress has been additionally shown to require a firm anchorage to the basement membrane via focal-adhesion-associated proteins, such as vinculin [217, 218]. In our endothelium, vinculin was mainly located at the basal side, linking the F-actin stress fibers to the underlying ECM, but without obvious re-localization towards the 'upstream' end of the cells. The localization of this focal adhesion component determines the direction of stress fiber formation and could therefore be related to the elongated morphology of the shear-stress-stimulated cells [217]. It will be interesting to investigate the relation between vinculin, focal adhesions complexes, and morphological changes (i.e., cellular elongation and re-orientation) under the influence of shear stress using more in-depth mechanobiological analyses in the future.

Responsiveness to perfusion by the endothelium was also confirmed at the gene and protein expression levels, hinting at the biological functionality of the endothelium. The anti-thrombotic function of the endothelium is partly regulated by the glycoprotein vWF, which is the main responder to vascular injury by initiating coagulation via platelet adhesion. It has been shown that VWF is responsive to shear stress, resulting in a conformational change from the inactive globular conformation into unfolded multimeric streaks [219-221]. This activation of vWF was also observed in this study, with the appearance of streaks of VWF in the perfused constructs (Figure 3.4 D and Supplementary Figure 3.3 A). Moreover, the shear-stress-induced genes PTGS2 (COX-2), KLF2, and NOS3 (eNOS) were found to be significantly upregulated under the influence of flow (Figure 3.4 F). These genes are inducible by shear stress [222], where especially KLF2 is found to be consistently expressed in cultures exposed to prolonged laminar flow and fails to be induced under the influence of a disturbed flow profile [185, 197]. As such, the results indicate that the luminal layer of the bi-layered scaffold was covered by a shear-responsive endothelial lining.

The vasoregulatory agents produced by the ECs under the influence of shear have to be transported to the vSMCs, residing in the tunica media, to be able to exert their vasoregulatory function. Not only is this communication important for the vasomotion of the vessel, it has also been previously shown that ECs promote phenotype modulation of vSMCs through signaling mechanisms [223-225]. Synthetic vSMCs can rapidly proliferate 
and synthesize ECM (collagen type I and fibronectin) [226], which is desired directly after seeding of cells to ensure complete colonization of the scaffold. In our study, this was mimicked by using MSCs for the initial seeding and pre-culture period. On the other hand, contractile vSMCs only produce small amounts of ECM (collagen IV, (tropo)elastin, laminin a5), proliferate at low rates, and can be differentiated from MSCs by the addition of biochemical factors, such as TGF $\beta[161,164,165,227]$. Specific for their function is the expression of contractile proteins aSMA, SM22a, calponin, and SMMHC [148, 174, 226, 228, 229]. In the present study, within a time-frame of 2 days after induction of differentiation, the MSCs already showed an upregulation of the proteins aSMA and calponin, and a significant upregulation of aSMA, calponin, and (tropo)elastin on a gene expression level compared to EGM controls. The early differentiation marker SM22a was not upregulated. However, this could be attributed to the high SM22a (and calponin) gene expression levels in the MSCs from one donor of the control group (p/egm, data not shown), probably caused by an early differentiation that is initiated through EC signaling or via confluencyinduced differentiation [182].

The impact of TGF $\beta$ on the differentiation of the vSMCs was further clarified by stainings of vascular-specific ECM proteins, including collagen types I, III and IV, and laminin a5. Our results revealed that the addition of differentiation medium resulted in elevated collagen type I secretion compared to the EGM controls, indicative of a more mature matrix [230]. Furthermore, we found that laminin a5, which enables cell adhesion to the surrounding basement membrane and promotes a contractile cell phenotype [66], was only located in the matrix surrounding the vSM-like cells when exposed to differentiation medium, corroborating with our result that the MSCs adopted a vSMC-like phenotype.

Together, our bioengineered graft not only holds potential to address the unmet clinical need for small-diameter blood vessels, but it could also be used as an in vitro model for cardiovascular research [231]. Whilst a large number of bioengineered grafts with different levels of complexity has been developed, in the approach taken here we uniquely combine mechanical and biochemical stimuli (i.e., flow-induced shear stress and TGF $\beta$ ) with structural components (i.e., a co-culture of vSM-like cells and ECs in biomimetic scaffolds with a fast colonization of the tunica media mimic), enabling the in vitro study of new drugs, medical devices, and disease modeling [232]. It should be noted that the current setup has some experimental restrictions. Due to the large volume of culture medium circulating in the perfusion loop, our analysis of the endothelium did not go beyond gene expression analysis and stainings. Future assessment should include functional readouts, for example through analysis of $\mathrm{NO}$ and $\mathrm{PGl}_{2}$ secretion of the ECs into the culture medium, as well as anti-thrombogenic properties of perfused constructs by exposure to whole blood or plasma. In addition, evaluations could be extended with functional read-outs of vSMC contractility in response to vasoactive agents. Finally, future directions should address the 
gradually and uni-directionally applied flow in the bioreactor system. To precondition the ECs to adapt to and withstand in vivo flow conditions, shear stress can be increased and applied in a pulsatile fashion for an even longer duration. Pulsatile flow could also benefit the maturation of the vSM-like cells via the resulting circumferential strain. This, however, would first require the mechanical optimization of the scaffold material with more elastic polymers to match its compliance with natural blood vessels.

\section{Conclusion and outlook}

In this study, we have developed a bioreactor system to culture bi-layered vascular grafts under shear stress, with a compartmentalized exposure of the graft's luminal and outer layer to cell-specific media. We demonstrated that the bioreactor allows for simultaneous layer-specific cell differentiation in our biomimetic bi-layered vascular graft, in which the natural architecture and cell phenotypes of a native vessel, in particular the tunica intima and tunica media, were successfully recapitulated by the unique scaffold design. Our results highlight that, using this co-culture system, vascular grafts from clinically relevant progenitor cell sources can be created and investigated to further improve the next generation of bioengineered vascular grafts.

\section{Acknowledgements}

This research was partially supported by ZonMw as part of the LSH 2Treat program (436001003), the Dutch Kidney Foundation, and by NWO (Netherlands Organization for Scientific Research) Graduate Program Grant (022.005.018). We also gratefully acknowledge funding for the Gravitation Program "Materials Driven Regeneration" by the Netherlands Organization for Scientific Research and thank Daimon Hall (carbonandneon. com) for support with graphical design. 


\section{Supplementary information}

Supplementary Table 1. Primers used in $q P C R$ with annealing temperature of $60^{\circ} \mathrm{C}$.

\begin{tabular}{lll}
\hline Protein/Gene & Forward sequence & Reverse sequence \\
\hline GAPDH & ATGGGGAGGTGAAGGTCG & TAAAAGCAGCCCTGGTGACC \\
aSMA/ACTA2 & ATGCCATCATGCGTCTGGAT & ACGCTCAGCAGTAGTAACGA \\
CD31/PECAM1 & GCAGTGGTATCATCGGAGTG & TCGTTGTTGGAGTTCAGAAGTG \\
Elastin/ELN & GCCAAAGCGCCCAGTTT & CGCCGACACCAGGAACTAAC \\
Ve-CAD/CDH5 & AAGCAGGCAGGTATGAGAT & TGTGTACTTGGTCTGGGTGAAG \\
vWF & TGAAGCAGACGATGGTGGATT & AGCAGGTGTCGTAAATGCAG \\
eNOS/NOS3 & CAGCACAAGAGTTATAAGATCCGC & GCACTGTCTGTGTTACTGGACT \\
SM22a/transgelin & AAGAATGATGGGCACTACCG & ACTGATGATCTGCCGAGGTC \\
Calponin/CNN & AACAACTTCATGGACGGCCT & TCTCCAGCTGGTGCCAATTT \\
KIf2 & GCACGCACACAGGTGAGA & GTCTGAGCGCGCAAACTTC \\
CoX2/PTGS2 & GCACGTCCAGGAACTCCTCA & GGGGTAGGCTTTGCTGTCTG \\
\hline
\end{tabular}


Supplementary Table 2. Markers selected for immunohistochemistry for the localization of endothelial cells, smooth muscle cells, and matrix components

\begin{tabular}{|c|c|c|c|}
\hline & Target antigen & Source & Concentration \\
\hline \multicolumn{4}{|l|}{ Primary antibodies } \\
\hline \multirow{3}{*}{$\begin{array}{c}\text { EC } \\
\text { (whole-mount) }\end{array}$} & CD31, mouse anti-human & Dako & $0.13 \mathrm{mg} / \mathrm{ml}$ \\
\hline & Ve-CAD, D867F2 & Cell Signaling Technology & 1:250 \\
\hline & vWF, 3E2D10 & Abcam & $0.5 \mathrm{ug} / \mathrm{ml}$ \\
\hline \multirow{2}{*}{$\begin{array}{c}\text { vSMC } \\
\text { (whole-mount) }\end{array}$} & CNN, MU333-UC & Biogenex & $0.15 \mathrm{mg} / \mathrm{ml}$ \\
\hline & aSMA, Clone 1A4, Cy3 & Sigma Aldrich & $0.5 \mathrm{ug} / \mathrm{ml}$ \\
\hline \multirow{2}{*}{$\begin{array}{l}\text { Cytoskeleton/adhesion } \\
\text { (whole-mount) }\end{array}$} & $\begin{array}{l}\text { F-actin, fluorescein isothiocyanata } \\
\text { labeled phalloidin }\end{array}$ & Sigma Aldrich & $0.2 \mathrm{uM}$ \\
\hline & $\begin{array}{l}\text { vinculin, monoclonal, purified clone } \\
\text { 7F9 (Part No. 90227) }\end{array}$ & Millipore & $10 \mathrm{ug} / \mathrm{ml}$ \\
\hline \multicolumn{4}{|l|}{ Matrix } \\
\hline (whole-mount) & collagen IV, goat anti human & Millipore & $40 \mathrm{ug} / \mathrm{ml}$ \\
\hline \multirow{2}{*}{$\begin{array}{c}\text { Matrix } \\
\text { (cross-section) }\end{array}$} & collagen I, mouse IgG1 & Sigma Aldrich & 1:200 \\
\hline & collagen III, rabbit lgG & Abcam & 1:250 \\
\hline \multicolumn{4}{|l|}{ Secondary antibodies } \\
\hline \multirow[t]{2}{*}{$\mathrm{EC}$} & sheep anti-mouse biotinylated & GE Healthcare & 1:300 \\
\hline & goat anti-rabbit 488 & HiLyte Fluor & $1 \mathrm{ug} / \mathrm{ml}$ \\
\hline (whole-mount) & goat anti-mouse 549 & Life Technologies & $4 \mathrm{ug} / \mathrm{ml}$ \\
\hline \multicolumn{4}{|l|}{ vSMC } \\
\hline \multirow[t]{2}{*}{ (whole-mount) } & sheep anti-mouse biotinylated & GE Healthcare & $1: 300$ \\
\hline & goat anti-mouse 546 & Life Technologies & $4 \mathrm{ug} / \mathrm{ml}$ \\
\hline \multicolumn{4}{|l|}{ Matrix } \\
\hline (whole-mount) & donkey anti-goat 647 & Life Technologies & $4 \mathrm{ug} / \mathrm{ml}$ \\
\hline Matrix & goat anti-mouse 488 & Molecular Probes & 1:500 \\
\hline (cross-section) & goat anti-rabbit 555 & Molecular Probes & 1:500 \\
\hline \multicolumn{4}{|l|}{ Tertiary antibodies } \\
\hline \multicolumn{4}{|l|}{ EC } \\
\hline (whole-mount) & Streptavidin-546 conjugate & Life Technologies & $2.5 \mathrm{ug} / \mathrm{ml}$ \\
\hline \multicolumn{4}{|l|}{ VSMC } \\
\hline (whole-mount) & Streptavidin-647 conjugate & Molecular Probes & 1:500 \\
\hline
\end{tabular}




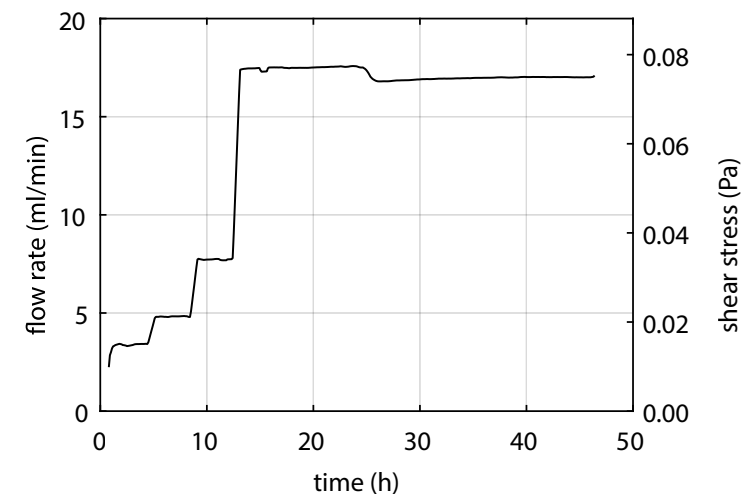

Supplementary Figure. 3.1. Flow protocol for the perfusion groups ( $p / \operatorname{tgf}$ and p/egm). The flow rate was gradually increased during the first $12 \mathrm{~h}$ to $\sim 18 \mathrm{ml} / \mathrm{min}$ and maintained for the following 36 hours. Assuming Poiseuille flow and a medium viscosity of $\mu=0.7 \mathrm{mPa} \cdot \mathrm{s}$ at $37^{\circ} \mathrm{C}$, this flow rate corresponds to a wall shear stress of $\sim 80 \mathrm{mPa}$.



Supplementary Figure 3.2. Sample processing for the selected outcome parameters. Constructs were cut in three parts for qPCR, immunocytochemistry and (immuno)histochemistry. 


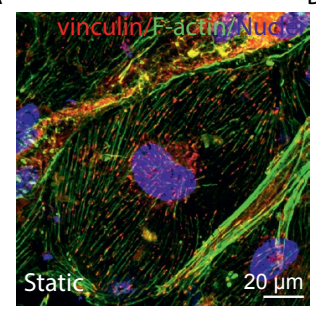



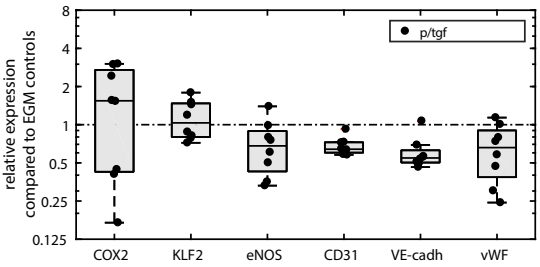

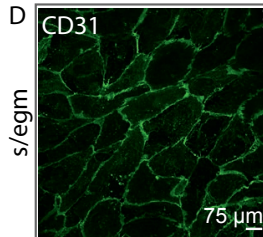
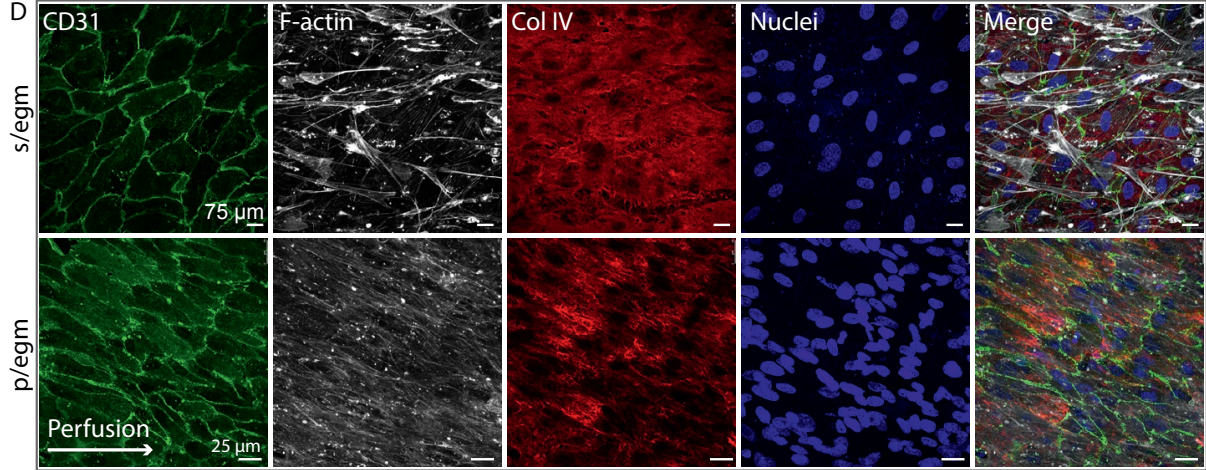

Supplementary Figure 3.3. Characteristics of the endothelium after 48 hours of s/egm and p/egm. A) Vinculin co-localized with F-actin throughout the cell cytoplasm and around the nucleus in static samples . B) VE-cadherin-positive adherence of neighboring cells with vWF appearing in dots in the absence of shear stress. C) Addition of the differentiation medium to the outer compartment did not influence the endothelium on gene expression level with no significant differences between $p /$ tgfb and p/egm. D) Representative confocal visualizations of the endothelial cells (ECFCs) cultured under static (top row) and flow (bottom row) conditions, while containing EGM medium in the outer chamber (s/egm and p/egm).


Supplementary Figure 3.4. Vascular smooth muscle cell characterization for s/tgf vs s/egm after 48 hours of static culture in the bioreactor chamber. Representative confocal visualization of the smooth muscle-like cells cultured without (top row) and with (bottom row) vSMC differentiation medium. In both situations the vSM-like cells are elongated and have a near cirumferential orientation. The $\mathrm{s} / \mathrm{tg} f$ group shows positive staining of Calponin ${ }^{+} / \mathrm{aSMA}^{+}$cells, whereas the s/egm shows negative. 


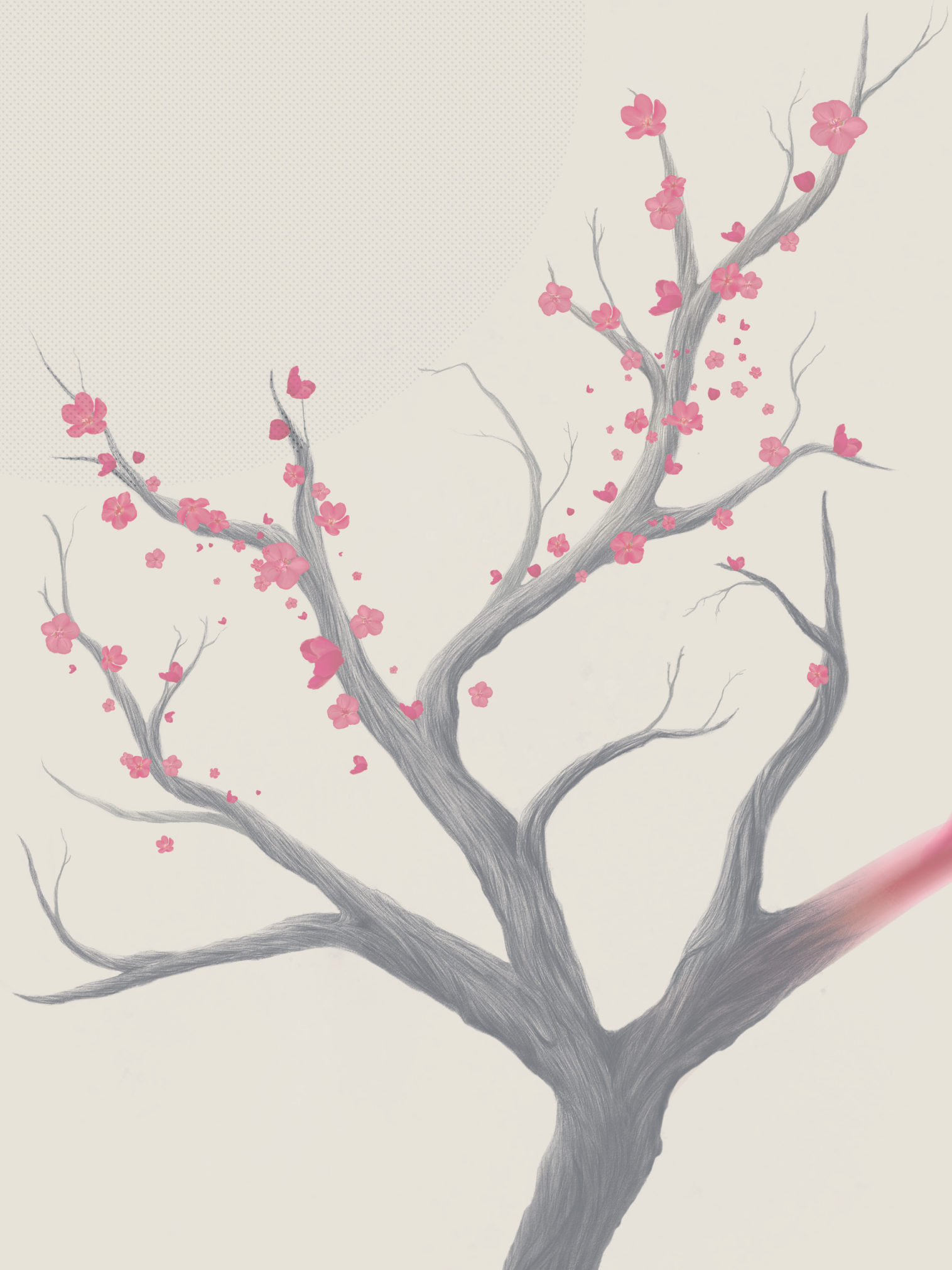




\section{Part II}

Fabrication of microscale vasculature in osteogenic

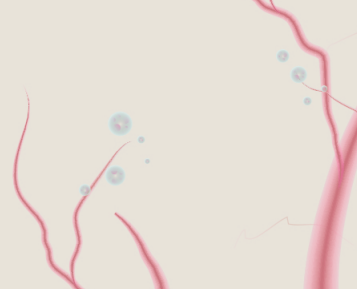





\section{Effect of donor variation on osteogenesis and vasculogenesis in hydrogel co-cultures}

this chapter is based on:

Effect of Donor Variation on Osteogenesis and Vasculogenesis in Hydrogel Co-cultures.

I. Pennings, L.A. van Dijk, J. van Huuksloot, J.O. Fledderus, K. Schepers, A.K. Braat, E.C. Hsiao, E. Barruet, B.M. Morales, M.C. Verhaar, A.J.W.P. Rosenberg, D. Gawlitta J Tissue Eng Regen Med. 2019. 13(3). 


\section{Abstract}

To introduce a functional vascular network into tissue-engineered bone equivalents, human endothelial colony forming cells (ECFCs) and multipotent mesenchymal stromal cells (MSCs) can be co-cultured. Here, we studied the impact of donor variation of human bone marrow-derived MSCs and cord bloodderived ECFCs on vasculogenesis and osteogenesis using a 3D in vitro co-culture model. Further, to make the step towards co-cultures consisting of cells derived from a single donor, we tested how induced pluripotent stem cell (iPSC)-derived human endothelial cells (iECs) performed in co-culture models.

Co-cultures with varying combinations of human donors of MSCs, ECFCs, or iECs were prepared in Matrigel. The constructs were cultured in an osteogenic differentiation medium. Following a 10-day culture period, the length of the prevascular structures and osteogenic differentiation were evaluated for up to 21 days of culture.

The particular combination of MSC and ECFC donors influenced the vasculogenic properties significantly and induced variation in osteogenic differentiation. In addition, the use of iECs in the co-cultures resulted in pre-vascular structure formation in osteogenically differentiated constructs.

Together, these results showed that close attention to the source of primary cells, such as ECFCs and MSCs, is critical to address variability in vasculogenic and osteogenic potential of the construct. The 3D co-culture model appeared to successfully generate pre-vascularized constructs and were sufficient in exceeding the $\sim 200 \mu$ m diffusion limit. In addition, iPSC-derived cell lineages may decrease variability by providing a larger and potentially more uniform source of cells for future pre-clinical and clinical applications. 


\section{Introduction}

Autologous bone transplantation is regarded as the gold standard treatment strategy to restore bone defects after trauma, infections, tumor resection or for non-unions [233]. Nevertheless, the use of autologous bone grafts is associated with infections, size mismatch, and limited availability of donor tissue. Most importantly, an adverse effect of autologous bone grafting is the introduction of new skeletal defects, accompanied by donor site morbidity and chronic donor site pain in $28 \%$ of cases [234]. Future therapies for critical size bone defects would be a great improvement if they could eliminate the need for donor tissue while ideally maintaining the efficacy of autologous bone grafting. Regenerative medicine holds the promise to develop constructs outside of the body that can be created using the desired cell types, implanted at the defect site, and designed to meet the biomechanical demands.

The maximum size of vital engineered bone constructs has traditionally been limited by the poor diffusion of oxygen and nutrients to the core regions [28, 29]. One potential strategy to solve this problem is to establish an integrated functional vascular network together with stimulation of osteogenesis and bone matrix formation, even without perfusion $[84,235]$.

One approach to create a vascular network in engineered bone tissue is to co-implant cell populations that can establish vasculature and can differentiate into osteogenic cells. Prior studies have emphasized the mutual importance of multipotent mesenchymal stromal cells (MSCs) in supporting vasculogenesis and the significance of endothelial progenitor cells (EPCs) in the stimulation of the osteogenic potential of MSCs, both in vitro and in vivo [82-86]. A specific type of EPC named "endothelial colony forming cells" (ECFCs), especially those derived from cord blood, showed robust proliferative potential and inherent vasculogenic and angiogenic capacity, and can contribute to de novo blood vessel formation in vitro and in vivo, in contrast to mature ECs [236]. However, only few studies have addressed the simultaneous formation of in vitro pre-vascular networks and osteogenesis with these type of cells [85, 235, 237].

Several key steps towards clinical translation remain to be taken. For example, the reproducibility and standardization of co-culture protocols and outcomes of selected release criteria are essential in enabling quality control of the resulting pre-vascularized tissue constructs for clinical application. At present, it is not known how the choice and combination of cells from one or different donors affects the co-culture outcomes. Data regarding ECFC heterogeneity have only been rarely reported, whereas MSCs have been widely described to have heterogeneous characteristics, potentially influenced by isolation and culture methods, donor age, donor gender and medical history [238-244]. Nevertheless, the influence of heterogeneity of donor MSCs on their specific osteogenic 
potential has only been examined in mono-layer culture models. Therefore, the first aim of the present study is to examine the effects of donor variation on the vasculogenesis and osteogenesis of a 3D co-culture model of sub-cultured primary MSCs and ECFCs.

Translating 3D co-cultures to the clinic by incorporation of autologous cells raises another critical challenge: limited cell sources. Ideally, both MSCs and ECFCs would be derived from an autologous cell source to avoid an immunogenic response upon implantation. However, the isolation of ECFCs from the adult peripheral blood is inefficient compared to isolation from cord blood, which is usually an allogeneic cell source. To date, the use of (autologous) adult peripheral blood-derived ECFCs is considered unfavorable in the clinical setting since the prevalence of ECFC is extremely low (20 times lower than in cord blood), resulting in very low isolation yields that hamper the reproducibility and viability of possible therapies $[236,245]$. Moreover, the angiogenic potential of peripheral blood-derived ECFCs appears to be substantially lower than that of their cord bloodderived counterparts $[155,246]$. Alternatively, induced pluripotent stem cells (iPSCs) could provide an unlimited source of clinically relevant, autologous endothelial cells with vasculogenic capacity. Consequently, our second goal was to evaluate the use of iPSCderived endothelial cell precursors (iECs), differentiated from several independent iPSC lines, in the Matrigel co-culture model with MSCs and assess their vasculogenic capacity and reproducibility in an osteogenic co-culture model.

In the present study, a Matrigel co-culture system in an osteogenic environment was developed in which donor dependency of vasculogenic and osteogenic cells and their behavior could be assessed.

\section{Materials and Methods}

\subsection{Isolation of MSCs}

MSCs were isolated from human bone marrow aspirates that were obtained from consenting patients $(n=7)$ (aspiration procedure was approved by the local medical research ethics committee, University Medical Center Utrecht) that underwent different surgical procedures (indicated in Table 1). These isolates are referred to as MSC1 to MSC7. Researchers were blinded to the medical history of the donors.

Aspirates were diluted 1:1 with phosphate-buffered saline (PBS) and filtered through a $100 \mu \mathrm{m}$ cell strainer. The mononuclear cell (MNC) layer was retrieved after centrifugation $(415 \times \mathrm{g}$ ) on a Ficoll-Paque (GE Healthcare) gradient (density $1.077 \mathrm{~g} / \mathrm{mL}$ ). Approximately 250,000 MNCs were plated per $\mathrm{cm}^{2}$ in MSC expansion medium consisting of a-Minimum Essential Medium (Gibco Paisley, 22561), supplemented with $10 \%$ heat-inactivated Fetal Bovine Serum (FBS) (Hyclone CSG0412 or RYG35912), $100 \mathrm{U} / \mathrm{mL}$ penicillin and $100 \mu \mathrm{g} /$ 
$\mathrm{mL}$ streptomycin (PenStrep, Gibco), $0.2 \mathrm{mM}$ L-ascorbic acid-2-phosphate (ASAP, Vitamin C) (Sigma), and $1 \mathrm{ng} / \mathrm{mL}$ basic fibroblast growth factor (rh-FGF-2; R\&D Systems). After their first passage, MSCs were either further expanded or frozen; cells from passage 4 were used in the experimental set-ups. In experiments with iECs, constructs were made with commercially available human MSCs obtained from Lonza (Poietics Human MSC) as detailed in section 2.5 .

Table 1. Overview of the multipotent mesenchymal stromal cell (MSC) donor specifics used in experimental set-ups

\begin{tabular}{lllll}
\hline Donor & Age & Gender & Type of surgery & Indicated figure \\
\hline MSC1 & 32 & Female & Spinal fusion & Figure $3-5$ \\
MSC2 & 64 & Male & $\begin{array}{l}\text { Total hip prosthesis } \\
\text { Figure } 3 \text { \& supplementary } \\
\text { Figure } 4\end{array}$ \\
MSC3 & 69 & Male & Total hip prosthesis & $\begin{array}{l}\text { Figure } 3 \text { \& supplementary } \\
\text { Figure } 4\end{array}$ \\
MSC4 & 41 & Female & Total hip prosthesis & Figure 4 \& 5 \\
MSC5 & 60 & Female & Spondolysis & Figure 4 \& 5 \\
MSC6 & 19 & Female & Total hip prosthesis & Figure 2 \\
MSC7 & 64 & Male & Total hip prosthesis & Figure 2 \\
\hline
\end{tabular}

\subsection{Isolation of ECFCs}

Cord blood of seven different donors was used (procedure was approved by the medical research ethics committee, University Medical Center Utrecht, informed consent was obtained from the mothers) as a source for the ECFC isolation (hereafter referred to as ECFC1 to ECFC7; researchers were blinded to the condition of the mother and donor child). Cord blood was diluted with PBS (1:3) and the mononuclear cell layer was retrieved after centrifugation (400 x g) on $1.077 \mathrm{~g} / \mathrm{mL}$ Ficoll-Paque gradient (GE Healthcare). $20 \mathrm{x}$ $10^{6} \mathrm{MNCs}$ were plated in a $50 \mu \mathrm{g} / \mathrm{mL}$ collagen type I-coated (BD Biosciences, rat tail) well of a 6-well plate with $1 \mathrm{~mL}$ of complete endothelial growth medium-2 (EGM-2) containing Endothelial Basal Medium-2 + SingleQuots (Lonza), $100 \mathrm{U} / \mathrm{mL}-100 \mu \mathrm{g} / \mathrm{mL}$ PenStrep and $10 \%$ heat inactivated FBS. The medium was changed daily until day 7 and then three times per week. Between weeks 2 and 4, ECFC colony outgrowth was observed. When individual colonies expanded but did not touch each other yet, the cells were trypsinized and replated to collagen type I-coated culture flasks at a density of $\sim 7,000$ cells $/ \mathrm{cm}^{2}$. Complete EGM-2 medium was used for subsequent cell expansion. After isolation, ECFCs were either expanded or frozen and used between passages 7 and 12 .

\subsection{Characterization of cell types}

\subsubsection{MSCs}

Multipotency of MSCs was examined via differentiation toward adipogenic, osteogenic, and chondrogenic lineages as described previously [237]. Briefly, osteogenesis was 
examined by staining for ALP activity (Vector SK5100 kit, Vector Laboratories) after culturing for 14 days under osteogenic differentiation medium (ODM), which consisted of a-MEM (Gibco Paisley, 22561), 10\% heat-inactivated FBS, $0.2 \mathrm{mM} \mathrm{ASAP,} 100 \mathrm{U} / \mathrm{mL}-100$ $\mu \mathrm{g} / \mathrm{mL}$ PenStrep, $10 \mathrm{mM} \beta$-glycerophosphate (Sigma), and $10 \mathrm{nM}$ dexamethasone (Sigma).

Differentiation towards the adipogenic lineage was examined by staining for lipid droplets with Oil-Red-O in iso-propanol, after 21 days of culturing in adipogenic differentiation medium (ADM). ADM consisted of a-MEM (Gibco Paisley, 22561), 10\% heat-inactivated FBS, $100 \mathrm{U} / \mathrm{mL}-100 \mu \mathrm{g} / \mathrm{mL}$ PenStrep,1 $\mu \mathrm{M}$ dexamethasone, $0.5 \mathrm{mM}$ 3-isobutyl-1methylxanthine (I7378, Sigma), $0.2 \mathrm{mM}$ indomethacin (I5879, Sigma) and $1.72 \mu \mathrm{M}$ insulin (I0516, Sigma).

Chondrogenic differentiation of the MSCs was examined by culturing them in aggregates of 250,000 cells per pellet for 3 weeks. The pellets were cultured in chondrogenic differentiation medium consisting of high glucose DMEM (Gibco Paisley, 31966), 1\% Insulin-Transferrin-Selenium (ITS) + premix (BD Biosciences), $0.1 \mu \mathrm{M}$ dexamethasone, 0.2 $\mathrm{mM}$ ASAP, $100 \mathrm{U} / \mathrm{mL}-100 \mu \mathrm{g} / \mathrm{mL}$ PenStrep and $10 \mathrm{ng} / \mathrm{mL}$ transforming growth factor $\beta 2$ (TGF- $\beta 2$ ) (R\&D Systems). Medium was changed for the first 4 days daily, afterwards every 3 or 4 days.

MSCs were phenotypically characterized by cell surface marker expression profiles with flow cytometry (BD Canto II analzyer). Expression of CD90 (THY1, FITC-conjugated; Abcam, ab124527), CD73 (AD2, PE-conjugated; Abcam, ab157335), CD105 (MEM-226, APC-conjugated; Abcam, ab60902) was confirmed as well as the absence of CD34 (4H11, APC-conjugated; Abcam, ab155377), CD45 (MEM-28, PEC-conjugated; Abcam, ab134202), CD97a (HM47, PE-conjugated; Abcam, ab177274) and CD14 (RPA-M1, FITC-conjugated, Abcam, (ab86896). IgG-matched controls were purchased from Abcam (APC, ab91358; PE, ab37392 and FITC, ab37393). Results show expression of the markers on cells based on FSC and SSC characteristics. Characterization of donor MSC 6 is shown as a representative example (Supplementary Figure 4.1).

\subsubsection{ECFCs}

Phenotypic characterization of ECFCs was performed using a BD FACSCanto II Flow Cytometer (BD Biosciences, Breda, the Netherlands). Cells were detached using accutase and checked for the following endothelial makers with: anti-hVEGFR2-PE (R\&D Minneapolis, MN), anti-hVE-Cadherin-PE (R\&D), anti-CD31-PE (R\&D), anti-CD105-PE (R\&D), anti-CD34-FITC (BD), anti-CD90 AF647 (Biolegend) and anti-CD133-PE (Miltenyi, Bergisch Gladbach, Germany), as well as absence of hematopoietic/myeloid marker expression with anti-CD45-PE (BD) and anti-CD14-PE (Biolegend, San Diego, CA). 
Additional characterization was performed by immunofluorescent staining. Cells were grown until confluency in chamber slides (Thermo Fisher, Landsmeer, the Netherlands), fixed with $4 \%$ formaldehyde and permeabilized with $0.1 \%$ Triton X-100 where appropriate. Anti-CD144 (R\&D), anti-CD31 (R\&D) and anti- von Willebrand Factor (vWF, Sigma) primary antibodies were used, secondary staining was performed with anti-Mouse AF555 and anti-rabbit AF488 secondary antibodies and nuclei were visualized with 4',6-diamidino-2phenylindole (DAPI). Images were taken with a Zeiss LSM700 Confocal Microscope. FACS profiling was performed for one ECFC donor (Supplementary Figure 4.2).

\subsection{In vitro MSC-ECFC co-cultures in Matrigel}

Co-cultures were performed in growth factor-reduced Matrigel (354230, BD Bioscience). The samples were prepared by mixing $50 \mu \mathrm{ODM}$ containing both cell types with $50 \mu \mathrm{l}$ Matrigel. Each sample of $100 \mu \mathrm{l} \mathrm{gel/ODM} \mathrm{contained} \mathrm{a} \mathrm{total} \mathrm{cell} \mathrm{volume} \mathrm{of} 625,000$ cells (ratio of 4:1 MSCs to ECFCs) and was pipetted into a 12-wells plate. The mixture was allowed to form a hydrogel at $37^{\circ} \mathrm{C}$ for at least one hour, resulting in a hemispherically shaped construct, after which $1 \mathrm{~mL}$ ODM was added on top. The medium was changed every 3-4 days. On day 10 or day 21 , hydrogels were fixed with formalin (10\%) and stored in $\mathrm{PBS}\left(4^{\circ} \mathrm{C}\right)$ until further processing.

To assess pre-vascular structure formation in large constructs, co-cultures (MSC6/MSC7ECFC7) were molded in custom-made cylindrical silicone molds (SYLGARD 184 Silicone Elastomer Kit, Dow Corning) measuring $5 \mathrm{~mm}$ diameter $\times 5 \mathrm{~mm}$ height. The co-cultures were maintained in the molds with ODM on top. The constructs were removed from the molds after 4 days of culture. These constructs were then maintained in ODM until fixation on day 10.

Because of limitations in the numbers of competent sub-cultured primary cells, the influence of donor variation on vasculogenic and osteogenic potential had to be studied with different sets of MSC-ECFC combinations. The combinations used to evaluate the influence on vasculogenesis and early osteogenesis (ALP) are displayed in Table 2 ( 3 donors for each cell type, $n=3$ gels per combination). Table 3 indicates the combinations used to study the influence of MSC and ECFC variation on the osteogenic properties of the constructs (i.e., osteonectin expression and mineralization) ( $N=3$ independent repetitions, $\mathrm{n}=2$ gels per combination). 
Table 2. MSCs (M) and ECFCs (E), each derived from 3 different donors (Table 1) were co-cultured in various combinations for assessment of vasculogenic differentiation. Note. MSC: multipotent mesenchymal stromal cell; ECFC: endothelial colony forming cell.

\begin{tabular}{lll}
\hline ECFC 1 & ECFC 2 & ECFC 3 \\
\hline M1E1 & M1E2 & M1E3 \\
M2E1 & M2E2 & M2E3 \\
M3E1 & M3E2 & M3E3 \\
\hline
\end{tabular}

Table 3. Overview of MSC (M) and ECFC (E) donorcombinations for assessment of osteogenic differentiation. Note. MSC: multipotent mesenchymal stromal cell; ECFC: endothelial colony forming cell.

\begin{tabular}{lll}
\hline ECFC 4 & ECFC 5 & ECFC 6 \\
\hline M1E4 & M1E5 & M1E6 \\
M4E4 & M4E5 & M4E6 \\
M5E4 & & \\
\hline
\end{tabular}

\subsection{Generation of iECs from iPSCs and iEC/MSC co-cultures}

Endothelial cells were differentiated from human iPSCs and are referred to as iECs, as previously described [247, 248]. Briefly, two iPSC lines (control episomal iPS cell lines eWT1323-2 and eWT-BJ2 [249]) were used and both differentiated twice into iECs; hereafter referred to as iEC1-1; iEC1-2 and iEC2-1; iEC2-2 respectively. FACS of KDR (VEGFR2) and PECAM (CD31) double positive cells (PECAM1-AF488; clone M89D3,\#558068 and KDRAPC; clone 89106, \#560871) was performed on the single cells derived from the embryoid bodies, as described in [250]. The sorted PECAM ${ }^{+} / \mathrm{KDR}^{+}$cells were subsequently cultured on fibronectin-coated plates with complete ECM (Sciencell). iECs were combined in coculture with commercially available MSCs obtained from Lonza (Poietics Human MSC; 24655, PT-2501), and used between passages 6 and 10 in the co-cultures. Co-cultures were created and cultured as described in section 3.4.

\section{6. (Immuno)histochemical staining}

\subsubsection{CD31, NG2, and a-SMA staining in hydrogels}

To demonstrate the presence of endothelial cells in the co-cultures, CD31 immunofluorescence staining was conducted on half a sample fixed on day 10. After permeabilization, non-specific protein binding was blocked before incubating (1 hour) with the primary anti-CD31 antibody $(0.13 \mathrm{mg} / \mathrm{mL}$ mouse anti-human CD31, M0823, Dako) with or without anti-NG2 chondroitin sulfate proteoglycan antibody $(0.001 \mathrm{mg} / \mathrm{mL}$, Merck Millipore, AB5320). After washing with $0.1 \%$ Tween in PBS, one hour of incubation with the secondary antibody (1:200, sheep antimouse biotinylated, RPN1001v1, GE Healthcare) was performed. The samples were incubated with streptavidin Alexa Fluor 488, $2 \mathrm{mg} / \mathrm{mL}$ (Invitrogen), together with the anti-a-smooth muscle actin (a-SMA) fluorescently labeled antibody (Clone 1A4, Cy3 $0.5 \mu \mathrm{g} / \mathrm{mL}$, C6198, Sigma Aldrich). The $5 \mathrm{~mm} \varnothing \times 5 \mathrm{~mm}$ constructs were incubated overnight $\left(4^{\circ} \mathrm{C}\right)$ with the antibodies. 
Finally, nuclei were counterstained with 4',6-diamidino-2-phenylindole dihydrochloride (DAPI) staining $100 \mathrm{ng} / \mathrm{mL}$ (Sigma) for 15 minutes at room temperature.

\subsubsection{CD31 staining on paraffin sections}

Sections $(5 \mu \mathrm{m}$ ) were cut from the samples ( $5 \mathrm{~mm}$ diameter $\times 5 \mathrm{~mm}$ constructs, the groups from Table 3 and iEC co-cultures) after embedding in paraffin. Following de-waxing and rehydration steps, antigen retrieval was performed on the sections by boiling in citrate buffer (10 mM, pH 6) for 15 minutes, washing in demi water and $0.1 \%$ Tween in PBS. Peroxidase blocking was carried out in $0.3 \% \mathrm{H}_{2} \mathrm{O}_{2}$ in PBS (10 minutes). After blocking (15 minutes, 5\% BSA/PBS), slides were incubated overnight at $4{ }^{\circ} \mathrm{C}$ with either the antiCD31 primary antibody $(0.13 \mathrm{mg} / \mathrm{ml}$ mouse anti-human, Dako, M0823) or concentration matched isotype control (isotype mouse igG1, Dako, X0931). The samples were incubated with the secondary antibody $(6.5 \mathrm{mg} / \mathrm{ml}$ rabbit anti-mouse HRP, Dako, P0260) for 1 hour. Then they were exposed to the tertiary polymeric HRP-linker antibody (brightVision poly-HRP-anti Rabbit, Immunologic DPVO55HRP) for 1 hour, followed by the addition of 3,3'-diaminobenzidine (DAB) substrate solution, which is transformed to brown staining facilitated by HRP. Optionally, the staining can be combined with an a-SMA staining (1:300 Monoclonal Anti-Actin, a-Smooth Muscle - Alkaline Phosphatase antibody, Sigma, clone 1A4), color development with ALP-kit (Vector SK5100 kit, Vector Laboratories) before boiling in citrate buffer. Nuclei were counterstained with Mayer's hematoxylin (Merck). Finally, the sections were dehydrated and mounted with Depex or aquamount.

\subsubsection{Osteocalcin/osteonectin staining on paraffin sections}

Upscaled cylindrical constructs and iEC containing cultures were tested for osteogenic differentiation by staining for the osteoblast marker osteocalcin (OCN). Effects of variation of the MSC donor on osteogenic differentiation were examined via the osteogenic marker osteonectin (ON) in the set of co-cultures in Table 3. ON production was determined after 10 and 21 days of culture. Peroxidase blocking was performed before the sections were boiled in citrate buffer $(10 \mathrm{mM}, \mathrm{pH} 6)$ for 20 minutes. After protein blocking (15 minutes, $5 \% \mathrm{BSA} / \mathrm{PBS}$ ), slides were incubated with the primary anti-ON antibody ( 9 $\mathrm{ug} / \mathrm{mL}, \mathrm{DSHB}$, AON-1 was deposited to the DSHB by Termine, J.D. (DSHB Hybridoma Product AON-1)) and incubated at RT for 2.5 hours. Sections were washed 3 times for 5 minutes with PBSTween, after which they were incubated with an anti-mouse HRP-labelled polymer for 1 hour (Dako, Envision, K400011). Addition of DAB substrate solution resulted in a brown staining, facilitated by HRP. Nuclei were counterstained with Mayer's hematoxylin (Merck). Finally, the sections were dehydrated and mounted with Depex.

The osteocalcin staining was performed according to a similar protocol with the following changes: antigen retrieval was performed on the sections by incubation in pronase (Sigma, 10165921001) (30 minutes, 1 mg/ml) and hyaluronidase (Sigma, H2126; 30 min, 
$10 \mathrm{mg} / \mathrm{mL})$. The incubation with primary antibody $(0.02 \mathrm{mg} / \mathrm{mL})$ mouse anti-bovine osteocalcin (clone OCG4, Takara M044) was performed overnight at $4^{\circ} \mathrm{C}$. The sections were then incubated with the secondary antibody GAM-HRP $(5.5 \mathrm{mg} / \mathrm{L}$ goat anti-mouse, Dako, P0447) for 1 hour.

\subsubsection{Von Kossa staining on paraffin sections}

Von Kossa staining was used to visualize mineral deposition in the co-cultures (Table 3) after 21 days of in vitro culture. Non-demineralized and rehydrated sections were incubated with $1 \%$ silver nitrate in demi water (Fisher Scientific) directly under a regular light bulb for 1 hour, prior to washing with $5 \%$ sodium thiosulfate in demi water (Alta Aesar). Mayer's hematoxylin nuclear counterstaining was performed before dehydration and mounting in Depex.

\subsection{Alkaline phosphatase activity in hydrogels}

ALP was used to indicate early osteogenesis, as it's activity is associated with committed osteoprogenitor cells. For detection of ALP activity (in the samples shown in Table 2) on day 10, the fluorescent Vector SK5100 kit was used. In order to link the ALP activity with the vasculogenesis in one half of a construct, the remaining half of the construct was used for the CD31 staining (Table 2). Constructs were permeabilized and incubated with the kit's Red Substrate in the dark followed by washing in demi water.

\subsection{Quantification of the pre-vascular structures}

The influence of donor variation on angiogenic properties was further assessed by quantifying the total length of all CD31-positive structures in the images (length in pixels). Images of the CD31-stained co-cultures ( $n=3$ per group) of Table 2 were captured (Olympus BX60, Cell-F software) and processed in Adobe Photoshop CS6. The levels were individually adapted by eliminating over- and under-exposed pixels following conversion of the images to black and white. Subsequently, the images were inverted (brightness -150 and contrast 100). The resulting files were batch processed in the freeware program "Angioquant" [251]. All images had individually-optimized processing settings for smoothening, segmentation with automatic thresholding, and pruning of structures below 25 pixels. Then, the total length of the pre-vascular structures (total length in one image of a construct at day 10) was assessed.

\subsection{Statistical analyses}

Statistical analyses were performed with GraphPad Prism 6.01. Angioquant outcomes were tested for the significant influence of donor variation on the total structure length with a two-way ANOVA with multiple comparisons of the mean, when varying the donor of the MSCs or the ECFCs. A Tukey HSD Post-hoc test was used to correct for multiple 
comparisons. Differences between the experimental groups were considered statistically significant if $p<0.05$. Outcomes are presented as means $\pm S D$.

\section{Results}

\subsection{Identification and characterization of MSCs and ECFCs}

As expected, the bone marrow-derived MSCs showed a fibroblast-like morphology, their multi-lineage potential was confirmed, and their CD-marker expression profile was consistent with accepted criteria for MSCs [252] (Supplementary Figure 4.1 A-D). ECFCs organized into their characteristic colonies with rounded cell morphology and exhibited a cobblestone morphology upon passaging. The ECFCs showed high expression of endothelial/hematopoietic stem cells markers (Supplementary Figure 4.2).

\subsection{Formation of 3D pre-vascular structures in MSC-ECFC osteogenic co-cultures}

Hemispherical $100 \mu \mathrm{l}$-sized co-cultures of MSCs with ECFCs in Matrigel, cultured in osteogenic differentiation medium, produced CD31-positive 3D pre-vascular networks by day 10 (Figure 4.1 A). At day 21, an even more extensive network with an increased number of junctions was observed (Figure 4.1 B). Pseudopodial processes, on sprouting tip cells, were observed in the constructs at both day 10 and 21, indicating ongoing angiogenesis (Figure $4.1 \mathrm{C}$ ). In addition, pericytic mural cells adjoining the CD31-positive pre-vascular structures were visualized by NG2 (Figure 4.1 D) and a-SMA detection (Figure 4.1 E).

The nuclear staining of the co-cultures demonstrated that not all cells contributed to vasculogenesis or differentiated towards mural cells (Figure 4.1 F). Most likely, these cell nuclei belong to cells which are committed to the osteogenic lineage (as presented in Supplementary Figure 4.4). Mono-cultures of either MSCs or ECFCs did not show prevascular network formation or a-SMA-positive network formation (Supplementary Figure 4.3). 

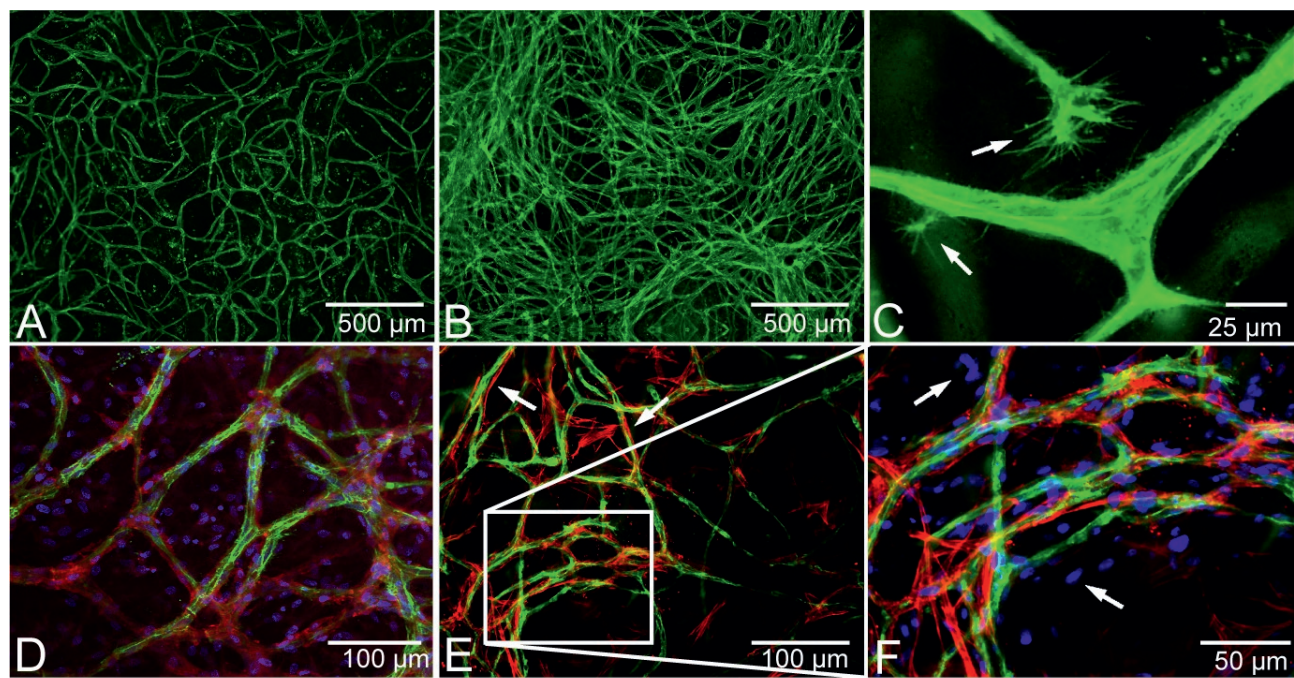

Figure 4.1. Pre-vascular structures and mural-like cells in osteogenic MSC-ECFC co-cultures in Matrigel. CD31-positive staining in green shows endothelial structures after A) 10 and B) 21 days in Matrigel cultures in osteogenic differentiation medium. C) Sprouting tip cells were observed with filopodia (arrows), D) NG2 positive cells (red) align with the prevascular structures (CD31, green), and E) aSMA-positive cells (red, arrows), supported the endothelial structures (green, CD31). F) Highermagnification image from E) showing cell nuclei (blue, arrows) of cells that did not stain for CD31 (green) or a-SMA (red). Images are representative of at least three independent experiments with various MSC and ECFC donor combinations.

\subsection{Co-cultures in larger 3D constructs}

To confirm 3D vascular network formation in constructs of a considerable size, $5 \mathrm{~mm} \varnothing \times 5$ mm cylindrical-shaped MSC-ECFC osteogenic Matrigel co-cultures were established. Prevascular structures were recognized throughout these well-shaped cylindrical constructs (Figure 4.2 A-E). Cross-sectional images from the center of the construct showed similar structures as observed in the $100 \mu \mathrm{l}$-sized, thin samples of Figure 4.1, including the a-SMAlined endothelial structures (Figure 4.2 C). In addition, osteogenic differentiation of cells in these large constructs was demonstrated by positive OCN staining throughout the construct (Figure 4.2 F). 

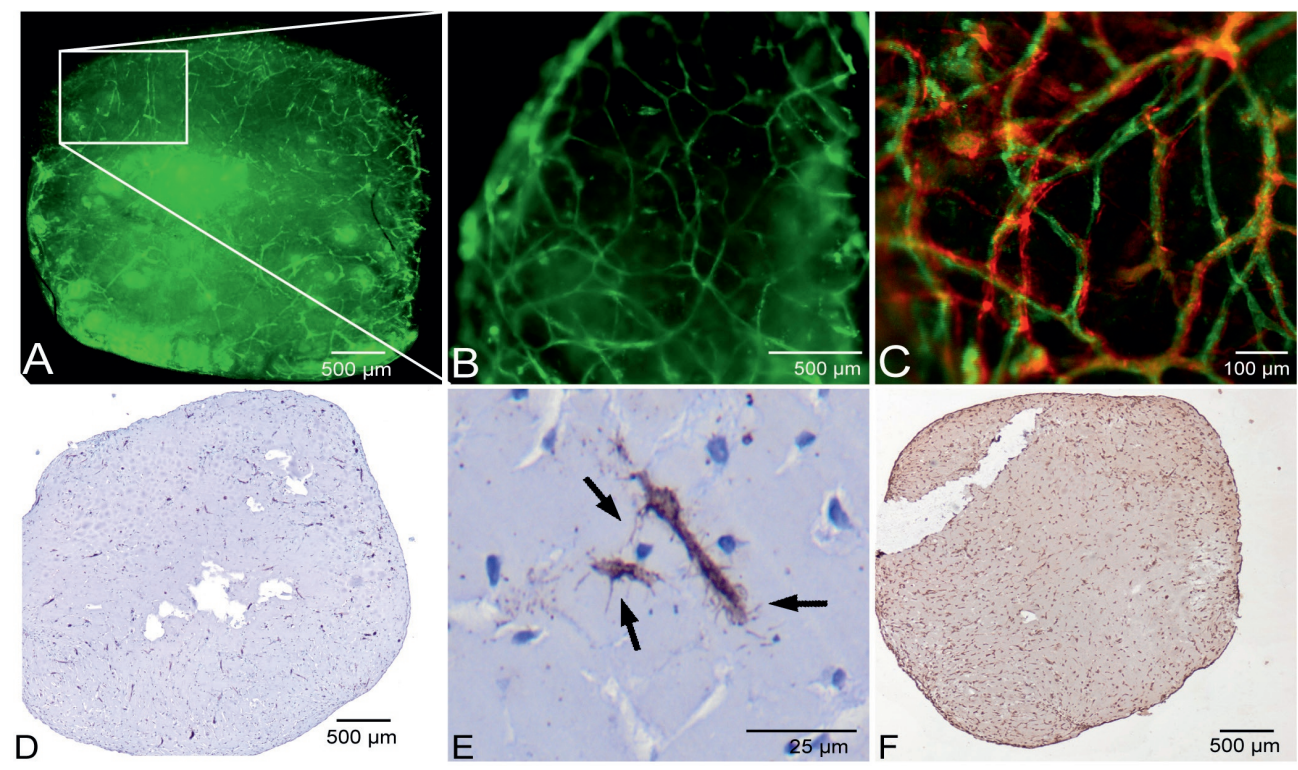

Figure 4.2. Vasculogenesis and osteogenesis in larger MSC-ECFC 3D matrigel constructs at day 10. A-B) CD31-positive structures were found throughout the volume of the $5 \mathrm{~mm} \varnothing \times 5 \mathrm{~mm}$ Matrigel whole-mount constructs, C) adjoined by a-SMA-positive cells. D-E) On sections, CD31 staining shows prevascular network formation and sprouting cells, and F) osteocalcin deposition (brown) was observed throughout the complete cross-section of the construct. Images are representative of two independent experiments with two MSC donors with one ECFC donor (M6E7 and M7E7).

\subsection{The effects of donor variation on pre-vascular network formation}

All evaluated donor combinations (Table 2) of MSCs with ECFCs, cultured in $100 \mu$ l-sized hemispherical Matrigel constructs, produced a CD31-positive 3D pre-vascular network by day 10 (Figure $4.3 \mathrm{~A}$ ). Nonetheless, the total length of the vascular structures (in pixels) was significantly different in the various combinations, indicating that the angiogenic properties differed when varying the donor combinations (Figure 4.3 B). More specifically, when varying the ECFC donor in the co-culture combinations, the total vessel length was affected within one MSC donor group for some donor combinations (Figure 4.3 B) (M1E1 vs M1E3; M2E1 vs M2E2; M2E2 vs M2E3; M3E1 vs M3E3).

Similarly, by varying MSC donor while the ECFC donor was unchanged, a significant difference in the pre-vascular network length was observed (e.g., M1E1 vs M2E1 and M2E1 vs M3E1). From this, it can be concluded that both the MSCs' and ECFCs' donor individually influence the length of the pre-vascular structures, thus the angiogenic properties of the co-culture. Overall, the specific donor combination of MSC-ECFC rather than one cell type has the overhand in determining the length of the pre-vascular network; i.e., the cell types react differently when combined with cells from different donors. 


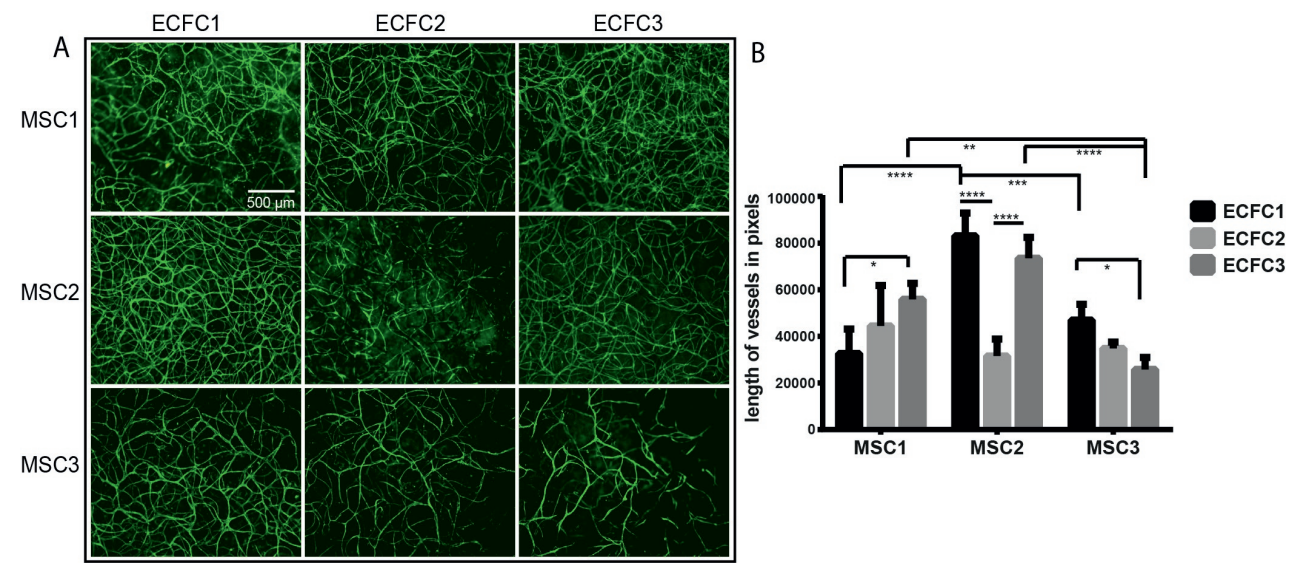

Figure 4.3. Influence of MSC and ECFC donor variation on the formation of pre-vascular structures. A) The pre-vascular networks formed by nine donor combinations (Table 2) are shown at day 10 (CD31 staining in green). The scale bar represents $500 \mu \mathrm{m}$ for all images in A. Images are each representative of triplicates. B) Quantification of the pre-vascular structures revealed significant differences in total structure length (pixel number) among the donor combinations. ${ }^{*} p<0.05, p^{* *}<0.01,{ }^{* * *} p<0.001$, $* * * * p<0.0001$.

\subsection{Osteogenic differentiation in MSC-ECFC co-cultures}

To confirm simultaneous osteogenic differentiation and vasculogenesis in the co-cultures, the constructs analyzed in Figure 4.3, were also evaluated for ALP activity. Qualitative analysis confirmed that the nine co-culture combinations displayed ALP activity throughout the construct (Supplementary Figure 4.4). Among these combinations, minimal variation in the intensity of the ALP staining was visible. An increase of ALP activity was found in MSC-ECFC co-cultures compared to the corresponding MSC monocultures (Supplementary Figure 4.4, insets).

To further confirm osteogenic differentiation in the co-cultures, ON expression was evaluated in the MSC-ECFC combinations described in Table 3. Figure 4.4 shows the ON expression in the co-cultures after 21 days, as well as for MSC mono-culture controls (Figure $4.4 \mathrm{~A}$, insets). In all combinations, ON expression was also detected after 10 days (Supplementary Figure 4.5), with an increase in cellular and matrix staining intensity after 21 days of culture. Addition of ECFCs to the MSC-containing constructs did not have an explicit influence on this ON deposition at day 21 . In constrast, at day 10 , the cocultures showed more ON expression than the corresponding MSC mono-cultures. Also, co-cultures stained for ON at 10 days showed formation of cell clusters, positive for ON, which may be indicative of early bone nodule formation and corresponding osteoblast maturation [253]. 
No clear influence of variation of the MSCs donor or ECFCs donor was found on the ON expression, at both time-points 10 or 21 days. A relatively small difference in ON expression could be observed among MSC mono-cultures. Simultaneous osteogenic differentiation and vasculogenesis in one construct was confirmed by expression of ON and CD31/aSMA on consecutive slides of donor combination M5E4 (Figure 4.4 B-C).
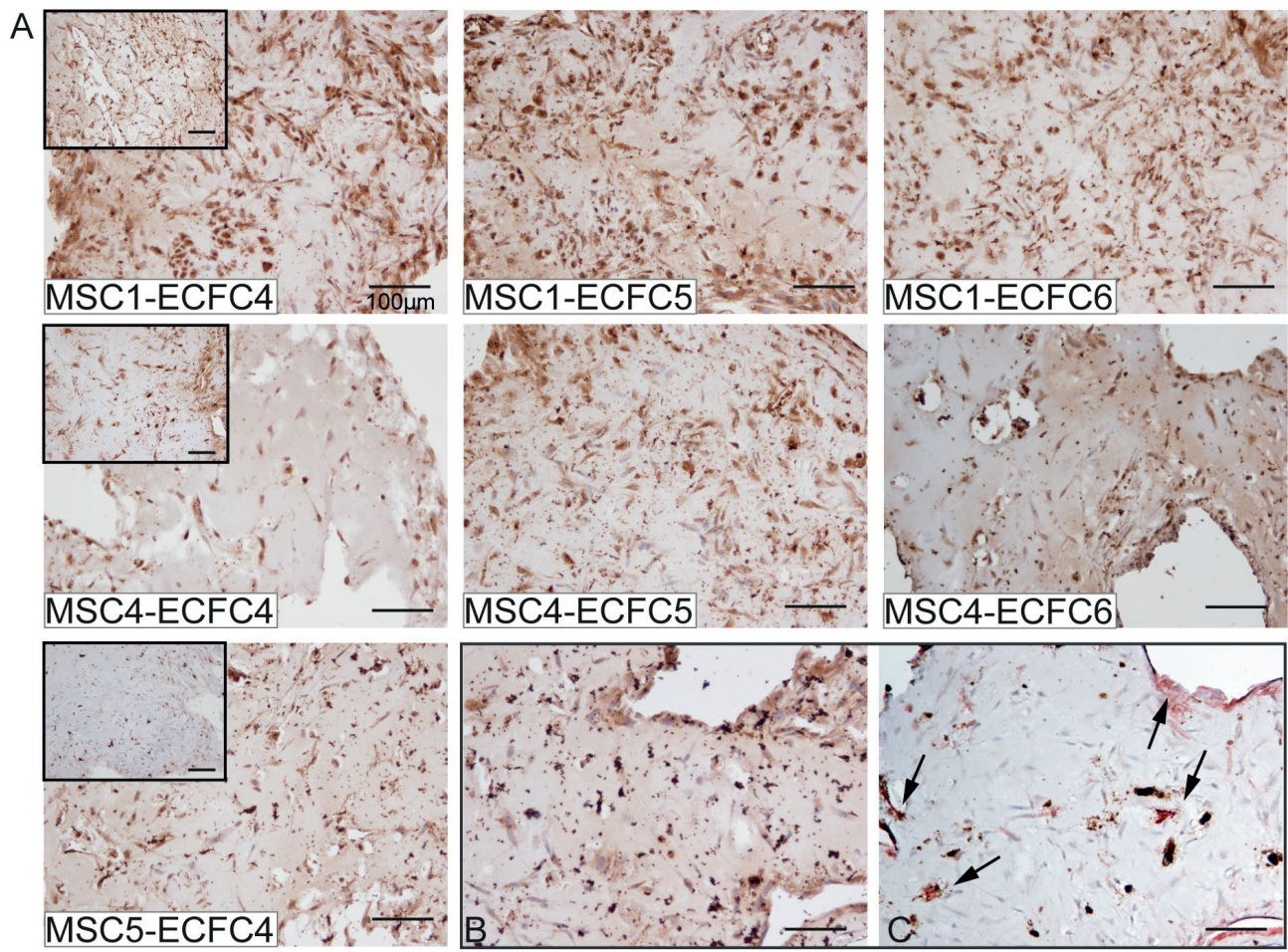

Figure 4.4. Osteonectin deposition in MSC-ECFC co-cultures in Matrigel at day 21. A) Co-cultures from three different MSC donors and from three ECFC donors revealed that variation of the ECFC donor did not strongly affect ON deposition at day 21, as well as the variation of the MSC donor. Insets contain the corresponding ON-stained MSC mono-culture controls, where a difference in ON staining intensity can be appreciated. Images are representative of three independent experiments ( $n=2$ /experiment). B) ON protein expression is shown in brown, with the corresponding C) CD31 ${ }^{+}$ vascular structures (brown) and aSMA positive mural cells (red) as detected in consecutive slides of donor combination M5E4. Black arrows indicate positive staining. Scale bar represents $100 \mu \mathrm{m}$.

To determine if the osteogenic differentiation also resulted in construct mineralization, von Kossa staining was performed on day 21 on the donor combinations of Table 3 (Figure 4.5). All co-cultures showed staining indicating mineralization, but only in selected combinations calcified nodule formation could be observed already. MSC1 only showed mineralized nodule formation when combined with ECFC donor 5, but was solely developed in a late stage during culture (observed via bright-field microscopy, 
data not shown). In contrast, MSC4 contained calcified nodules when combined with all ECFC donors. Nodule formation for MSC4-containing combinations was already observed at day 10 of culture, however formation of new nodules did not continue over the period of culture for M4E5. MSC5 containing co-cultures also showed early nodule formation that increased over time, resulting in a highly mineralized matrix and nodule formation (Figure $4.5 \mathrm{~A}$ ). The combinations with mineralized nodules and matrix (M4E4 - M4E6 - M5E4) also showed a homogeneous staining of the matrix in the ON staining (Figure $4.4 \mathrm{~A}$ ). Interestingly, in corresponding MSC mono-cultures, the MSCs did not display mineralization by day 21 (Figure 4.5, insets). Overall, the extent of mineralization was determined mostly by the choice of MSC donor. In contrast, the variation of the ECFC donor did only influence the level of mineralization in the MSC1 co-culture constructs; in all cases, ECFC addition did induce (a faster) mineralization. Presence of CD31/aSMAdouble positive structures in a mineralized matrix was confirmed by positive staining on consecutive slides of donor combination M5E4 (Figure 4.5 B-C), on the same location as presented in Figure 4.4 B-C. 

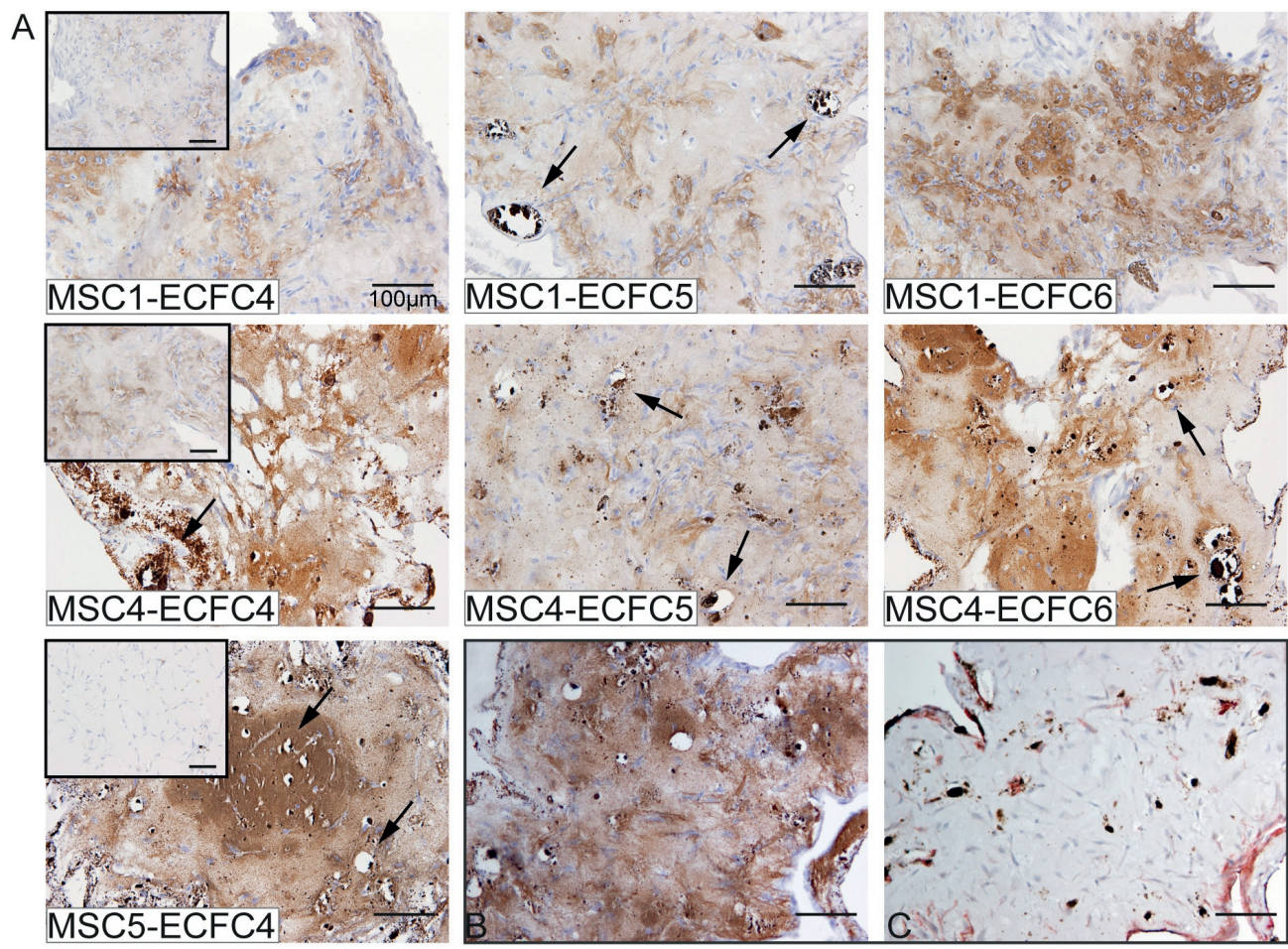

Figure 4.5. Mineralization in MSC mono-cultures and MSC-ECFC co-cultures in Matrigel at day 21. A) The images show increased von Kossa staining (brown) in the co-cultures, compared to corresponding MSC monocultures (insets) in all cultures. Variation of the ECFC donor did not influence the extent of mineralization. Variation in the MSC donor did influence the extent of mineralization (M1E4, M4E4, M5E4 and M1E6, M4E6). Mineralization in brown is shown, with the corresponding C) CD31+ vascular structures (brown) and aSMA positive mural cells (red) as detected in consecutive slides of donor combination M5E4. Black arrows indicate positive staining. Images are representative of three independent experiments ( $n=2 /$ experiment). Scale bar in images represents $100 \mu \mathrm{m}$.

\subsection{Implementation of iECs/MSCs in Matrigel co-cultures}

Finally, we evaluated if human iPSC-derived endothelial cell precursors (iECs) could be used in 3D co-cultures, if they would show de novo vasculogenesis, and if different iECs showed variable reproducibility in angiogenesis and/or osteogenic support in cocultures. CD31 staining after 10 days of co-culture revealed that the 4 combinations of iECs with MSCs (2 separate derivations of iECs from 2 iPSC lines) showed comparable levels of vasculogenesis when reviewed qualitatively (Figure 4.6 A). Also, a-SMA-positive cells (Supplementary Figure 4.6 A) and CD31-positive cells (Supplementary Figure 4.6 B) could be detected throughout the constructs via immunohistochemistry. OCN staining of the same constructs demonstrated that the four combinations of iEC-MSC were all capable of undergoing osteogenesis (Figure 4.6 B). Additionally, nodule mineralization of the constructs was confirmed (Figure $4.6 \mathrm{C}$ ). The co-cultures of the different combinations mineralized where the group with iEC1-2 showed the most intense staining. Overall, 
all co-cultures containing iECs could successfully undergo vasculogenesis and support osteogenesis, suggesting that different iEC lines can show reproducible outcomes.

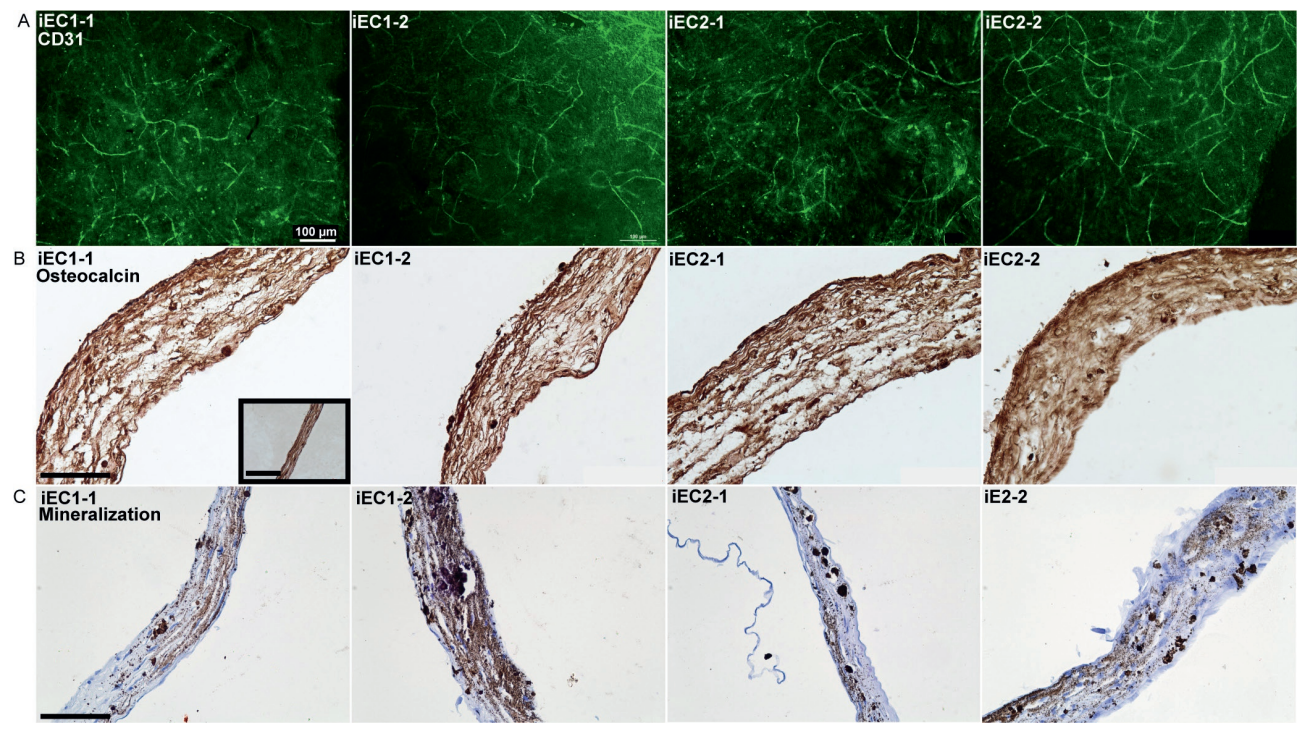

Figure 4.6. Effects of induced pluripotent stem cell-derived endothelial (iEC) variation in iEC-MSC cocultures on vasculogenesis and osteogenic differentiation. Co-cultures of MSCs with iECs support A) CD31-positive endothelial network formation (green); B) deposition of osteocalcin (brown) (inset is MSC mono-culture); and C) mineralization (brown) in all combinations, with highest mineral content in iEC1-2. All co-cultures used Poietics Human MSCs. Images are representative of 4 independent experiments. Scale bars in images represent $100 \mu \mathrm{m}$; scale bar in insets represents $50 \mu \mathrm{m}$.

\section{Discussion}

Current in vitro tissue engineering strategies are largely confined to the creation of small constructs. Generation of an inherent functional 3D vascular network within the tissue engineered construct is considered essential for the creation of larger constructs. Here, we used a matrigel/hydrogel-based model to create vascular networks in an osteogenic construct, and used these constructs to investigate an important aspect of robustness of experimental outcomes.

The model consistently generated 3D pre-vascular networks supported by mural cells in osteogenically differentiated constructs. This phenomenon was also observed when larger constructs were generated, in which the size reached the often described diffusion limit of $200 \mu \mathrm{m}[28,29]$. The addition of ECFCs to the MSCs enhanced the osteogenic differentiation and mineralization. In contrast to most co-culture models (reviewed by Liu et al., 2015), no endothelial cell stimulants were added to the culture medium, which suggests that induction of vasculogenesis can occur via cross-communication of the embedded MSCs. We found that under the influence of osteogenic factors a subpopulation of the MSCs 
was committed to the osteogenic lineage while other subpopulations of the MSCs and/or ECFCs supported endothelial network formation by differentiation into mural cells.

This finding is in line with other reports of successful vasculogenesis in co-cultures in osteogenic medium [85, 235, 237]. Our results indicate an extensive context-dependent communication between MSCs and endothelial cells, creating a mutually stimulating environment likely involving (growth) factors and/or cell-cell contacts, influencing both mural cell differentiation, and vasculo-angiogenic and osteogenic processes [154, 254256].

A close interplay between these growth factors and the opposing receptors signifies the balance in communication between the discussed cell types. When deriving the cell types from different donors, this delicate balance in communication is likely to be affected by donor variability. Accordingly, variations in outcomes of the vascular length and level of osteogenic differentiation were observed in our co-cultures (Figure 4.3, 4.4 and 4.5). Here, we showed that the specific MSC-ECFC combination, rather than one cell type, had the overhand in determining the levels of vasculogenesis. Furthermore, the MSC donor was a decisive factor in the amount and rate of osteogenic differentiation/mineralization in the co-culture model. As such, an (im)balance in the cross-communication might be linked to the influence of donor variation on vasculogenesis and osteogenesis.

Another explanation for the variation in osteogenic differentiation levels is that MSCs of different donors can react differently to dexamethasone that is present in the used osteogenic medium, resulting in diverging ALP expression, different expression profiles of osteogenic markers and mineralization [241, 257, 258]. This also matches previous observations of Siddappa etal., 2007 and Phinney et al., 1999, where a number of parameters was identified, such as the passage number and time point of medium change, that could additionally influence the maturation of the tissue engineered (osteogenic) constructs. This variability in steroid responsiveness may also affect the MSCs' communication with the ECFCs. Thus, variable reactions of MSCs to osteogenic medium can alter the osteogenic differentiation rate, but could also influence their signaling to the ECFCs.

Important aspects of translation towards clinically relevant constructs that consist of multiple cell types, as presented here, are the balanced cross-communication, reproducibility of their performance, but also the limitation of cell sources and amount of cells that can be isolated can cause setbacks. Cord blood-derived ECFCs have been shown to be superior to the ECFCs isolated from peripheral blood in their proliferative and neoangiogenic capacity, and are present in sufficient amounts in cord blood. Despite these promises, there are limitations in its use as an autologous cell source for adults since it requires biobanking of the cell-product directly after birth. On the other hand, since the 
isolation of adult ECFCs is hampered by their low prevalence in peripheral blood, they could hardly serve as a robust autologous cell source for therapies for all patients in a clinical setting [236]. As an alternative, patient-specific iPSC (or HLA superdonor)-derived ECs can serve as an unlimited source of clinically relevant cells [259]. The co-culture model in this study allowed the creation of constructs containing iECs, which were shown to be a feasible alternative for ECFCs. Our data provide proof of concept of reproducible implementation of $\mathrm{IECs}$ in osteogenic differentiation models and showed its potential of de novo blood vessel formation in an in vitro co-culture setting. Hints of robustness of this approach are demonstrated by the similarity in differentiation of four different iECs with MSCs of one donor. In the future, the effect of donor variation on differentiation in coculture models could potentially be diminished by incorporation of ECs and MSCs derived from a single iPSC line of the patient.

Thus, by deriving iECs from patient-derived iPSCs, the drawbacks of using cord bloodderived ECFCs can potentially be overcome. Moreover, iPSCs exhibit an unlimited proliferation potential and therefore present an attractive (autologous) cell source for future regenerative treatments [78]. Furthermore, iECs might be able to reduce variation, as iPSCderived endothelial cells were shown to be produced with high batch uniformity [260] and less variance than primary ECs [248], which has also been demonstrated in our iECMSC co-cultures (Figure 4.6).

So far, several research groups have reported successful creation of pre-vascular structures derived from iPSCs. iECs have been shown to repopulate decellularized tissue engineered vascular structures [261], (self)assemble into perfusable tubular structures [260, 262] and form 3D networks in vitro [263, 264] and in vivo [78]. Orlova et al., 2014 succeeded in co-culturing of iPSC-derived ECs and PSC-derived pericytes in a 2D environment, and demonstrated that iPSC-derived ECs were able to functionally integrate into embryonic zebrafish vasculature. Nevertheless, before iPSCs can be integrated in therapies in clinical settings, safety concerns must be addressed.

Overall, our study demonstrates that care should be taken when varying donors of cells in co-culture models, as donor variation can affect cell differentiation and thus the reproducibility of results. It is imperative that several important hurdles towards clinical translation of functional pre-vascularized bone constructs are taken. This does not only include the choice of (autologous/allogeneic) cell sources but also upscaling of size, reproducibility and standardization of (co-)culture protocols and release criteria. Our in vitro 3D co-culture model is an accessible method to explore new regenerative strategies to overcome these hurdles and move towards in vivo applications. 


\section{Acknowledgements}

This research was partially supported by an NWO (Netherlands Organization for Scientific Research) Graduate Programme Grant (022.005.018). D.G. received funding from the Dutch Technology Foundation, STW (Veni 11208). E.C.H received support from a Doris Duke Charitable Fund Clinical Scientist Development Award (2014099).

\section{Supplementary Figures}


Supplementary Figure 4.1. Characterization of the Multipotent Mesenchymal Stromal Cells from bone marrow (MSC6). The primary human MSCs were found to be positive for the stem cell markers CD90, CD73, and CD105, and negative for CD14, CD34, CD45 and CD79a. The MSC was shown to be able to differentiate into all 3 lineages (osteogenic - ALP; Adipogenic - oil Red O; chondrogenic - Safranin O). 



DAPI

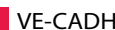

DAPI

CD31

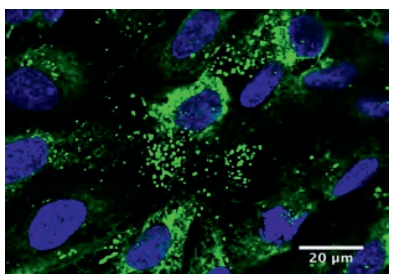

DAPI

vWF

Supplementary Figure 4.2. Characterization of the Endothelial Colony Forming Cells after isolation from cord blood. With flow cytometry, the ECFCs were found to be positive for endothelial/ hematopoietic stem cell markers CD31, CD34, KDR, CD90, CD133, VE-Cadherin, and CD105 and negative for CD14 and CD45 (blue, red histograms display isotype controls). Also, immunofluorescent staining confirmed the presence of VE-cadherin, CD31 and vWF protein (bottom row, respectively), the top row shows isotype staining controls. 


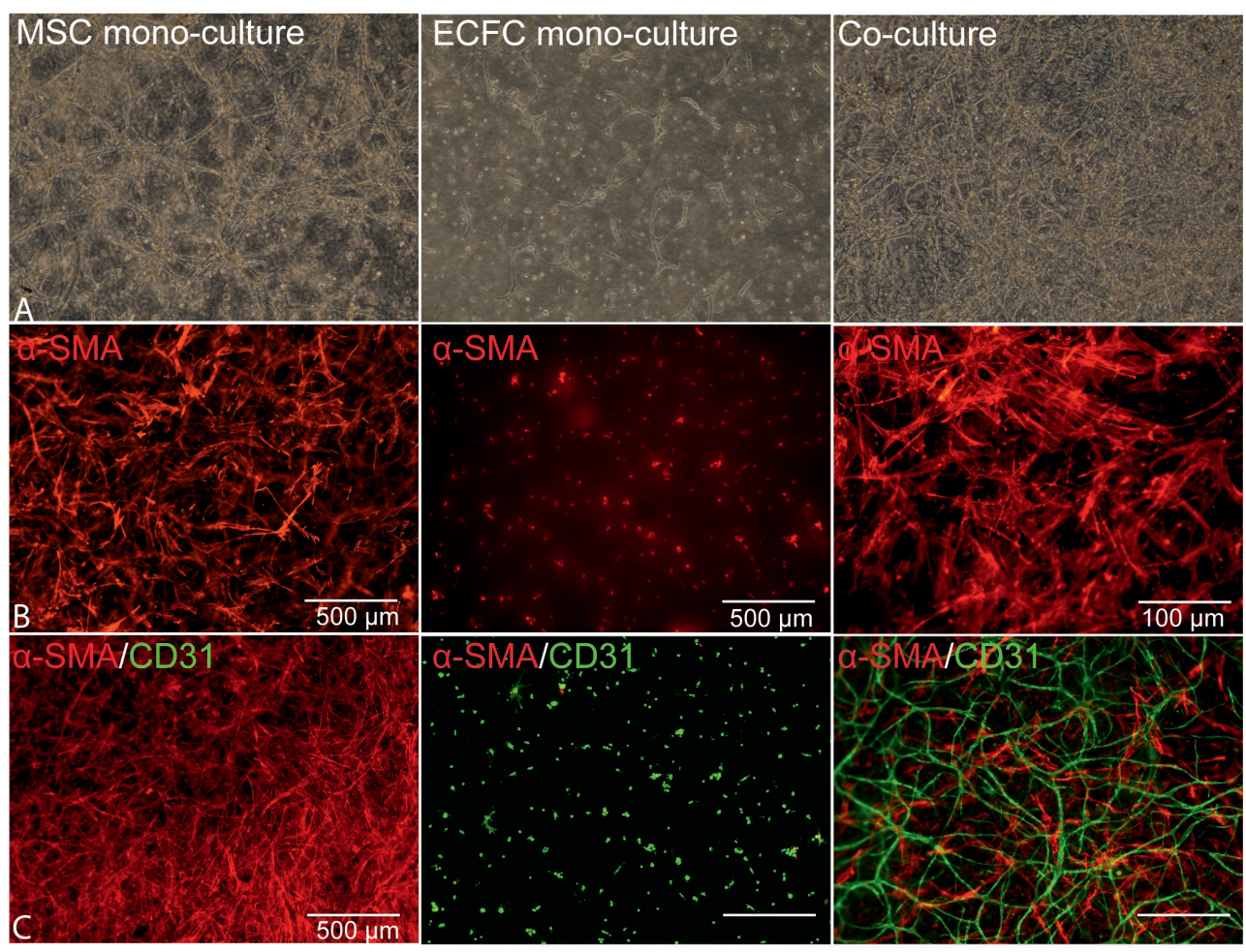

Supplementary Figure 4.3. $a$-Smooth muscle actin and CD31 positive cells in mono- and co-cultures in Matrigel. Mono-culture controls did not exhibit endothelial network organization. A) At day 3 , structures were observed by light microscopy in co-cultured constructs, but also in mono-cultures of MSCs in Matrigel. B-C) Stainings for CD31 (green) and a-SMA (red) revealed that co-cultures contained endothelial networks with adjoined a-SMA-positive cells while the structures in MSC mono-cultures were only a-SMA positive at day 10. In addition, the Matrigel cultures of ECFCs alone did not show any structure formation. 


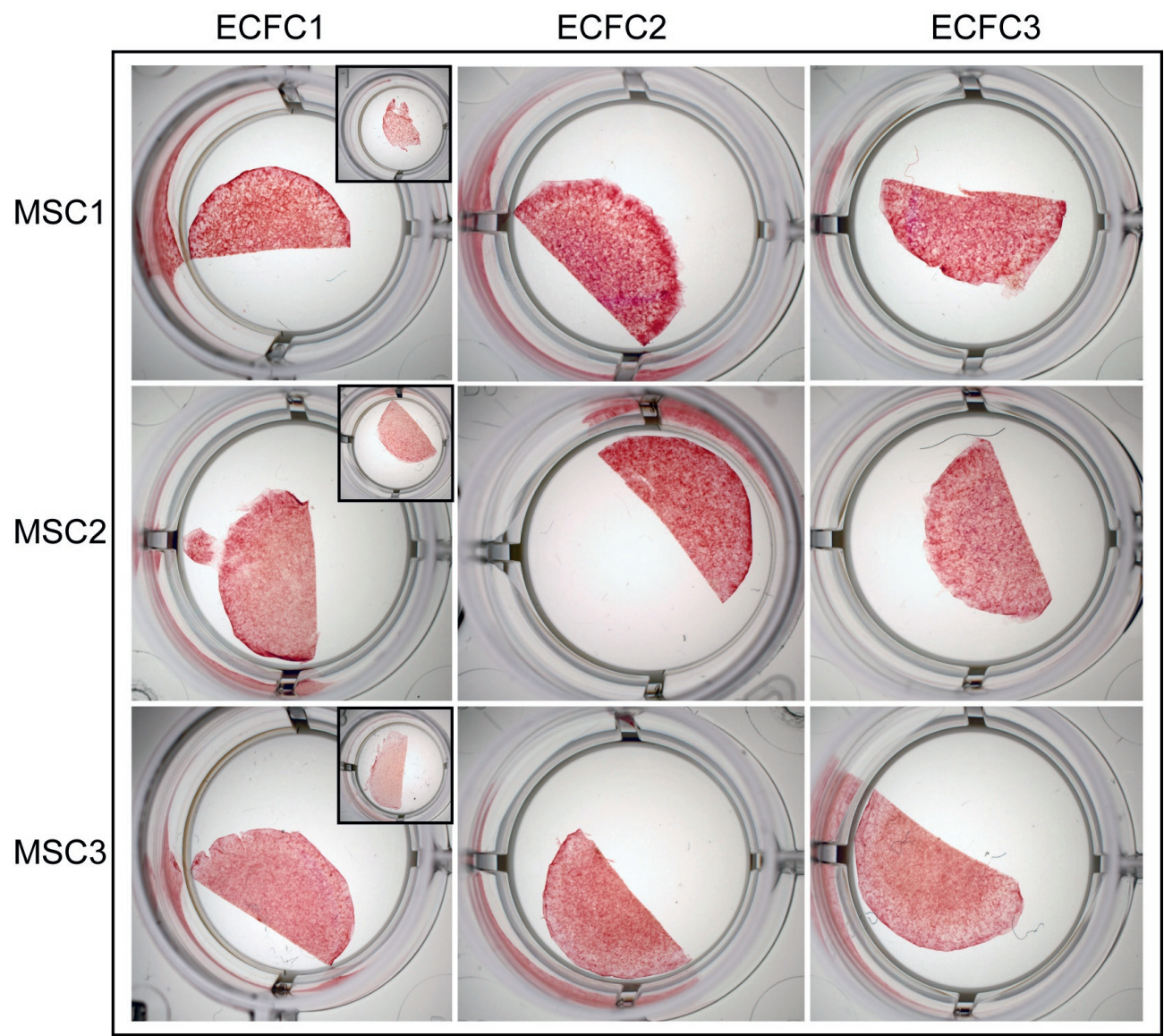

Supplementary Figure 4.4. Alkaline Phosphatase (ALP) staining in nine MSC-ECFC donorcombinations (Table 2) after 10 days of culture. All donor combinations showed ALP activity (red) to a similar extent. Images are representative of the triplicates and ALP activity of corresponding MSC mono-culture controls can be found in the insets. 

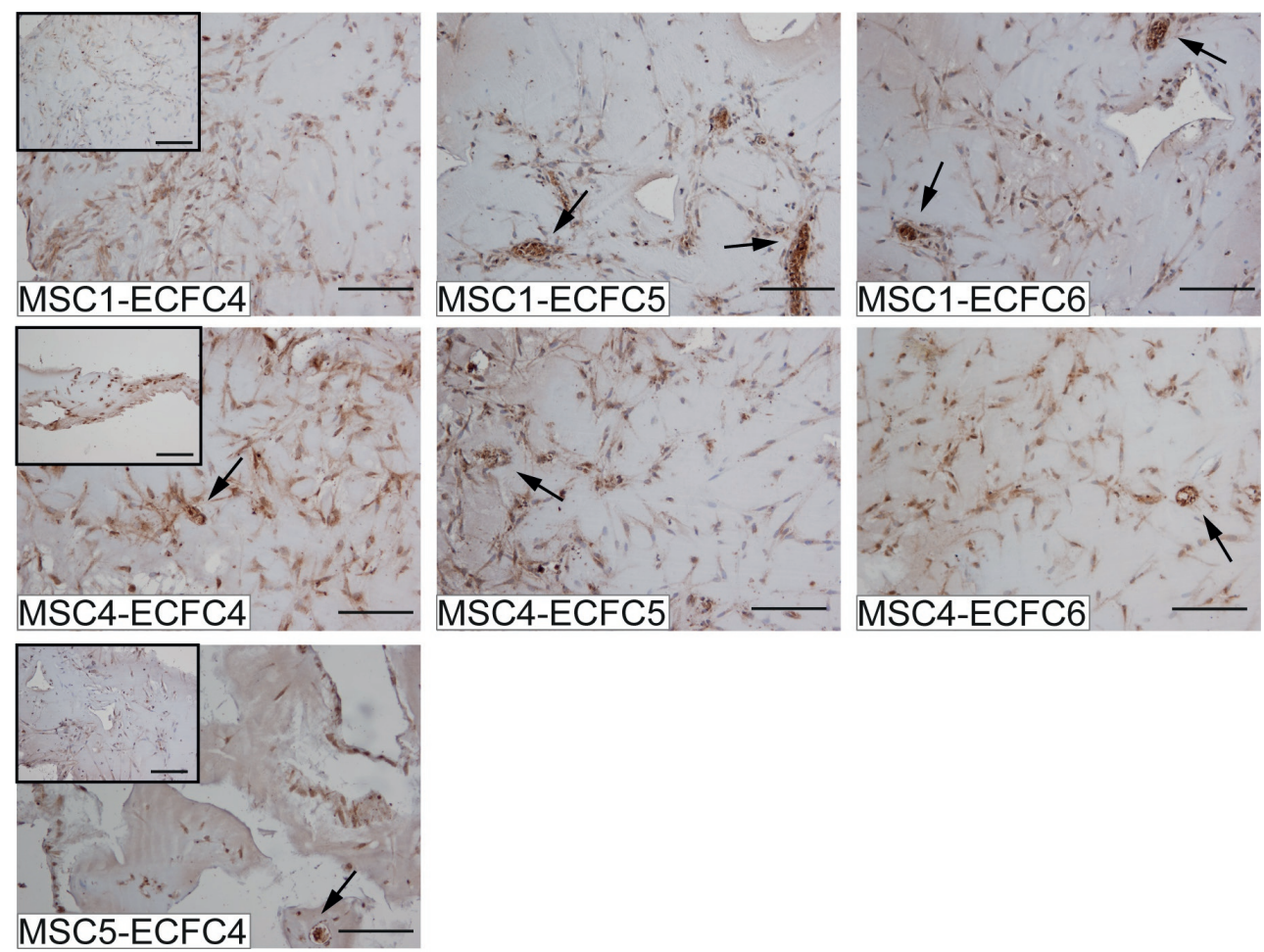

Supplementary Figure 4.5. Osteonectin protein expression in co-cultures at day 10. MSCs show ON expression when cultured alone in 3D Matrigel cultures in ODM. Mono-cultures show a slight difference between donors (insets). Addition of ECFCs enhanced the expression of ON slightly and resulted in clusters of cells (black arrows).

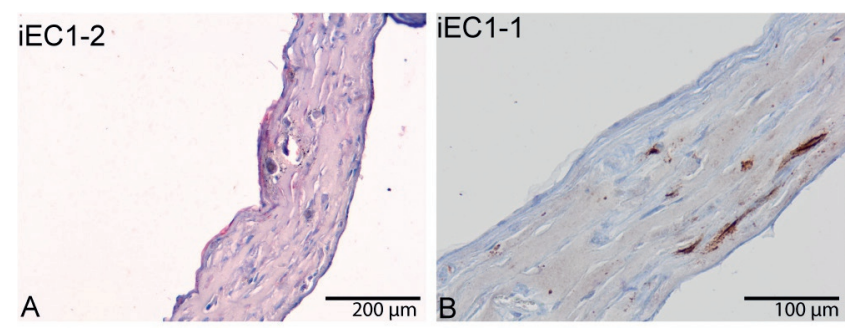

Supplementary Figure 4.6. a-Smooth muscle actin and CD31 positive cells in iEC-MSC Matrigel co-cultures. A) Representative paraffin sections of the iEC1-2 co-culture showed a-SMA positive structures (in red). B) iEC1-1 co-cultures show CD31 positive staining in paraffin sections. 


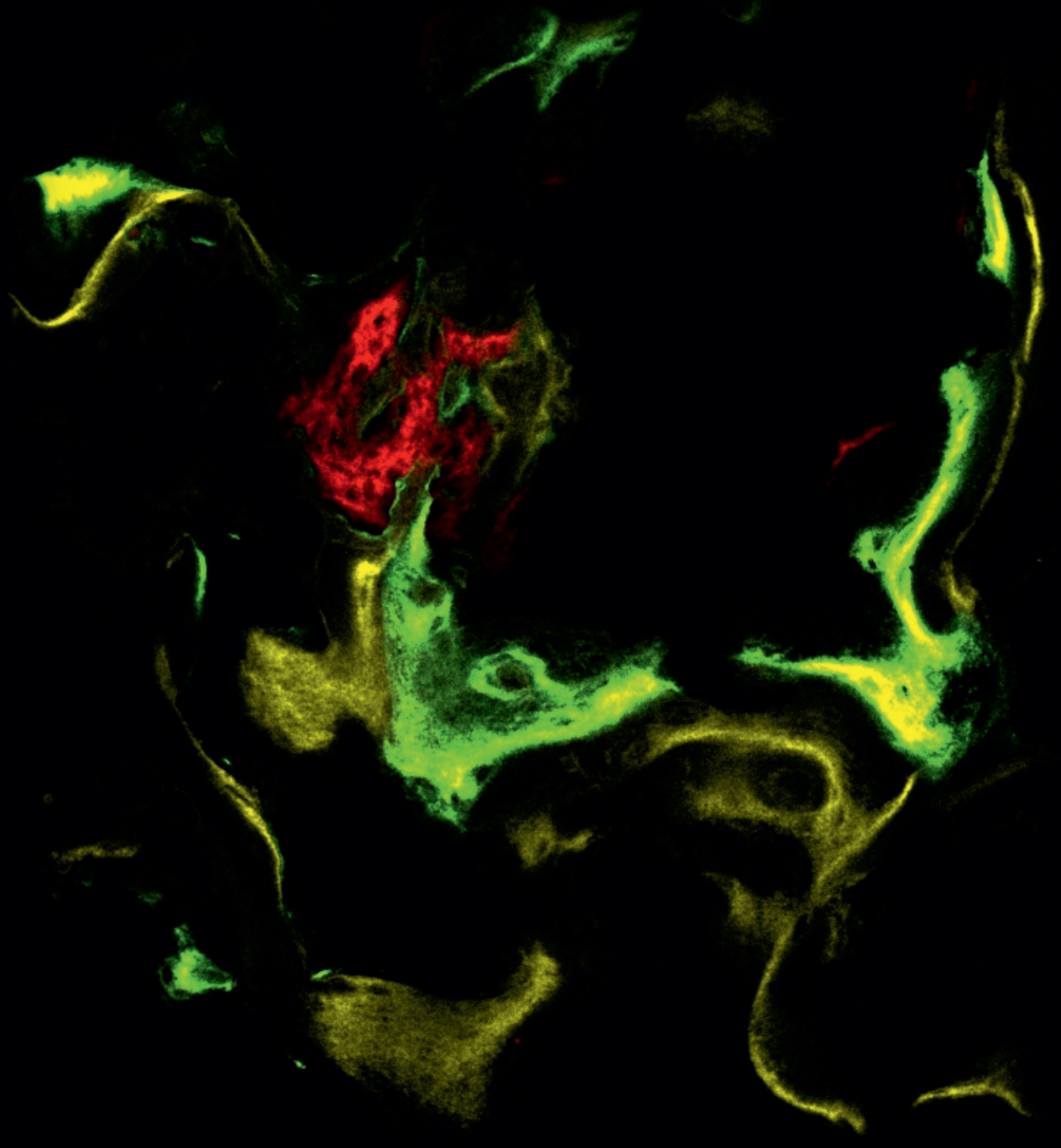




\section{In vitro pre-differentiation of pre- vascularized bone constructs containing calcium phosphates accelerates the onset of in vivo bone}

formation



In vitro pre-differentiation of pre-vascularized bone constructs containing calcium phosphates accelerates the onset of in vivo bone formation.

I. Pennings, A. Longoni, Q. Vallmajo-Martin, C. Cheng, M. Ehrbar, A.J.W.P Rosenberg, D. Gawlitta

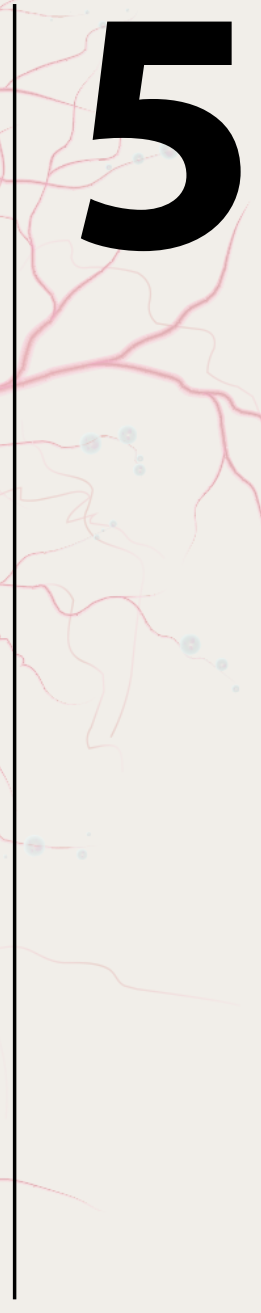




\section{Abstract}

Including a functional vascular network in living osteogenic tissue constructs with calcium phosphates is a prerequisite to upscale their size to clinically relevant dimensions. As yet, it is unknown whether, and for how long, an in vitro predifferentiation period is required for such complex co-cultures. Therefore we aimed to elucidate if it would benefit in vivo vascularization and bone formation.

Pre-vascularization of biphasic calcium phosphate constructs was achieved by coculturing multipotent mesenchymal stromal cells with endothelial colony forming cells in a gelatin-PEG hydrogel. Constructs were osteogenically pre-differentiated for 1,6 or 14 days and analyzed for their osteogenic and vasculogenic characteristics. Hereafter, constructs were implanted subcutaneously in immunocompromised rats for 8 weeks and evaluated for the extent of bone formation, matrix maturation and vascular state.

Prolonging the pre-differentiation period affected the onset of bone formation and the source of bone forming cells. The onset was accelerated with the extended predifferentiation period, and the maturity of the formed bone matrix post-implantation was enhanced. Pre-differentiation further favored the retention of the donor cells within the construct. Retained donor cells were actively forming the new bone tissue. This indicates that by controlling the pre-differentiation state of a construct, the participation of the implanted cells in bone formation can be tuned. 


\section{Introduction}

Reconstruction of critical-size (mandibular) bone defects is a complex procedure as its success largely depends on the restoration of the lost form and function. Reconstructions are often carried out by transplantation of autologous bone grafts to the defect site. However, this procedure has several drawbacks such as restricted graft availability and donor-site morbidity. The need for a secondary skeletal defect and associated complications at the donor site, as well as size mismatch at the transplantation site, can be prevented by developing engineered bone constructs. This can be achieved by the use of synthetic bone graft substitutes or bioengineered constructs.

Commonly, calcium phosphate (CaP) minerals are the main constituents of synthetic bone substitutes as they constitute $60-70 \%$ of the native bone tissue, with the collagens and non-collagenous proteins as the remaining components [265,266]. Within the bone, $\mathrm{CaPs}$ are responsible for the mechanical stability and resistance to compression, while collagen provides elasticity and resistance to tension [265]. Among the synthetic bone graft substitutes, bioceramics resemble the native bone minerals the best, and often comprise osteoinductivity and/or osteoconductive characteristics, which is favorable for in vivo bone formation $[266,267]$. The pool of synthetic bone ceramics includes a wide variety of materials, ranging from, but not limited to, bioactive glass, $\mathrm{CaP}$ cements, hydroxyapatite (HA), $\alpha$ - or $\beta$-tri-calcium phosphate ( $\alpha$ - or $\beta$-TCP) ceramics and bi-phasic calcium phosphates $(B C P)$ thereof $[24,268]$.

In the last decade, it has been indicated that the enrichment of CaP ceramics with osteogenic (precursor) cells results in higher rates of bone regeneration, and thereby also improves the ceramic-based bone regeneration in small animal models [269, 270]. This emphasizes that a vital parameter required for high rates of (ectopic) bone formation is the osteogenic differentiation of cells seeded in calcium phosphate constructs [271-274]. To preserve this added effect of the cells, it is important to keep the cells alive when scaling up the construct size. To explain, restricted diffusion of gas and exchange of nutrients across $\mathrm{cm}$-scale tissues currently limits the size of living engineered tissue substitutes. In the natural situation, this problem is solved by the presence of an extensive capillary bed, which brings the cells in mature bone within the vicinity of the nutrient supply $(\sim 100-150 \mu \mathrm{m})$ [30]. Incorporating such vascular networks in bioengineered constructs would benefit the in vitro and in vivo survival. Various strategies have been designed with this specific aim, varying from the utilization of smart scaffold design, the delivery of angiogenic factors to stimulate in vivo vascularization, or via vitro pre-vascularization of bone constructs $[27,71,275]$.

So far, for CaP based constructs in specific, only few approaches have been designed to induce or enhance vascularization. The most described strategies are based on in 
vivo vascularization methods approaches and concern the addition of proangiogenic growth factors and bioinorganics to the CaPs, or to use surgical approaches (i.e. freeflaps, extrinsic pre-implantation models or arteriovenous bundles) [276] (reviewed in [7, 277-279]). In vivo vascularization occurs after implantation of the construct in a highly vascularized recipient site, and usually depends on extrinsic vascularization. Although the extrinsic blood vessels eventually sprout into the engineered construct, their growth rate is low. Consequently, insufficient blood is supplied to the core tissue of the construct, which then turns necrotic [280]. Moreover, migration and functionality of osteogenic cells largely depend on the existence of a vascular system and associated osteo-vascular crosscommunication. Of pertinence here, poor vascularization can impede osteogenesis [37, $38,278]$. This leads to believe that cell-based CaP constructs require an in vitro intrinsic capillary network before implantation, to maintain cellular viability and to contribute to a faster connection to the surrounding network.

The effects of CaPs on in vitro angiogenesis [84, 281-284] or in vivo vascularization [272, 278, $283,285-293$ ] have already been established. Mostly positive effects were reported on in vivo bone formation and construct vascularization. Nonetheless, these effects were shown when comparing osteoprogenitor monocultures in CaP constructs to those enriched with endothelial cells. Moreover, the reported outcomes are difficult to interpret and compare as they use various cell pre-differentiation periods. This variable is of specific interest when moving towards pre-vascularized CaP-based constructs since the development of a functional microvasculature takes time. Moreover, when aiming to engineer large tissue constructs, engineering of a functional, perfused vascular network is essential to ensure survival of the embedded cells, both in vitro and in vivo. Therefore, we aim to improve our understanding of the influence of osteogenic pre-differentiation of co-cultured CaPcontaining constructs on vascularization and bone formation after implantation. The results will help to define which level of osteogenic-vasculogenic differentiation, prior to implantation, promotes in vivo vascular integration and bone formation.

To realize this premise, biphasic CaP granules were integrated in an osteogenic prevascularized gelatin-PEG co-culture model. This model consists of an innovative gelatin-PEG (gelPEG) hydrogel, accommodating a co-culture of human endothelial colony forming cells (ECFCs) and multipotent mesenchymal stromal cells (MSCs) in an osteogenically stimulating environment [294]. The co-culture is reliant on the aforementioned tight coupling of angiogenesis and osteogenesis, where the extensive cross-communication between the osteogenically-induced MSCs and the ECFCs naturally triggers vasculogenesis, without the supplementation of endothelial growth factors [206]. With this model, the following question will be answered: if, and for how long this construct should be pre-cultured to optimize in vivo tissue regeneration. Therefore, the constructs were i) osteogenically pre-cultured for either 14 days with the aim to promote 
extensive osteogenic differentiation with an established pre-vascular network, ii) 6 days to endorse early commitment to the osteogenic lineage with an established vascular network, or iii) directly implanted on day 1, without an osteogenic differentiation or established pre-vascular network. Constructs without cells were included as controls. The three pre-culture strategies were compared by implanting the constructs subcutaneously in immunocompromised rats, followed by evaluation of the bone formation rate, matrix production and the state of the vasculature at 8 weeks post-implantation.

\section{Materials and Methods}

\subsection{Study design}

To study the effect of the length of the pre-differentiation period on in vitro tissue formation, constructs were made with a combination of human MSCs and ECFCs in a gelatin-PEG hydrogel system [294] with CaP granules (35/65\% HA/ $\beta$-TCP) (Figure $5.1 \mathrm{~A}$ ). They were osteogenically pre-differentiated for 21 days and were evaluated for their in vitro osteogenic differentiation based on alkaline phosphates (ALP) activity, osteonectin (ON) expression and matrix mineralization. The in vitro screening of the pre-culture period was carried out in hydrogel droplets $(75 \mu \mathrm{L})$, and the outcomes were used to determine the required pre-differentiation time for the up-scaled in vivo cylindrical constructs (10 $\mathrm{mm} \varnothing, 4 \mathrm{~mm}$ height) prior to implantation (Figure 5.1 A,D).

To check whether the various in vitro pre-differentiation periods would enhance new bone formation in vivo, constructs pre-cultured for 1 (T-1), 6 (T-6) or 14 (T-14) days were implanted subcutaneously in nude rats (Figure $5.1 \mathrm{~B}$ ). As a control, CaP/hydrogel constructs without cells were taken along. The T- 1 group represented constructs that were not differentiated, T- 6 was comprised of constructs that had an early commitment to the osteogenic lineage and T-14 contained constructs that were osteogenically differentiated but did not show matrix mineralization yet. Constructs cultured for 21 days showed to be mineralized and were therefore not included in the in vivo evaluation; results of this time point are only shown in the supplementary data. Rats were followed up for 8 weeks, and groups were compared for their ability to form bone and support vascularization. Micro-computed tomography $(\mu C T)$ (tissue mineralization, shown in supplementary data), fluorochrome analysis (time-line of mineralization), histology (bone, vasculature and extracellular matrix) and Microfil (vasculature) were used for evaluation of explanted constructs (Figure 5.1 C, E-F). 

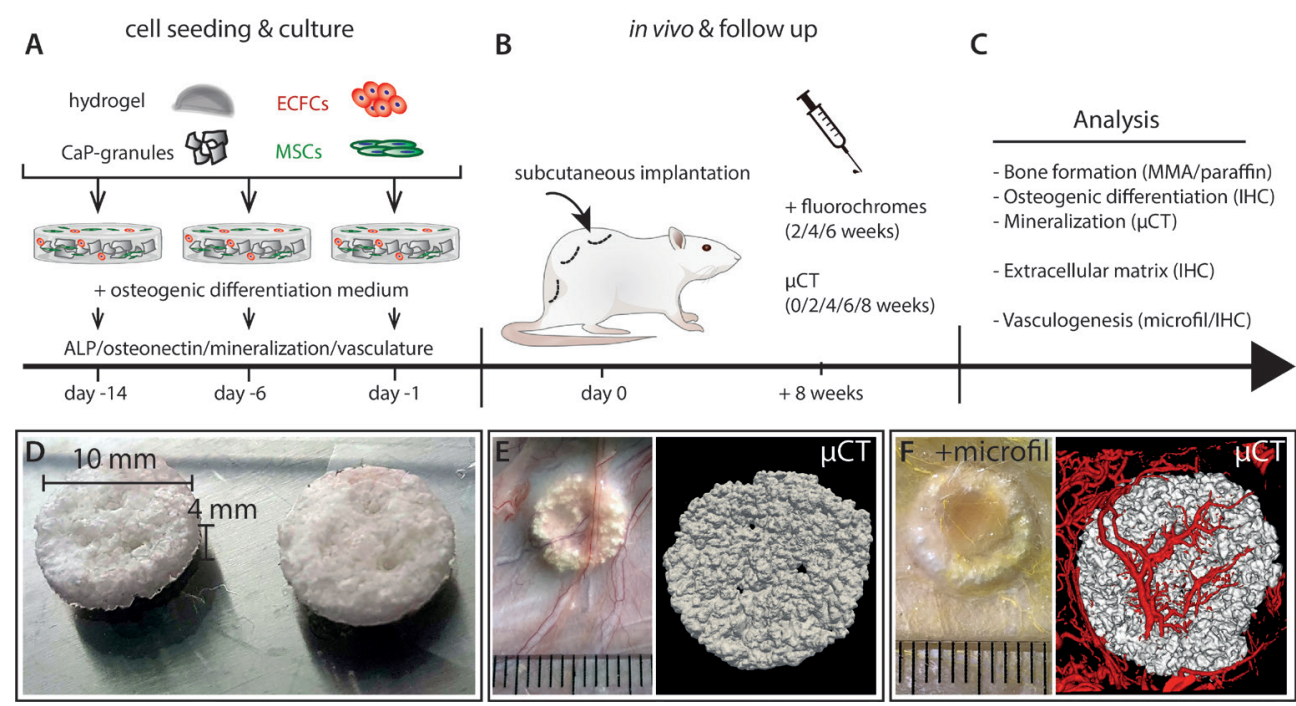

Figure 5.1. Experimental outline. A) Endothelial colony forming cells (ECFCs) and multipotent mesenchymal stromal cells (MSCs) were encapsulated in gelatin-PEG hydrogels containing calcium phosphate (CaP) granules. These constructs were cultured for different periods in an osteogenically stimulating environment in vitro and checked for their osteogenic differentiation and level of vasculogenesis. B) After pre-differentiating for 14 days, 6 days or 1 day, constructs were implanted subcutaneously in immunocompromised rats for 8 weeks. Fluorochromes were injected at 2, 4 and 6 weeks and $\mu \mathrm{CT}$ scans were made to follow construct mineralization. C) After explantation, constructs were collected and processed to evaluate bone formation, to stain for ECM components and to analyze the level of vasculature. D) CaP-containing constructs were made in the dimensions of $10 \mathrm{~mm}$ in cross-section and $4 \mathrm{~mm}$ in height and E-F) were explanted 8 weeks post-implantation. The vasculature was perfused with Microfil and vascular length within the constructs was examined via $\mu \mathrm{CT}$ angiography.

\subsection{Cell culture and characterization}

\section{Endothelial colony forming cells (ECFCs) and multipotent mesenchymal stromal cells (MSCs)}

ECFCs and MSCs were isolated and characterized as described previously [206] (details in supplementary materials and methods). ECFCs in passage 5 were transduced with a lentiviral green fluorescent protein (GFP) construct as described and presented previously [103] (details in supplementary materials and methods). ECFCs were then cultured in complete endothelial growth medium-2 (EGM-2) containing Endothelial Basal Medium-2 + SingleQuots (Lonza), $100 \mathrm{U} / \mathrm{mL}$ penicillin and $100 \mu \mathrm{g} / \mathrm{mL}$ streptomycin (PenStrep, Gibco), and 10\% heat-inactivated Fetal Bovine Serum (FBS-HI; Biowest S14068S1810). ECFCs were used for construct encapsulation at passages ranging from 11 to 13 (in vitro samples) or passage 11 (in vivo implantation). MSCs were isolated and expanded in MSC expansion medium, consisting of a-Minimum Essential Medium (Gibco Paisley, 22561), supplemented with $10 \%$ FBS, $100 \mathrm{U} / \mathrm{mL}-100 \mu \mathrm{g} / \mathrm{mL}$ PenStrep, $0.2 \mathrm{mM}$ L-ascorbic acid-2phosphate (ASAP, Sigma), and $1 \mathrm{ng} / \mathrm{mL}$ basic fibroblast growth factor (rh-FGF-2; R\&D 
Systems). The MSCs were encapsulated in the construct at passage 4 (in vitro analysis) and passage 3 (in vivo implantation).

\subsection{Gelatin-PEG co-cultures with CaP granules in vitro}

\subsubsection{CaP granule preparation}

35/65\% HA/ 3 TCP CaP granules (250-1000 $\mu \mathrm{m})$ were obtained from Kuros Biosciences and incubated in EGM-2 medium overnight in a humidified incubator at $37^{\circ} \mathrm{C}$. Medium was aspirated and granules were left to dry at $37^{\circ} \mathrm{C}$ for $1-2$ hours before encapsulating in gelatin-PEG hydrogels.

\subsubsection{Gelatin-PEG hydrogel preparation}

Hydrogels were fabricated as described elsewhere [294]. In brief, gelatin (beMatrixTM LS-H high bloom, Nitta Gelatin NA Inc, endotoxin count $<10$ endotoxin units (EU)/g) and transglutaminase modified PEG (PEG-GIn) were covalently crosslinked via an enzymatic reaction with coagulation factor XIII (Cluvot $250 \mathrm{IE}, \mathrm{CSL}$ Behring). The PEG-GIn was synthesized by conjugation of an eight-arm PEG-vinyl sulfone (8-PEG-VS, mol wt $40 \mathrm{kDa}$ ) to the specific substrate sequence (H-NQEQVSPL-ERCG-NH ${ }_{2}, \mathrm{TG}-\mathrm{Gln}$ ), facilitating crosslinking of the PEG-glutamine sequence to the native lysine residues on gelatin. Factor XIII (200 U/ $\mathrm{mL}$ ) was first activated with $20 \mathrm{U} / \mathrm{mL}$ thrombin (Baxter) in the presence of $2.5 \mathrm{mM} \mathrm{CaCl}_{2}$ for 15 min at $37{ }^{\circ} \mathrm{C}$, and was stored at $-80^{\circ} \mathrm{C}$ until use (FXIIla). Gelatin-PEG (gelPEG) $(4: 1,2 \%$ $\mathrm{w} / \mathrm{v}$ ) hydrogels were formulated in tris-buffered saline (TBS, $\mathrm{pH} 7.6,40 \mathrm{mM}$ ) with $50 \mathrm{mM}$ calcium chloride and hydrogel crosslinking was initiated upon addition of $10 \mathrm{U} / \mathrm{mL}$ factor XIIIa.

\subsubsection{In vitro co-cultures in gelatin-PEG hydrogels}

Embedding MSC/ECFC/CaP in gelPEG: Hydrogel droplets $(75 \mu \mathrm{L})$ were made for the in vitro screening of the pre-culture period. MSCs and ECFCs were trypsinized and coencapsulated at a concentration of $5 \times 10^{6}$ and $1.25 \times 10^{6}$ cells per $\mathrm{mL}$ in the hydrogel formulation, respectively. Droplets of $75 \mu \mathrm{L}$ were placed at the center of a well in a prewarmed 12-wells plate. CaP granules $(\sim 14 \mathrm{mg})$ were added per hydrogel droplet and constructs were left to crosslink for 1 hour in a humidified incubator $37^{\circ} \mathrm{C}$.

In vitro culturing of $\mathrm{CaP}$ constructs: constructs were cultured in freshly prepared osteogenic differentiation medium (ODM), composed of MSC expansion medium supplemented with $10 \mathrm{mM} \beta$-glycerophosphate (Sigma), and $10 \mathrm{nM}$ dexamethasone (Sigma). To check for mature osteoblast cell function and monitor the status of mineralization over time, $20 \mu \mathrm{M}$ xylenol orange (XO) tetrasodium salt (33825; Sigma-Aldrich) was added to the medium. Medium was replaced 2 times a week and constructs were cultured for 3, 7, 14 (total of $\mathrm{N}=6$ independent experiments with 2 MSC donors, $\mathrm{n}=3$ in triplicates) or 21 days $(\mathrm{N}=3$, $\mathrm{n}=2$ ). 
Monitoring of differentiation: signs of pre-vascular network formation (ECFC-GFP), calcium deposition (XO, RFP) and matrix mineralization (bright field and XO, RFP) were monitored every day on an Evos FL cell imaging system (ThermoFisher). Afterwards, constructs were fixed with formalin (10\%) and cut in pieces for ALP staining, paraffin embedding and whole mount immunofluorescent confocal imaging (Leica SP8x).

\subsection{In vitro osteogenic differentiation}

\subsubsection{Alkaline phosphatase (ALP)}

ALP was used to indicate early osteogenesis, as its activity is associated with committed osteoprogenitor cells. For detection of ALP activity, samples were stained within 7 days after fixation by first washing $3 \times 5$ minutes in Tris-buffered saline (TBS), followed by 15 minutes of permeabilization with $0.1 \%$ triton in TBS with subsequent washing steps of $3 \times 5$ minutes. ALP substrate (K062511, Fuchsin substrate-chromogen system, Dako) was prepared according to the manufacturer's protocol and incubated for 1 hour on the samples. To stop the reaction, samples were washed in Milli-Q (MQ) water and immediately used for imaging on a stereo microscope (Olympus SZ61).

\subsubsection{Osteonectin (ON)}

Osteogenic differentiation after 3,7 and 14 days was determined via osteonectin (ON) immunohistochemistry. Before paraffin embedding, samples were decalcified for 3 days in $10 \%$ ethylenediaminetetraacetic acid (EDTA)-PBS solution (pH 7.4), dehydrated in a graded ethanol series (70-100 \%) and transferred to xylene. Subsequently, dehydrated samples were embedded in paraffin-wax and sectioned into $5 \mu \mathrm{m}$-thick slices on a microtome. Slides were deparaffinized with xylene and gradually rehydrated through an ethanol series (100- $70 \%$ ) to demi water before starting the IHC staining (details of staining in supplementary Table 1).

Peroxidase blocking ( $10 \mathrm{~min}$ in $0.3 \% \mathrm{H}_{2} \mathrm{O}_{2}$ ) was performed before antigen retrieval. After protein blocking (15 min, 5\% BSA/PBS), slides were incubated with the primary anti-ON antibody (DSHB, AON-1 was deposited to the DSHB by Termine, J.D. (DSHB Hybridoma Product $A O N-1)$ ) in $5 \% \mathrm{PBS} / \mathrm{BSA}$ and incubated at $4^{\circ} \mathrm{C}$ overnight. Sections were washed $3 x$ for 5 min with PBS-Tween, and incubated with an anti-mouse horseradish peroxidase (HRP)-labelled polymer for 30 minutes at room temperature (RT). Addition of 3,3'diaminobenzidine (DAB) substrate solution (SK4100, Vector Laboratories) to the HRP antibodies resulted in a brown staining. Nuclei were counterstained in blue with Mayer's hematoxylin (Merck). Finally, the sections were dehydrated and mounted with DEPEX. 


\subsection{Gelatin-PEG co-cultures with granules in vivo}

\subsubsection{Preparation of gelatin-PEG co-cultures with granules}

The outcomes of the small hydrogels (section 3.3.3) were used to base the required pre-differentiation time for the up-scaled in vivo constructs on. Constructs for in vivo implantation were made using custom-made cylindrical shaped silicon molds (SYLGARD silicone elastomer kit, Sigma) (10 mm $\varnothing, 4 \mathrm{~mm}$ height) with 3 needles ( $21 \mathrm{G}, 0.8 \mathrm{~mm}$ ) placed upright for channel fabrication to promote diffusion during culturing. Silicon molds were sterilized by submerging in $70 \% \mathrm{EtOH}$ and left to evaporate completely overnight, followed by UV sterilization. The CaP granules were measured ( $0.1 \mathrm{cc}, \sim 1 \mathrm{~g}$ per construct) and incubated in EGM-2 medium overnight in a humidified incubator at $37^{\circ} \mathrm{C}$. Next, medium was aspirated and granules were left to dry. GelPEG and cells (MSC1 and GFPECFCs) $(150 \mu \mathrm{L})$ were prepared as described in section 3.2.2 and $75 \mu \mathrm{L}$ was pipetted into the mold, after which the CaP granules were added to the hydrogel layer, and the second half of the hydrogel mixture was pipetted onto the construct. CaP-hydrogel constructs were left to crosslink for 1 hour and thereafter gently released from the mold and placed in a 12-wells plate in ODM. Medium was refreshed after 1 and 2 hours, the next day and hereafter every 2 days. Samples were implanted after one day (T-1), 6 days (T-6), or 14 days (T-14). Constructs without cells (control) were kept in ODM for 6 days before implantation.

\subsubsection{Subcutaneous implantation}

The study was carried out in 14 male nude rats (Crl:NIH-Foxn $7^{\text {rnu }}$ Charles River) that were housed in triplets in the animal facility of the University Medical Center Utrecht. Animal procedures were approved by the animal ethical committee; animals received standard food pellets and water ad libitum and were kept under climate-controlled conditions (21 ${ }^{\circ} \mathrm{C} ; 12 \mathrm{~h}$ light/12 h darkness) in sterile filter-top cages. At the age of 12 weeks, after at least 1 week of acclimatization in the animal facility, subcutaneous pockets were created and samples were implanted. Surgical procedures were performed aseptic under general anesthesia (1-3.5 \% isoflurane). In total, 6 isolated implantation sites were made per animal by means of an incision and creation of a pocket with blunt scissors. After construct implantation, the fascia and skin were closed using Vicryl rapide 4-0 (VR 2297; Ethicon). Subcutaneous injection of pain medication (carprofen, $0.05 \mathrm{mg} / \mathrm{kg}$ body weight) was given pre-operatively and once a day for the following 3 days, during which the animals were also checked for complications and behavior. Per experimental group (T-1, T-6 and $\mathrm{T}$-14) $\mathrm{n}=11$ samples were implanted in randomized pockets and CaP/hydrogels without cells served as controls $(n=3)$. One rat did not recover from the anesthesia and one rat had to be euthanized after 6 weeks (non-implant related), resulting in T-1 n=9, T-6 n=10, T-14 $\mathrm{n}=9$ and control $\mathrm{n}=3$ samples after 8 weeks. 


\subsubsection{Gene expression of in vitro cultures}

Part of the samples prepared together with the ones aimed for the in vivo implantation were used for in vitro gene expression analysis one day after the surgery $(n=6 T-2, n=7$ for the samples of T-7 and T-15); a more detailed description can be found in the Supplementary materials and methods and Supplemenatary Table 2. Gene expression was evaluated for markers associated with vasculogenesis (PECAM1 (CD31), ACTA2 (aSMA) and CLAP (NG2)) and osteogenesis (ALPL (ALP), RUNX2, SPP1 (osteopontin), BGLAP (osteocalcin), COL1A1 (Collagen type I). Cycle threshold (Ct) values were normalized for the housekeeping gene $(\Delta C \mathrm{t})$ and the relative gene expression was calculated according to the $2^{-\Delta C t}$ method [295]. To test for differences in gene expression activity between the different groups (T-2, T-8 and $\mathrm{T}-15)$, the transformed normalized gene expressions $\left(2^{\Delta C T}\right)$ of the samples were used.

\subsection{In vivo outcome parameters}

\subsubsection{Bone formation - fluorochromes}

To estimate the timing of the onset of in vivo bone formation, fluorescent calcium-binding fluorochromes that deposit at the site of active mineralization were used. Three different colors were used to give sequential information regarding the start and direction of bone formation. After 2 weeks, $100 \mathrm{mg} / \mathrm{kg}$ xylenol orange tetrasodium salt (XO, red) (33825, Sigma-Aldrich, $20 \mathrm{~g} / \mathrm{L}$ in $\mathrm{NaHCO}_{3}$ 1\%) was injected. At 4 weeks, $10 \mathrm{mg} / \mathrm{kg}$ calcein green (C0875, Sigma-Aldrich; $3 \mathrm{~g} / \mathrm{L}$ in $\mathrm{NaHCO}_{3}$ 2\%) was injected and after 6 weeks, tetracycline hydrochloride (yellow) $20 \mathrm{mg} / \mathrm{kg}$ (T3383, Sigma-Aldrich; $2 \mathrm{~g} / \mathrm{L}$ in 50\% demi/PBS) was injected subcutaneously.

\subsubsection{Bone formation - embedding in methylmethacrylate (MMA)}

After explanting, non-decalcified samples T-1 $(n=3), T-6(n=4), T-14(n=3)$ and control $(n=1)$ were embedded in methylmethacrylate (MMA) solution (S7351990702, Merck) with the following protocol. After fixation, the samples were dehydrated through a graded ethanol series and transferred to MMA. Hereafter, samples were embedded in MMA solution with Plastoid ${ }^{\circ} \mathrm{N}$ (74432, Sigma-Aldrich Chemie $\mathrm{GmbH}$, Steinheim, Germany) and benzoyl peroxide (Sigma-Aldrich, St Louis, MO, USA) (0.8 mL MMA, 0.2 mL Plastoid ${ }^{\circ} \mathrm{N}$ and $28 \mathrm{mg}$ benzoyl peroxide). Cross-sectional slides in the Z-direction (20-30 $\mu \mathrm{m}$ ) were made using a saw microtome (Leica SP1600; Leica Biosystems Nussloch GmbH, Nussloch, Germany). For histopathology, the sections were stained with $1 \%$ methylene blue solution and subsequently with $0.3 \%$ basic fuchsin solution. Slides were alternated between the fuchsin staining and unstained sections to visualize the fluorochrome labels.

\subsubsection{Vascular network - Microfil}

Vascularization was examined after 8 weeks with the use of $\mu C T$ angiography. Under general anesthesia (1-3.5 \% isoflurane in oxygen), $500 \mathrm{U}$ heparin were injected intravenously, the diaphragm was cut and a catheter was placed in the left ventricle 
and connected to a roller pump. Next, $150 \mathrm{~mL}$ of PBS, then $50 \mathrm{~mL}$ of $0.4 \%$ papaverine solution (P3510; Sigma-Aldrich), followed by $250 \mathrm{~mL}$ of formalin was perfused through the animal. After injection of $20 \mathrm{~mL}$ radiopaque contrast agent (Microfil MV-120, Flow Tech) the compound was polymerized overnight at $4{ }^{\circ} \mathrm{C}$. The next day, constructs were explanted and a $\mu \mathrm{CT}$ scan was performed of the individual samples (scan time: $3 \mathrm{~min}$; voxel size: $20 \mu \mathrm{m}^{3}$; X-ray energy and X-ray tube potential: $90 \mathrm{keV}$; X-ray intensity: 180 $\mu A$; Quantum FX, PerkinElmer). Hereafter, $n=6$ samples per group (control group $n=2$ ) were decalcified in $10 \%$ EDTA-PBS solution ( $\mathrm{pH} 7.4$ ) for 4 weeks. After decalcification, the constructs were placed back in the $\mu \mathrm{CT}$ and the volume of the polymerized vessels within the ghost of the construct was measured. Volumes of interest (VOI) were segmented with a global threshold and inverted. Vessel volume was reconstructed and measured in $\mathrm{mm}^{3}$ with the ImageJ plugin BoneJ [296] and the ROI manager function. Three-dimensional (3D) reconstructions of the defects were made using image processing software (Fiji). The decalcified construct were then embedded in paraffin for histological evaluation.

\subsubsection{Formation of bone and vasculature - paraffin embedding}

Decalcified samples were dehydrated in graded ethanol series, paraffinized and sectioned into $5 \mu \mathrm{m}$-thick slices on a microtome. Hematoxylin and eosin (H\&E) staining was used to visualize (and quantify) new bone formation and to get a general overview of the sample. Bone tissue: Osteogenic differentiation was observed via the ON staining as described in section 3.4.2 (reacts with human and rat cells). To review the origin of the newly formed bone, an anti-human staining (anti-mitochondrial staining), as well as anti-rat collagen I and anti-human collagen I were performed (details in Supplementary Table 1). After peroxidase blocking, antigen retrieval was performed and samples were incubated with the primary antibody overnight at $4^{\circ} \mathrm{C}$. DAB substrate solution was developed into a brown staining via the HRP antibodies.

Vasculature: CD34 staining (rat specific) was performed to identify endothelial cells and vessel structures derived from rat cells, a CD31 (human specific) and anti-GFP staining were carried out to visualize contribution of human ECs to the vascular network (details in Supplementary Table 1). Briefly, for the CD34 staining, after blocking endogenous peroxidase activity, antigen retrieval was performed and samples were blocked with $10 \%$ normal rabbit serum in 1\% PBS-BSA for 30 min at RT. The CD34 antibody in 1\% PBS-BSA was incubated overnight at $4^{\circ} \mathrm{C}$ and staining was visualized with $\mathrm{DAB}$ as substrate for the HRP antibodies.

All sections were visualized using an Olympus BX51 microscope (Olympus DP73 camera). The IHC staining served to confirm the origin of the newly formed tissue and to assess the different types of cells and tissues present, as well as for quantitative analyses. 


\subsubsection{Quantification of immunohistochemical staining}

The amount of positive signal from the anti-human cell staining, the $\mathrm{ON}^{+}$cells and the CD34+ structures was quantified using Adobe Photoshop CS6. For each sample, 20 images were taken from one slide to represent the whole sample. Images were loaded in Adobe Photoshop and the positive signal was selected with the color range command, with separate thresholds for the different staining (i.e., human cells, ON, CD34). The total area of the selections (in pixels ${ }^{2}$ ) was hereafter measured with the measurement tool, and the average of the 20 images represent the signal per sample.

\subsubsection{Bone formation - histomorphometry of MMA and paraffin slides}

The amount of bone formation was quantified in both the MMA samples (cross section from Z-direction) and the paraffin H\&E samples (cross section from Y-direction). At first, an overview of the whole sample was made by merging images (1.25x/0.04 FN26.5 objective) into a panoramic image in Adobe Photoshop CS6. This was done for 6 slides (MMA) or 2 slides (paraffin) throughout the depth of each sample. In case of the MMA samples, panoramic views were loaded in Adobe Photoshop CS6 and the granules, bone tissue (b) and osteoid (o) were individually selected and pseudo-colored. The amount of pixels per different structure was then quantified via 'recording measurement'. The amount of bone (b), osteoid (o) or both (b+o) ('bony matrix') was then presented compared to the quantified surface of the granules. In case of paraffin samples, panoramic views were loaded in Adobe Photoshop CS6 and all bone tissue $(b+o)$ was selected, since with the H\&E staining the osteoid was not clearly distinguishable from the bone tissue. Also, the granules were not clearly visible due to the decalcification, therefore the bone tissue could only be compared to the total construct size. The MMA samples and paraffin samples showing the $b+o$ compared to the total construct size were merged into one graph. Available space of bone formation displays the total construct volume minus the granule volume of the MMA samples.

\subsection{Statistical analysis}

Statistical analyses were performed with GraphPad Prism 6.01. The in vitro gene expression at T-2, T-8 and T-15 were tested for significant differences by using a one-way ANOVA with a post-hoc Tukey test or non-parametric Kruskal-Wallis test in case of non-normal distribution. Histogram plots show the transformed $\left(2^{-\Delta c t}\right)$ values and outliers based on the $2^{-\Delta c t}$ values (identified using the ROUT test $(\mathrm{Q}, 1 \%)$ ) are indicated with a $(x)$ sign. The histogram includes the median, and the mean is indicated with the $(+)$ sign.

Analysis for bone formation based on the MMA and paraffin samples were performed by comparing the averaged percentage of bony matrix, bone or osteoid per sample $(n=6$ slides/sample for MMA, $n=2$ slides/sample for paraffin) between the groups T-1, T-6 and T-14 with a one-way ANOVA (post-hoc Tukey). 
The area $\left(\right.$ pixel $\left.^{2}\right)$ of the anti-human mitochondria staining and the osteonectin and CD34 marker were tested for significant differences between the mean pixel values ( $n=20$ images/slide) of the bone forming samples of the T-1, T-6 and T-14 groups. First, outliers within the $n=20$ images per sample were identified using the ROUT test $(Q, 1 \%)$ and excluded from the statistical testing. Using a one-way ANOVA, differences between the experimental groups were considered statistically significant when $p<0.05$ and represented as mean \pm standard deviation.

\section{Results}

\subsection{Co-cultured CaP constructs show osteogenic differentiation in vitro}

To screen for the onset of osteogenic differentiation in the CaP-containing co-cultures, hydrogel constructs $(75 \mu \mathrm{L})$ were made and used to determine the required predifferentiation period for the up-scaled in vivo constructs.

The onset of in vitro osteogenic differentiation in the MSC-ECFC co-cultures was verified with a staining for ALP. The ALP activity increased with prolonging pre-culture periods in ODM in the small hydrogel constructs (Supplementary Figure 5.1 A) and the up-scaled precultured in vivo constructs (Figure 5.2 A), with the highest activity reached after 14 days. After 21 days, the ALP activity was localized to the vicinity of CaP granules (Supplementary Figure 5.1 A) and declined towards the periphery. Moreover, differentiation of MSCs towards osteoblasts was further verified by ON expression, which indicated their transition to more mature osteoprogenitor cells. An increase of ON-positive cells was observed, mostly between day 3 and day 7 of culture, especially around the CaP granules and was found at a similar intensity after 14 days in the small hydrogel samples, but now throughout the whole gel (Figure 5.2 B).

Next, to verify the progression of osteogenic differentiation towards maturation of the matrix, the fluorochrome xylenol orange $(\mathrm{XO})$ was added to the culture medium to be able to assess mature osteoblast function and monitor the matrix mineralization. Clear calcium deposition at a cellular level became apparent at day 14 with specific localization around the granule site (Figure 5.2 C) and was further increased at day 21 (Supplementary Figure 5.1 B-C), indicating mature osteoblast function. At day 21 , matrix mineralization could also be detected by the dark appearance on brightfield microscopic images (Supplementary Figure 5.1 B) and $\mathrm{XO}$-positive $\mathrm{CaP}$ deposition in the matrix (Supplementary Figure 5.1 C-E). Besides the osteogenic activity of the cells on protein level, their commitment was also confirmed via gene expression analysis (Figure 5.2 D). After 2, 7 and 15 days of differentiation, all the constructs showed gene expression of the analyzed osteogenic genes ALP, SPP1, RUNX2, BGLAP and the gene for collagen type I, which is the template on which mineralization occurs. 


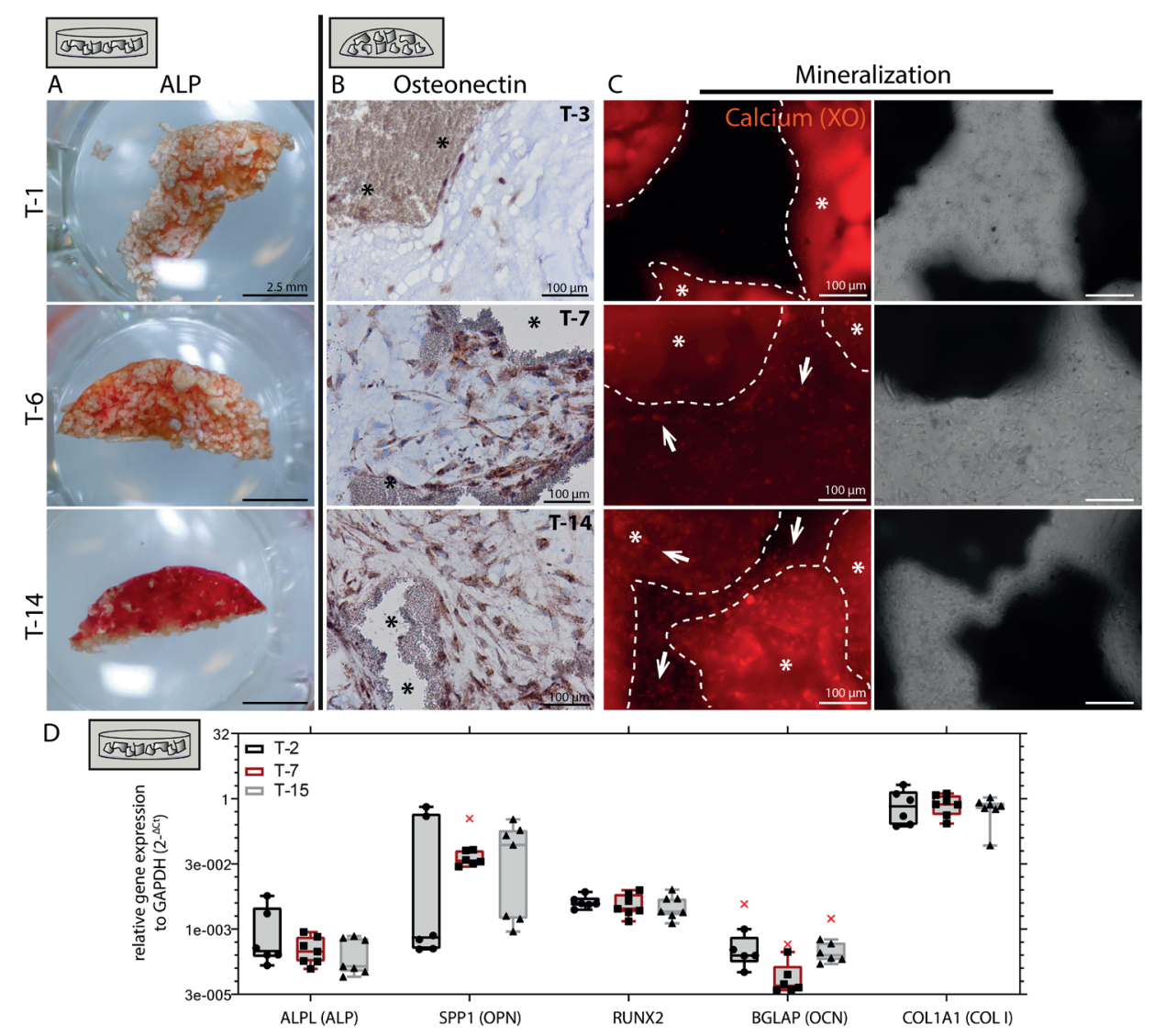

Figure 5.2. In vitro osteogenic differentiation of cell-laden CaPgelatin-PEG hydrogels. A) Representative images of ALP activity (red) in cylindrical in vivo up-scaled constructs at the time points of in vivo implantation. The intensity of the ALP activity increased over time. B) Osteonectin protein expression (brown staining) was visualized by immunohistological staining after 3, 7 or 14 days of culture in small hydrogel droplets (* represent ghost of the CaP granules). C) Calcium depositions were visualized with xylenol orange (XO, in red) (white arrows) during the time of culture. The granules are lined with the white dotted line. Bright field images (right row) represent the same location and visualize the granules (dark signal). D) Boxplots representing the $2^{-\Delta C t}$ relative gene expression after $3(n=6), 7(n=7)$ or $15(n=7)$ days of osteogenic differentiation of the in vivo cylindrical constructs, normalized to the GAPDH housekeeping gene. All constructs showed comparable gene expression of the selected osteogenic genes, $\left({ }^{*} p<0.05\right)$. Boxplots represent the median without the outliers $(x)$.

\subsection{The in vitro vasculogenic network is influenced by CaP granules}

Evaluation of the vascular network in the constructs showed that at 5 days after initiation of the CaP co-culture, a pre-vascular network had developed in the $75 \mu \mathrm{L}$ co-culture model (Figure 5.3 A-C). a-SMA-positive cells were supporting the pre-vascular structures (Figure 5.3 C), as well as attaching to the granules (dotted line). Remarkably, a regression of the vascular 
network was observed after 10 days of culturing (Figure $5.3 \mathrm{D}$, same location as A-B) where the vascular structures specifically deteriorated near the granules (Figure $5.3 \mathrm{~F}$ ). Coverage of the granules by mural-like a-SMA-positive cells remained intact but were characterized by a disorganized structure around the vessel remnants (Figure 5.3 F-G, white arrows). The regressed vascular structures showed a morphology similar to that of apoptotic cells, with fragmented nuclei and assumed apoptotic bodies or accumulation of (GFP) protein in intracellular microvesicles (Figure $5.3 \mathrm{H}$ ) whereas the nuclei of non-endothelial cells did not show fragmentation (Figure $5.3 \mathrm{H}$, small arrows). The up-scaled construct type for the in vivo implantation followed the same trend in terms of vascular development and degradation. qPCR analysis $\left(2^{-\Delta C t}\right)$ showed that the selected vasculogenic genes were expressed in all of the implanted samples, independent of the culture time. Only CD31 was expressed higher in the T-15 samples when compared to samples cultured for 2 days ( $p=0.027$ ), in contrast to the localized decrease of vascular structures shown in Figure 5.3 D-H. The gene expression of the mural markers a-SMA and NG2 remained constant over the period of pre-differentiation.It is important to note that the degradation of the vascular network in vitro was mainly apparent in the T-14 construct, indicating that in this group the pre-vasculature was absent at the time of in vivo implantation.
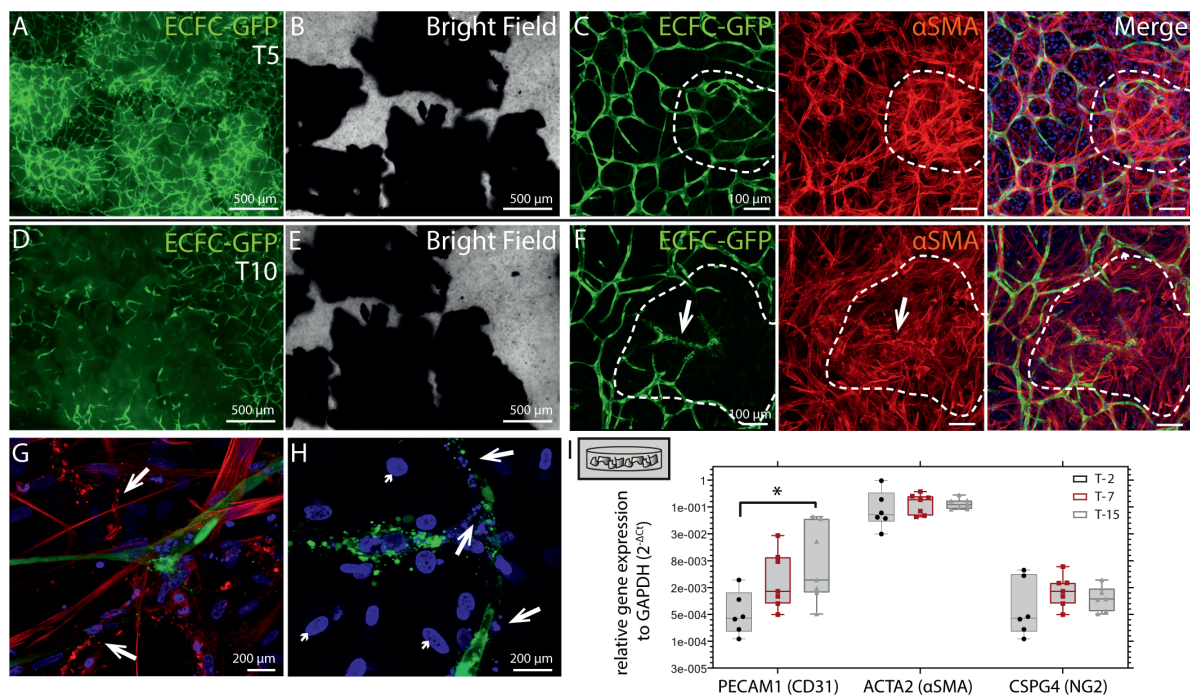

Figure 5.3. In vitro pre-vascularization of cell-laden CaP gelatin-PEG hydrogels. A) The pre-vascular network was evaluated via the GFP-ECFC signal (green) in the small hydrogel droplets $(75 \mu \mathrm{l})$ after 5 days. B) Dark structures in the bright field image represent the granules. C) The pre-vascular structures were supported by a-SMA positive cells (red), which also attached to the granules (dotted white line). D) Later, the pre-vascular network regressed in the vicinity of the granules (D-E contains the same location as A-B). F) The a-SMA-positive cells that were attached to the granules remained intact but were $G$ ) disorganized around the regressed vessels (white arrow). $H$ ) The nuclei (DAPI, blue) in the vessel remnants were fragmented (white arrows), whereas the surrounding nuclei showed a normal appearance (small white arrows). I) Boxplot representing the relative gene expression ( $\left.2^{-\Delta C t}\right)$ after $3(n=6), 7(n=7)$ or $15(n=7)$ days of osteogenic differentiation of the in vivo cylindrical constructs, normalized to the GAPDH housekeeping gene. All constructs showed gene expression of the selected vasculogenic associated genes, only CD31 was significantly upregulated at day 15 compared to day $2\left(^{*} p<0.05\right)$. Central line in the boxplots represents the median. 


\subsection{The prolonged pre-differentiation period accelerated the onset of in vivo bone formation}

After the 8-week implantation period of the T-1, T-6 and T-14 groups, all samples were successfully retrieved from the subcutaneous pockets. The samples better maintained their shape with increasing pre-culture period (T-6 and T-14) (Supplementary Figure 5.2 A-D). T-1 and control (no cells) samples showed deformation and some displacement of the granules, but these remained contained within the pocket area.

Methylene blue/basic fuchsin-stained MMA sections and H\&E-stained paraffin sections were evaluated for bone formation and tissue morphology. The control group (no cells) did not show any bone formation (Supplementary Figure 5.2 A). Overall, bone was observed in 1 out of 9 samples for the T-1 group (0/3 MMA and 1/6 paraffin), 7 out of 10 for the T- 6 group (3/4 MMA and 4/6 paraffin) and in 6 out of 9 for the T-14 group (2/3 MMA, 4/6 paraffin). Additional, construct mineralization was quantified over time by $\mu C T$ scanning at $0,2,4,6$ and 8 weeks and is described in Supplementary materials and methods, Supplementary results and Supplementary Figure $5.2 \mathrm{H}$.

Of the T-1 samples, 1 of the 3 MMA-embedded samples contained limited osteoid formation in one location (Figure 5.4 B, black arrows), but mostly showed fibrous tissue ingrowth and a high occurrence of multi-nucleated giant cells (MGCs) at the edges of the granules (Figure 5.4 C, ${ }^{*}$ granules, \#MGCs). The other two MMA embedded samples did not show any bone formation. Samples belonging to the T- 6 group revealed a high prevalence of osteoid (Figure 5.4 D-E, black arrows) compared to bone (Figure 5.4 D-E, white arrows). Bone marrow cavities were observed in the samples with bone formation (Figure $5.4 \mathrm{E}, \mathrm{BM}$ ) and osteoblasts were lining the bone tissue (Figure $5.4 \mathrm{~F}, \wedge$ ). T-14 samples formed bone with a higher prevalence of bone versus osteoid (Figure $5.4 \mathrm{G}$ ). Also here, bone marrow cavities were observed (Figure $5.4 \mathrm{H}$ ) and cubical-shaped osteoblasts were actively secreting new osteoid on top of more mature bone (Figure $5.4 \mathrm{I}$ ). Some of the bone-forming samples, independent of pre-culture time, showed mineralized cartilage tissue within the bone (Supplementary Figure 5.3, \#), which was also positive for glycosaminoglycans (GAGs) and had a disorganized and loose collagen structure $(\wedge)$.

The amount of bone tissue was quantified by carrying out histomorphometry on the stained MMA and paraffin sections. No significant differences were detected in the amount of osteoid tissue and/or bone tissue between the three time points (Figure $5.4 \mathrm{~J}$ ). When pooling the histomorphometrical data (Figure $5.4 \mathrm{~K}$ ) from both the paraffin samples and MMA samples, a significant difference in the total amount of osteogenic tissue (bone tissue and osteoid combined) between the T-1 $(0.94 \% \pm 2.63)$ and T-14 $(7.17 \% \pm 5.96)$ samples ( $p=0.039$ ) was shown. However it should be noted again that the T-1 group only 
contained 1 sample that formed bone. The total area available for bone formation was $65.29 \% \pm 4.16$ (Figure $5.4 \mathrm{~K}$, dotted line) (materials and method section 3.6.6).

Next, we investigated if the initiation of bone tissue formation varied between the different periods of pre-differentiation. For this, we sequentially injected fluorochromes at 2, 4 and 6 weeks after implantation. The MMA histological sections from the T-1 group showed no fluorochrome signal in the samples, in agreement with the observed lack of bone formation (Supplementary Figure 5.2 E). The majority of the bony tissue in the T- 6 group did not contain any fluorescent signal, indicating either an absence of mineralization (thus indicating that the construct consisted mostly out of osteoid tissue) or that the mineralization of the osteoid started after the last fluorochrome (week 6, tetracycline, yellow) was cleared from the body (Supplementary Figure 5.2 F). This was plausible as the basic fuchsin-stained sections had a predominant presence of osteoid tissue in T-6 samples at these locations. The areas that did contain fluorochrome signal in the T-6 samples, showed that in some areas the bone formation was initiated at week 4 (calcein, green) and was still ongoing by week 6 (tetracycline, yellow), with the mineralizing front moving away from the CaP material. However, most of the bone was formed after 6 weeks, indicated by the presence of tetracycline-positive bone tissue that was directly deposited onto the granules with a layer of non-fluorochrome stained bony tissue on top of the signal (Figure $5.4 \mathrm{~L}$ ). No active bone formation was observed at 2 weeks (XO, red). The sections from the periphery of the constructs showed more fluorochrome negative bone tissue compared to the sections of the core region, implying that the onset of bone formation occurred at the center of the constructs.

In the samples of the T-14 group, areas with a red signal (week 2, XO, red) were detected, indicating an early onset of bone formation in the T-14 group (Figure $5.4 \mathrm{M}$ ). Also, the constructs presented active bone formation at 4 weeks (calcein, green). Here, a relatively thick layer of calcein-positive bone was directly formed on top of the granules with an additional layer of newly formed bone by 6 weeks (tetracyclin, yellow) and another layer of unstained osteoid tissue on top (dotted line, white arrow), indicating the direction of the bone growth away from the granule surface (Figure $5.4 \mathrm{~N}$ ). Similar to T-6 samples, areas without any fluorochrome incorporation were found (Supplementary 5.2 F, representative image for both T-6 and T-14). In addition, many areas of bone were found with only the yellow label present, showing initiation of bone formation after 6 weeks (Supplementary Figure $5.2 \mathrm{G}$ ). 

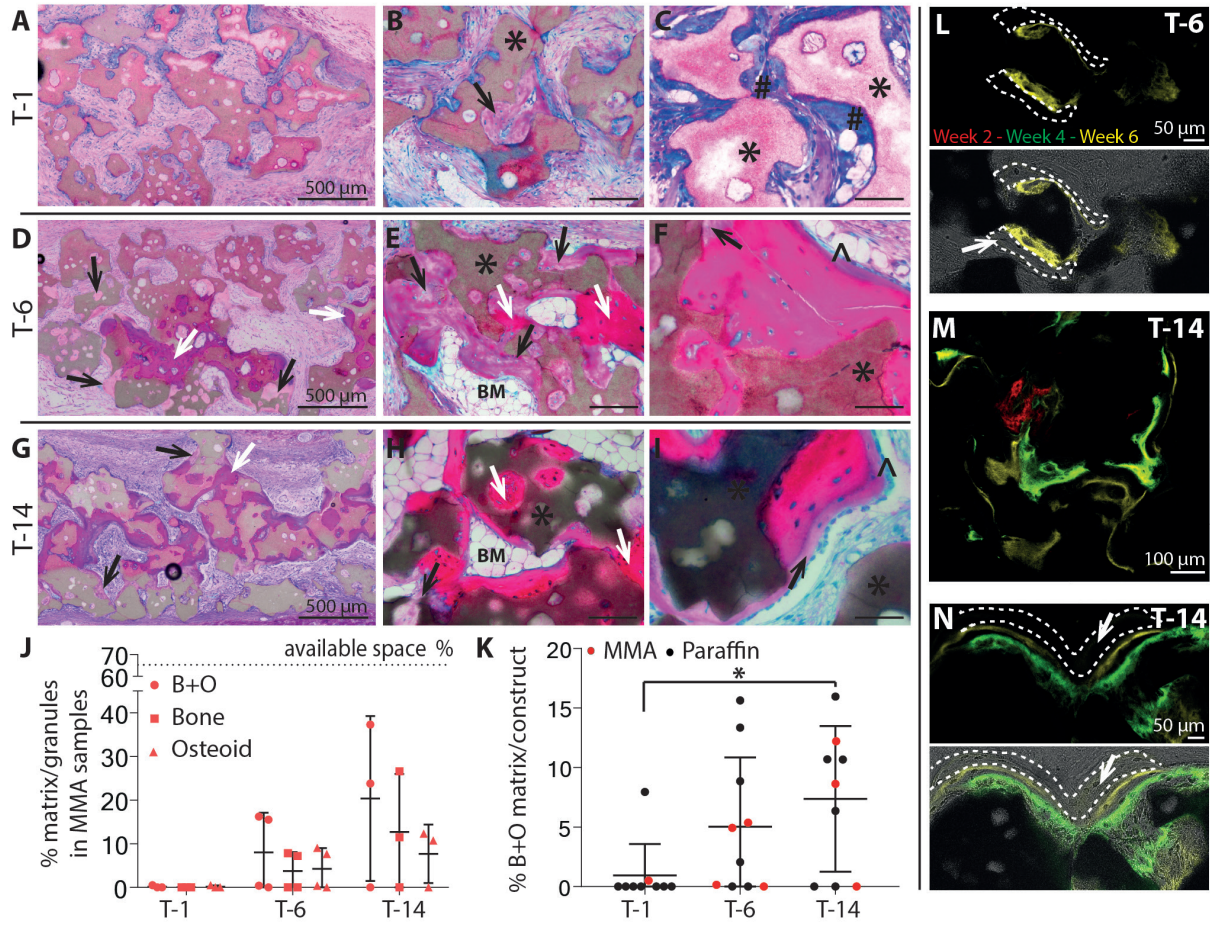

Figure 5.4. In vivo bone formation 8 weeks after implantation. Bone formation is shown with methylene blue/basic fuchsin staining on MMA slides of T-1 A-C), T-6 D-F) and T-14 G-I). B) light pink represents osteoid (black arrow) in the T-1 constructs. C) Multi-nucleated giant cells (MGCs) are represented by $(\#)$, and attached to the granules $(*)$. D) Samples pre-differentiated for 6 days showed both osteoid (black arrow) and bone (dark pink, white arrow). E) Locations with bone marrow were observed with F) osteoblasts lining the bone $(\wedge)$. G-H) Samples from the T-14 group show bone formation (dark pink, white arrows) and osteoid (light pink, black arrows). I) Osteoblasts were lining the bone, on top of an osteoid layer (black arrow). J) Histomorphometry of bone tissue in MMA sections, averaged values of 6 slides per sample, relative to the amount of granules. $K$ ) Histomorphometry of the bone+osteoid tissue of the paraffin (black dots) + MMA constructs (red dots), showed that there was a difference in total amount of bony tissue between T-1 $(0.94 \% \pm$ $2.63)$ and T-14 $(7.17 \% \pm 5.96)\left({ }^{*} p<0.05\right)$. L) Representative image of the fluorochrome sequence as found in the T- 6 group, red-green-yellow shows bone formation after 2-4-6 weeks, respectively. M) Mineralization in the T-14 group shows bone formation after 2 weeks (red), 4 weeks (green) and 6 weeks (yellow). N) Bony tissue was deposited on top of the yellow label, indicating continued bone formation after 6 weeks until week 8 , as indicated with the dotted white line and white arrow. Scale bars represent $50 \mu \mathrm{m}$ unless indicated otherwise.

\subsection{Bone formation was reliant on the retention of (human) donor cells within the construct}

The tissue sections were then evaluated their overall morphology and to determine the species origin of the bone tissue. The retention of the donor cells was evaluated via IHC staining against human anti-mitochondria on paraffin slides. Human cells were present in 
areas with bone formation, independent of the pre-culture time (Figure $5.5 \mathrm{~A}$ ), with the samples of the T-14 group showing the highest retention of human cells (Figure $5.5 \mathrm{E}, p=$ 0.021). In samples with low amounts of bony tissue, very few human cells were detected. Specificity of immunodetection of human cells was confirmed with isotype controls (Supplementary Figure 5.4 A, inset). In general, human cells remained contained within the constructs and did not grow out into the surrounding rat tissue. Quantification of the signal of the positively stained cells confirmed that the samples without bone did not contain human cells (Supplementary Figure 5.4 D).

The amount and location of osteoblasts were evaluated on osteonectin (ON)-stained slides (antibody stains rat and human osteonectin). Cells that stained positive were mainly found at the edges of the newly formed bone, most frequently in the T-14 group, and were also detected embedded within the bone (black arrows). Moreover, osteoblasts were found within the fibrous tissue and the surrounding matrix was also ON-positive (Figure 5.5 B). Samples with little bone formation, only showed ON-positive cells around the granules. Samples without any bone formation did not show strong ON staining in the cells and only some matrix staining (Supplementary Figure 5.4 B). Overall, no differences were found between the groups with regard to the amount of ON-positive signal (Figure $5.5 \mathrm{~F})$.

To determine the origin of the new bone tissue, slides were stained for Collagen type I, specific for either human or rat collagen. The bone formed in the (only) bone forming T-1 sample was mostly built upon rat collagen type I, with human bone matrix directly on top of the granules (Figure 5.5 C-D). Bone forming samples from the T- 6 group showed similar results, with bone containing both rat and human collagen type I. In general, the first layer of collagen type I on top of the CaP granules was of human origin, while rat collagen I composed the layers further away from the granules. On the other hand, in the T-14 group, the bone mostly contained human collagen type I.

Based on the overall morphology of the tissue samples observed with the H\&E staining, a high prevalence of MGCs was observed in the samples without bone formation. Here, MGCs were mostly located at the edges of the granules and infiltrated throughout the whole samples. In samples with bone formation, MGCs were mainly confined to the construct edge or to areas with no bone (Supplementary Figure 5.4 C). TRAP-positive multinucleated osteoclasts were mainly found near the granules rather than on the bone (Supplementary Figure 5.5 A-C). They were only present in very low numbers in the non-bone forming samples and showed higher prevalence in the samples with bone (Supplementary Figure 5.5 D-F). 




Figure 5.5. Samples with bone formation by host and donor cells. A) Representative images of paraffin slides from bone forming samples for the anti-human staining (brown signal). Human cells were detected at the edge of the bone, within the bone (black arrow) and in the connective tissue. B) Osteonectin-positive cells represent the osteoblasts at the edge of the bone or osteocytes within the bone (black arrow). C-D) Staining specific for rat collagen type I C) or human collagen type I (D) is shown on consecutive slides of the same location; dotted indicate corresponding locations; granules are indicated with $\left(^{*}\right)$. E) Quantification of the area occupied by human cells (in pixels ${ }^{2}$ ) in the bone-containing samples showing the retention of human cells in T-14 in particular (* $p<0.05$ ). Quantification was based on $n=20$ images/slide, for 1 slide per sample. F) The area of osteonectin-positive cells (signal in pixels ${ }^{2}$ ) was comparable between the groups. Quantification was based on $n=20$ images/slide, for 1 slide per sample. G) The amount of bone formed per sample (histomorphometry on paraffin slides) is displayed with the different sample colors corresponding with those of the same sample in the graphs of $E$ and $F$. Quantification was based on panoramic view of 2 slides from different depths. Scale bars represent $50 \mu \mathrm{m}$.

\subsection{The in vivo vascular network was not influenced by in vitro pre-differentiation}

The influence of the state of the in vitro vascular network on the in vivo level of vasculature was assessed via IHC staining. The amount of vessels within the vessel network was evaluated, as well as its origin and the overall morphology. Histological slides showed that the rat tissue surrounding the samples was highly vascularized. Throughout the whole construct, ingrowth of rat endothelial cells organized in microvascular structures 
was present, independent of the sample group (Figure 5.6 A). No human endothelial cells could be detected with anti-CD31 and anti-GFP staining (Figure 5.6 B), indicating that the vascular structures were solely from rat origin. In contrast to the in vitro vasculogenic data, the rat-derived capillaries did not seem to be inhibited by the presence of granules and were present in their close vicinity (Figure $5.6 \mathrm{C}$ ). In areas without new bone tissue, capillaries were mostly found in the connective tissue without a preference for location (edge or center of the construct) (Figure 5.6 D). Areas with bone formation showed abundant capillaries in the bone marrow and only few within the bone (Figure 5.6 E). Quantification of the area of CD34-positive structures indicated that all groups had comparable amounts of vasculature within the construct (Figure 5.6 F).

The amount of perfusable vasculature was also quantified with the radiopaque contrast agent Microfil. Before euthanization, Microfil was perfused in the rats' vascular systems, and vascular volume per construct was calculated with $\mu \mathrm{CT}$ after demineralization of the sample (Figure $5.6 \mathrm{G}-\mathrm{H}$ ). The volume of the total construct ( $\mathrm{VOI}$ ) was comparable between the groups and vessel volume did not differ among the groups (Figure 5.6 I). Histologically, Microfil could be detected in some capillaries, but not in all. 

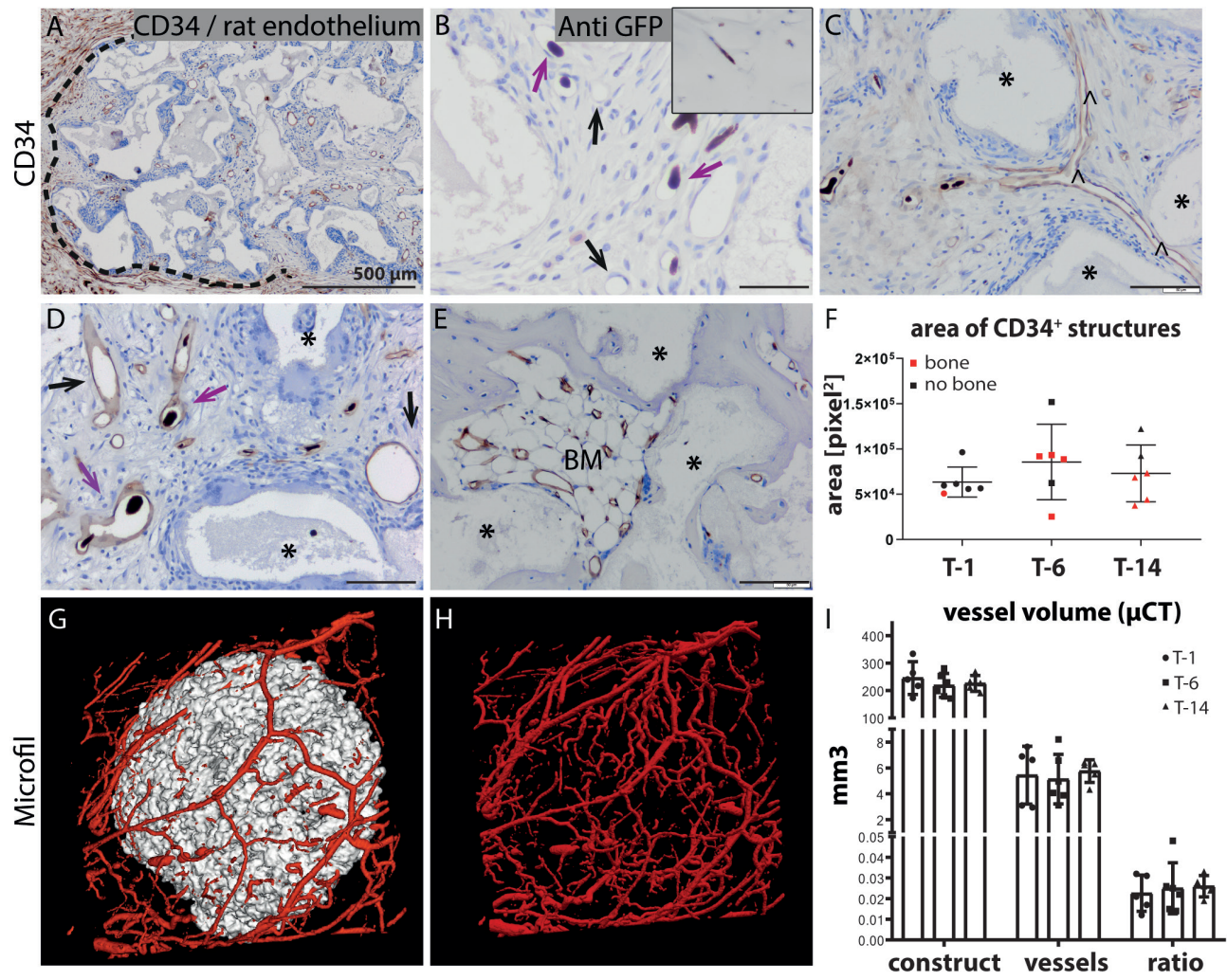

Figure 5.6. Vasculature 8 weeks after implantation. A) Cross-sectional overview of paraffinembedded samples shows the highly vascularized rat tissue (CD34, rat endothelium, brown staining) surrounding the construct and vessels within the construct (outlined by black dotted line). B) No human endothelial cells were found with the anti-GFP staining. The inset represents a positive control with human GFP-ECFCs. C) The constructs were vascularized by rat endothelium (CD34), also in close vicinity of the granules (vessel $\wedge$, granules *). D) Black arrows show CD34 ${ }^{+}$vessels, and pink arrows show vessels filled with Microfil. Vessels were mostly present in the connective tissue or E) in the bone marrow. F) Quantification of the area of CD34-positive structures (in pixels ${ }^{2}$ ) in all groups; red dots represent the samples where bone was formed; black dots represent samples where no bone formation was observed. Quantification was based on $\mathrm{n}=20$ images/slide, 1 slide per construct; $\left.\left({ }^{*} p<0.05\right) \mathrm{G}\right)$ The $\mu \mathrm{CT}$ images of Microfil-perfused animals before sample decalcification and $\mathrm{H}$ ) after decalcification were used to I) quantify the vessel volume in the ghost of the construct. The ratio of vessel volume over construct volume before decalcification was calculated. Scale bars represent $50 \mu \mathrm{m}$ unless indicated otherwise.

\section{Discussion}

This study reports on a comprehensive analysis of the in vitro and in vivo performance of an osteogenic pre-vascularized co-culture model featuring CaP granules. To do so, we integrated osteoconductive CaP granules (35/65\% HA/ $\beta$ TCP) in our previously developed osteogenic MSC/ECFC co-culture model in a gelPEG system [294]. The model provided a detailed insight in the in vitro vasculogenesis and osteogenesis in the presence of 
$\mathrm{CaP}$ ceramics, and the effect of pre-culturing on subsequent bone formation in vivo. Additionally, an earlier onset of bone formation was demonstrated with a more prolonged pre-differentiation period, as well as increased presence of the implanted cells.

Addition of osteoprogenitor cells to CaP constructs is known to enhance bone formation in vivo, and is essential for bone formation in small animal models [269-274, 292, 297]. In line with previous rodent studies, our work demonstrated that the control group (construct without cells) and the majority of the T-1 group (undifferentiated cells) did indeed fail to induce bone formation after subcutaneous implantation, and emphasized the need for a certain pre-differentiation period on top of the presence of the co-cultured cells. To gain more insight in the influence of osteogenic pre-differentiation on in vivo bone formation and vascularization, constructs with varying levels of osteogenic predifferentiation and pre-vascularization were compared. The T-1 group contained mainly undifferentiated MSCs and ECFCs, the T-6 group immature/mature osteoprogenitor cells, and the T-14 group mostly preosteoblasts/osteoblasts. These three levels of differentiation corresponded to a stage of cellular proliferation, cellular and matrix maturation, which would then be followed by matrix mineralization $[267,298]$.

To note, the T-14 group was selected to match the phase preceding the matrix mineralization. Cells from the mineralization phase normally demonstrate low self-renewal and proliferative capacity [298] and are entrapped in the mineralized matrix, meaning that they scarcely participate in deposition of new organic matrix [265]. Additionally, we postulated that a well-mineralized matrix would obstruct the influx of host-derived osteoprogenitor cells and endothelial cells, counteracting new bone formation and vascularization. For these reasons, we chose to implant constructs that were pre-cultured for up to 14 days.

Next, we progressed the given model towards more clinically relevant-sized osteogenic constructs and implanted them subcutaneously. The osteogenic pre-differentiation period did benefit in vivo bone formation and greatly increased the stability of the constructs, with active bone formation till the time of explantation. The longest pre-differentiation period resulted in larger amounts of mature bone compared to the intermediate duration of differentiation. This stresses that the prolonged differentiation can trigger an earlier initiation of bone formation upon implantation. In general, the extended predifferentiation period may have enhanced the expansion of encapsulated cells. In any case, the pre-differentiation period improved the retention of the donor cells within the construct after implantation. This retention was crucial for bone formation, since there was no bone formed in the samples in which no human cells remained. The cell retention further led to bone formation by the human cells. In contrast, the constructs of shorter pre-culture period (T-6) allowed for an influx of rat osteoprogenitor cells that participated 
in the bone formation, resulting in bone tissue composed of a mixture of human and rat-derived collagen type I. This is in concert with previous findings, in which osteogenic differentiation indeed improved when human cells were retained in implanted constructs [299], thereby participating in bone formation [273, 300].

Osteoblasts develop strong adhesion sites with substrates and a tight ECM, which shields the construct from cellular infiltration [267]. Osteoprogenitor cells on the other hand, are highly proliferative and can easily migrate on substrates due to weak adhesion, combined with increased cell traction and detachment [267]. This, together with deposition of immature ECM, makes the immature construct prone to cellular influx, including osteoprogenitor cells, capillaries and (foreign body) MGCs, with subsequent construct remodeling and clearance of the implanted human cells (Supplementary Figure 5.4). This indicates that altered duration of the pre-differentiation period of the bioengineered construct can tune the origin and onset of the bone formation after implantation.

Prolonging the in vitro pre-culture period of our MSC/ECFC Co-culture model was previously shown to produce a more mature vascular network over time [206, 294]. Having a mature vascular network before implantation was hypothesized to favor a fast connection to the host circulation, as described elsewhere [301, 302]. Interestingly, when prolonging the pre-differentiation time, we observed a degradation of the vascular network in vitro in the vicinity of the CaP granules (but not in vivo). This is in stark contrast to the previously reported positive effect of CaPs on vasculogenesis in [84, 281-284] (and reviewed in [279]). This adverse effect was most likely caused by a specific surface reactivity between the type of BCP granules and the hydrogel in our system, with limited ionic exchange kinetics through the hydrogel in this static in vitro set-up. It has previously been reported that high levels of inorganic phosphates and calcium can induce apoptosis in endothelial cells, as seen in patients with hyperphosphatemia [303, 304]. However, this complex ionic reactivity of $\mathrm{CaP}$ with the hydrogel is out of the scope of the present study and is the focus of ongoing work.

Despite of this, all constructs were well vascularized at the time of explantation and capillaries were identified throughout the whole construct. This implies that at that time a sustained supply of oxygen and nutrients was available and that the constructs' vascularization was not impaired by the presence of the CaP granules as seen in vitro. However, no human endothelial cells could be retrieved in the implanted constructs and all vessels were derived from the ingrowth of $\mathrm{CD}_{3} 4^{+}$rat endothelium. No differences in the vessel volume were found among groups, indicating that at the time of explantation there was no effect of the construct's prevascular state, ranging from naive cells (T-1), prevascularization (T-6) or vascular remnants (T-14). Notably, not all small vessels and capillaries were perfused with the radiopaque agent, which could cause an underestimation of the 
vessel volume. However, the IHC-based quantification also showed that there were no differences in the level of vascularization among the groups.

Whilst generally an additional effect of a pre-vascular network on osteogenesis and/or anastomosis is reported after implantation of CaP-containing constructs [272, 283, 285, $287,290,292,305]$, this effect was absent in our study. Nevertheless, the cited studies mostly focused on the added effect of endothelial cells on top of osteoprogenitors, which was not the focus of our study. Also, it should be noted that comparing the T-1, T- 6 and $\mathrm{T}-14$ groups to each other is a complex matter, since there are at least two variables that differ per time-point: the level of vasculature and the osteogenic differentiation. This makes a one-on-one comparison of the pre-vascularized (T-6) versus non-vascularized conditions (T-1, $\mathrm{T}-14)$ challenging.

Yet, it remains unclear why no human ECs could be traced back in any of the constructs, including the T-6 group, which upon implantation still supported a considerable vascular network. It may be that the effect of the vascular network was mostly visible in the first weeks of implantation, and was diminished at the end point ( 8 weeks). It has been reported that anastomosis of host to donor vasculature indeed occurs in the first week after implantation [301, 302, 306], and the additive effect of pre-vascularization was shown to be mainly important during this early time period and could be stabilized after this initial phase [307]. Furthermore, it may be that anastomosis occurred too late for the pre-established vascular network of the T- 6 group to survive. In general, only a mature vascular network promotes anastomosis with the host vasculature [301]. Nevertheless, no necrotic core was detected in any of the samples and all constructs were well vascularized after 8 weeks, possibly promoted by the remaining mural-like cells in the constructs [301].

Overall, we have used a model, in which the in vitro behavior of osteogenic cells and vasculogenesis in combination with biomaterials can be closely monitored. The model consists of a hybrid hydrogel that efficiently supports vasculogenesis and osteogenesis in vitro and outperforms the golden standard Matrigel in this matter [294]; it contains clinically relevant progenitor cell types and follows the natural pattern of vasculogenesis based on tight cross-communication with the osteogenic cells. Having the ability to follow the fluorescently tagged ECs freely also gives a better indication of the reaction of ECs towards biomaterials e.g. CaP bioceramics and their localized effects. For the first time, it became clear that the addition of CaPs can have a negative effect on the vasculature in an in vitro hydrogel co-culture. This phenomenon that has not been presented previously emphasizes the need for complex multi-tissue in vitro models and detailed observations on the cellular behavior towards biomaterials. This is especially important when aiming to translate or link in vitro models to an in vivo situation. Nevertheless, host vasculature did form in the vicinity of CaP granules in vivo, which is in line with previous studies [272, 
$278,283,285-293]$. Additionally, the CaPs enabled in vivo bone formation of the co-culture model in the hydrogel, which would otherwise not occur.

Most importantly, we revealed that by altering the length of the pre-differentiation period we can specifically tune the origin of the bone tissue. This opens up the discussion of what the most favorable situation would be: an early onset of bone formation by the donor cells, or host cell participation in the bone forming process. The implanted constructs indeed evoked participation of migrated recipient cells in the osteogenic process, indicating that even in the case of full donor cell clearance, bone formation did proceed. This matter may be desirable if striving for the regeneration of patient-derived bone. Future directions should also be guided towards an improved understanding of the adverse effects of the $\mathrm{CaP}$ granules on vasculature in vitro and if and how these need to be resolved to further improve the in vivo outcomes.

\section{Acknowledgements}

This research was partially funded by an NWO (Netherlands Organization for Scientific Research) Graduate Program Grant (022.005.018). We thank Kuros Biosciences for providing us with the bioceramic materials. Moreover, we thank Florence de Groot and Jacqueline Alblas for the discussions and input, professor Harrie Weinans for the $\mu C T$ input and Mattie van Rijen, Leanne de Silva and Barbara Klotz for their technical support.

\section{Supplementary information}

\subsection{Supplementary Materials and Methods}

\subsubsection{Endothelial colony forming cells (ECFCs)}

In short, ECFCs were isolated from human cord blood. The procedure was approved by the medical research ethics committee, University Medical Center Utrecht; informed consent was obtained from the mother. Confirmation of the ECFCs' potency was performed by phenotypic characterization with flow cytometry as presented previously [206]. After isolation, the ECFCs were either frozen or further expanded in complete endothelial growth medium-2 (EGM-2) containing Endothelial Basal Medium-2 + SingleQuots (Lonza), $100 \mathrm{U} /$ $\mathrm{mL}$ penicillin and $100 \mu \mathrm{g} / \mathrm{mL}$ streptomycin (PenStrep, Gibco), and 10\% heat-inactivated Fetal Bovine Serum (FBS) (S14068S1810, Biowest)

\subsubsection{Multipotent Mesenchymal stromal cells (MSCs)}

MSCs were isolated and characterized as previously described [206]. In short, MSCs from human bone marrow aspirates were obtained from consenting patients $(n=2$.) The procedure was approved by the local medical research ethics committee, University Medical Center Utrecht. These isolates are referred to as MSC1 (female, age 20) and MSC2 (female, age 60). The MSCs were phenotypically characterized by their cell surface 
marker expression profiles with flow cytometry. Their multipotency was confirmed via differentiation towards adipogenic, osteogenic, and chondrogenic lineages. After isolation, MSCs were further expanded in MSC expansion medium, consisting of aMinimum Essential Medium (Gibco Paisley, 22561), supplemented with 10\% FBS, 100 U/ $\mathrm{mL}$ penicillin and $100 \mu \mathrm{g} / \mathrm{mL}$ streptomycin (PenStrep, Gibco), $0.2 \mathrm{mM} \mathrm{L-ascorbic} \mathrm{acid-2-}$ phosphate (ASAP, Sigma), and $1 \mathrm{ng} / \mathrm{mL}$ basic fibroblast growth factor (rh-FGF-2; R\&D Systems). The MSCs were encapsulated in the construct at passage 4 (in vitro analysis) and passage 3 (in vivo implantation).

\subsubsection{Tissue mineralization - $\mu C T$ scanning}

$\mu C T$ scans were performed to assess tissue mineralization at 2, 4, 6 and 8 weeks under anesthesia. A baseline $\mu \mathrm{CT}$ scan was made directly after implantation of the construct (scan time: $3 \mathrm{~min}$; voxel size: $42 \mu \mathrm{m}^{3}$; X-ray energy and X-ray tube potential: $90 \mathrm{keV}$; X-ray intensity: $180 \mu \mathrm{A}$; Quantum FX, PerkinElmer). Images were converted to 8-bit images, ROIs were segmented with a global threshold and inverted. Bone volume was reconstructed and measured in $\mathrm{mm}^{3}$ with the ImageJ plugin BoneJ [296] and the ROI manager function. Three-dimensional (3D) reconstructions of the defects were made using image processing software (Fiji). For the analysis of the $\mu \mathrm{CT}$ results, the data was checked for a normal distribution using the shapiro-wilk test and the absence of outliers was confirmed using the ROUT test $(\mathrm{Q}, 1 \%)$. Hereafter, a two-way ANOVA repeated measurements was used to test for differences between the group, and the tests were adjusted for multiple comparisons by a Tukey post hoc comparison test.

\subsubsection{Osteoclasts}

To present osteoclast activity, a Tartrate-resistant acid phosphatase (TRAP) staining was performed on the slides. Slides were incubated with $0.2 \mathrm{M}$ acetate buffer - tartaric acid ( $\mathrm{pH} \mathrm{5.0)} \mathrm{for} 20 \mathrm{~min}$ at room temperature. Then $0.5 \mathrm{mg} / \mathrm{ml} \mathrm{Naphthol} \mathrm{AS-MX} \mathrm{phosphate} \mathrm{and}$ $1.1 \mathrm{mg} / \mathrm{mL}$ fast red TR salt were added and incubation was pursued until the osteoclasts were bright red (1-4 hours). Samples were counterstained with Mayer's hematoxylin and mounted in ecomount (Biomedical care).

\subsubsection{Gene expression}

Surplus samples that were not implanted were used for in vitro gene expression analysis ( $n=6$ per group). Samples for total cellular RNA extraction were washed in PBS and resuspended in $0.5 \mathrm{ml}$ Trizol Reagent (Thermo Fischer Scientific) for $5 \mathrm{~min}$ at room temperature and stored at $-80^{\circ} \mathrm{C}$ till further use. After thawing, samples were spun down at $12000 \mathrm{G}$ for $10 \mathrm{~min}$ to separate the granules from the cellular extraction. Hereafter, the supernatant was mixed with $20 \%$ Chloroform (Millipore) and centrifuged at $12000 \mathrm{G}$ for $15 \mathrm{~min}\left(4^{\circ} \mathrm{C}\right)$. The upper aqueous phase was then mixed with 2-propanol (Sigma) and 0.5 $\mu \mathrm{l}$ glycogen (R0551, Thermo Fisher Scientific), and left to incubate at room temperature 
for $10 \mathrm{~min}$. Samples were again centrifuged at $12000 \times \mathrm{g}$ for $10 \mathrm{~min}\left(4^{\circ} \mathrm{C}\right)$, the pellet was washed in $75 \%$ ethanol and spun down at $7500 \times \mathrm{g}$ for $5 \mathrm{~min}\left(4^{\circ} \mathrm{C}\right)$. After drying, the pellet was re-suspended in $10 \mu \mathrm{l}$ of RNase-free water and exposed to DNAse treatment (AM2238, Ambion).

Hereafter, the total RNA ( $5 \mathrm{ng} / \mu \mathrm{l}$ input for all samples) was reverse-transcribed into cDNA with an iScript CDNA synthesis kit (170-8890, Biorad). The quantitative PCR (qPCR) reaction mix was prepared with FastStart SYBR green master (04673484001, Sigma) with an end volume of $10 \mu \mathrm{l}$ containing $10 \mathrm{ng}$ of cDNA and $500 \mathrm{nM}$ primer (forward and reversed). Glyceraldehyde-3-phosphate dehydrogenase (GAPDH) was selected as housekeeping gene among a set of candidates, based on its expression stability tested with the algorithm from Normfinder. Primer sequences and annealing temperatures can be found in Supplementary Table 2. Primers were optimized for their amplification efficiency (calculated via: $E=-1+10^{-1 / \text { slope }}$ ).

\subsection{Supplementary Results}

\section{$\mu C T$ analysis}

All groups showed a slight increase in signal in the first 4 weeks, with a mild decrease in signal from 4-6 weeks (T-1 and T-14 groups) and subsequent increase till week 8 again. The T-6 samples showed a steady state over time (Supplementary Figure $5.2 \mathrm{H}$ ). After 4 weeks of implantation, the signal of the T-1 group was tested significantly higher compared to the T- $6(p=0.047)$ and T-14 $(p=0.041)$. Three samples of the T-1 group showed areas with mineralized tissue on top of the construct in the $\mu \mathrm{CT}$ images, but these areas could not be linked to areas of bone formation in the paraffin/MMA slides. However, no clear differences in extent of mineralization over time could be found when looking at the measurements of the individual samples or when grouped in samples with bone formation versus no bone formation (Supplementary Figure $5.2 \mathrm{H}-\mathrm{J}$ ). Due to the high radiopacity of the ceramic material, we believe that the newly formed bone was overshadowed by scattering and the relatively large working field of view prevented the detailed analysis of the data. Also, the most abundant tissue in the construct was osteoid, which does not contain minerals yet. This can therefore lead to an underestimation of the bone forming capacity of the samples. Therefore, it was decided that the quantification of bone formation will solely be based on histochemical staining (paraffin + MMA) (Figure 5.4). 


\subsection{Supplementary Figures}

Supplementary Table 1. Specifics of the antigen retrieval method, primary and secondary antibodies used for the IHC/fluorescent analyses.

\begin{tabular}{|c|c|c|c|c|}
\hline $\begin{array}{l}\text { Primary } \\
\text { antibodies }\end{array}$ & Target antigen & Concentration & Antigen retrieval & Secondary antibody \\
\hline \multirow{2}{*}{$\begin{array}{c}\text { Pericytes } \\
\text { (fluorescent- } \\
\text { whole mount) }\end{array}$} & aSMA, Clone 1A4, Cy3 & & & \\
\hline & Sigma Aldrich & $0.5 \mathrm{ug} / \mathrm{ml}$ & - & - \\
\hline $\begin{array}{l}\text { Osteoblasts } \\
\text { (IHC - paraffin) }\end{array}$ & $\begin{array}{l}\text { Osteonectin } \\
\text { DSHB Hybridoma } \\
\text { Product AON-1 }\end{array}$ & $\sim 9 \mathrm{ug} / \mathrm{ml}$ & $\begin{array}{c}\text { citrate buffer } \\
\left(10 \mathrm{mM}, \mathrm{pH} 6,80^{\circ} \mathrm{C}\right) \\
\text { for } 20 \mathrm{~min}\end{array}$ & $\begin{array}{l}\text { Brightvision poly } \\
\text { HRP-Anti-Rabbit lgG, } \\
\text { Immunologic (30 min) }\end{array}$ \\
\hline \multirow{3}{*}{$\begin{array}{l}\text { Matrix origin } \\
\text { (IHC - paraffin) }\end{array}$} & $\begin{array}{l}\text { mouse anti-human Anti- } \\
\text { mitochondria } \\
113-1, \text { AB92824, Abcam }\end{array}$ & $1 \mu \mathrm{g} / \mathrm{mL}$ & $\begin{array}{c}\text { TRIS-EDTA }(10 \mathrm{mM} \\
\text { TRIS- } \mathrm{HCl} / 1 \mathrm{mM} \\
\text { EDTA, pH 9, } 30 \\
\text { minutes } 90^{\circ} \mathrm{C} \\
\end{array}$ & $\begin{array}{l}\text { Brightvision poly } \\
\text { HRP-Anti-mouse lgG, } \\
\text { Immunologic ( } 30 \text { min) }\end{array}$ \\
\hline & $\begin{array}{l}\text { rabbit anti-human } \\
\text { collagen type } 1 \\
\text { EPR7785, ab138492, } \\
\text { Abcam }\end{array}$ & $2.2 \mu \mathrm{g} / \mathrm{nL}$ & $\begin{array}{c}\text { pronase } 1 \mathrm{mg} / \mathrm{mL} \\
\text { and hyaluronidase } \\
10 \mathrm{mg} / \mathrm{mL}, 20 \\
\text { minutes }\end{array}$ & $\begin{array}{c}\text { Brightvision poly } \\
\text { HRP-Anti-Rabbit lgG, } \\
\text { Immunologic (30 min) }\end{array}$ \\
\hline & $\begin{array}{l}\text { Rabbit anti-Rat collagen } \\
\text { type } 1 \\
\text { abt123, Chemicon/ } \\
\text { Millipore }\end{array}$ & $20 \mu \mathrm{g} / \mathrm{mL}$ & $\begin{array}{c}\text { citrate buffer }(15 \\
\text { minutes, } 90^{\circ} \mathrm{C}\end{array}$ & $\begin{array}{c}\text { Brightvision poly } \\
\text { HRP-Anti-Rabbit lgG, } \\
\text { Immunologic (30 min) }\end{array}$ \\
\hline \multirow{3}{*}{$\begin{array}{c}\text { ECs } \\
\text { (IHC- paraffin) }\end{array}$} & $\begin{array}{l}\text { Goat anti-rat CD34 } \\
\text { AF4117; R\&D Systems }\end{array}$ & $0.01 \mathrm{mg} / \mathrm{mL}$ & $\begin{array}{l}30 \text { min incubation } \\
\text { at } 37^{\circ} \mathrm{C} \text { with } 0.1 \% \\
\text { trypsin }\end{array}$ & anti-goat HRP (1 hour) \\
\hline & $\begin{array}{l}\text { mouse anti-human } \\
\text { CD31 M0823, Dako }\end{array}$ & $0.13 \mathrm{mg} / \mathrm{mL}$ & $\begin{array}{c}10 \mathrm{mM} \text { citrate } \\
\text { buffer, } \mathrm{pH} 6,90^{\circ} \mathrm{C}\end{array}$ & $\begin{array}{l}\text { Brightvision poly } \\
\text { HRP-Anti-mouse lgG, } \\
\text { Immunologic (30 min) }\end{array}$ \\
\hline & $\begin{array}{l}\text { rabbit anti-GFP } \\
\text { 6AT316, ab38686, } \\
\text { Abcam }\end{array}$ & $0.0039 \mathrm{mg} / \mathrm{mL}$ & $\begin{array}{c}10 \mathrm{mM} \text { citrate } \\
\text { buffer, } \mathrm{pH} 6,90^{\circ} \mathrm{C}\end{array}$ & $\begin{array}{c}\text { Brightvision poly } \\
\text { HRP-Anti-Rabbit lgG, } \\
\text { Immunologic (30 min) }\end{array}$ \\
\hline
\end{tabular}


Supplementary Table 2. Primers used in $q P C R$ with annealing temperature of $60^{\circ} \mathrm{C}$.

\begin{tabular}{lll}
\hline Protein/Gene & Forward sequence & Reverse sequence \\
\hline GAPDH & ATGGGGAAGGTGAAGGTCG & TAAAAGCAGCCCTGGTGACC
\end{tabular}

\begin{tabular}{lll}
\hline aSMA/ACTA1 ATGCCATCATGCGTCTGGAT & ACGCTCAGCAGTAGTAACGA
\end{tabular}

CD31/PECAM1 GCAGTGGTTATCATCGGAGTG TCGTTGTTGGAGTTCAGAAGTG

CLAP/NG2 GAAGGAGGACGGACCTCAAG GATCAGCTGCTCTTCCACCATT

\begin{tabular}{lll}
\hline RUNX2 & TTACAGTAGATGGACCTCGGGA & AGGAATGCGCCCTAAATCACT
\end{tabular}

\begin{tabular}{lll}
\hline SPP1/OPN GCCGAGGTGATAGTGTGTT & GTGGGTTTCAGCACTCTGGT
\end{tabular}

BGLAP/OCN CCTCACACTCCTCGCCCTAT GCTTGGACACAAAGGCTGCAC

COL1A1/Col I AAGAGGAAGGCCAAGTCGAG GTTTCCACACGTCTCGGTCA




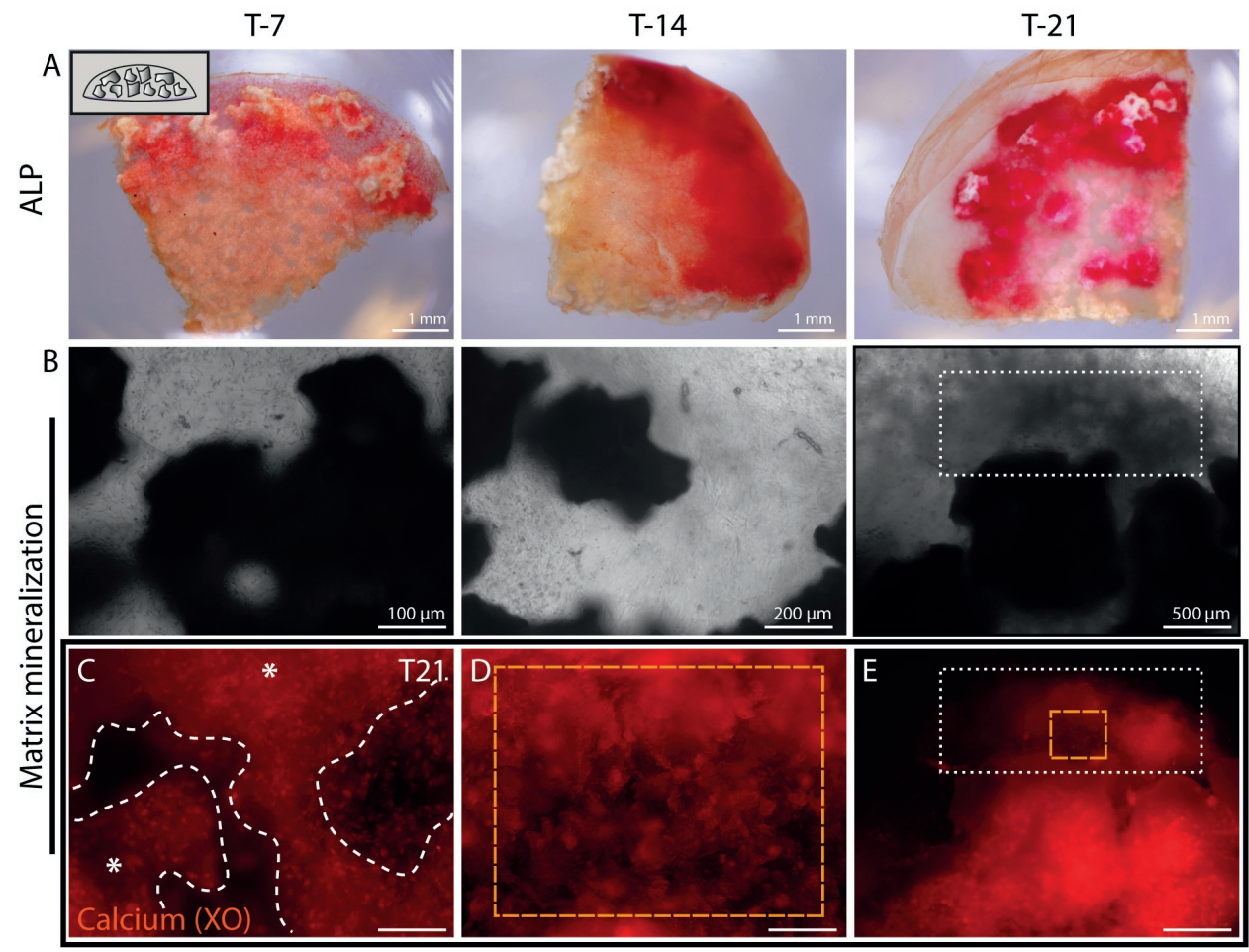

Supplementary Figure 5.1. In vitro osteogenic differentiation of cell-laden small CaP gelatin-PEG hydrogels. A) staining for ALP activity (red) in small hydrogels. The activity increased till day 14 and was confined to the vicinity of the granules at day 21. B) Between day 14 and day 21 the matrix became dark of appearance in the bright field images (dotted white box corresponds to box in E). C) Cellular calcium deposition ( $\mathrm{XO}$, red) was also increased after 21 days and the $\mathrm{D}$ ) dark matrix stained positive for $\mathrm{XO}$ (orange box is a magnification of the orange box in E). E) Fluorescent image of the $\mathrm{XO}$ signal from the same location as in image $\mathrm{B}$. The white dotted box shows that the dark matrix of B) is positive for XO. 

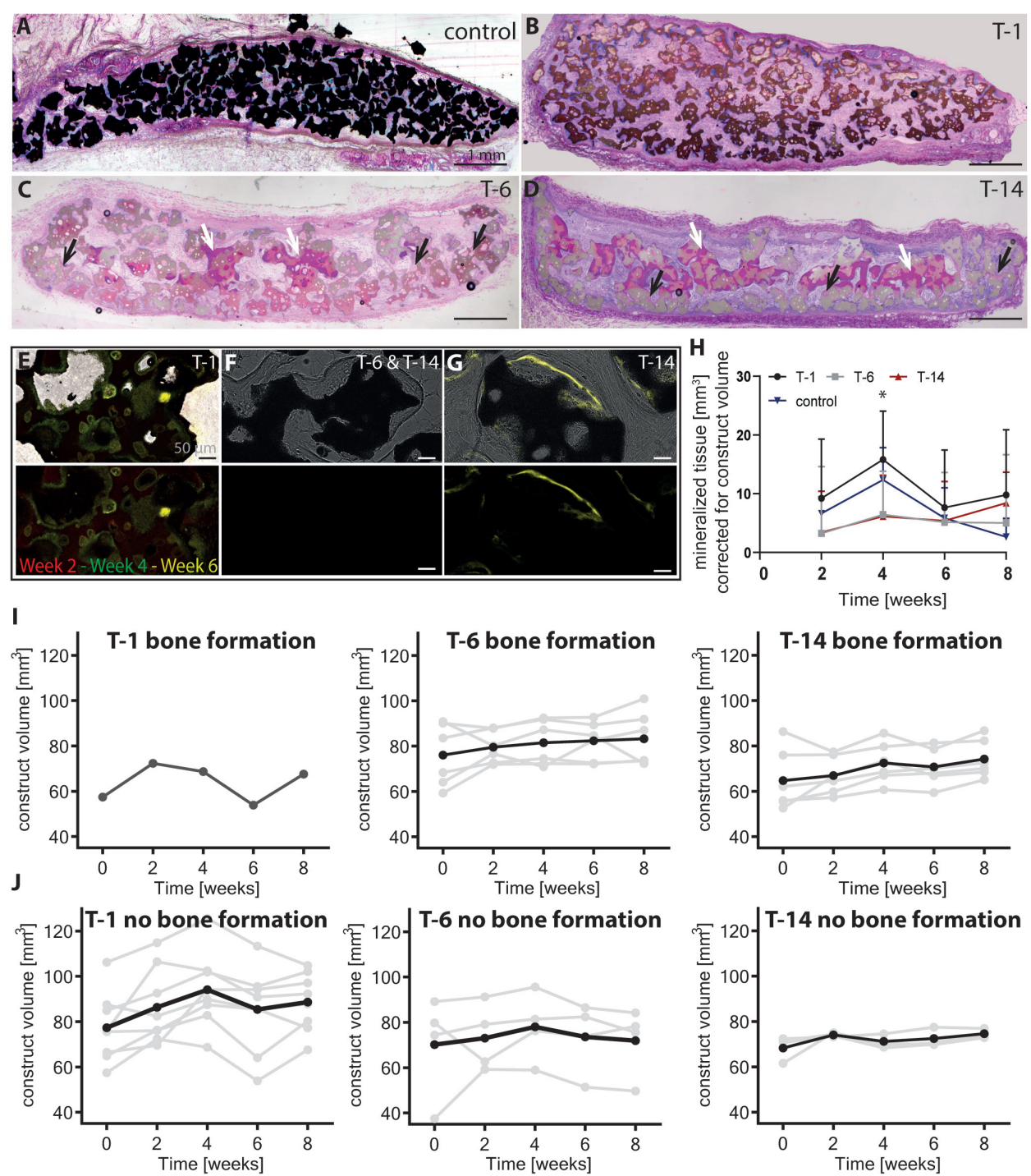

Supplementary Figure 5.2. Construct mineralization over time after implantation. Cross-sectional overviews of MMA slides of the control A), T-1 B), T-6 C) and T-14 group D). E) MMA samples from the T-1 group showed no bone formation, but some positive fluorochrome signal at the granules. F) Both the samples from the T- 6 and T-14 groups show bony tissue around the granules without a positive signal for any of the fluorochromes. G) Locations with only yellow signal (mineralization after 6 weeks) were frequently observed in the samples from the T-14 groups. H) $\mu C T$ analysis of the scans performed over the time, corrected for the construct volume at day 0 . The samples of T-1 had a higher signal compared to T-6 $(p=0.047)$ and T-14 $(p=0.041)$ after 4 weeks of implantation. $\mathrm{I}-J$ ) Absolute $\mu \mathrm{CT}$ of the individual samples (each line represents one sample); I) for samples with bone formation and J) samples without bone formation. The black line represents the mean of the samples, gray lines indicate the individual samples. 


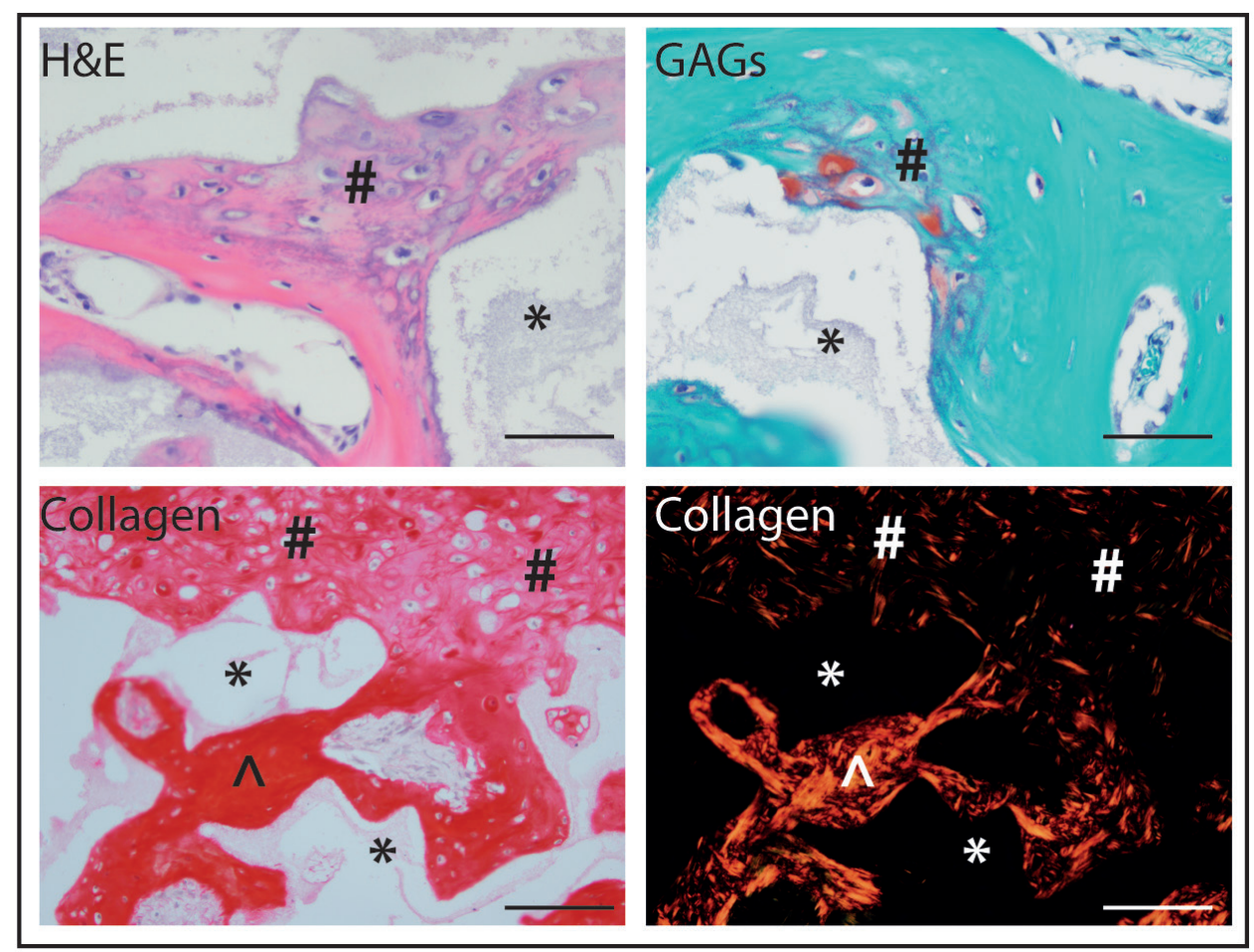

Supplementary Figure 5.3. Mineralized cartilage formed during implantation. Some bone forming samples (independent of group/pre-culture time) contained mineralized cartilage (\#), positive for glycosaminoglycans (GAGs, safranin-O staining, red) and showed a disorganized and more loose collagen organization (Picrosirius red), compared to bone $(\wedge)$. Samples were visualized with bright field imaging or polarized light. Scale bars represent $50 \mu \mathrm{m}$. 


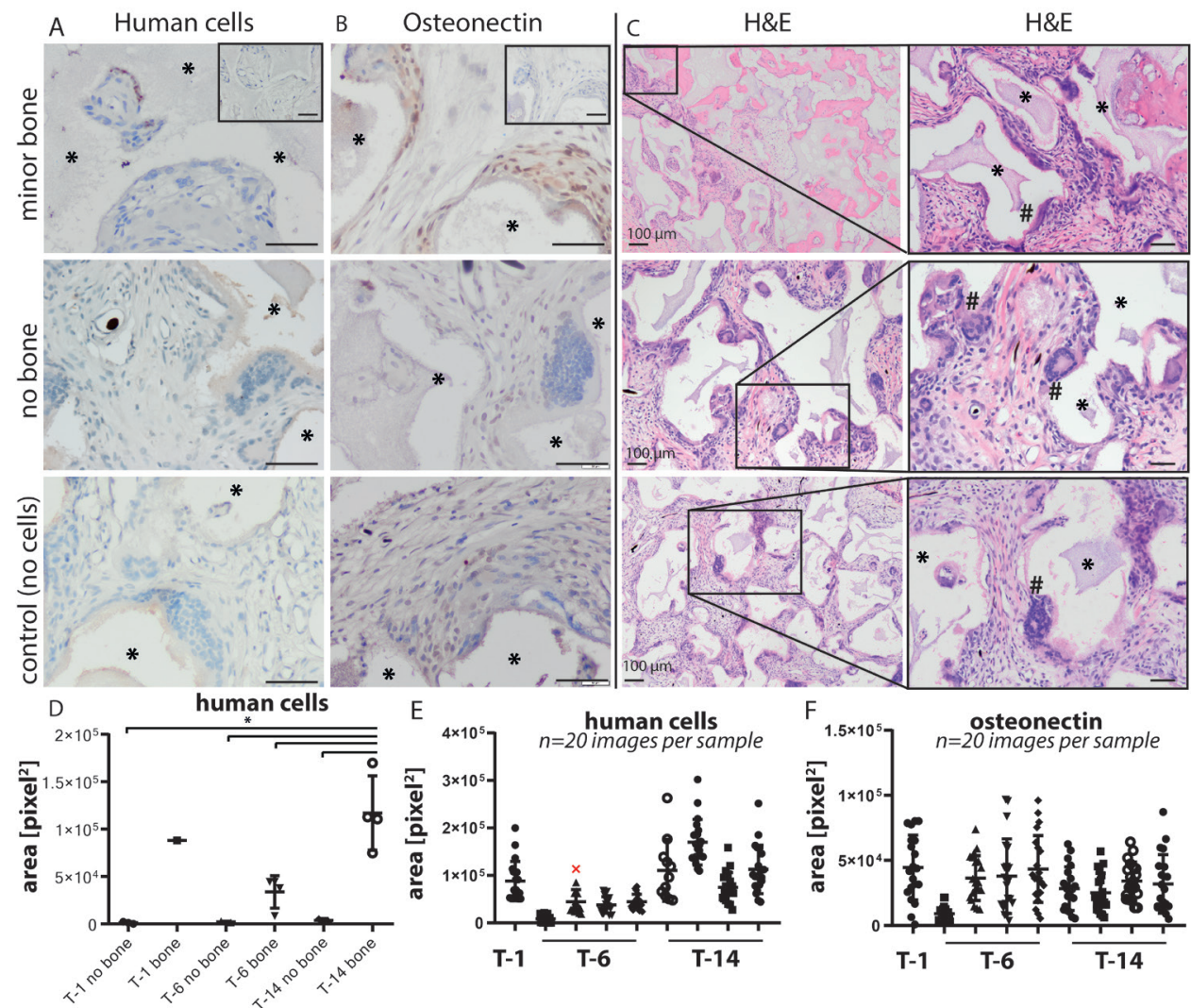

Supplementary Figure 5.4. Differences in samples with or without bone formation. Paraffin slides of samples with minor bone formation compared to samples without bone formation or to the control situation. Slides were stained for A) human cells, B) osteonectin or C) evaluated with an hematoxylin staining. Inset represent the isotype control. C) The H\&E staining shows the overall morphology of the construct. Multi-nucleated giant cells (MGCs, \#) were only observed in areas without bone. Samples without bone formation were completely infiltrated with MGCs, and located at the edge of the granules $\left(^{*}\right)$. E) Quantification of the human cells in the non-bone forming samples confirmed that there were no human cells left when no bone was formed $\left.{ }^{*} p<0.006\right)$. Graph represent avaraged values of 20 images/slide, for 1 slide per sample. E-F) For the quantification of the human cells and osteonectin positive cells, 20 images were taken throughout the sample and the pixel ${ }^{2}$ was averaged. Outliers are identified with ' $x$ '. Scale bars represent $50 \mu \mathrm{m}$ unless indicated otherwise. 

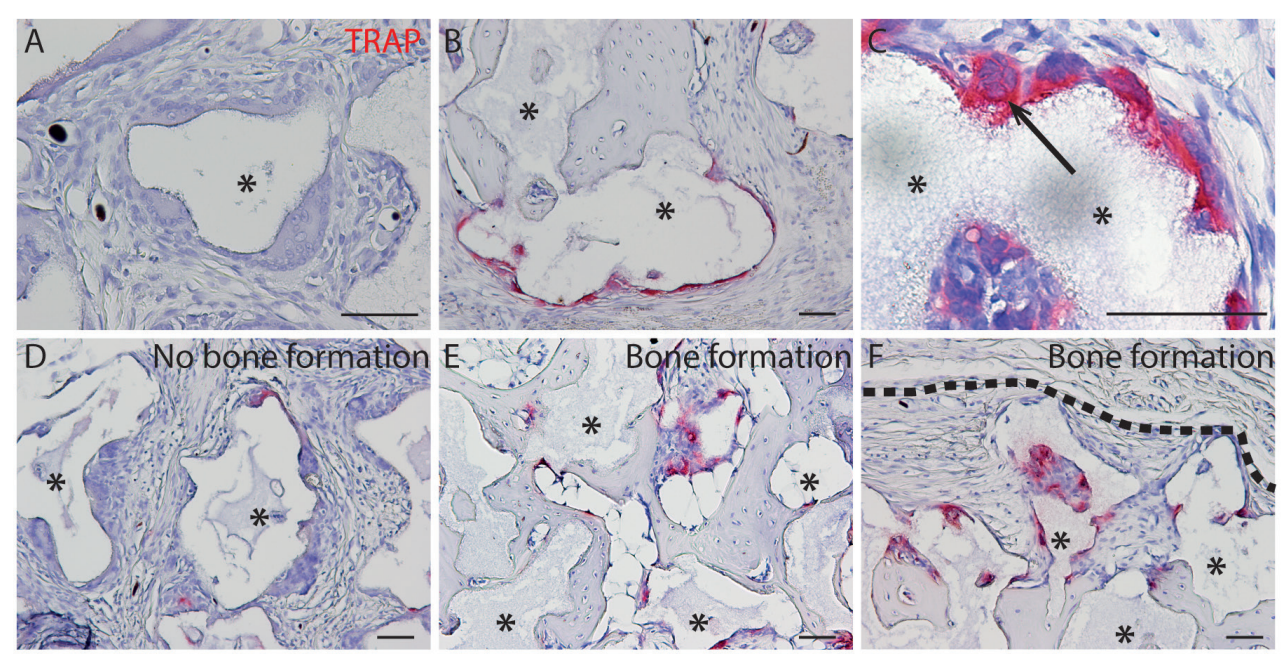

Supplementary Figure 5.5. Tartrate-resistant acid phosphatase (TRAP) staining. A) MGCs were located around the granules $\left(^{*}\right)$. B) Sample with bone formation showed cells positive for TRAP at the edge of the granule but were not located at the edge of the bone. C) TRAP-positive cells were multi-nucleated (black arrow) and found in both, samples D) without and E) with bone formation. F) In samples with bone formation, TRAP cells were mostly found at the edge of the construct (dotted black line). Scale bars represent $50 \mu \mathrm{m}$. 





Osteogenic stimulation interferes with the maintenance of capillary-like networks in osteogenic constructs

Manuscript in preparation:

I. Pennings, C. Cheng, A.J.W.P Rosenberg, D. Gawlitta

Preliminary results 


\section{Abstract}

Bioengineered constructs with calcium phosphate (CaP) ceramics can be used for the reconstruction of critical-size bone defects. Inclusion of a perfusable vascular network would maintain the cellular viability and would facilitate upscaling towards viable clinically relevant sized bone implants. Pre-vascularization of $\mathrm{CaP}$-containing osteogenic constructs can be achieved by co-culturing multipotent mesenchymal stromal cells (MSCs) with endothelial colony forming cells (ECFCs) in a gelatin-PEG hydrogel. These constructs were previously shown to facilitate bone formation after in vivo implantation, but required to undergo osteogenic pre-differentiation before implantation to achieve this. However, during the in vitro pre-differentiation period, we observed that CaPs had a negative effect on the maintenance of the prevascular network. This study was set-up to narrow down the specific factor(s) that caused the adverse effects of the presence of $\mathrm{CaP}$ in our co-culture model on in vitro vasculogenesis.

Human MSCs and ECFCs were co-cultured with biphasic 35/65\% hydroxyapatite $(\mathrm{HA}) / \beta$-tri-calcium-phosphate ( $\beta$-TCP) granules to pinpoint the origin of the vascular degradation: i) topography of the bioceramics, ii) combination of $\mathrm{CaP}$ granules with the type of hydrogel, iii) type of culture medium, and iv) dissolution products of the bioceramics $\left(\mathrm{Ca}^{2+}\right.$ and $\left.\mathrm{PO}_{4}^{-}\right)$. Outcome parameters were based on the vascular development and maintenance (total network length) and osteogenic differentiation (alkaline phosphatase (ALP) and mineralization).

The co-culture model allowed for the formation of a capillary-like network that was supported by mural cells. However, the network regressed in the vicinity of the CaP ceramics, independent of i) surface topography, ii) hydrogel system, iii) and type of culture medium. The preliminary data presented here, shows that the vascular regression was related to an osteogenic overstimulation with high levels of inorganic phosphate (Pi). Additionally, a second event involving vascular regression was observed, but now in the gelPEG co-cultures in which there were no CaP granules added. Here, calcification of capillaries was found to induce a regression of the pre-vascular network, also coinciding with high levels of $\mathrm{Pi}$. This indicates that tipping the balance too much towards an osteogenic environment can result in an unfavorable environment for vasculogenesis. Solutions are proposed to prevent adverse the adverse effect (e.g., phosphate channel blockers). 


\section{Introduction}

Reconstructions of critical-size (mandibular) bone defects are often carried out by transplantation of autologous bone grafts to the defect site, from which the clinical applicability may be hampered by restricted availability and donor-site morbidity. These drawbacks can be circumvented by developing patient-specific bone constructs via regenerative medicine. As such, engineering of bone analogues can be realized by the use of synthetic bone graft substitutes or bioengineered constructs. Among the synthetic bone graft substitutes, calcium phosphate (CaP) bioceramics have a high potential for the realization of adequate bone grafts, owing to their resemblance of the native bone minerals, resorption properties and potential to promote bone formation $[266,267]$.

The addition of differentiated or non-differentiated osteoprogenitor cells to CaP constructs has been shown to enhance regeneration, and moreover is vital for bone formation in small animal models [269-274, 292, 297]. CaPs have therefore already been combined with numerous combinations of osteoprogenitor cells, growth factors and hydrogels [24, 308]. However, an intrinsic functional vascular network, and its connection to the surrounding vascular network upon implantation, is essential to maintain the viability of osteoprogenitor cells in large (cm-scale) constructs. One of the approaches for the vascularization of CaP-based cell-seeded constructs is the addition of endothelial cells to the construct, which have the ability to self-assemble into a vascular network [84, 281-284].

Previously, a pre-vascularization co-culture model consisting of multipotent mesenchymal stem cells (MSCs) and endothelial colony forming cells (ECFCs) in a gelatin-PEG-based hydrogel system was combined with 35/65\% hydroxyapatite (HA)/ $\beta$-tri-calciumphosphate ( $\beta$-TCP) granules (chapter 5 ). These constructs showed high rates of bone formation after in vivo implantation in an ectopic location in immunocompromised rats, but only when they were osteogenically pre-differentiated. However, the CaPs seemed to have a negative effect on the pre-vascularization in vitro. It was observed that the network regressed in the vicinity of the granules when the pre-culture period was extended beyond 7 days. This adverse effect was not observed in the in vivo situation, as ingrowth of rat-derived capillaries was not inhibited in the presence of granules and were located in close vicinity of the ceramics.

Since an in vitro pre-culture time was required for the in vivo bone formation, we sought to find the factor of the CaPs that influenced this vascular regression. This would enable us to osteogenically pre-differentiate in vitro while maintaining the capillary-like network. We hypothesized that the conflicting effect was most likely caused by a specific surface reactivity between the type of $\mathrm{CaP}$ granules and the hydrogel in our static culture set-up. In this chapter, we provide preliminary data to substantiate this hypothesis. 


\section{Materials and Methods}

\subsection{Study design}

This study was set up to determine the specific factor(s) that cause(d) the previously reported (chapter 5) adverse effects of $\mathrm{CaP}$ granules on in vitro vasculogenesis. Hydrogel co-culture droplets ( $20 \mu \mathrm{L}$ or $75 \mu \mathrm{L}$ ) were made by combining human MSCs and ECFCs in a hydrogel with or without CaP granules (Figure 6.1 A). Constructs were cultured for up to 10 days to evaluate their osteogenic and vasculogenic differentiation. The contribution of four possible causes to the vascular degradation were assessed: 1) surface topography of the CaPs, 2) CaP combination with the hydrogel, 3) type of culture medium, and 4) dissolution products of the CaP granules. To evaluate the first topic, two types of biphasic $35 / 65 \%$ HA/ $\beta$ TCP ceramics were added with different surface topographies (submicron versus micron surface topography). The influence of the hydrogel system or the type of medium was examined by culturing in gelatin-PEG [294] or Matrigel [206]; or in endothelial growth medium, MSC expansion medium or osteogenic differentiation medium, respectively. The fourth potential cause was investigated by addition of the individual or combined dissolution products $\left(\mathrm{Ca}^{2+}\right.$ and $\left.\mathrm{PO}_{4}^{-}(\mathrm{Pi})\right)$ in the medium or in the hydrogel, or by adding the CaP granules to the medium. Outcome parameters were based on the vascular development (total network length, apoptosis), and osteogenic differentiation (qualitative presence of alkaline phosphatase (ALP) or mineralization (xylenol orange (XO)).

\subsection{Cell culture and characterization}

\subsubsection{Cell culture}

ECFCs and MSCs were isolated and characterized as described previously [206]. The procedure was approved by the medical research ethics committee, University Medical Center Utrecht; informed consent was obtained. ECFCs were subcultured in complete endothelial growth medium-2 (EGM-2) containing Endothelial Basal Medium-2 + SingleQuots (Lonza), $100 \mathrm{U} / \mathrm{mL}$ penicillin and $100 \mu \mathrm{g} / \mathrm{mL}$ streptomycin (PenStrep, Gibco), and 10\% heat-inactivated Fetal Bovine Serum (FBS-HI; Biowest S14068S1810). Fluorescently tagged ECFCs were generated in passage 5 with a lentiviral green fluorescent protein (GFP) construct or red fluorescent protein from discosoma striata (dsRed) construct (in a pHAGE2 vector combined with a human EF-1a promotor) in FBS-free medium, as described previously [103]. The cells were used for construct encapsulation at passages 11-13. MSCs were isolated and expanded in MSC expansion medium, consisting of a-Minimum Essential Medium (Gibco Paisley, 22561) with 10\% FBS, $100 \mathrm{U} / \mathrm{mL}-100$ $\mu \mathrm{g} / \mathrm{mL}$ PenStrep, $0.2 \mathrm{mM}$ L-ascorbic acid-2-phosphate (ASAP, Sigma), and $1 \mathrm{ng} / \mathrm{mL}$ basic fibroblast growth factor (bFGF) (rh-FGF-2; R\&D Systems). The MSCs were encapsulated in the constructs at passage 3 or 4 . 


\subsection{Hydrogel cultures}

\subsubsection{Gelatin-PEG (gelPEG) hydrogel preparation}

Hydrogels were fabricated as previously described [294, and chapter 5). In brief, PEG-GIn was synthesized by conjugating an eight-arm PEG-vinyl sulfone (8-PEG-VS, mol wt 40 $\mathrm{kDa}$ ) to a Gln-sequence (H-NQEQVSPL-ERCG-NH 2 $^{\prime}$ TG-GIn). The modified PEG (PEG-GIn) was then covalently crosslinked with Factor XIII (Cluvot $250 \mathrm{IE}$, CSL Behring) to the native lysine residues on gelatin (beMatrixTM LS-H high bloom, Nitta Gelatin NA Inc, endotoxin count $<10$ endotoxin units (EU)/g). Factor XIII $(200 \mathrm{U} / \mathrm{mL})$ was first activated with $20 \mathrm{U} / \mathrm{mL}$ thrombin (Baxter) in the presence of $2.5 \mathrm{mM} \mathrm{CaCl} 2$ for $15 \mathrm{~min}$ at $37^{\circ} \mathrm{C}$, and was stored at $-80^{\circ} \mathrm{C}$ until use (FXIIla).

\subsubsection{CaP granule preparation}

Commercially available 35/65\% HA/ $\beta$ TCP CaP granules (Kuros Biosciences) with a submicron surface topography $(\mathrm{CaP} 1)$ or micron topography $(\mathrm{CaP} 2)$ were incubated in EGM-2 medium for 1-2 hours in a humidified incubator $37^{\circ} \mathrm{C}$. Medium was aspirated and granules were left to dry before adding to the hydrogel mixture. Data shows CaP1 type granules, unless indicated otherwise.

\subsubsection{Hydrogel co-cultures with CaP granules}

Embedding MSC/ECFC/CaP in gelPEG: Gelatin-PEG (4:1, 2\% w/v) hydrogels were prepared in tris-buffered saline (TBS, pH 7.6, $40 \mathrm{mM}$ ) with $50 \mathrm{mM}$ calcium chloride and $5 \times 10^{6} \mathrm{MSC}$ and $1.25 \times 10^{6}$ ECFC per $\mathrm{mL}$ hydrogel. Hydrogel crosslinking was initiated upon addition of $10 \mathrm{U} / \mathrm{mL}$ activated factor XIII and droplets of 20 or $75 \mu \mathrm{L}$ were place in the center of a pre-warmed 48 or 12-wells plate respectively. The constructs were left to crosslink for 1 hour in a humidified incubator $37^{\circ} \mathrm{C}$. In case of CaP-hydrogel cultures (gelPEG-CaP+), CaP granules were added to the droplet before gelation.

Culturing of gelPEG-CaP constructs: Constructs were either cultured in i) freshly prepared osteogenic differentiation medium (ODM), composed of MSC expansion medium (without bFGF) supplemented with $10 \mathrm{mM} \beta$-glycerophosphate (BGP) (Sigma), and $10 \mathrm{nM}$ dexamethasone (Sigma), ii) EGM or iii) MSC expansion medium (without bFGF). To check for mature osteoblast cell function and monitor the status of mineralization over time, 20 $\mu \mathrm{M}$ xylenol orange (XO) tetrasodium salt (33825; Sigma-Aldrich) was added to the media.

Embedding MSCs/ECFCs/CaP in Matrigel: Samples were prepared by mixing $35 \mu \mathrm{l}$ ODM, containing both cell types, with $35 \mu \mathrm{l}$ Matrigel on ice and pipetting the mixture $(75 \mu \mathrm{L})$ into a pre-warmed 12 -well plate. The mix was allowed to form a hydrogel at $37^{\circ} \mathrm{C}$ for 1 hour. In case of cultures with CaP granules (Matrigel-CaP+), granules ( 14 mg) were added per hydrogel droplet before gelation. Constructs were cultured in ODM for 14 or 21 days. 
To check if Pi could be involved in the vascular degradation via certain transport channels, they were blocked with phosphate channel blocker phosphonoformic acid (PFA) (1 mM) in $\mathrm{CaP}+$ and $\mathrm{CaP}$ - Matrigel co-cultures (Pilot experiment, $\mathrm{N}=1$ with $\mathrm{n}=4$ samples per group). Hydrogels were cultured in ODM for 3 days to allow for the formation of a vascular network before supplementation of PFA.

Follow up: Media was refreshed 2 times a week, up to day 10 to evaluate the vasculogenesis, or day 14 or 21 to monitor osteogenesis. Pre-vascular network formation (ECFC-GFP, vascular network length quantification) and matrix mineralization (bright field and live imaging of XO, RFP channel) were monitored every day on an Evos FL cell imaging system (ThermoFisher). Constructs were fixed with formalin (10\%) and cut into pieces for ALP staining, paraffin embedding and whole mount immunofluorescent confocal imaging (Leica SP8x).

\subsubsection{Dissolution products of CaP ceramics}

The influence of the dissolution products of the $\mathrm{CaP}$ granules on vascular regression was tested in three ways. First, the effect of the released products from the granules into the medium was tested. GelPEG hydrogel co-cultures ( $75 \mu \mathrm{L}$ droplets) were prepared as described in section 3.3.3, and CaP1 granules were placed around the gel in various amounts in ODM: CaP- (no granules, control), CaP 1x (same amount CaP as in gelPEG-CaP+ construct), CaP 2x (double amount) and CaP-full (4 times the amount, to completely fill the surrounding surface).

Second, the effect of the individual release products in medium was tested. GelPEG hydrogel co-cultures ( $20 \mu \mathrm{L}$ droplets) were prepared as described in section 3.3.3 and cultured in ODM for 3 days to allow a vascular network to form. Hereafter, the ODM was supplemented with either $\mathrm{CaCl}_{2}(2.5 \mathrm{mM}), \mathrm{NaH}_{2} \mathrm{PO}_{4}(3 \mathrm{mM})$ or a combination thereof (on top of the ODM baseline concentration $\mathrm{CaCl}_{2} 1.8 \mathrm{mM}$ and $1 \mathrm{mM} \mathrm{NaH}_{2} \mathrm{PO}_{4}$ ).

Third, the effect of the individual release products within the hydrogel was tested. GelPEG hydrogel co-cultures ( $20 \mu \mathrm{L}$ droplets) were prepared as described in section 3.3.3; within the hydrogel formulation either $150 \mathrm{mM} \mathrm{CaCl}_{2}$ or $3 \mathrm{mM} \mathrm{NaH}_{2} \mathrm{PO}_{4}$, or a combination thereof, was mixed and left for gelation (gelPEG baseline content of $50 \mathrm{mM} \mathrm{CaCl}$ at the time of gelation). For all experiments of 3.4.4. there was a total of $\mathrm{N}=3$ independent replicates with triplicates $(n=3)(N=2$ MSC donor $1, N=1$ MSC donor 2$)$.

\subsubsection{Intracellular calcium staining with FLUO-4}

A Fluo-4 calcium imaging kit (F10489, ThermoFisher) was used for the detection of calcium influx in live cells, according to the manufacturer's protocol. In short, gelPEG$\mathrm{CaP}+$ constructs were co-cultured for 3 days in ODM, and hereafter incubated with ODM 
supplemented with the powerload (1:100), probenectin (1:100) and Fluo-4 (1:1000) solutions. Constructs were incubated overnight at $37^{\circ} \mathrm{C}$ to allow for diffusion into the hydrogel and washed twice with PBS. Fresh ODM with background suppressor (1:10) was placed on the constructs, and constructs were immediately used for live-cell imaging (Leica SP8x).

\subsection{Vessel formation}

\subsubsection{Quantification of the pre-vascular structures}

To evaluate the development of the vascular network in the samples, GFP-ECFCs were visualized on an Evos FL cell imaging system (ThermoFisher). The influence of the CaP dissolution products on the angiogenic properties (section 3.3.4) was further assessed by quantifying the total length of the GFP-structures in the images (length in pixels). On days 3, 5, 7, 10 after encapsulation, images were taken at 3 locations per sample $(n=3$ samples) and processed in Adobe Photoshop CS6. The images were converted to black and white, inverted and brightness was set to -150 and contrast to 100 . The resulting files were batch processed in the freeware program Angioquant [251]. All images had individually optimized processing settings for smoothening, segmentation with automatic thresholding, and pruning of structures below 10 pixels. The total length of the pre-vascular structures (averaged value of the 3 images per sample) was assessed.

\subsubsection{Immunofluorescent a-SMA staining}

Network maturation was evaluated with a-smooth muscle actin (a-SMA) staining to detect mural-like cells, visualized by confocal microscopy (Leica SP8x). In short, after fixation, samples were cut into pieces and used for whole mount immunofluorescence staining. After permeabilization (triton-X 0.2\%, 15 minutes), nonspecific protein binding was blocked for 30 minutes in 5\% PBS-BSA and followed by 1 hour incubation with the anti a-SMA fluorescently labelled antibody (Clone 1A4, Cy3 $0.5 \mu \mathrm{g} / \mathrm{ml}$, C6198, Sigma Aldrich). Finally, nuclei were counterstained with 4',6-diamidino-2-phenylindole dihydrochloride (DAPI) staining $100 \mathrm{ng} / \mathrm{ml}$ (Sigma) for $30 \mathrm{~min}$ at room temperature.

\subsection{Osteogenic differentiation}

\subsubsection{Alkaline phosphatase (ALP)}

ALP was used to indicate early osteogenesis. For the detection of ALP activity, samples were stained within 7 days after fixation. Constructs were washed $3 \times 5$ minutes in Trisbuffered saline (TBS), followed by 15 minutes of permeabilization with TBS-triton $0.1 \%$ with subsequent washing steps of $3 \times 5$ minutes. The ALP substrate (K062511, Fuchsin substratechromogen system, Dako) was prepared according to manufacturer's protocol and incubated for 1 hour on the samples. To stop the reaction, samples were washed in Milli-Q (MQ) water and immediately used for imaging on a stereo microscope (Olympus SZ61). 


\subsubsection{Osteonectin (IHC)}

Osteogenic differentiation was determined via osteonectin immunohistochemistry, a marker for osteoblast differentiation. Before paraffin embedding, CaP samples were decalcified for 3 days in $10 \%$ ethylenediaminetetraacetic acid (EDTA)-PBS solution ( $\mathrm{pH}$ 7.4), dehydrated in a graded ethanol series (70-100 \%) and transferred to xylene. Samples were then embedded in paraffin-wax and sectioned into $5 \mu \mathrm{m}$-thick slices on a microtome. Slides were deparaffinized with xylene and gradually rehydrated through an ethanol series (100-70\%) to demi water before starting the IHC staining.

Peroxidase blocking was performed before the sections were boiled in citrate buffer (10 mM, pH 6, 80 $\mathrm{C}$ ) for $20 \mathrm{~min}$. After protein blocking (15 min, 5\% BSA/PBS), slides were incubated with the primary anti-ON antibody ( 9 $\mathrm{ug} / \mathrm{ml}, \mathrm{DSHB}, \mathrm{AON}-1$ was deposited to the DSHB by Termine, J.D. (DSHB Hybridoma Product AON-1)) and incubated at $4^{\circ} \mathrm{C}$ overnight. Sections were washed $3 x$ for 5 min with PBS-Tween, and incubated with an anti-mouse HRP-labelled polymer for 30 minutes (Brightvision poly HRP-Anti-Rabbit lgG, Immunologic). Addition of DAB substrate resulted in a brown staining and nuclei were counterstained with Mayer's hematoxylin (Merck), dehydrated and mounted with DEPEX.

\subsection{Statistical analysis}

Statistical analyses were performed with GraphPad Prism 6.01. Angioquant outcomes (normalized to day 3 ) were tested for the significant increase or regression of the vascular length over time with a two-way ANOVA repeated measurements (or a fitted mixed model in case of missing values) with multiple comparisons of the mean over time within one condition. A Šidák's test was used to correct for multiple comparisons. Differences between the experimental groups were considered statistically significant if $p<0.05$. Outcomes are presented as means \pm standard deviation.

\section{Results}

\subsection{Confirmation of vasculogenic and osteogenic differentiation of hydrogel co- cultures}

To realize the formation of a pre-vascular network in an osteogenic tissue and to study the effect of $\mathrm{CaP}$ ceramics on vasculogenesis, hemispherical hydrogel constructs were made from gelPEG, with (+) or without (-) CaP granules, and cultured in an osteogenic environment. The model supported vasculogenesis, with the vascular structures adjoined by mural-like cells visualized by a-SMA staining after day 5 (Figure $6.1 \mathrm{~B}$ ). The a-SMA-/ GFP- cells in the co-culture were presumably committed to the osteogenic lineage (Figure 6.1 B, small arrows). Indeed, simultaneously, the constructs showed ALP activity after one week, indicative for osteogenic differentiation (Figure 6.1 C). To further confirm osteogenic differentiation, osteonectin expression was evaluated after 7 days. Both the 
cells and matrix were found positive for this osteoblast marker, especially in the vicinity of the CaP granules (Figure 6.1 D). Matrix mineralization was confirmed after 21 days by the increase of xylenol orange (XO)-positive cells and matrix, indicating calcium deposition (Figure 6.1 E).

\section{A Co-culture model}
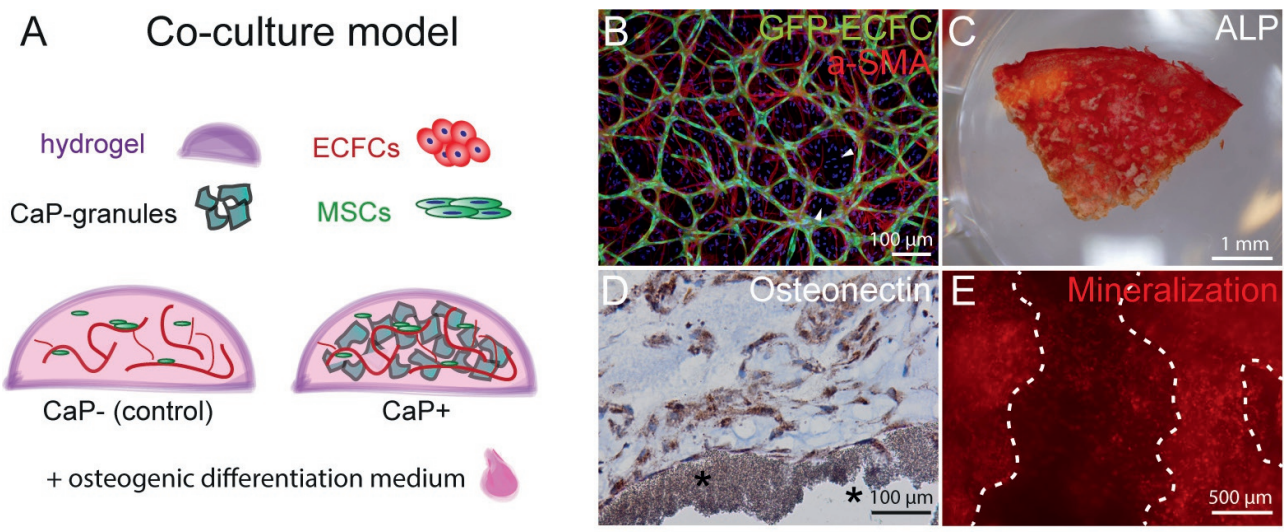

Figure 6.1. In vitro co-culture model for pre-vascularization and osteogenic differentiation of cell-laden CaP gelatin-PEG hydrogels. A) Hydrogels contained a co-culture of endothelial colony forming cells (ECFCs) and multipotent mesenchymal stromal cells (MSCs), with or without the addition of calcium phosphate (CaP) granules, and were cultured in osteogenic differentiation medium. B) A pre-vascular network (GFP-ECFCs, green) was supported by a-SMA positive cells, as soon as 5 days after culture. C) Alkaline phosphatase (ALP) activity (red) after 7 days of culture. D) Immunohistochemistry staining of osteonectin (brown staining) after 7 days of culture. * represents remnants of the granules. E) Calcium depositions, visualized with xylenol orange (XO, in red), indicate matrix mineralization on top of the granules ${ }^{*}$ ) and between them, after 21 days. The dotted white lines represent the outline of the granules.

\subsection{The in vitro vasculogenic network is influenced by the CaP granules}

$\mathrm{A}$ vascular network had already developed by day 3 in both the $\mathrm{CaP}$ - and $\mathrm{CaP}+$ cultures and was maintained till day 7 (Figure 6.2 A). However, a regression of the vascular network was observed in the $\mathrm{CaP}+$ group after 7 days of culturing, where the vascular structures specifically deteriorated near the granules (Figure 6.2 B, images shows the same location over time). Mural-like cells were characterized by a disorganized structure around the vessel remnants and an accumulation of calcium in the deteriorated vascular structures was observed (white arrows) (Figure 6.2 C). In addition to that, fragmented nuclei and cytosolic accumulation of (GFP) proteins in micro-vesicles were observed at these specific locations of degradation (Figure 6.2 D). The cells within the remaining vessel structure appeared to be apoptotic (Figure 6.2 E, YOPRO staining), whereas the nuclei of nonendothelial cells did not show such effects. Apoptosis was also confirmed by positive caspase-3 staining in the vicinity of the CaP granules (Figure 6.2 F, black arrow). 


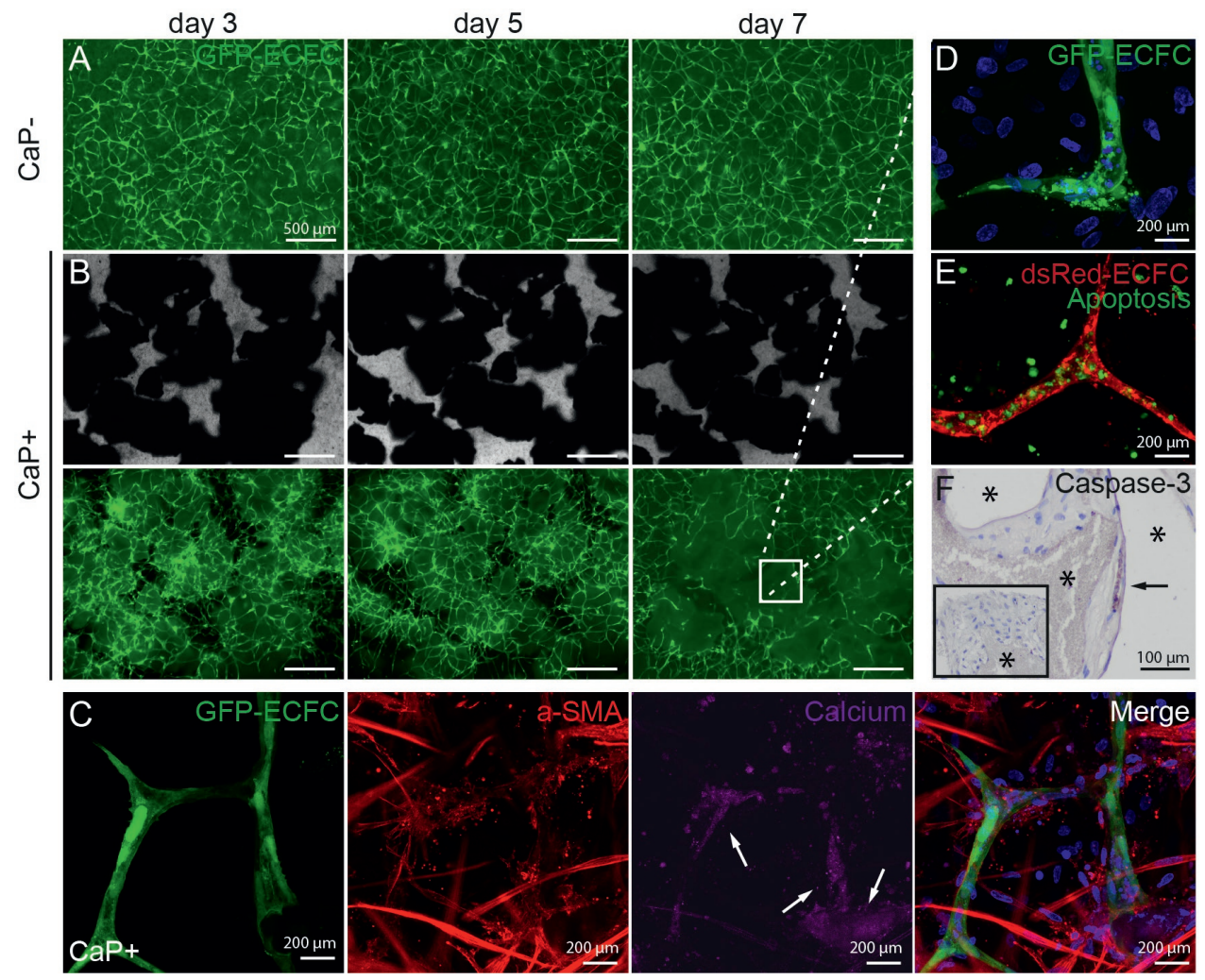

Figure 6.2. Effect of CaP granules on the pre-vascular structures in cell-laden gelatin-PEG hydrogels. A pre-vascular network (GFP-ECFCs, green) was developed after 3 days of culture, both in the A) $\mathrm{CaP}-$ and $\mathrm{B}) \mathrm{CaP}+$ hydrogel constructs. The dark structures in the bright field image represent the granules. Images show the same location over time. C) Immunofluorescent staining for a-SMA cells (red) and xylenol orange (XO, purple) (representing calcium) was observed in the deteriorated vessel structure (white arrows) after 7 days. D) A magnification from B) showing the nuclei in blue (DAPI). E) A high incidence of apoptotic cells (YOPRO, green) was found within the remaining vessel structures (dsRED). F) An immunohistochemical staining for caspase-3 shows apoptosis on paraffin slides. * represents the ghost of the granule and the inset shows isotype staining.

The same vascular regression was observed when i) another type of CaP ceramics with a different surface topography was introduced (CaP2) in the co-cultured hydrogel (Figure 6.3 A) and ii) when the CaP ceramics were cultured in another type of hydrogel (Matrigel) (Figure 6.3 B). In addition, similar results were observed when iii) changing the culture medium from ODM to MSC expansion medium (data not shown) and EGM (Figure 6.3 C). The vascular structures contained an elevated concentration of calcium prior to the actual vessel degradation, independent of the type of medium (Figure 6.3 D-E, white arrows). The cultured constructs in ODM also showed cells positive for calcium deposition at non-vascular locations, which can be explained by the co-occurrence of osteogenic differentiation induced by the ODM, inducing the MSCs to participate in tissue 
mineralization (Figure 6.3 E, smal arrows). With the FLUO-4 indicator it was confirmed that the calcium accumulation was intracellular (Figure 6.3 F).

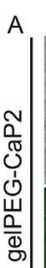

day 3
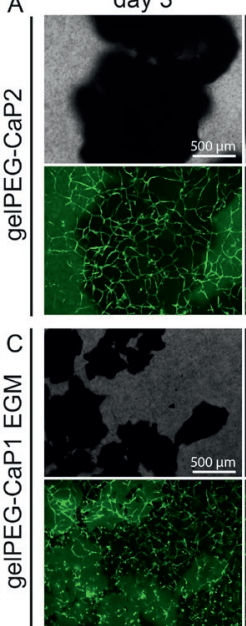

day 5
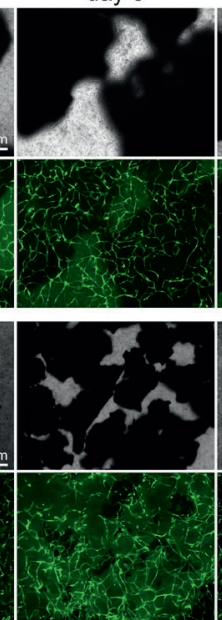

day 7


B day 3

day 5
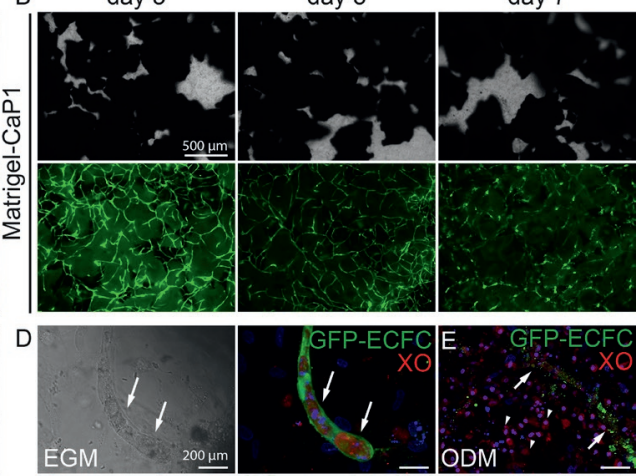

$\mathrm{F}$



Figure 6.3. Adverse effect on the pre-vascular structures occurs irrespective of type of CaP granule, culture medium or hydrogel. Vascular regression was observed after 7 days when A) supplementing the hydrogel with the CaP type 2 granules, B) culturing the CaP type 1 granules in Matrigel or when C) culturing the gelPEG system with CaP1 granules in endothelial growth medium (EGM). D) Prior to degradation, vessels were observed to accumulate calcium (XO, represent calcium), both in EGM cultured constructs and E) in ODM cultured constructs. In the ODM cultures, calcium deposition was observed throughout the whole gel and not only the sites of vascular regression (white arrow heads). White arrows indicate the vessel structure on both bright field and fluorescent images. F) The calcium accumulation (FLUO-4, green) in the vessel structures (dsRed) was intracellular.

\subsection{Addition of inorganic phosphate to the culture system impacts vascular network}

To determine if the released factors from the CaPs, rather than a contact-dependent effect, was responsible for the vascular degradation, the CaP granules were added to the medium instead of the hydrogel. The addition of CaP granules to the medium had no negative effect on the vascular length over time (Figure 6.4 A-B). However, at the edge of the construct, where cells were in contact with the granules, apoptotic cells were clearly visible (inset). When high concentrations of the individual dissolution ions calcium and $\mathrm{Pi}$ were added to the culture medium, significant vascular network regression occurred in the $\mathrm{Pi}$ and calcium/Pi conditions (Figure 6.4 D-E), $\mathrm{Pi}(\mathrm{p}<0.001)$ and $\mathrm{Ca} / \mathrm{Pi}$ (day 5 vs day 10 $\mathrm{p}<0.0002$ and day 7 vs day $10 \mathrm{p}<0.049$ ). 



B
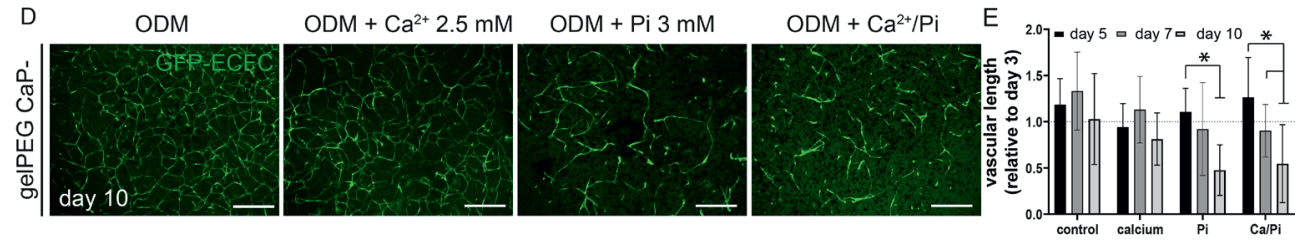

Figure 6.4. Influence of $\mathrm{CaP}$ dissolution products on the vasculature. A) $\mathrm{CaP}$ granules were added in different amounts (none; the amount as placed in a $\mathrm{CaP}+$ hydrogel (CaP 1x); double the amount; and a full well (4x)) to the ODM that was surrounding a co-culture construct. Inset shows apoptosis (YOPRO, green) at the edge where the granules touched the hydrogel). B) Quantification of the length of the vascular network. The vascular length of the CaP- group decreased significantly after 10 days $(p<0.02)$. The graph shows length in pixels relative to day 3 (dotted line, values above 1 represent an increase), for $\mathrm{N}=3$ independent experiments, with triplicates. C) Bright field image after 10 days of culturing, calcified nodules (bright field) co-localize with XO (red, inset). D) Fluorescent images of $\mathrm{CaP}$ - hydrogel constructs. After 3 days the ODM was supplemented with $2.5 \mathrm{mM}$ calcium, $3 \mathrm{mM}$ phosphate (Pi) or a combination thereof. The calcium group followed the trend of the control groups, whereas the vascular network disappeared in the groups with $\mathrm{Pi}(\mathrm{p}<0.001)$ and $\mathrm{Ca} / \mathrm{Pi}$ (day 5 vs day $10 p<0.0002$ and day 7 vs day $10 p<0.049$ ).

This effect was especially visible when the individual dissolution factors were incorporated in the gelPEG hydrogel. The addition of extra $\mathrm{CaCl}_{2}$ alone did not show a specific influence on the morphology and length of the vascular network after 7 days (Figure 6.5 A-B), and followed the same trend as the control situation (Figure 6.5 E). On the other hand, the addition of Pi triggered network degradation in a similar trend as observed when $\mathrm{CaP}$ were embedded within the hydrogel (Figure 6.5 C), meaning that a vascular network was initiated, but regressed afterwards. GelPEG with the calcium and Pi combined resulted in the formation of few vascular structures which were almost completely degraded after 7 days (Figure 6.5 D), also confirmed by the quantification of the vascular network over time (Figure 6.5 E). Both the groups with Pi and calcium/Pi added, contained crystal precipitation in the matrix, with the highest occurrence in the calcium/Pi group. Depositions were visualized by the dark matrix in the bright field images and stained positively for XO (Figure 6.5, insets), especially bright in the calcium/Pi group. Moreover, ALP activity was decreased (qualitatively assessed) in the $\mathrm{Pi}$ and the calcium/Pi supplemented gels (Figure $6.5 \mathrm{~F})$. 



Figure 6.5. Effect of the addition of calcium and/or Pi to the gel in a cell-laden gelPEG CaP-hydrogel. A) gelPEG CaP- hydrogel co-culture showed the formation of a vascular network (ECFC-GFP, green) when cultured in ODM. B) fluorescent images and bright field images of hydrogels supplemented with $150 \mathrm{mM}$ calcium, C) $3 \mathrm{mM}$ Pi and D) $150 \mathrm{mM}$ calcium/3 mM Pi. Inset show XO in red, representing calcium signal. E) Graph shows the quantification of the length of the vascular network in pixels relative to day 3 (dotted line, values above 1 represent an increase), for $\mathrm{N}=3$ independent experiments, with triplicates. Addition of $\mathrm{Pi}(\mathrm{p}<0.0013)$ and $\mathrm{Pi} / \mathrm{Calcium}(\mathrm{p}<0.0001)$ resulted in significant decrease of the vascular length. F) ALP activity in the hydrogel constructs after 7 days.

\subsection{Calcification of vessels occurs in hydrogels without CaP granules}

When the osteogenic differentiation period of the CaP- hydrogels (both in gelPEG, gelPEG + Ca2+ and Matrigel) was pro-longed to 10 days, another event was observed: calcified nodules appeared in the shape of multinodular vesicles (Figure 6.6 A). These nodules were positive for calcium and specifically co-localized with the vascular structures (white arrows), and were confined to the lumen of the vessels (Figure 6.6 B). At the site of those calcified nodules, the nuclei were fragmented and cells were apoptotic (Figure 6.6 C), and also showed disorganization of a-SMA ${ }^{+}$structures (Figure 6.6 D). These calcified nodules were most pronounced in the CaP-co-cultures and appeared consistently after 10 days of osteogenic culture, correlating with significant vascular network regression (Figure 6.4 A-C). Interestingly, none of the gelPEG CaP- cultures with the CaP granules added to the surrounding medium, showed formation of calcified nodules (Figure 6.4 C). When the culture time was extended to 21 days, the matrix of all groups started to mineralize, characterized by the darkening of the matrix and XO deposition, which was different in appearance compared to the calcified nodules. The observed calcification of the vessels could be blocked by the use of the phosphate channel blocker PFA, and no calcifications were found when cultured for up to 21 days (Supplementary Figure 6.1, preliminary data $\mathrm{N}=1, \mathrm{n}=4$ ). The addition of PFA did not appear to influence the ALP activity of the constructs, based on qualitative observations (Supplementary Figure 6.1 C). Nevertheless, 
the addition of PFA was not successful in overcoming the problem of the vascular regression in the $\mathrm{CaP}+$ cultures (Supplementary Figure 6.1 A-B).


Figure 6.6. Calcification in pre-vascular structures in cell-laden CaP-hydrogel constructs. A) Bright field images of hydrogel cultures after 10 days in ODM. Nodules (white arrows) and XO-positive signal (red, representing calcium) co-localize. B) The nodules appeared specifically within the vessels, confirmed with the cross-sectional visualization at the right hand side. C) Nodule formation (white arrow), observed with bright field imaging, co-localized with the presence of apoptotic cells (green, YOPRO). D) a-SMA (red) irregularities (arrow head) and fragmented nuclei (white arrows) were observed at the sites where calcifications appeared. Scale bar represent $200 \mu \mathrm{m}$.

\section{Discussion}

We previously reported adverse effects of CaP granules on in vitro vasculogenesis (chapter 5), and this study was set up to determine the underlying cause. MSCs and ECFCs were encapsulated in either a gelPEG hydrogel [294] or Matrigel [206], with or without the addition of $\mathrm{CaP}$ granules $(35 / 65 \% \mathrm{HA} / \beta \mathrm{TCP})$ and cultured in several media types. The model provided insight in the factor(s) of the CaP ceramics that caused the in vitro vascular network degradation, where the inorganic phosphate (Pi) dissolution product appeared to be the dominant factor. Moreover, in this chapter, a second event that induced vascular regression was observed, specifically in hydrogels without CaP granules. Here, vascular calcifications in the shape of calcified nodules within the pre-vascular structures were observed. This pathology also coincided with the addition of a phosphate source, which is a standard component of osteogenic differentiation medium for MSCs. Additionally, we provided preliminary data on how to resolve the vascular regression by blocking a phosphate channel, which gives a starting point for further development of the study.

Under normal conditions, mineralization is a tightly regulated process in which osteoblasts hydrolyses extracellular pyrophosphate (PPi) into Pi that, together with calcium, is transported in microvesicles (MVs). Once released, these MVs serve as nucleation sites and continue to accumulate $\mathrm{Pi}$ and calcium to stimulate the formation of HA crystals. 
Maintaining a stable PPi/Pi homeostasis is crucial for the regulation of normal mineral deposition, and a slight misbalance can result in various pathological responses, such as cell apoptosis, skeletal diseases or ectopic ossifications [309]. Our experimental results suggest that the addition of an osteogenic stimuli (i.e., addition of CaP granules or the use of osteogenic medium) has the potential to cause an imbalance in the PPi/Pi homeostasis, which can negatively influence the vasculature in the in vitro hydrogel culture.

In the CaP-containing constructs, apoptosis was the underlying pathology of the vascular regression. It is speculated that the induction of apoptosis was caused by a specific surface reactivity between the CaP granules and the hydrogel and/or accumulation of the release products, due to limited ionic exchange through the hydrogel in this static in vitro set-up. This could distress the cells after prolonged exposure to the ions or after exceeding a threshold concentration. The effect was shown to be independent of the surface structure of the CaP ceramics, since both the CaP1 (submicron surface topography) and the CaP2 type (micron surface topography) initiated a vascular regression. Nevertheless, a one to one comparison of both types of ceramics is complicated since the sintering process, required to adapt the surface topography, also affects the chemical composition and indirectly the rate of resorption.

The in vitro and in vivo resorption of $\mathrm{CaPs}$ is a complex process that greatly depends on its physicochemical dissolution, which is influenced by the solubility of the material and $\mathrm{pH}$ of the surrounding environment. In general, cell culture environments range between a $\mathrm{pH}$ of $7.0-7.4$, whereas degradation of CaP bioceramics normally occurs in an acidic environment that is facilitated by, e.g., osteoclasts, therefore making predictions on resorption reactions in a hydrogel system a complex matter. Moreover, the resorption also varies a lot between chemical composition (e.g., type of ceramic and $\mathrm{Ca} / \mathrm{P}$ ratio) and topography (e.g., porosity and surface area) of the material, as well as the kinetics of the surrounding milieu (e.g., solution, hydrogel or tissue) [310]. When examining the components in our system, the dissolution products will be presumably derived from the relatively soluble tri-calcium-phosphate $(\beta-T C P) \mathrm{Ca}_{3}\left(\mathrm{PO}_{4}\right)_{2}$ component rather than from the less soluble hydroxyapatite $(\mathrm{HA}) \mathrm{Ca}_{10}\left(\mathrm{PO}_{4}\right)_{6}(\mathrm{OH})_{2}$ part [311]. To address the influence of the biphasic composition in our model, future research could for example include CaP granules with various ratios of HA/ $\beta$-TCP. This set-up could help to determine if the released products from the $\beta$-TCP component are indeed the causing factor that interferes with the maintenance of capillary-like networks.

If the entire reaction if simplified, and assuming dissolution will take place, the products released will be calcium ( 8 ppm after 3 hours in simulated physiological saline, SPS, data kindly provided by the manufacturer) and inorganic phosphate (Pi) (release profile unknown, ongoing work). Together, these ions can cause a local increase in ion 
concentration, surface charge and $\mathrm{pH}$ within the construct or culture medium [311, 312]. We further investigated which of these factors instigated the decrease in vascular survival in the $\mathrm{CaP}+$ cultures. An increase in intracellular calcium was observed specifically in the degrading apoptotic vascular structures. The high calcium concentration could either be the cause of the vessel degradation, as an excess of cellular calcium is highly toxic to cells (e.g., ECs and vSMCs) and can lead to apoptosis due to a calcium mitochondrial overload [313-315]. On the other hand, it could also be the result of the induction of apoptosis by other extracellular events, which consequently induces mitochondrial dysfunction and results in calcium release from mitochondria into the cytoplasm [303, 314, 315]. When tripling the calcium concentration in the gel or culture medium (exceeding the measured release profile of the CaP ceramics), no effect on vascular degradation was observed up till 7 days. This suggests that the increased calcium influx was more likely a downstream effect rather than the actual cause of the vascular degradation.

Contrarily, supplementing the culture environment with an excessive concentration of $\mathrm{Pi}$ (matched to circulating phosphate concentrations in hyperphosphatemia (>2.0 mM) [316, 317]) induced the same trend as when cultured with the CaPs, i.e., the initial development of a vascular network followed by degradation of the structures. Thus, out of all assessed parameters, only the addition of $\mathrm{Pi}$ resulted in vascular regression resembling that when cultured with CaPs, either individually or in combination with calcium. This is in agreement with observations made by diMarco et al., where high extracellular Pi levels $(2.5 \mathrm{mM})$ induced endothelial cell apoptosis while exposure to high calcium concentration ( $2.8 \mathrm{mM})$ alone did not. Additionally, they reported increased apoptosis rates with the synergistic effects of calcium and $\mathrm{Pi}$, associated with disruption of mitochondrial membrane potential, reactive oxygen species (ROS) generation and caspase activation. High levels of Pi ( $2.5 \mathrm{mM}$ and $5 \mathrm{mM}$ ) are also involved in impairing micro particle shedding, downregulating angiogenesis and occlusion of capillaries [304]. In our set-up, CaP depositions could be seen in the condition with only $\mathrm{Pi}$ in the gel. This was possibly attributed to the baseline levels of calcium in the gelPEG system and in the medium, with which the Pi could react and precipitate. Therefore, it is perhaps inaccurate to relate the apoptosis of cells solely to the presence of $\mathrm{Pi}$, as in the end it was not only present individually but also in the shape of CaP crystals.

It remains unclear why no effect was found when the CaP granules themselves were submerged in the medium. Possibly, the concentrated dissolution products from the biomaterial were removed with every medium change, preventing the buildup of resorption products in the medium. Furthermore, it is known that the dissolution products of CaP ceramics can form a new phase at the surface of the material [318] and in this process calcium and $\mathrm{Pi}$ can be captured from the surrounding medium or hydrogel as well. This process could explain why the CaP granules in the medium appeared to have a protective effect on the formation of calcified nodules within the capillaries of CaP- 
hydrogels. This represents the second observed cause of degradation of the vascular network in this study: calcified nodules within the capillary-like structures.

The formation of nodules shares a similar pathology to vascular calcification (VC), which has a high incidence rate in patients with chronic kidney disease (CKD). VC is a condition that has many similarities to bone ossification but is described as a cell-mediated process of HA crystal deposition at non-ectopic locations, mostly occurring in the tunica intima or tunica media of larger vessels [319], and is less described in capillaries [303, 304, 320]. Clinical and epidemiologic studies consistently show that high circulating phosphate (hyperphosphatemia) and calcium (hypercalcemia) levels in patients are associated with VC. There is also strong evidence that endothelial dysfunction is involved in this pathology, but research has mostly been focused on the vascular smooth muscle cells (vSMCs) [303, 304, 315, 321-323].

In our system, disturbing the PPi/Pi homeostasis by the supplementation of increased levels of increased levels of phosphates in the medium could have triggered a calcification process that is similar to VC. To induce osteogenic differentiation in in vitro cultures, the phosphate source ( $\beta$-glycerophosphate (BGP)) for conversion into $\mathrm{Pi}$ is supplemented to account for osteoblast differentiation and mineralization [324]. However, while osteogenic cells require high levels of phosphate to carry out their function, it seems to dysregulate the vasculogenic cells.

Vasculogenic cells are responsive to high Pi levels through the presence of the sodium phosphate (NaPi) type III channels (Pit1/2) on the cell membranes, which were previously shown to play a major role in the calcification process that takes place in vSMCs and ECs [309, $325,326]$. In our system, the NaPi type II blocker PFA (foscarnet) was able to block nodule formation within the vessels, confirming that it was caused by the supplementation of an Pi source. Nevertheless, the effect of PFA was more likely to be related to its resemblance to PPi or bisphosphonate drugs rather than its ability to block the NaPi channels, since ECs and VSMCs in general do not express type II channels [326, 327].

The PFA-treated construct still contained active ALP, in similar amounts as observed in nontreated constructs, which suggests that the early osteogenic activity was preserved in the system. However, it has to be noted that the use of a phosphate channel blocker or a PPi analogue can affect osteoblast function in the co-culture model, since osteoblasts express the same $\mathrm{NaPi}$ channel as ECs and the $\mathrm{NaPi}$ transport is indispensable for mineralization [328]. Therefore, progressive osteogenic differentiation in terms of osteoblast differentiation and mineralization in our constructs should be confirmed further to investigate the influence of PFA on osteogenesis. Moreover, using a phosphate channel blocker can affect cellular homeostasis, and PFA addition was found to obstruct the formation of a vascular network in 
our system when added immediately after cellular encapsulation in the hydrogel. Also, the use of PFA did not prevent vascular regression in the hydrogel CaP+ cultures. We postulate that the accumulation of the products was already too high in the vicinity of the granules, and the concentration of PFA was not optimal. Presumably, the duration of the exposure, the co-exposure to the additional dissolution of calcium and Pi from the CaP granules, on top of the BGP in ODM, caused a faster apoptotic response. This resulted in the different pathological characteristics of vessel degradation in the CaP- (i.e., nodule formation) compared to the CaP+ culture groups (i.e., apoptosis).

Nevertheless, the MSC-ECFC gelPEG CaP+ constructs showed high rates of bone formation after in vivo implantation in an ectopic location (chapter 5) and show their feasibility for the translation towards an in vivo situation. For the further conversion of the co-culture hydrogel $\mathrm{CaP}+$ model to the bioengineering of perfusable bone analogues, future studies could focus on altering the culture environment (e.g., type of CaP granules and concentration of differentiation medium), working with phosphatonins that regulate $\mathrm{Pi}$ homeostasis (parathyroid hormone (PTH), FGF-23, or Klotho), blocking EC/ECFC specific phosphate channels, or working with PPi mimicking agents (e.g., bisphosphonates). Also, it should be investigated if the CaP biomaterial stiffness or its pressure on the cells in the hydrogel, rather than the dissolution, is the factor that causes the vascular regression. Moreover, we stress that we have presented a simplified version of the possible dissolution products of the CaP ceramics, which has more complexity when in contact with a hydrogel rather than having a free ionic exchange when being in a liquid.

\section{Conclusion}

The observed vascular regression in the $\mathrm{CaP}+$ constructs, and the formation of calcified nodules in the CaP- hydrogels, could be attributed to high levels of phosphates. From our tested parameters, only the addition of Pi resulted in vascular regression resembling that when cultured with CaPs. Moreover, blocking of the NaPi channels by the use of the PFA (foscarnet) prevented the initiation of vascular calcification in the CaP- constructs. Our results highlight that this co-culture system allows detailed observations on the response of a biological system to biomaterials, and can be used to further improve our bioengineered vascularized bone constructs, as well as the study of new drugs and disease modeling (e.g., drugs for the treatment of VC).

\section{Acknowledgements}

This research was partially funded by an NWO (Netherlands Organization for Scientific Research) Graduate Program Grant (022.005.018). We thank Kuros Biosciences for providing us with the bioceramic materials. Moreover, we thank Florence de Groot and Lizette Utomo for the discussions and input, and Leanne de Silva and Alessia Longoni for technical support. 


\section{Supplementary Figures}

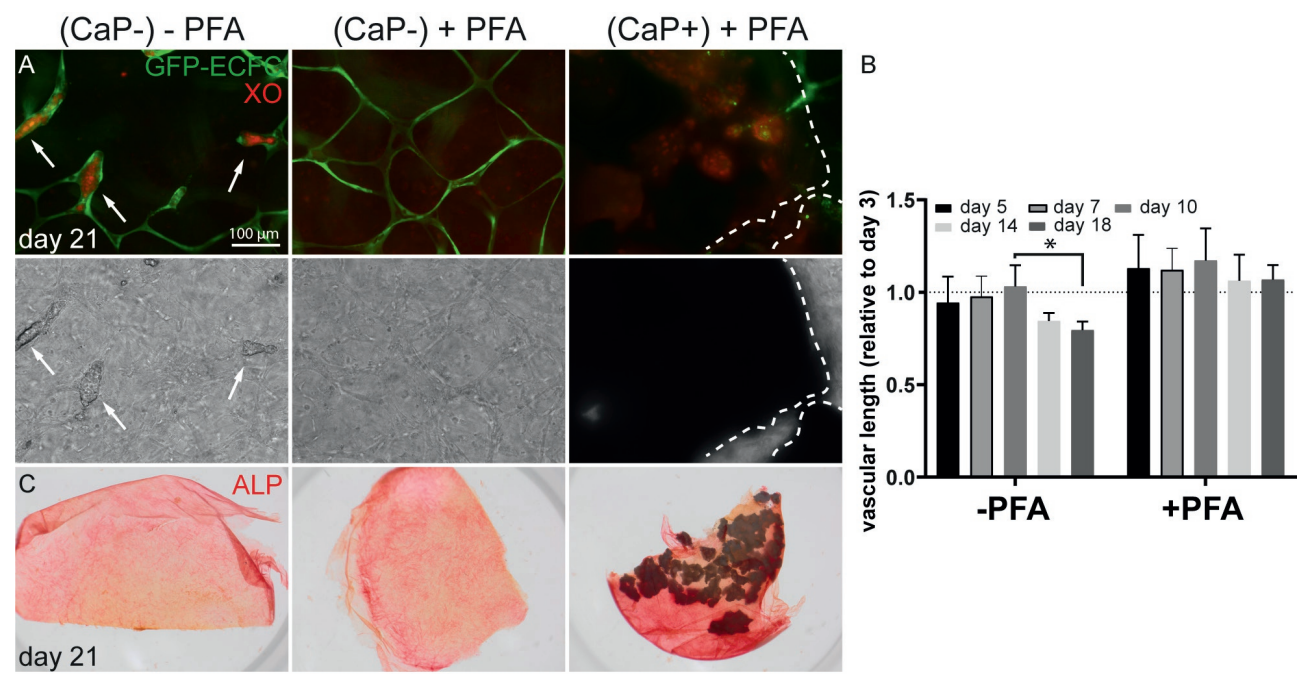

Supplementary Figure 6.1. The phosphate channel blocker phosphonoformic acid (PFA) prevents calcification of capillary structures. Fluorescent images (GFP-ECFC, green; XO, red) of Matrigel A) $\mathrm{CaP}$ - constructs without PFA, CaP- construct with PFA and CaP+ constructs with PFA added to the medium after 3 days. Bright field images show calcified nodules with the white arrows. B) Nodule formation after 10 days resulted in vascular network regression $(p<0.02)$ in Matrigel CaP- constructs. C) alkaline phosphatase (ALP, red) was active in PFA-treated and untreated constructs. 


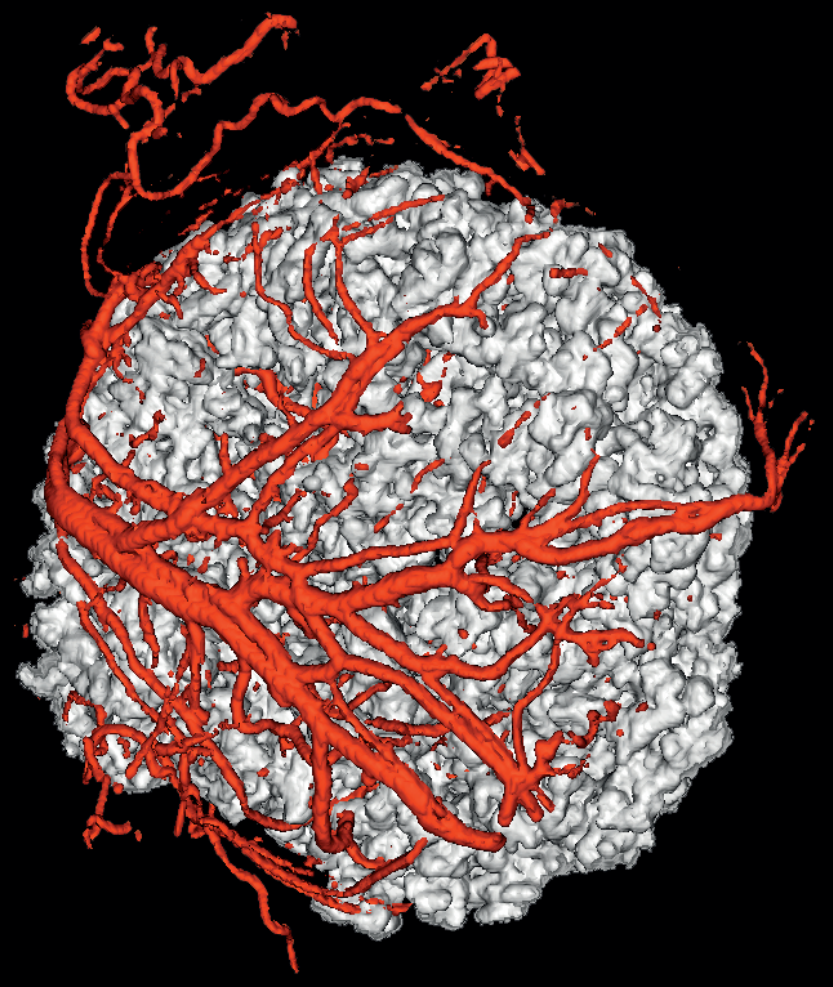


Summary and general discussion 


\section{Summary and main findings}

It was the aim of this thesis to bioengineer a hierarchical vascular supply for the application of pre-vascularized osteogenic tissues that are readily perfusable upon implantation. To this end, we were inspired by the organization of the vasculature in the body, where its shape can be described as a vascular tree. Here, large vessels (macrovasculature $>100 \mu \mathrm{m}$ $\varnothing)$ facilitate blood flow directly from and to the heart. The macrovasculature branches off into the microvasculature, consisting of mesovessels (arterioles and venules, $10-100 \mu \mathrm{m}$ $\varnothing)$, that further disseminate the flow into a microcapillary bed to provide blood to the tissues $(<10 \mu \mathrm{m} \varnothing)$ [33-35]. Inspired by this architecture, we engineered components of the macrovasculature and microvasculature for the bioengineering of bone constructs in vitro. Here, the macroscale vessel would serve as a point of anastomosis to the patients, and the capillaries would facilitate the exchange of oxygen and nutrients with the cells in the bone tissue analogues. At first, we bioengineered a macrovessel by designing a heterotypic scaffold that was inspired by the layered structure of a native blood vessel. To induce further maturation of these neovessels, we developed a bioreactor system that applied shear stress to the luminal side and differentiated the specific cell layers separately. Secondly, osteogenic tissues with capillary-like structures were generated in hydrogel constructs and later on combined with bioceramics to enhance bone formation in an in vivo setting. As an ultimate goal, the two different levels of vasculature should be interconnected in vitro or in vivo to reach the bioengineering of a vascularized osteogenic tissue, with a point of surgical anastomosis for immediate perfusion. In this thesis, we further provided background information on the envisioned in vivo follow-up study to facilitate the in vivo interconnection in an AV-loop model. Moreover, we proposed a technique to mold the microvascular co-culture model around the macrovessel to enable the in vitro interconnectivity.

The main findings of this thesis are:

Chapter 2 Heterotypic scaffold design orchestrates primary cell organization and phenotypes in co-cultured small diameter vascular grafts

- By using a hybrid fabrication approach with controllable parameters, scaffold design criteria can be tailored to the desired biomimetic situation, rather than letting (the limitations of) the biofabrication approach drive the scaffold design.

- The tissue-mimetic layered topology in a heterotypic scaffold design can instruct the formation of a physiological tissue-like organization and differentiation, which is a powerful application for the biofabrication of tissue analogues. 
Chapter 3 Layer-specific cell differentiation in bi-layered vascular grafts under flow perfusion

- Using a bioreactor chamber, that resembles a physiologically relevant environment and provides a compartmentalized exposure of the cell layers to a specific culture media, drives the acquirement of the targeted cell phenotypes (i.e., endothelial cells and vascular smooth muscle cells) and thus the construct functionality.

Chapter 4 Effect of donor variation on osteogenesis and vasculogenesis in hydrogel cocultures

- Induction of vasculogenesis in an osteogenic construct can occur via crosscommunication between endothelial cells and osteogenically induced MSCs. This creates a mutually stimulating environment that influences mural cell differentiation and vasculo-osteogenic processes. It represents the tight coupling of angiogenesis and osteogenesis, as seen in the in vivo situation of bone formation.

- Including cell types from different donors in co-culture models can lead to an imbalance in the vasculo-osteogenic cross-communication. This can result in variations in outcomes of vascular and osteogenic differentiation. The reproducibility and standardization of co-culture protocols and selected release criteria are essential in enabling quality control of the resulting tissue constructs for clinical application.

- For the purpose of tissue engineering, preferably potent autologous cell sources with high proliferative and regenerative capacity should be used. However, application of autologous cell sources is often limited in adults. Induced pluripotent stem cells that are differentiated to endothelial cells (iECs) can serve as a feasible alternative. Still, it is imperative that several important hurdles towards clinical translation are taken when working with this type of cell source.

Chapter 5 In vitro pre-differentiation of pre-vascularized bone constructs containing calcium phosphates accelerates the onset of in vivo bone formation

- Combining a versatile gelatin-PEG based co-culture system with calcium phosphate (CaP) bioceramics enhanced the in vivo bone formation. By altering the length of in vitro construct pre-differentiation, the origin of the bone tissue after implantation can be tuned, which is subject to the retention of the donor cells within the construct.

- Calcium phosphate ceramics can negatively influence the vasculature in an in vitro hydrogel culture. This emphasizes the need for suitable in vitro models to evaluate 
cellular behavior in the presence of biomaterials; especially for predictive models where findings can be extrapolated to an in vivo situation.

Chapter 6 Osteogenic stimulation interferes with the maintenance of capillary-like networks in osteogenic constructs

- The delicate cross-communication of osteogenesic and angiogenic processes in the co-culture model can be disturbed by tipping the balance towards osteogenic induction.

In the following sections, the limitations of our studies, the impact of our findings on the field of regenerative medicine and the future perspectives are discussed.

\section{General discussion}

\section{Functionality of tissue engineered constructs}

Functionality of an engineered construct is important to provide an efficacious implant at the time of implantation, which also will help to withstand the environment that the construct will encounter after surgery [329]. Additionally, some of the tissues that require replacement have an important structural function, such as load-bearing bones or vasomotive control of flow in blood vessels. Recapitulating these biomechanical properties is tightly related to an appropriate in vivo function, and bioengineered constructs should therefore restore these functions to get successful clinical outcomes [330, 331]. To account for these challenges, we should address functionality in tissue engineered constructs (FTE) and define what actual functional success is.

Functionality can roughly be divided in biomechanical and biological functionality. In the field of bioengineering, the biomechanical functionality is largely dependent on the scaffold properties (e.g., design and material), whereas the biological function is reliant on the cellular behavior and phenotype [330,331]. While the mechanical and biological environment may differ between tissue classes, as do the processes for their construct preparation, there are commonalities in their desired outcomes and in the challenges to be faced. In our efforts to generate the ideal engineered construct, several questions remain to be answered: when is a tissue construct actually considered to be ideal, and to what extent should we strive for the perfect tissue mimic and functionality prior to implantation? Also, will the level of in vitro functionality predict the remodeling into the desired tissue once implanted in vivo? In other words, how good is good enough?

In part I (chapter 2 and 3) of this thesis, we focused on the vital points that should at least be included in the design of a macroscale vascular substitute and culture environment 
to translate into a functional vascular graft. Here, we also discuss which hurdles still have to be overcome to reach that goal, which will be elaborated on in the following section. In part II (chapter 4-6) of the thesis, we specifically focused on the in vitro biological functionality of an osteogenic construct with a microvascular network, and how this can influence the outcomes after implantation.

\section{Vascular scaffold design to guide neotissue formation}

To facilitate true regeneration, a vascular graft should have the potential to regenerate into a neovessel with the architecture, biological and biomechanical function similar to those of the native vessel. Central to the biomechanical properties is the scaffold structure (i.e., passive mechanical cues [231]), which can be made from various materials and directed to resemble the native architecture of the vessel. The mechanical properties of a native vessel are mainly derived from the composition and the orientation of the ECM components (mainly collagen type I and III, elastin and proteoglycans) in the different tissue layers of the vessel [60]. Thus, the scaffold design should be similar to the layered structure of the vessel and the native microenvironment, with distribution of the ECM components as seen in the vessel layers. In particular, blood vessels are composed of helically arranged collagen bundles in the tunica media with multiple layers of circumferentially orientated vascular smooth muscle cells (vSMCs), and a dense layer of isotropic collagen fibers in the tunica intima that serves as a basis to form a confluent endothelial monolayer on $[58,131$, $133,332,333]$.

Approaches for creating vascular grafts can rely on several fabrication techniques $[42,112]$ and an increasing number of studies has focused specifically on recreating this distinct layered structure of vessels $[49,61-63,136,143,334]$. A promising technique for this is the processing technique named electrospinning [57-59]. Herein solution electrospinning (SES) is often used for the generation of fibrous scaffolds. SES is a reliable technique for the generation of scaffolds with small fibers (nanometer to micrometer range), beneficial for the development of an endothelial monolayer and inhibition of intimal hyperplasia (i.e., neointimal ingrowth of vSMCs) $[110,122,335]$. Nevertheless, it lacks in specific control over fiber deposition and therefore results in an isotropic structure with only little control over the design. With the constant developments in the field of biofabrication, advanced methods that offer more control over this process are becoming available, such as melt electro writing (MEW) [123, 124, 126, 127]. This processing technology employs spatially controllable polymer melts and thereby offers more control over the location of the fiber deposition and their orientation. This allows for the fabrication of a porous medial scaffold layer for vSMC infiltration with an established orientation, and results in constructs with a well-defined architecture. However, the technology is time-consuming and mostly generates fibers with a magnitude larger diameter. 
Since both SES and MEW have their own advantages and structural properties, combining these techniques holds promise for the creation of advanced hybrid scaffolds. This means that scaffolds could be fabricated with different architectures and materials, which provides the different cell types with their desired substrates and brings the development of heterotypic scaffolds to the next level. Chapter 2 demonstrates the potential of combining, for the first time, SES and MEW for the creation of a heterotypic scaffold design that was tailored to the need of the cell types in the vessel layers. The tissue-mimetic layered hierarchy directed a physiological tissue-like cell organization and differentiation. This indicates that structural properties are not only central to the biomechanical function, but can also guide biological responses with regards to tissue organization and orientation. This further aligns with the findings that mechanobiological interactions between cells and scaffolds can influence cell behavior, and that biological and mechanical functionality can be coupled to each other [231,331]. The control over scaffold design is a powerful tool for in vitro culturing and modelling, and is also considered important for guiding and maintaining the cellular organization after implantation.

\section{Differentiation and maturation for biofunctionality}

Besides resembling the architecture of native vessels, an ideal vascular substitute should also exhibit the biofunctional characteristics of the tissue it will replace [60]. The biofunctionality of a tissue analogue is mostly dependent on the acquired phenotypes and subsequent maturation of the incorporated cells. Differentiation or maturation can be achieved via several ways, including the use of growth factors in the medium [152, 153], incorporation of bioactive moieties [336] or structural cues [182]. Further enhancing this maturation can be achieved by including active mechanical cues to the construct, such as applying cyclic stress or shear stress to the scaffold in a bioreactor system [136, 207, $337,338]$. Here, the hemodynamic loading mimics the (patho)physiological environment in the body, where shear stress is applied by the blood flow and cyclic radial stress results from the pulsations of the beating heart. Indeed, by culturing the engineered vascular grafts in a bioreactor chamber, in which the mechanical loading of the blood flow was recapitulated, the functionality of the endothelium in the construct was enhanced (chapter 3). Additionally, the system provided a compartmentalized exposure of the cell layers to specific culture media, which evolved the phenotype of the multipotent mesenchymal stromal cells (MSCs) towards that of vSM-like cells.

\section{Mechanical properties of scaffold structures to enhance biofunctionality}

To further mature vSMCs in vascular grafts, the scaffold should be able to transmit the dynamic cues of strain and stress to the vSMCs. The cells would then sense these mechanical cues and be further stimulated towards mature vSMCs, to eventually gain full functionality. Functionality of vSMC entails their contractile and vasomotive regulatory properties in response to signaling factors, often derived from the EC monolayer that 
signals to the medial layer to adapt to changes in blood pressure and shear stress [184, 225]. To evolve the functionality of the vSM-like cells in our system, we should further resemble the native mechanical loads by first making use of more compliant materials in the scaffold structure. Compliance is one of the hallmarks in the engineering of a vascular graft; it is a measure for flexibility and is inherent to the vessels ability to sustain the blood pressure and pulsations without rupturing $[60,339]$. The polymer poly $(\varepsilon$ -caprolactone) (PCL) used for our scaffold is less compliant than desirable for vascular grafts, but it is a material very suitable for MEW and currently one of the few materials that can be processed by this technique [340]. At present, this limits the freedom and translation towards the generation of a construct that can be mechanically loaded for the VSMC maturation. To meet these demands, the applicability of other polymers for MEW processing is investigated in our ongoing studies, and layering of different elastic (or fast degrading) polymers with separate processing techniques is explored to further fine-tune the mechanical properties. This would not only improve the compliance of the scaffold, but it also could contribute to achieving other requirements set for the ideal vascular graft (suture retention strength, fatigue strength and prevention of kinking) $[49,60,339]$.

\section{Use of extracellular matrix components for functionality}

The functionality of a bioengineered construct could also be improved by using ECM components in the scaffold composition. The ECM plays a crucial role in regulating growth factor signaling at the cellular level, and the ECM compositions follow the vessel hierarchy and blood flow patterns [341]. Collagen for example is the most abundant matrix component in vessels and accounts for their viscoelastic properties [60]. On the other hand, the structural matrix component elastin is also of importance. In native vessels, elastin provides for the elasticity of the vessel and is found in a membrane-like organization (muscular arteries) or as individual fibers (elastic arteries) in the vessel wall. Elastin is normally secreted by vSMCs as tropo-elastin, but in very low amounts and the assembly into elastin is limited [342]. Besides the mechanical function provided by elastin, it is also involved in the differentiation of the VSMC themselves (biological functionality). Combining polymer materials with tropo-elastin can therefore enhance the vSMC maturation, and on the other hand also can improve the scaffold's structural properties $[134,343,344]$. More importantly, elastin is normally found in mature vessels whereas a high collagen content is more pronounced in damaged vessel with a synthetic vSMCs phenotype [148, 345]. An elastin-rich matrix would therefore represent a mature blood vessel better than a construct with abundant collagen. This brings us back to the question which differentiation status (and level of functionality) of a construct would be favorable for neovessel formation after implantation. This question is not only applicable to the field of vascular TE but can also be applied to the research question we posed in chapter 5 , involving how the differentiation status of a pre-vascularized implant would influence the bone formation after in vivo implantation. 


\section{Interconnectivity of macro to micro-vasculature}

Screening for the influence of the length of in vitro osteogenic pre-differentiation on vascularization and bone formation in vivo (chapter 5) was important for the eventual implantation in the AV-loop model (appendix 1). Here, it would be favorable to have an already pre-developed capillary bed in an osteogenic tissue, which can interconnect quickly to the sprouting vessels of the autologous AV-loop once implanted. Future studies could also focus on the conversion of the autologous vessel to a (bio)engineered substitute, omitting the problem of donor site morbidity or shortage of donor material. For instance, research on the use of engineered grafts as an arteriovenous fistula for hemodialysis [56, 346-348] or for the femoral AV-loop model [349, 350] has received considerable interest. This points towards the applicability of (bio)engineered vascular substitutes as an arteriovenous loop. However, facilitating the interconnection of a bioengineered vessel to capillaries in vitro or in vivo, as seen in the AV-loop model, is still a challenge that needs to be faced. The in vivo sprouting from the AV-loop occurs as a reaction to a sudden rise in hemodynamic loading and accounts for the proteolytic effect that enables luminal sprouting [351]. This process is mostly visible in the interposed (venous) graft and venous part, and is less pronounced in the arterial side of the loop due to the thick tunica media that prevents the sprouting $[352,353]$. In this thesis, the vascular scaffold in chapter 2 was specifically developed to form a relatively thick tunica media (MEW layer), and to inhibit intimal hyperplasia (dense SES layer). This would counteract the connection of capillaries from the surrounding tissue with the luminal endothelium (appendix 2). To eventually accommodate the interconnection, an altered scaffold design with larger pores to facilitate sprouting would be needed (appendix 2).

Moreover, one could argue that connecting a small diameter vascular graft directly to capillaries is not following the native situation as seen in the vascular tree. Here, the macrovessels first branch off into mesovessels, which then further progress into capillaries [341]. Connecting macrovessels to capillaries would not follow this hierarchy and would disobey Murray's Law; a principle that relates the diameter of hierarchical branches to the parent branch to minimize the required energy for blood transport [354]. However, transmural interconnection of capillaries to a macrovessel is a process that already occurs in the native environment, for example in the provision of blood to the tunica adventitia (internal arising vaso vasorum) [355, 356]. Moreover, transmural ingrowth of capillaries is an event known to take place in vascular graft healing and accounts for one of the ways for endothelialization of vascular conduits [111, 357]. Additionally, in the AV-loop model direct luminal sprouting of capillaries from the autologous vessel occurs, which then later remodel according to the hierarchy of the vascular tree guided by hemodynamic forces (appendix 2). These observations therefore indicate that by provision of the right hemodynamic cues, either in vitro or in vivo, an interconnection segment composed of mesovessels between the two levels of vasculature can be remodeled to accomplish a 
hierarchical network. Thus far, the formation of a complete free standing hierarchical vascular network has not been subject to research, but would be valuable for the construction of a fully functional tissue analog, especially when combined with a, thus far lacking, venous drainage system.

\section{Exploring construct functionality in in vivo situations}

To truly confirm functionality of a bioengineered construct, it should be tested in an in vivo situation. Despite the advances in in vitro complex 3D tissue modelling, in vivo experiments are still indispensable in recapitulating the relevant processes and environment of the human body. As yet, for the development and translation of products towards human clinical studies, devices and materials are obliged to undergo animal testing (FDA, EMA) [358]. In this thesis, the in vivo implantation of the heterotypic vessel was deferred with regards to the insufficient compliance of the current PCL scaffold. Even though vascular scaffolds made from PCL have been implanted, showed relatively good regeneration and provided important insight in their biodegradability [334, 359], implanting a scaffold with insufficient mechanical properties would give profound differences in the outcome parameters compared to those with correct properties. It is known that selecting grafts with a compliance mismatch to the adjacent vessels can induce hyperplasia at the site of anastomosis due to altered shear stress profiles. This would result in an increased incidence of thrombosis and graft occlusion $[60,339,360]$ and could therefore potentially lead to skewed results.

With regards to the animal model used for the bone tissue formation, an immunocompromised rat was selected in which multiple constructs could be ectopically implanted at subcutaneous locations. Using this model, we were able to screen for more conditions with less animals while still being able to review the influence of the complex in vivo situation on bone formation (chapter 5). However, implantation in an orthotopic location would give more insight in the response in a clinically relevant location for bone replacement, for example in a mandibular defect [276], cranial defect [272] or a femur defect [361]. Additionally, although the immuno-incompetent rat model avoids a potentially unfavorable immune reaction to the xenogeneic implant [362], the involvement of the immune response towards implanted materials is highly relevant and can even be favorable for their regeneration properties. To be more precise, the manner in which the host responds to the implanted construct will dictate long-term success or failure. Therefore, use of an animal model closer to the clinical situation would give better insight in the remodeling events, e.g., non-immunocompromised rats and larger models such as goats or sheep.

Besides determining the clinical outcomes of an implanted construct, the immune system can also be actively influenced to steer the regeneration of the construct, for 
example by directing the immune response towards a pro-regenerative phenotype. This can be realized by adaptations of scaffold materials, pore sizes, biofunctionalization with cytokines, scaffold coatings or steering the macrophage polarization with surface topographies [363-365]. Generally, an acute immune response is initiated after biomaterial implantation, characterized by the infiltration of innate cells (i.e., neutrophils and monocytes). The monocytes will become active and polarize into macrophages; the reaction that then will follow depends on the phenotype of polarization and determines if the reaction is driven towards a state of regeneration or towards a chronic inflammation. If the acute response persist into a chronic inflammation, it can culminate into a foreign body response (FBR) on the material's interface, often resulting in failure of the implant [366]. By tuning the initial influx of the innate cells into the implanted construct, the immune response might possibly dampen the transition towards a chronic inflammation. This matter was further build on in chapter 5, where the pre-maturation of the ECM, before implantation, seemed to shield the osteogenic construct from the surrounding tissue and invading macrophages.

The same principle holds for the implantation of vascular substitutes. Often, the initial reaction after the blood-material interaction is the deposition of fibrin to the material's surface via the coagulation cascade. This coincides with an influx of neutrophils and monocytes from the bloodstream and invasion of resident macrophages from the surrounding tissue [364, 365,367-370]. For example, heparin coating of the luminal layer can counteract the coagulation cascade and decrease the leukocyte binding, thereby altering the foreign body reaction [371]. On the other hand, instead of a biomaterial coating, the presence of a functional endothelium could also regulate the initial influx of innate cells. The endothelium is a natural barrier that maintains the anti-thrombotic interface of the scaffold and serves as an immunoregulatory layer that prevents unregulated leukocyte invasion and platelet deposition. Including a functional endothelium, as we did in our bioengineered vascular grafts, might therefore be advantageous for the regulation of the initial influx of the innate cells. Moreover, it would provide an anti-thrombotic layer. This relates back to the benefit of having readily functional tissues or cell layers upon implantation, and favors the development of in vitro pre-cultured tissues as demonstrated in our approach in chapter 2 and 3 over the in situ approach, for this specific matter.

\section{Clinical translation}

Tissue engineering has raised high hopes and expectations about its potential for therapeutic purposes since it was first mentioned in 1993 [372]. Despite the increased interest in $\mathrm{TE}$, only very few discoveries have made it to a clinical product [373]. Concerning this matter, TE faces complex regulatory standards with regards to safety and manufacturing, with products often allocated to more than one regulatory category of the FDA (tissues, biological products, drugs or medical devices) [358]. For successful 
clinical translation of TE products, relevant materials and pre-clinical models are needed with a clear focus on a therapeutic or operative application. Thus far, most research has not focused enough on the clinical applicability of their product or components from the start, which ultimately counteracts the transition of the experimental phase towards a clinical setting.

Herein, the in vitro pre-cultured approach, as investigated in this thesis, and the in situ engineering approach as described elsewhere, both have pros and cons with regards to their applicability. The pre-seeding approach can gain a faster (bio)functionality after implantation, even in tissues with low intrinsic regenerative capacities, whereas the in situ approach enables an off-the-shelf product and omits the use of long and costly pre-culture times [4]. This would make the in situ approach more straight forward to be approved than approaches reliant on living tissues. However, the success of the in situ methodology is also dependent on the regenerative capacity of the patient [372], where difficulties are anticipated with the pathophysiological microenvironment in elderly or diseased patients (e.g., diabetic patients). Overall, different applications may require different approaches, which means that there is not just one uniformly applicable ideal bioengineered product. In both approaches, hurdles need to be taken when translating this to the clinic, also with regard to the work presented in this thesis.

At first, the choice of scaffold material can affect the regulatory process of clinical translation. The material should be gradually biodegradable, allowing the tissue to fully mature over time before the mechanical support of the scaffold is gone, and avoid persistent presence of a foreign body material. Inherent is that the degradation products are not toxic and are not provoking an inflammatory phenotype that could adversely affect remodeling. Both the $\mathrm{PCL}$ and $\mathrm{CaP}$ bioceramic materials presented in this thesis are medical grade and approved for safe clinical applications, and already applied in humans. Contrary to the PCL and CaP ceramics, the hydrogel material Matrigel used in chapter 4 is not of medical grade. Even though Matrigel might appear as an ideal biomaterial, it is unsuitable for clinical application due to high variability in composition, reproducibility and its origin from a mouse tumor [294]. The translation towards the semi-synthetic gelPEG system in chapter 5 , where the low endotoxin gelatin can be crosslinked to the PEG with an FDA-approved Factor XIII component (Cluvot), already makes a step towards the clinical perspective. But, while PEG is a synthetic polymer that is already on the market for medicinal products, its conjugated derivatives contain specific RGD sites (chapter 5 and 6), which have not yet been tested for clinical use. Furthermore, one major challenge for clinical translation is to comply with the high set safety guidelines for the use of a cellbased product [372]. Throughout this thesis, we used clinically relevant cell types, such as MSCs and endothelial colony forming cells (ECFCs), which in principal can be isolated from the patient themselves, to ease a possible transition. 
Ultimately, incorporating all these critical components and overcoming their hurdles to reach for the 'ideal tissue engineered substitute' seems a distant prospect. Therefore, in this journey it is unavoidable to make a trade-off between perfect personalization to the patient, biological function and applicability for production. It is important to, already from the design of translational studies, work according to regulatory guidelines with a close involvement of regulatory bodies. Moreover, there is an elegance in keeping the design and additions as simple as possible. To distinguish the most important necessities for such a tissue-engineered product to be functional, fundamental research that provides knowledge on the in vitro and in vivo biological interactions towards the developed product remains essential. The essence of this knowledge is, having it, to apply it.

\section{General conclusion}

In this thesis, we provided insight in how the scaffold design of a vascular graft can instruct a physiological tissue-like organization and differentiation, and how the functionality of such a macrovessel can be developed by using a bioreactor system to resemble a more physiologically relevant environment. These results highlight the important effects of the structural properties of a scaffold on the biological function. This underlines the link between mechanical functionality and biological function. Moreover, we demonstrated that the tight coupling of angiogenesis and osteogenesis in bone formation can be recapitulated in vitro. To follow up, this principle was used for the pre-vascularization of osteogenically differentiated hydrogel constructs with a capillary network in combination with $\mathrm{CaP}$ materials. We obtained new insights in how the level of pre-differentiation of these hydrogels can influence bone formation in an in vivo setting, with as ultimate goal the implementation in an AV-loop model. These findings are laying the basis for a perfusable hierarchical vascular supply, and once combined, can contribute to the bioengineering of pre-vascularized osteogenic tissues that are readily perfusable upon implantation (Figure 7.1). 




Figure 7.1. Translation of the arteriovenous loop towards clinical applications for the reconstruction of mandibular defects. The arteriovenous loop can be used for the direct anastomosis to the patient's blood circulation. The surrounding matrix can be pre-vascularized in vitro by using a co-culture model (blue capillaries) and would need to interconnect with the sprouts from the arteriovenous loop (intrinsic vascularization, red capillaries) and the surrounding tissue (extrinsic vascularization, green capillaries). This set-up would contribute to the engineering of pre-vascularized osteogenic tissues with a hierarchical vascular supply, that are readily perfusable upon implantation. 


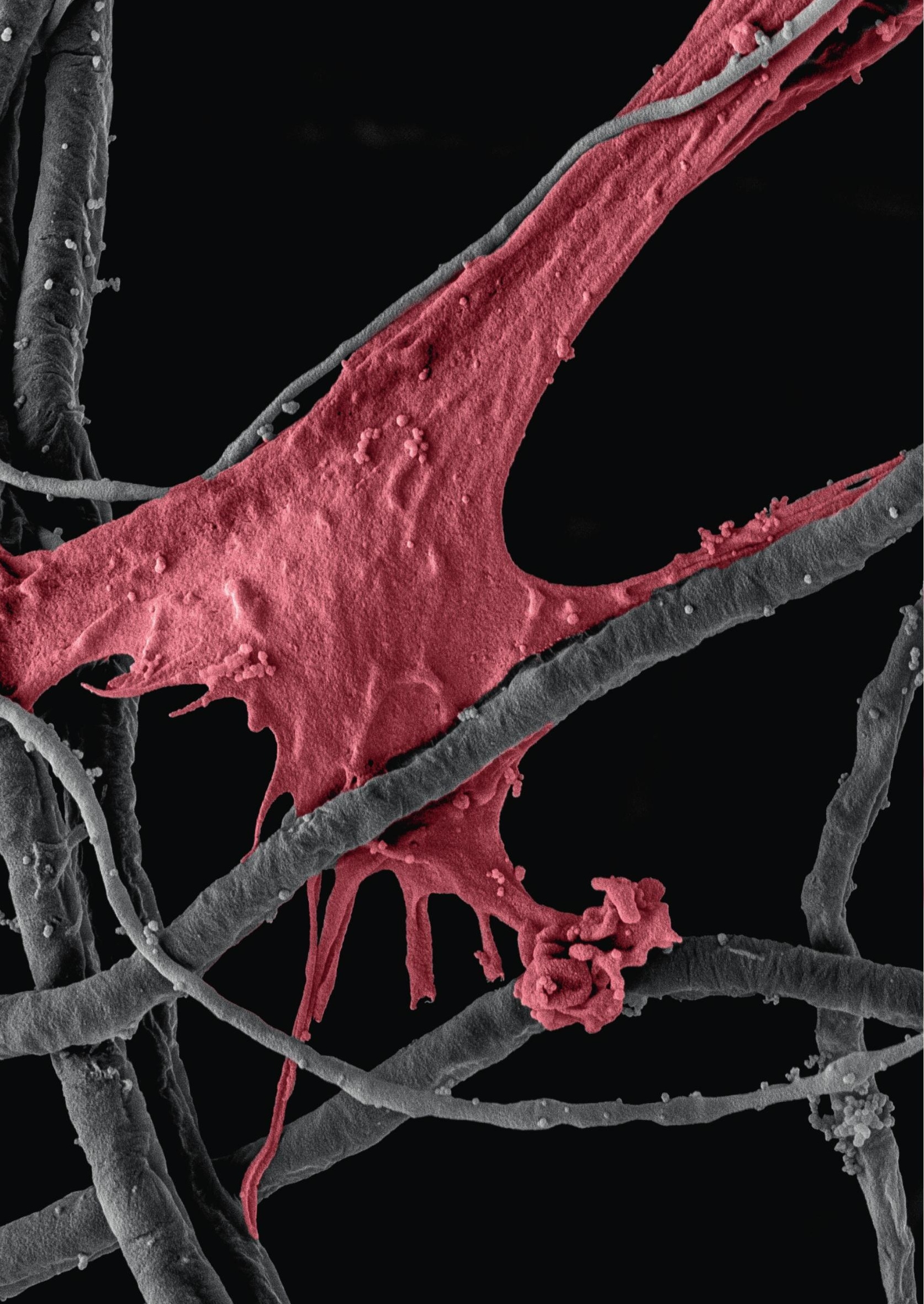




\section{Appendix 1}

Arteriovenous loop model for vascularization of bone tissues

Background information and a study design of the envisioned in vivo follow-up study 


\section{Reconstruction of a critical-size mandibular defect}

The reconstruction of a critical-size mandibular defect is a complex procedure as success largely depends on the restoration of form and function. The current treatment of choice for the reconstruction of critical sized (>6 centimeter) defects is the transplantation of autologous vascularized bone flaps (e.g., radius, iliac crest, fibula flaps) [374, 375]. Vascularized bone flaps are bone grafts taken from a donor site with the intact blood supply, surrounding muscle and skin still attached, and are used to replace the damaged mandible (Figure 1). The surgical procedure, from e.g., the fibula as donor site, provides a vascularized bone graft up to $25 \mathrm{~cm}$ of bone which can be shaped to fit mandibular implantation via osteotomy. The graft can be directly connected to the blood circulation by means of surgical anastomosis [374, 376, 377] (Figure 1). However, the procedure has several disadvantages, including donor site morbidity $(35 \%$ of the cases $[376,378])$ and infections ( $10 \%$ after one week). Additionally, problems with size mismatch after transplantation were reported, where 1 year after surgery $22 \%$ of the patients rate their facial contours as only adequate (15\%) or poor (7\%) [375]. Additionally, $17 \%$ of the cases demonstrated failure of the bone graft after one year after surgery, underlining the need for alternative approaches [375].
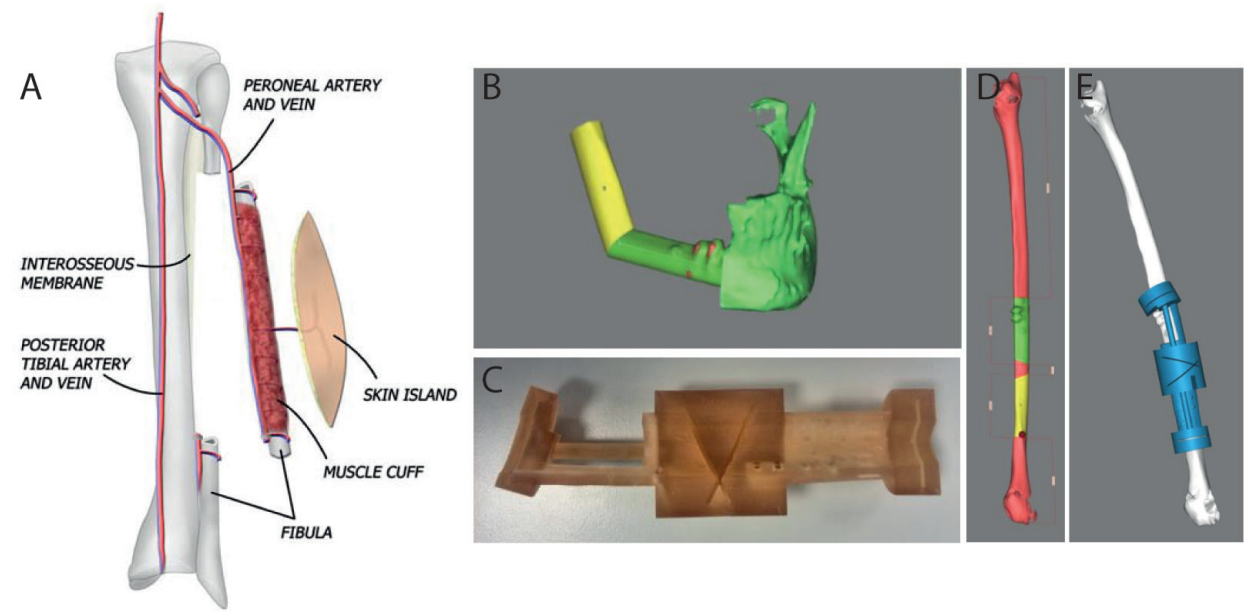

Figure 1. Schematic presentation of the vascularized fibula graft. A) The fibula is dissected with the intact muscles and skin island attached. The flap will then be transplanted to the defect site. Reproduced from Dr. R. Buntic with permission, online atlas of microsurgery. B) The surgery is planned pre-operatively with 3D reconstruction software in the 3D facelab of the University Medical Center Utrecht (UMCU), personalized to the patient. C) A personalized saw-guide is made which is placed on the fibula bone, guiding the surgeon where to saw the bone. D) The fibula is shaped to fit the mandibular defect according to the pre-operative planning and E) saw according to this. Images reproduced from the 3D facelab UMC Utrecht. 


\section{Arteriovenous-loop model for the vascularization of tissue constructs}

A promising alternative is the arteriovenous loop (AV-loop) model. In this model, an artery is surgically anastomosed to a vein in the shape of a loop, with an interpositional venous graft (IVG) to achieve sufficient loop length (Figure 2) [379]. The AV-loop model serves a dual role; it is an in vivo model that can be translated for the vascularization of engineered constructs for clinical practice. On the other hand, it also can represents a closed isolated system for in vivo screening purposes for matrices, cells and growth factors. The vascularization of tissue constructs is realized by enveloping the axial vessel with a tissue chamber, which can be filled up with e.g., hydrogels, cells or in case of bone tissue engineering calcium phosphates (CaPs). Capillary sprouting will occur from the vascular axis into the surrounding tissue, most likely induced by hypoxia [380-382] and hemodynamic changes [383]. By using a solid 'closed' chamber wall, a controlled environment for the implanted cells, growth factors and matrices is created, and vascularization will solely occur from the axial vessel, defined as intrinsic vascularization (Figure 2). In case where an open porous chamber is used, blood vessels may also sprout from the surrounding matrix into the tissue chamber (extrinsic vascularization), which is promoting a faster vascularization of the full tissue [91, 93, 95] (Figure 2). Since the development of the AVloop model in 1980 by Erol and Spira [384], researchers have implemented various types of scaffolds in the chamber, e.g., poly(lactic-co-glycolic acid) (PLGA) $[385,386]$ or hydrogels such as fibrin [90, 353, 386], Matrigel [386] or collagen type I [351, 387, 388]. Moreover, the model has been exploited for the vascularization of various tissue types e.g., skin [351, 388], liver [389], heart muscle [390], skeletal muscle [391] and lymphatic tissue [392].

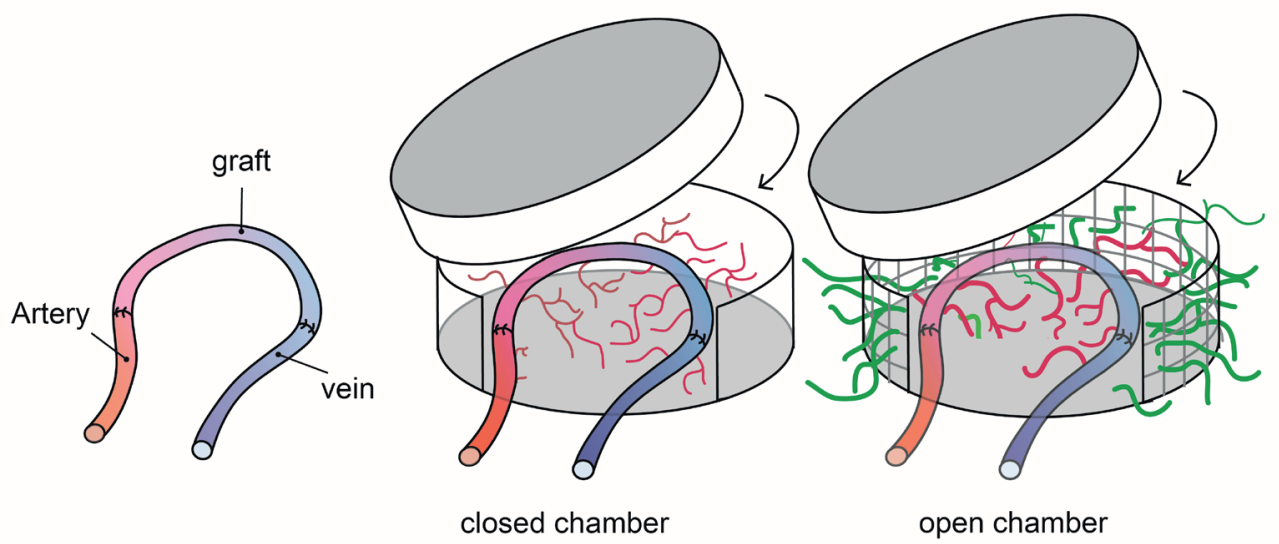

Figure 2. Arteriovenous loop model. An arteriovenous (AV)-loop can be made by anastomosing an artery to a vein with an interpositional (venous) graft (IVG). By placing the loop in a closed tissue chamber, solely intrinsic vascularization (red capillaries) will occur. Placing the AV-loop in an open porous chamber also allows vessel from the surrounding tissue to grow in the chamber (extrinsic vascularization, green). 


\subsection{Arteriovenous-loop for bone tissue engineering}

In 2006 the AV-loop model was used for the first time to vascularize bone constructs in the groin of rats. Here, the chamber was supplemented with processed bovine cancellous bone (PBCB) matrix [100]. Hereafter similar approaches for bone tissue engineering (BTE) were used, for example by incorporating hydroxyapatite (HA)/ $\beta$-tricalciumphosphate granules ( $\beta$-TCP) [90, 93-95, 98, 276, 393-395], 100\% $\beta$-TCP [396], coral bone [397] or NanoBone [91] with or without growth factors (BMP2, VEGF, FGF) $[92,98,276,393]$ or cells (osteoblasts, MSCs) [92, 93, 393]. These approaches contributed to the creation of bone tissues in rat (groin, ectopic and femoral defect, orthotopic), sheep (groin, ectopic), rabbit (groin, ectopic), dogs (groin, ectopic) and goat (mandible, orthotopic) models. Recently, the model was successfully implemented in a patient to reconstruct a radial bone defect, but only for a relatively small defect [102]. Moreover, a custom made vascular bone graft has already been used to repair a mandibular discontinuity in human patients, but required the use of high concentrations of BMP7 and a pre-implantation period in the latissimus dorsi muscle for 7 weeks [398]. Currently, researchers are working towards the first clinical trials using the AV-loop model in mandible reconstruction after segmental resection $[97,276]$.

Nevertheless, in most of the animal studies presented above, a lack of bone formation was frequently observed in the constructs implanted in rat $[90,92,95,100,395]$, dog [396] or sheep [90, 394, 399]. In cases where bone formation did occur, the determining factors appeared to be the addition of the growth factor BMP2 [93, 276, 393, 394], with or without osteogenically stimulated MSCs or osteoblasts [93, 393, 394]; or by making use of the clinically approved NanoBone as matrix in a large animal model [91]. This indicates that the addition of cells or growth factors to the matrix is necessary for de novo bone formation. The dependence of de novo bone formation on the inclusion of osteogenically stimulated donor cells was also confirmed in our own study (chapter 5). Here, the presence of differentiated cells in the implant was a crucial trigger for the initiation of bone formation in an ectopic rat model, suggesting that this would also be required for the initiation of bone formation in the AV-loop model.

However, without immediate blood perfusion, problems with the inclusion of cells in the chamber will arise with cell death as a consequence [94]. Although the blood vessels eventually sprout towards the cells in the construct after it is implanted in vivo, it takes 2 weeks to fully vascularize the chamber around the AV-loop [95, 353, 379, 381]. This timeframe is not sufficient to supply blood to the core tissue before it turns necrotic [280]. Some groups therefore chose to only include the cells in the chamber after a 2 week in vivo matrix pre-vascularization [94, 400]. We suggest that a cell-based engineered tissue construct with an in vitro engineered intrinsic capillary network would be beneficial for the implantation in the AV-loop model. This would enable a faster connection of the 
engineered capillary bed to the autologous AV-loop, rather than solely relying on the sprouts arising from the AV-loop itself. In this chapter we propose a study design that would facilitate the formation of such constructs.

\section{Experimental set-up for the envisioned in vivo arteriovenous-loop study}

The MSC-ECFC hydrogel co-culture model, as described in chapter 4 and 5, will serve as a basis for the pre-vascularization of the matrix to be included in the tissue chamber (Figure 3). In this model, a pre-vascular network was developed already after 5 days, with adjoining a-smooth actin (a-SMA) structures that support the vascular bed. Ideally, the construct created for the inclusion in the AV-loop model is, besides pre-vascularized, also composed of a robust and porous matrix that is replaced by bone tissue over time. Therefore we selected an $35 / 65 \% \mathrm{HA} / \beta$-TCP material with submicron topography, combined this with the MSC-ECFC co-culture model (chapter 5) and osteogenically pre-differentiated them in vitro. In chapter 5 we screened for the optimal pre-culture time (6 or 14 days) of the hydrogel construct for the future application in the AV-loop model. Here, both pre-culture times proved to be suitable in the initiation of de novo bone formation. However, the 14 day pre-culture period triggered an accelerated bone formation upon implantation and resulted in more human cell retainment in the construct.

This now opens the discussion of what the most favorable situation would be for implantation in the AV-loop model. On one hand we have the 6 day pre-cultured construct in which the matrix induces the host cells to participate in the bone forming process, resulting in bone derived from both the donor and host cells. On the other hand we have a 14 day pre-cultured where faster initiation of bone formation was observed, but with the bone solely formed by the implanted host cells after 8 weeks. Either way, promotion of the endogenous regeneration by the implanted TE material resulting in a self-sustaining bone construct would be the best option in our opinion. Therefore we postulate that implanting the 6 day pre-culture period would be the most favorable option. Additionally, the shorter pre-culture period would also result in less influences of the CaP granules on the vessel network as we currently encounter (shown in chapters 5 and 6).

To summarize the set-up, we envision combining the $35 / 65 \% \mathrm{HA} / \beta-\mathrm{TCP}$ CaP granules with the human MSC-ECFC co-culture model in the gelPEG hydrogel system, and pre-culture it for 6 days. Subsequently, we will implant it in the AV-loop model in an immunocompromised rat ( $\left.\mathrm{Crl}: \mathrm{NIH}-F o x n 1^{\mathrm{rnu}}\right)$. The chamber will be implanted at an ectopic location in the groin. Here, the femoral artery will be anastomosed to the femoral vein with an IVG $(\sim 1.5 \mathrm{~cm})$ from the contralateral side, and will be enveloped by a porous $3 \mathrm{D}$ printed poly( $\varepsilon$-caprolactone) $(\mathrm{PCL})$ chamber (Figure 3$)$. Constructs will be implanted for up to 12 weeks and, so far, the main outcomes will be based on vascularization on 
both early and late time-points (Microfil and immunohistochemistry) and de novo bone formation ( $\mu \mathrm{CT}$, histomorphometry). Since the AV-loop surgery is complex and requires sophisticated microsurgical skills, a pilot phase was performed in we confirmed the feasibility of the microsurgery approach. In the next phase, vessel patency and thrombus formation will be studied in the anastomosed graft over a relatively short time by using a Doppler system. Here, the vessel diameter, flow speed and turbulence will be monitored to confirm the success rate of the surgery and to provide base-line data. Hereafter, the study will be continued by the addition of the tissue chamber with the CaP co-cultured hydrogel. Future studies will be aimed at translation to bigger animal models (e.g., goat) with orthotopic implantation (e.g., mandibular defect) with the final aim of translating it towards clinical applications for the reconstruction of mandibular defects.

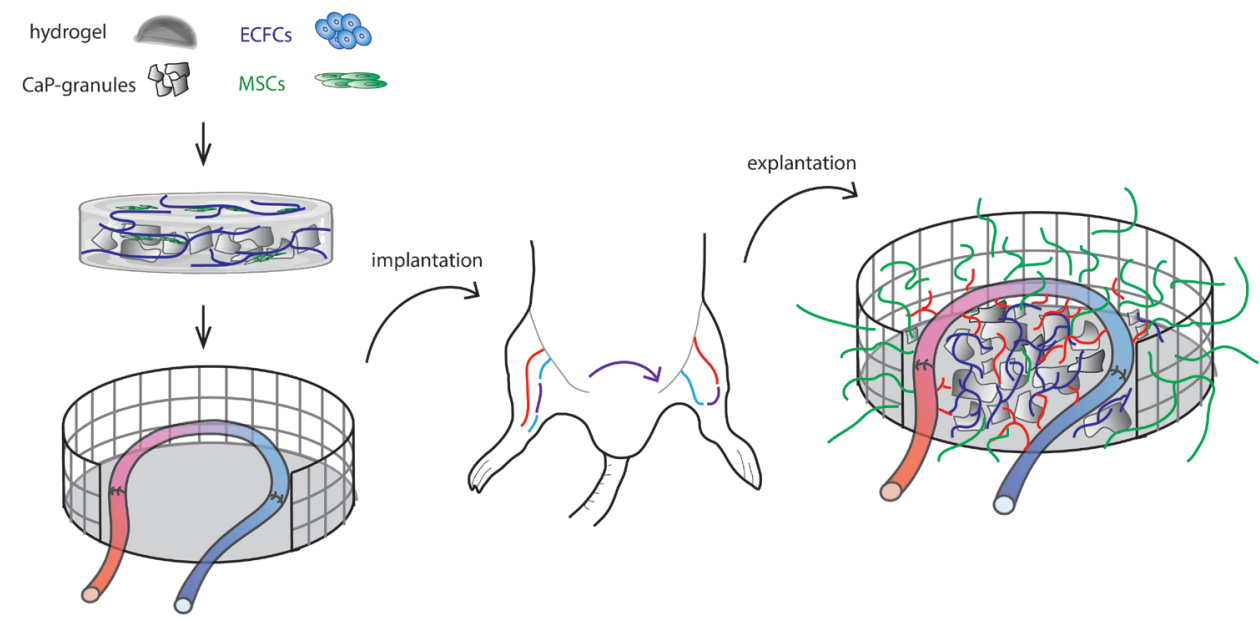

Figure 3. Experimental set-up arteriovenous loop model. Multipotent mesenchymal stromal cells (MSCs) and endothelial colony forming cells (ECFCs) will be mixed in a hydrogel together with calcium phosphate (CaP) granules for in vitro pre-vascularization; this will serve as the matrix in the tissue chamber. The AV-loop with matrix will be placed in an open porous chamber and implanted in the groin. This will allow for intrinsic vascularization (red capillaries) and extrinsic vascularization (green capillaries) to grow into the construct and to connect to the in vitro developed prevascularized tissue (blue capillaries). 


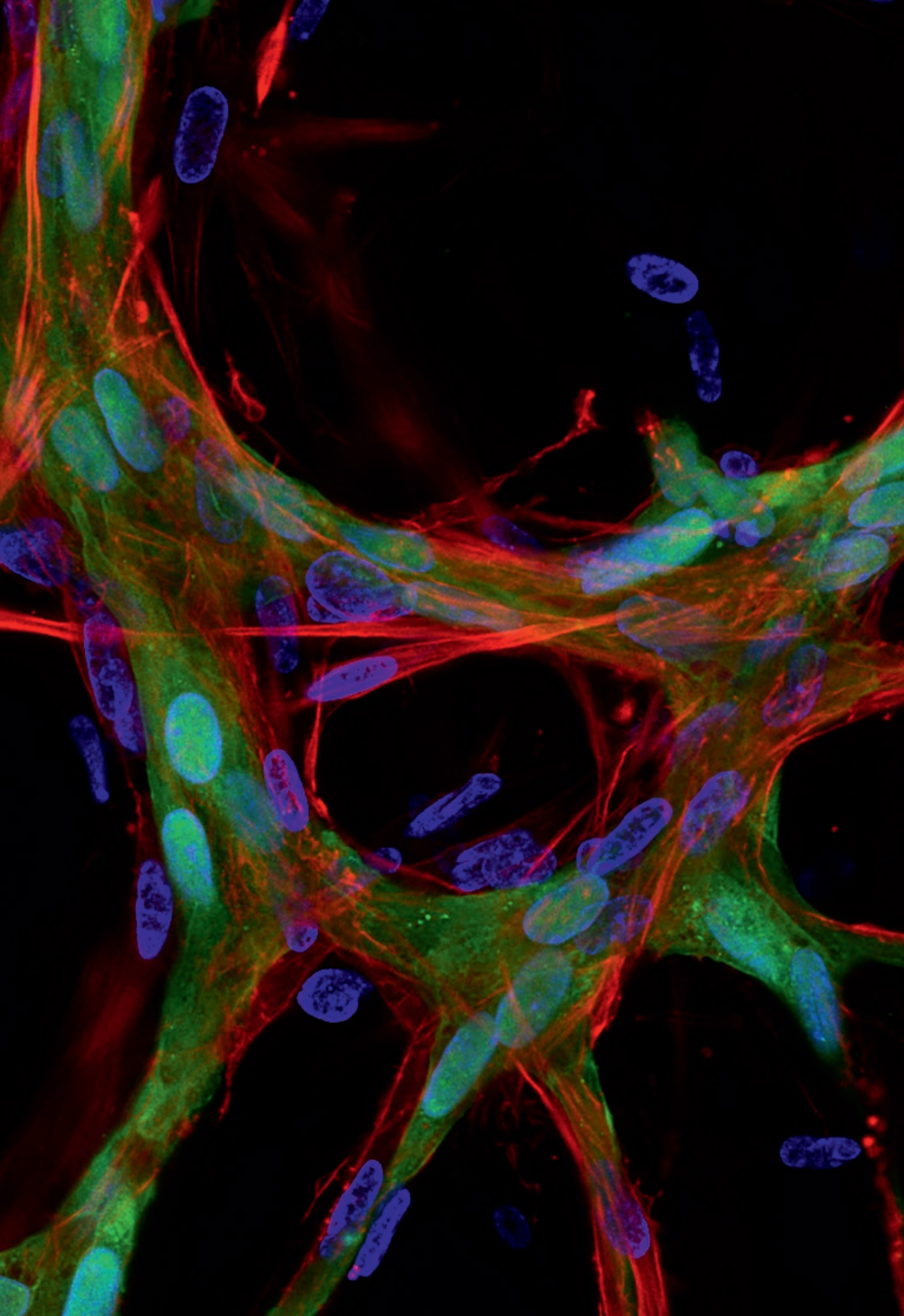




\section{Appendix 2 \\ In vitro interconnectivity of multi-level vasculature}

Background information and the envisioned experimental

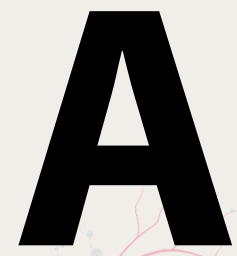
set-up 


\section{Translating the native vascular tree to a multi-scale vascular network in vitro}

Recapitulating the hierarchical network of blood vessels in vitro as seen in the native body might be the key for successful implantation of large engineered tissues. This study proposes a novel system for the engineering of multi-scale vascularized tissue by molding a co-culture hydrogel (chapters 4 and 5) around the developed bi-layered vascular graft (chapters 2 and 3). When seeding both components with a co-culture of multipotent mesenchymal stromal cells (MSCs) and endothelial colony forming cells (ECFCs), this system would potentially allow for the sprouting of capillaries out of the vascular graft into the vascularized hydrogel layer. By aiming for the capillaries to be connected to the vascular graft, this design would realize a direct perfusion of an entire (osteogenic) construct after implantation. To allow this, it is suggested that the vessels have to be organized as a vascular tree. This means that the macrovessel (artery or vein, $>100 \mu \mathrm{m}$ $\varnothing$ ) is branching out into smaller vessels (mesovessels, $10-100 \mu \mathrm{m} \varnothing)$ and eventually into capillary sized vessels $(<10 \mu \mathrm{m} \varnothing)$, mimicking the natural design of a vascular network [33-35].

This principle has already been used in the arteriovenous (AV)-loop model, where an autologous vessel is used as an axial vascular access from which capillaries sprout into the surrounding material or tissue in a chamber (appendix 1). Future prospects are on the advancement of the autologous vessel to an engineered substitute, omitting the problem of donor site morbidity or shortage of donor material. An engineered AV-fistula for hemodialysis patients has already proven its applicability [56, 346-348] and indicates the possible use of a bioengineered vessel in an AV-loop model for the vascularization of tissues. However, facilitating the interconnection of a bioengineered vessel to capillaries in vitro or in vivo, as seen in the AV-loop model, is still a challenge that needs to be faced. There have already been successes in creating capillary beds, small diameter blood vessels and small arteries $(0.5 \mathrm{~mm}-1 \mathrm{~mm})$ individually, and in few cases combinations of both meso- and microvasculature (reviewed in [48]). But, there has been no success in achieving a multi-scale dynamic blood vessel network combining all the levels of vasculature in one construct, that would be applicable for implantation [341].

\section{Formation of a hierarchical vessel network in the native situation}

When mimicking a hierarchical vasculature in vitro, it is important to know how this evolves in the natural in vivo situation. During embryogenesis, the nascent vascular network is formed through vasculogenesis (de novo blood vessel formation) and angiogenesis (sprouting of new vessel from preexisting ones), which thereafter matures, recruits mural cells and remodels into a hierarchical network [401]. How the vascular network with the vessels and sprouting structures exactly evolves into a hierarchical structure is not yet fully understood, but it is likely to be driven by epigenetic factors and hemodynamic forces 
that control the remodeling into the shape of a vascular tree [341, 401, 402]. Indeed, in the mammalian body the blood vessel structures and diameter are found to be highly related to blood flow pattern to ensure a proper function, which is in agreement with Murray's Law [341]. Here, the diameter of the daughter branch is related to the diameter of the parent branch, such that the energy for pumping the blood is minimized [354].

Recently it was hypothesized that a shear force above a certain threshold can trigger circumferential growth, meaning that the diameter of the vessel enlarges to ensure a lower shear stress to prevent endothelial dysfunction [354]. This indicates that hemodynamic forces can indeed be the trigger for a newly sprouted vascular structure to form a lumen (tubulogenesis) and remodel into a mesovessel that then diverges into a capillary. The enlargement of a vessel diameter is also a specific phenomenon occurring during the process of arteriogenesis. Here, an increase in hemodynamic forces caused by acute occlusion of a major vessel (e.g., infarct) is the driving force for mural cell recruitment and proliferation. This then drives for a preexisting collateral vessel to remodel into a functional artery with an enlarged diameter $[402,403]$. Taken together, this indicates that physical forces (e.g., altered shear stress) can be the driving factor that supports the recruitment of mural cells, the development of functional vessels and the formation of the vascular tree during embryogenesis [341]. This suggests that by providing the right hemodynamic cues in the experimental set-up, sprouts can arise from a main axial engineered vessel which later on can remodel in mesovessels, establishing the interconnection between the two levels of vasculature.

\section{Development of a multi-scale vascularized tissue construct in vitro}

The proposed research will make a first step towards developing a technique to create a branching vascular structure within an engineered osteogenic tissue by including two different levels of vasculature (i.e., a macrovessel and a capillary bed). The basis of the design will be the bi-layered small diameter vascular graft described in chapters 2 and 3. This graft consists of two scaffold layers created with solution electrospinning (SES) and melt electro writing (MEW), designed to mimic the tunica intima and tunica media, respectively (Figure 1). The fibrous inner layer consists of a dense isotropic network of small fibers $(1-1.5 \mu \mathrm{m})$ and small pores $(<10 \mu \mathrm{m})$. This layer was designed to serve as a substrate onto which the ECs could form a confluent endothelium [110], and to prevent intimal hyperplasia (ingrowth of vascular smooth muscle cells (vSMCs) into the luminal layer), where the latter is one of the problems normally causing occlusion in small diameter vessels. The open porous medial layer consists of fibers with a larger diameter $(15 \mu \mathrm{m})$ that are oriented in a near-circumferential direction, mimicking the native collagen distribution $[58,131,133,332,333]$ and facilitating the formation of orientated multi-layered sheets of vSM-like cells. For the formation of the capillary bed, a hydrogel containing a co-culture that self-assembles into a pre-vascular structure will be used 
(chapter 4 and 5). However, connecting the two levels of vasculature to each other with this vascular graft is challenging.
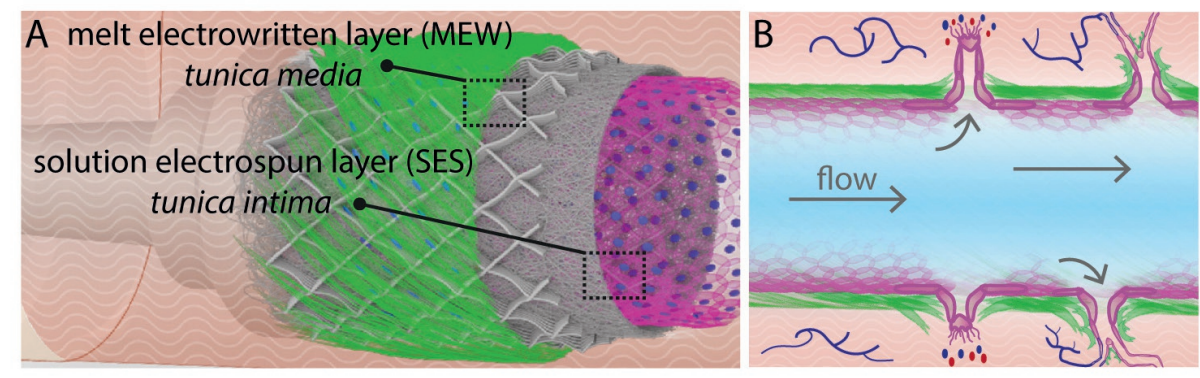

Figure 1. Design of the bi-layered vascular graft. A) The heterotypic scaffold layers were created with solution electrospinning (SES) and melt electro writing (MEW), designed to mimic the tunica intima with a confluent endothelium (purple) and tunica media with multiple layers of vSMCs (green). B) For the capillaries (blue) in the hydrogel to connect to the vascular graft, sprouting capillaries (purple) would need to form through the scaffold and tissue layers (transmural capillarization), either direct by hemodynamic forces (arrows) and/or growth factors signals (blue/red dots). Image adapted from I. Pennings and E. van Haaften et al., 2019.

\subsection{Adapting the scaffold structure to facilitate interconnection}

For the effective connection of the newly formed capillaries to the vascular scaffold, these capillaries have to be able to penetrate through the different scaffold and tissue layers (Figure 1). To enable this, the migration of ECs through the SES layers first has to be possible. The dense fibrous structure of the SES layer is considered a boundary when it comes to allowing cell migration. With electrospinning, the fiber diameter and pore size are inherently connected to each other, meaning the smaller the fiber diameter the smaller the pore [61]. Where MSCs and ECs in general are able to migrate through pores of around $8 \mu \mathrm{m}$ [404], this will not be enough to allow capillaries $(10 \mu \mathrm{m} \varnothing)$ or functional arterioles $(25 \mu \mathrm{m} \varnothing)$ with accompanying mural cells to form though $[36,48,111,357,405]$. These numbers suggest that the pore diameter has to be considerably larger than the size required for cell migration, but on the other hand pores that are too large (>90 $\mu \mathrm{m})$ promote fibrovascular infiltration $[111,406]$.

Ingrowth or outgrowth of capillaries, (i.e., transmural capillarization) in synthetic grafts is already known to be one of the potential sources that enhances spontaneous in vivo endothelialization in vascular conduits [111, 357]. For transmural endothelialization to occur in our bioengineered graft, there should be an adequate scaffold porosity with sufficient pore-interconnectivity throughout the scaffold layer to allow for the ingrowth of perivascular tissues across the complete graft wall. Grafts with a pore size of $25-40 \mu \mathrm{m}$ (microporous 
polyurethanes (PU) grafts) or $<60 \mu \mathrm{m}$ internodal distance (polytetrafluoroethylene (ePTFE) grafts) have been reported to allow the ingrowth and outgrowth of capillaries in synthetic graft after implantation [111, 357, 404, 407, 408]. It is therefore believed that the ideal interconnecting pore size for vascular sprouting lies between $25-40 \mu \mathrm{m}$. Additionally, it would be useful to look into the implementation of interconnected pores with defined sizes on set locations in the SES layer of our bi-layered graft.

In order to maintain the tight SES layer while also having bigger pore sizes at defined locations, and still preserving the mechanical properties of the scaffold, fabrication modification techniques can serve as a solution: e.g., electrospraying, ice crystals, changes in electric field, spike puncturing or laser/ultraviolet irradiation (reviewed in $[48,409]$ ). Another technique that might be applicable for this purpose makes use of laser ablation to micropattern pores in electrospun meshes [410, 411]. Laser ablation is an attractive approach because it is a non-contact one-step procedure without the use of additional chemicals or materials, and can produce a range of pore diameters [410, 411]. Moreover, the laser beams are expected to form a smooth pore surface due to heat-induced melting of the polymer. However, the feasibility of creating pores in our scaffold with these techniques has not been established yet.

\subsection{External factors that induce luminal sprouting through the tunica media}

The migration of capillaries through the tunica media might also cause problems in addition to the migration through the SES layer. The in vivo sprouting angiogenesis from the AV-loop in rats mostly occurs in the interposed venous graft (IVG) and venous part ( $5 \pm 1$ cells thick [412]) thanks to their relatively thin tunica media [352, 353]. The slightly denser vSM-like layer in our engineered graft might therefore compromise the sprouting. However, the proteolytic effect of shear stress activated ECs and metalloproteinases (MMPs) makes it possible for capillaries to penetrate through the VSMC layers of the femoral artery in the AV-loop model, though to a lesser extent, which hints towards the applicability to the bioengineered vessel.

The in vivo direct luminal sprouting from the AV-loop normally occurs as a reaction to a sudden rise in hemodynamic loading and hypoxic factors in the tissue construct [351], related to the embryonic nascent network development as described in section 2 . This is associated to the fact that fluid shear stress regulates angiogenic sprouting and upregulation of MMPs together with vascular endothelial growth factor (VEGF) [341, 413]. Sprouting normally occurs via the increase in concentration of VEGF, which destabilizes and permeabilizes the existing endothelium, allowing fibrin to leach into the endothelial microenvironment which then serves as a temporal scaffold for tubulogenesis and migration [341, 353, 388, 414]. The tip-cell of the structure is leading the sprout into the hypoxic tissue along the chemotaxis of growth factors (VEGF), and is aided via the matrix degradation by the activated MMPs [106, 341]. 
Translating the knowledge of the embryonic development and the AV-loop model to an in vitro set-up, pro-angiogenic growth factors and hemodynamic forces seem to be indispensable for the creation of an interconnected multiscale vascular network. Nguyen et al., already pin-pointed a pool of growth factors that can induce sprouting angiogenesis and promote vessel stabilization in vitro. This mix of growth factors promoted interconnection between sprouts and vascular channels in bulk hydrogels. This mixture was composed of monocyte chemotactic protein-1 (MCP-1), VEGF, phorbol 12-myristate 13-acetate (PMA) and sphingosine-1-phosphate (S1P) (MVPS) [106] and might be of use in our experimental set-up. When focusing on the application of mechanical stimulation, flow perfusion can be applied by dynamically culturing the vessel in the bioreactor system that was developed in chapter 3. Ideally, the flow-induced direct luminal sprouting will contribute to the formation of a hierarchical system, including macrovessels, mesovessels and capillary bed.

Taken together, to eventually accommodate the interconnection in vitro, we would ultimately need to make a trade-off in the scaffold design (i.e., pore fabrication), we would likely need to have an hemodynamic stimulus and provide a growth factor gradient. In the next section, a first step was made by designing a mold for casting of the co-culture hydrogel around the vascular graft, and serves as a basis for the further development of the study that focusses on the actual interconnectivity.

\section{Experimental set-up and preliminary data}

\subsection{Mould design and cell seeding}

In the first step, a negative imprint of the desired mold was created using stereolithography, and hereafter casted with polydimethylsiloxane (PDMS) (Figure $2 \mathrm{~A}-\mathrm{B}$ ). The mold was designed specifically to enable hydrogel molding around the graft when it is attached to the two connection points of the perfusion loop in the bioreactor set-up (Figure 2C). The design allowed for a uniform $400 \mu \mathrm{m}$ thick layer of gelatin PEG (gelPEG, chapter 5 ) to be formed around the bi-layered scaffold (Figure 2D). The specific $400 \mu \mathrm{m}$ thickness of the hydrogel was based on the diffusion limit of $200 \mu \mathrm{m}$ in native tissue, assuming that the gel is in contact with the medium both on the outside and luminal side of the graft.

In the second step, the feasibility of casting a MSC-ECFC encapsulated hydrogel layer around a seeded vascular graft was examined. The hydrogel layer attached well to the graft when the latter one was seeded with multipotent mesenchymal stromal cells (MSCs) on the outside and endothelial colony forming cells (ECFCs) on the inside (Figure $2 \mathrm{E}-\mathrm{F}$ ). Additionally, the MSC-ECFC co-culture self-assembled into vascular structures when cultured in endothelial growth medium (EMG), with a homogenous distribution of the endothelial structures throughout the hydrogel layer (Figure $2 \mathrm{E}-\mathrm{F}$ ). 
This preliminary data show the feasibility of molding a gelatin-based hydrogel around the PCL scaffold, and gives a starting point to further enable the interconnectivity of the two levels of vasculature in the bioreactor system (Figure $2 \mathrm{G}$ ). The next steps will therefore be focused on assessing if i) defined pore sizes can be created in the scaffold and if cells can migrate through it, if ii) supplementing the hydrogel or the (osteogenic/vasculogenic) culture medium with the MVPS growth factors induces sprouting and subsequently interconnection, and finally if iii) the construct can be mounted in the bioreactor system to allow for perfusion of the vascular network.

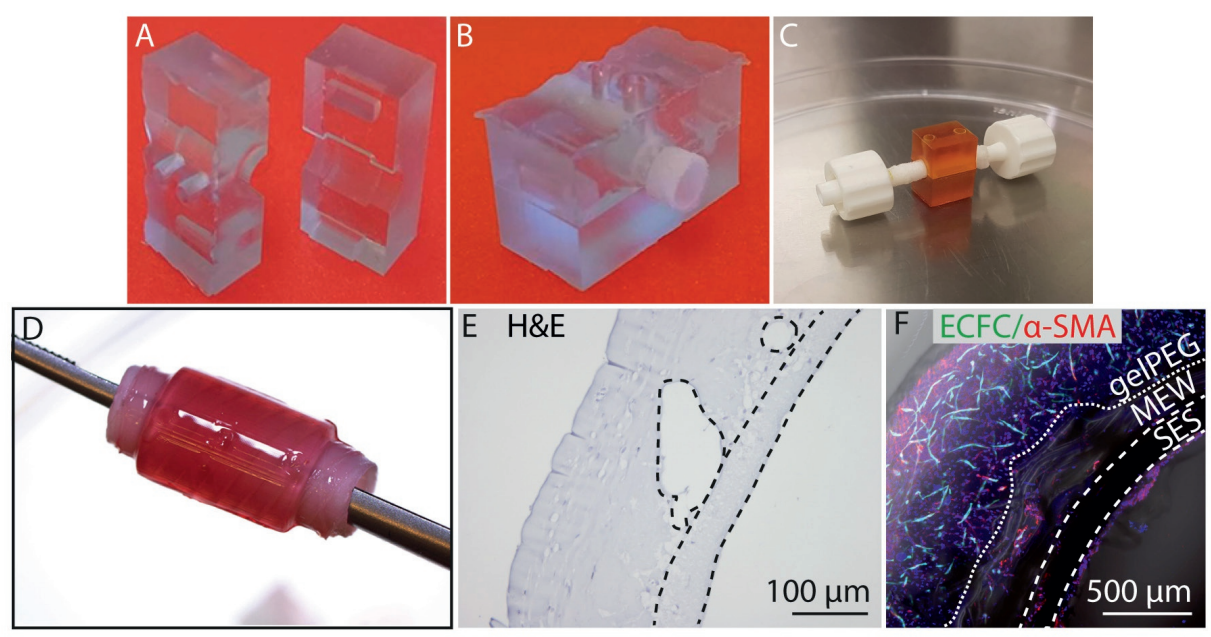

G



Figure 2. Design of the mold for casting of the hydrogel layer around the bi-layered graft. A-B) A PDMS mold was created that enables hydrogel casting around the vascular graft, $C$ ) also when already attached to the perfusion connection points of the bioreactor system. Courtesy of A. van Soest \& A. van Spijk. D) It was shown that a uniform hydrogel layer can be formed around the graft, E) which attaches well to the vascular graft, and shows F) an equal cell distribution and formation of vascular structures in the gel. G) The end-goal is mounting the hydrogel-graft construct in the bioreactor system for the application of a perfusable osteogenic construct. Image adapted from I. Pennings and E. van Haaften et al., 2019. 

References

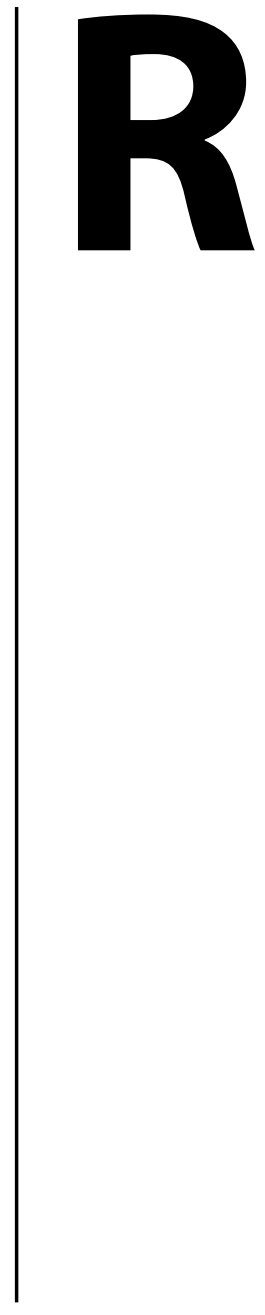




\section{References}

1. Ghosh, S., Human regeneration: An achievable goal or a dream? J Biosci, 2016. 41(1): p. 157-65.

2. Poss, K.D., Advances in understanding tissue regenerative capacity and mechanisms in animals. Nat Rev Genet, 2010. 11(10): p. 710-22.

3. Smith, B.T., et al., Bone Tissue Engineering Challenges in Oral \& Maxillofacial Surgery. Adv Exp Med Biol, 2015. 881: p. 57-78.

4. Mao, A.S. and D.J. Mooney, Regenerative medicine: Current therapies and future directions. Proc Natl Acad Sci U S A, 2015. 112(47): p. 14452-9.

5. Langer, R. and J.P. Vacanti, Tissue engineering. Science, 1993. 260(5110): p. 920-6.

6. Furth, M.E. and A. Atala, Tissue Engineering, in Principles of Tissue Engineering. 2014. p. 83-123.

7. Amini, A.R., C.T. Laurencin, and S.P. Nukavarapu, Bone tissue engineering: recent advances and challenges. Crit Rev Biomed Eng, 2012. 40(5): p. 363-408.

8. Kanczler, J.M. and R.O. Oreffo, Osteogenesis and angiogenesis: the potential for engineering bone. Eur Cell Mater, 2008. 15: p. 100-14.

9. Futran, N.D. and E. Mendez, Developments in reconstruction of midface and maxilla. The Lancet Oncology, 2006. 7(3): p. 249-258.

10. Hall, B.K., Bones and Cartilage : Developmental and Evolutionary Skeletal Biology. 2015, San Diego, United States: Elsevier Science \& Technology.

11. Buckwalter, J.A., et al., Bone biology. II: Formation, form, modeling, remodeling, and regulation of cell function. Instr Course Lect, 1996. 45: p. 387-99.

12. Buckwalter, J.A., et al., Bone biology. I: Structure, blood supply, cells, matrix, and mineralization. Instr Course Lect, 1996. 45: p. 371-86.

13. Lerner, U.H., Bone remodeling in post-menopausal osteoporosis. J Dent Res, 2006. 85(7): p. 584-95.

14. Zhou, H., S.L. Lu, and W.D. Dempster, Bone Remodeling: Cellular Activities in Bone, in Osteoporosis in Men (Second Edition), J.P.B. Eric S. Orwoll, Dirk Vanderschueren, Editor. 2010, Academic Press p. $15-23$.

15. Marsell, R. and T.A. Einhorn, The biology of fracture healing. Injury, 2011. 42(6): p. 551-5.

16. Boskey, A.L., The Biochemistry of Bone: Composition and Organization, in Osteoporosis in Men (Second Edition), J.P.B. Eric S. Orwoll, Dirk Vanderschueren, Editor. 2010, Academic Press p. 3 - 13.

17. Proff, P. and P. Romer, The molecular mechanism behind bone remodelling: a review. Clin Oral Investig, 2009. 13(4): p. 355-62.

18. Mao, J.J., et al., Craniofacial tissue engineering by stem cells. J Dent Res, 2006. 85(11): p. 966-79.

19. Hidalgo, D.A., Fibula free flap: a new method of mandible reconstruction. Plast Reconstr Surg, 1989. 84(1): p. 71-9.

20. Taylor, G.I., P. Townsend, and R. Corlett, Superiority of the deep circumflex iliac vessels as the supply for free groin flaps. Clinical work. Plast Reconstr Surg, 1979. 64(6): p. 745-59.

21. Bae, D.S., P.M. Waters, and M.C. Gebhardt, Results of free vascularized fibula grafting for allograft nonunion after limb salvage surgery for malignant bone tumors. J Pediatr Orthop, 2006. 26(6): p. 809-14. 
22. Alaribe, F.N., S.L. Manoto, and S.C.K.M. Motaung, Scaffolds from biomaterials: advantages and limitations in bone and tissue engineering. Biologia, 2016. 71(4).

23. Mobini S, A.A., Bone Tissue Engineering in the Maxillofacial Region: The State-of-the-Art Practice and Future Prospects. Regen Reconstr Restor. 2016, 2016. 1(1): p. 8-14.

24. Bohner, M., L. Galea, and N. Doebelin, Calcium phosphate bone graft substitutes: Failures and hopes. Journal of the European Ceramic Society, 2012. 32(11): p. 2663-2671.

25. Mishra, R., et al., The potential impact of bone tissue engineering in the clinic. Regen Med, 2016. 11(6): p. 571-87.

26. Temple, H.T. and T.I. Malinin, Orthobiologics in the Foot and Ankle. Foot Ankle Clin, 2016. 21 (4): p. 809-823.

27. Rouwkema, J., et al., Supply of nutrients to cells in engineered tissues. Biotechnol Genet Eng Rev, 2010. 26: p. 163-78.

28. Rouwkema, J., N.C. Rivron, and C.A. van Blitterswijk, Vascularization in tissue engineering. Trends Biotechnol, 2008. 26(8): p. 434-41.

29. Carmeliet, P. and R.K. Jain, Angiogenesis in cancer and other diseases. Nature, 2000. 407(6801): p. 249-57.

30. Zahm, A.M., et al., Numerical modeling of oxygen distributions in cortical and cancellous bone: oxygen availability governs osteonal and trabecular dimensions. Am J Physiol Cell Physiol, 2010. 299(5): p. C922-9.

31. Rouwkema, J. and A. Khademhosseini, Vascularization and Angiogenesis in Tissue Engineering: Beyond Creating Static Networks. Trends Biotechnol, 2016. 34(9): p. 733-745.

32. Santos, M.I. and R.L. Reis, Vascularization in bone tissue engineering: physiology, current strategies, major hurdles and future challenges. Macromol Biosci, 2010. 10(1): p. 12-27.

33. Mohrman, D.E. and L.J. Heller, Chapter 1. Overview of the Cardiovascular System, in Cardiovascular Physiology, 8e. 2014, The McGraw-Hill Companies: New York, NY.

34. Scallan, J., V.H. Huxley, and R.J. Korthuis, Capillary Fluid Exchange: Regulation, Functions, and Pathology, in Regulation of Endothelial Barrier Function. 2010: San Rafael (CA).

35. Yuan, S.Y. and R.R. Rigor, Regulation of Endothelial Barrier Function, in Regulation of Endothelial Barrier Function. 2010: San Rafael (CA).

36. Scallan, J., V.H. Huxley, and R.J. Korthuis, Fluid Movement Across the Endothelial Barrier, in Capillary Fluid Exchange: Regulation, Functions, and Pathology. 2010: San Rafael (CA).

37. Grosso, A., et al., It Takes Two to Tango: Coupling of Angiogenesis and Osteogenesis for Bone Regeneration. Front Bioeng Biotechnol, 2017. 5: p. 68.

38. Percival, C.J. and J.T. Richtsmeier, Angiogenesis and intramembranous osteogenesis. Dev Dyn, 2013. 242(8): p. 909-22.

39. Komori, T., Regulation of Proliferation, Differentiation and Functions of Osteoblasts by Runx2. Int J Mol Sci, 2019. 20(7).

40. Korn, C. and H.G. Augustin, Mechanisms of Vessel Pruning and Regression. Dev Cell, 2015. 34(1): p. 5-17. 
41. Song, H.G., et al., Vascular Tissue Engineering: Progress, Challenges, and Clinical Promise. Cell Stem Cell, 2018. 22(3): p. 340-354.

42. Seifu, D.G., et al., Small-diameter vascular tissue engineering. Nat Rev Cardiol, 2013. 10(7): p. 410-21.

43. Ravi, S., Z. Qu, and E.L. Chaikof, Polymeric materials for tissue engineering of arterial substitutes. Vascular, 2009. 17 Suppl 1: p. S45-54.

44. Chlupac, J., E. Filova, and L. Bacakova, Blood vessel replacement: 50 years of development and tissue engineering paradigms in vascular surgery. Physiol Res, 2009. 58 Suppl 2: p. S119-39.

45. Collins, M.J., et al., Therapeutic strategies to combat neointimal hyperplasia in vascular grafts. Expert Rev Cardiovasc Ther, 2012. 10(5): p. 635-47.

46. Haruguchi, H. and S. Teraoka, Intimal hyperplasia and hemodynamic factors in arterial bypass and arteriovenous grafts: a review. J Artif Organs, 2003. 6(4): p. 227-35.

47. Carrabba, M. and P. Madeddu, Current Strategies for the Manufacture of Small Size Tissue Engineering Vascular Grafts. Front Bioeng Biotechnol, 2018. 6: p. 41.

48. Wang, Z., S.M. Mithieux, and A.S. Weiss, Fabrication Techniques for Vascular and Vascularized Tissue Engineering. Adv Healthc Mater, 2019. 8(19): p. e1900742.

49. Goins, A., A.R. Webb, and J.B. Allen, Multi-layer approaches to scaffold-based small diameter vessel engineering: A review. Mater Sci Eng C Mater Biol Appl, 2019. 97: p. 896-912.

50. McAllister, T.N., et al., Effectiveness of haemodialysis access with an autologous tissue-engineered vascular graft: a multicentre cohort study. Lancet, 2009. 373(9673): p. 1440-6.

51. Wystrychowski, W., et al., Case study: first implantation of a frozen, devitalized tissue-engineered vascular graft for urgent hemodialysis access. J Vasc Access, 2011. 12(1): p. 67-70.

52. Wystrychowski, W., et al., First human use of an allogeneic tissue-engineered vascular graft for hemodialysis access. J Vasc Surg, 2014. 60(5): p. 1353-1357.

53. Shin'oka, T., Y. Imai, and Y. Ikada, Transplantation of a tissue-engineered pulmonary artery. N Engl J Med, 2001. 344(7): p. 532-3.

54. Das, N., et al., Results of a seven-year, single-centre experience of the long-term outcomes of bovine ureter grafts used as novel conduits for haemodialysis fistulas. Cardiovasc Intervent Radiol, 2011. 34(5): p. 958-63.

55. Lindsey, P., et al., Lower Extremity Bypass Using Bovine Carotid Artery Graft (Artegraft): An Analysis of 124 Cases with Long-Term Results. World J Surg, 2018. 42(1): p. 295-301.

56. Lawson, J.H., et al., Bioengineered human acellular vessels for dialysis access in patients with endstage renal disease: two phase 2 single-arm trials. Lancet, 2016. 387(10032): p. 2026-34.

57. Ercolani, E., C. Del Gaudio, and A. Bianco, Vascular tissue engineering of small-diameter blood vessels: reviewing the electrospinning approach. J Tissue Eng Regen Med, 2015. 9(8): p. 861-88.

58. Hasan, A., et al., Electrospun scaffolds for tissue engineering of vascular grafts. Acta Biomater, 2014. 10(1): p. 11-25.

59. Rocco, K.A., et al., In vivo applications of electrospun tissue-engineered vascular grafts: a review. Tissue Eng Part B Rev, 2014. 20(6): p. 628-40. 
60. Chan-Park, M.B., et al., Biomimetic control of vascular smooth muscle cell morphology and phenotype for functional tissue-engineered small-diameter blood vessels. J Biomed Mater Res A, 2009. 88(4): p. 1104-21.

61. Ju, Y.M., et al., Bilayered scaffold for engineering cellularized blood vessels. Biomaterials, 2010. 31(15): p. 4313-21.

62. Wang, Y., et al., Electrospun tubular scaffold with circumferentially aligned nanofibers for regulating smooth muscle cell growth. ACS Appl Mater Interfaces, 2014. 6(4): p. 2958-62.

63. Zhu, M., et al., Circumferentially aligned fibers guided functional neoartery regeneration in vivo. Biomaterials, 2015. 61: p. 85-94.

64. Vaz, C.M., et al., Design of scaffolds for blood vessel tissue engineering using a multi-layering electrospinning technique. Acta Biomater, 2005. 1(5): p. 575-82.

65. Dimitrievska, S. and L.E. Niklason, Historical Perspective and Future Direction of Blood Vessel Developments. Cold Spring Harb Perspect Med, 2018. 8(2).

66. Yousif, L.F., J. Di Russo, and L. Sorokin, Laminin isoforms in endothelial and perivascular basement membranes. Cell Adh Migr, 2013. 7(1): p. 101-10.

67. Attwell, D., et al., What is a pericyte? J Cereb Blood Flow Metab, 2016. 36(2): p. 451-5.

68. Jackson, W.F., Microcirculation, in Muscle. Fundamental Biology and Mechanisms of Disease, E.N.O. Joseph A. Hill, Editor. 2012, Academic Press p. 1197-1206.

69. Blache, U. and M. Ehrbar, Inspired by Nature: Hydrogels as Versatile Tools for Vascular Engineering. Adv Wound Care (New Rochelle), 2018. 7(7): p. 232-246.

70. Klotz, B.J., et al., Gelatin-Methacryloyl Hydrogels: Towards Biofabrication-Based Tissue Repair. Trends Biotechnol, 2016. 34(5): p. 394-407.

71. Barabaschi, G.D., et al., Engineering Pre-vascularized Scaffolds for Bone Regeneration. Adv Exp Med Biol, 2015. 881: p. 79-94.

72. Lebaschi, A., et al., Tissue-specific endothelial cells: a promising approach for augmentation of soft tissue repair in orthopedics. Ann N Y Acad Sci, 2017. 1410(1): p. 44-56.

73. Kocherova, I., et al., Human Umbilical Vein Endothelial Cells (HUVECS) Co-Culture with Osteogenic Cells: From Molecular Communication to Engineering Prevascularised Bone Grafts. J Clin Med, 2019. 8(10).

74. Haug, V., et al., Comparison between endothelial progenitor cells and human umbilical vein endothelial cells on neovascularization in an adipogenesis mouse model. Microvasc Res, 2015. 97: p. 159-66.

75. Liang, T., et al., Coculture of endothelial progenitor cells and mesenchymal stem cells enhanced their proliferation and angiogenesis through PDGF and Notch signaling. FEBS Open Bio, 2017. 7(11): p. 1722-1736.

76. Peters, E.B., Endothelial Progenitor Cells for the Vascularization of Engineered Tissues. Tissue Eng Part B Rev, 2018. 24(1): p. 1-24.

77. Kusuma, S., et al., Self-organized vascular networks from human pluripotent stem cells in a synthetic matrix. Proc Natl Acad Sci U S A, 2013. 110(31): p. 12601-6. 
78. Samuel, R., et al., Generation of functionally competent and durable engineered blood vessels from human induced pluripotent stem cells. Proc Natl Acad Sci U S A, 2013. 110(31): p. 12774-9.

79. Darland, D.C., et al., Pericyte production of cell-associated VEGF is differentiation-dependent and is associated with endothelial survival. Dev Biol, 2003. 264(1): p. 275-88.

80. Korff, T., et al., Blood vessel maturation in a 3-dimensional spheroidal coculture model: direct contact with smooth muscle cells regulates endothelial cell quiescence and abrogates VEGF responsiveness. FASEB J, 2001. 15(2): p. 447-57.

81. Pennings, I., et al., Effect of donor variation on osteogenesis and vasculogenesis in hydrogel cocultures. J Tissue Eng Regen Med, 2019.

82. Frohlich, M., et al., Tissue engineered bone grafts: biological requirements, tissue culture and clinical relevance. Curr Stem Cell Res Ther, 2008. 3(4): p. 254-64.

83. Guerrero, J., et al., Cell interactions between human progenitor-derived endothelial cells and human mesenchymal stem cells in a three-dimensional macroporous polysaccharide-based scaffold promote osteogenesis. Acta Biomater, 2013. 9(9): p. 8200-13.

84. Unger, R.E., et al., Tissue-like self-assembly in cocultures of endothelial cells and osteoblasts and the formation of microcapillary-like structures on three-dimensional porous biomaterials. Biomaterials, 2007. 28(27): p. 3965-76.

85. Liu, Y., et al., Vasculogenic and osteogenesis-enhancing potential of human umbilical cord blood endothelial colony-forming cells. Stem Cells, 2012. 30(9): p. 1911-24.

86. Li, Q. and Z. Wang, Influence of mesenchymal stem cells with endothelial progenitor cells in coculture on osteogenesis and angiogenesis: an in vitro study. Arch Med Res, 2013. 44(7): p. 504-13.

87. Mercado-Pagan, A.E., et al., Vascularization in bone tissue engineering constructs. Ann Biomed Eng, 2015. 43(3): p. 718-29.

88. Akita, S., et al., Capillary vessel network integration by inserting a vascular pedicle enhances bone formation in tissue-engineered bone using interconnected porous hydroxyapatite ceramics. Tissue Eng, 2004. 10(5-6): p. 789-95.

89. Sever, C., et al., Prefabrication of vascularized bone graft using an interconnected porous calcium hydroxyapatite ceramic in presence of vascular endothelial growth factor and bone marrow mesenchymal stem cells: Experimental study in rats. Indian J Plast Surg, 2012. 45(3): p. 444-52.

90. Beier, J.P., et al., Axial vascularization of a large volume calcium phosphate ceramic bone substitute in the sheep AV loop model. J Tissue Eng Regen Med, 2010. 4(3): p. 216-23.

91. Weigand, A., et al., Acceleration of vascularized bone tissue-engineered constructs in a large animal model combining intrinsic and extrinsic vascularization. Tissue Eng Part A, 2015. 21(9-10): p. 1680-94.

92. Arkudas, A., et al., Fibrin gel-immobilized VEGF and bFGF efficiently stimulate angiogenesis in the AV loop model. Mol Med, 2007. 13(9-10): p. 480-7.

93. Buehrer, G., et al., Combination of BMP2 and MSCs significantly increases bone formation in the rat arterio-venous loop model. Tissue Eng Part A, 2015. 21(1-2): p. 96-105.

94. Arkudas, A., et al., Axial prevascularization of porous matrices using an arteriovenous loop promotes survival and differentiation of transplanted autologous osteoblasts. Tissue Eng, 2007. 13(7): p. 1549-60. 
95. Arkudas, A., et al., Composition of fibrin glues significantly influences axial vascularization and degradation in isolation chamber model. Blood Coagul Fibrinolysis, 2012. 23(5): p. 419-27.

96. Beier, J.P., et al., De novo generation of axially vascularized tissue in a large animal model. Microsurgery, 2009. 29(1): p. 42-51.

97. Eweida, A.M., et al., Axially vascularised mandibular constructs: Is it time for a clinical trial? J Craniomaxillofac Surg, 2015. 43(7): p. 1028-32.

98. Eweida, A.M., et al., Mandibular reconstruction using an axially vascularized tissue-engineered construct. Ann Surg Innov Res, 2011. 5: p. 2.

99. Hoang, N.T., et al., Prefabrication of large fasciocutaneous flaps using an isolated arterialised vein as implanted vascular pedicle. Br J Plast Surg, 2005. 58(5): p. 632-9.

100. Kneser, U., et al., Engineering of vascularized transplantable bone tissues: induction of axial vascularization in an osteoconductive matrix using an arteriovenous loop. Tissue Eng, 2006. 12(7): p. 1721-31.

101. Eweida, A., et al., Axially vascularized tissue-engineered bone constructs retain their in vivo angiogenic and osteogenic capacity after high-dose irradiation. J Tissue Eng Regen Med, 2018. 12(2): p. e657-e668.

102. Horch, R.E., et al., Successful human long-term application of in situ bone tissue engineering. J Cell Mol Med, 2014. 18(7): p. 1478-85.

103. Klotz, B.J., et al., Engineering of a complex bone tissue model with endothelialised channels and capillary-like networks. Eur Cell Mater, 2018. 35: p. 335-348.

104. Kolesky, D.B., et al., Three-dimensional bioprinting of thick vascularized tissues. Proc Natl Acad Sci U S A, 2016. 113(12): p. 3179-84.

105. Miller, J.S., et al., Rapid casting of patterned vascular networks for perfusable engineered threedimensional tissues. Nat Mater, 2012. 11(9): p. 768-74.

106. Nguyen, D.H., et al., Biomimetic model to reconstitute angiogenic sprouting morphogenesis in vitro. Proc Natl Acad Sci U S A, 2013. 110(17): p. 6712-7.

107. Wang, X., Q. Sun, and J. Pei, Microfluidic-Based 3D Engineered Microvascular Networks and Their Applications in Vascularized Microtumor Models. Micromachines (Basel), 2018. 9(10).

108. Wang, X., et al., Engineering anastomosis between living capillary networks and endothelial celllined microfluidic channels. Lab Chip, 2016. 16(2): p. 282-90.

109. Lee, V.K., et al., Generation of Multi-Scale Vascular Network System within 3D Hydrogel using 3D Bio-Printing Technology. Cell Mol Bioeng, 2014. 7(3): p. 460-472.

110. Whited, B.M. and M.N. Rylander, The influence of electrospun scaffold topography on endothelial cell morphology, alignment, and adhesion in response to fluid flow. Biotechnol Bioeng, 2014. 111(1): p. 184-95.

111. Zilla, P., D. Bezuidenhout, and P. Human, Prosthetic vascular grafts: wrong models, wrong questions and no healing. Biomaterials, 2007. 28(34): p. 5009-27.

112. Thottappillil, N. and P.D. Nair, Scaffolds in vascular regeneration: current status. Vasc Health Risk Manag, 2015. 11: p. 79-91.

113. Ravi, S. and E.L. Chaikof, Biomaterials for vascular tissue engineering. Regen Med, 2010. 5(1): p. 107-20. 
114. L'Heureux, N., et al., A completely biological tissue-engineered human blood vessel. FASEB J, 1998. 12(1): p. 47-56.

115. L'Heureux, N., et al., Human tissue-engineered blood vessels for adult arterial revascularization. Nat Med, 2006. 12(3): p. 361-5.

116. Isenberg, B.C., C. Williams, and R.T.Tranquillo, Small-diameter artificial arteries engineered in vitro. Circ Res, 2006. 98(1): p. 25-35.

117. Syedain, Z., et al., Tissue engineering of acellular vascular grafts capable of somatic growth in young lambs. Nat Commun, 2016. 7: p. 12951.

118. Morin, K.T., J.L. Dries-Devlin, and R.T. Tranquillo, Engineered microvessels with strong alignment and high lumen density via cell-induced fibrin gel compaction and interstitial flow. Tissue Eng Part A, 2014. 20(3-4): p. 553-65.

119. Koch, S., et al., Fibrin-polylactide-based tissue-engineered vascular graft in the arterial circulation. Biomaterials, 2010. 31(17): p. 4731-9.

120. Tschoeke, B., et al., Tissue-engineered small-caliber vascular graft based on a novel biodegradable composite fibrin-polylactide scaffold. Tissue Eng Part A, 2009. 15(8): p. 1909-18.

121. Narayan, D. and S.S. Venkatraman, Effect of pore size and interpore distance on endothelial cell growth on polymers. J Biomed Mater Res A, 2008. 87(3): p. 710-8.

122.Zhang, Y., et al., Electrospinning of gelatin fibers and gelatin/PCL composite fibrous scaffolds. J Biomed Mater Res B Appl Biomater, 2005. 72(1): p. 156-65.

123. Jungst, T., et al., Melt electrospinning onto cylinders: effects of rotational velocity and collector diameter on morphology of tubular structures. Polymer International, 2015. 64(9): p. 1086-1095.

124. Brown, T.D., P.D. Dalton, and D.W. Hutmacher, Melt electrospinning today: An opportune time for an emerging polymer process. Progress in Polymer Science, 2016. 56: p. 116-166.

125. Brown, T.D., P.D. Dalton, and D.W. Hutmacher, Direct writing by way of melt electrospinning. Advanced Materials, 2011. 23(47): p. 5651-7.

126. Brown, T.D., et al., Design and fabrication of tubular scaffolds via direct writing in a melt electrospinning mode. Biointerphases, 2012. 7(1-4): p. 1-16.

127. Dalton, P.D., et al., Patterned melt electrospun substrates for tissue engineering. Biomedical Materials, 2008. 3(3): p. 034109.

128. Hochleitner, G., et al., Additive manufacturing of scaffolds with sub-micron filaments via melt electrospinning writing. Biofabrication, 2015. 7(3): p. 035002.

129. Hrynevich, A., et al., Dimension-Based Design of Melt Electrowritten Scaffolds. Small, 2018. 14(22): p. e1800232.

130. McColl, E., et al., Design and fabrication of melt electrowritten tubes using intuitive software. Materials \& Design, 2018. 155: p. 46-58.

131. Driessen, N.J., et al., A computational model for collagen fibre remodelling in the arterial wall. J Theor Biol, 2004. 226(1): p. 53-64.

132. Hedin, U., et al., Control of smooth muscle cell proliferation--the role of the basement membrane. Thromb Haemost, 1999. 82 Suppl 1: p. 23-6. 
133. Huang, A.H. and L.E. Niklason, Engineering of arteries in vitro. Cell Mol Life Sci, 2014. 71(11): p. 2103-18.

134. Caves, J.M., et al., The use of microfiber composites of elastin-like protein matrix reinforced with synthetic collagen in the design of vascular grafts. Biomaterials, 2010. 31(27): p. 7175-82.

135. Agrawal, A., etal.,SmoothMuscle CellAlignmentandPhenotype Controlby MeltSpun Polycaprolactone Fibers for Seeding of Tissue Engineered Blood Vessels. Int J Biomater, 2015. 2015: p. 434876.

136.Ju, Y.M., et al., Electrospun vascular scaffold for cellularized small diameter blood vessels: $A$ preclinical large animal study. Acta Biomater, 2017. 59: p. 58-67.

137. Yuan, B., et al., A strategy for depositing different types of cells in three dimensions to mimic tubular structures in tissues. Adv Mater, 2012. 24(7): p. 890-6.

138. Deepthi, S., et al., Engineering poly(hydroxy butyrate-co-hydroxy valerate) based vascular scaffolds to mimic native artery. Int J Biol Macromol, 2018. 109: p. 85-98.

139. Nam, J., et al., Improved cellular infiltration in electrospun fiber via engineered porosity. Tissue Eng, 2007. 13(9): p. 2249-57.

140. Goumans, M.J., et al., Activin receptor-like kinase (ALK)1 is an antagonistic mediator of lateral TGFbeta/ALK5 signaling. Mol Cell, 2003. 12(4): p. 817-28.

141.Zhang, Y., et al., AMP-activated protein kinase is involved in endothelial NO synthase activation in response to shear stress. Arterioscler Thromb Vasc Biol, 2006. 26(6): p. 1281-7.

142. Lim, S.H., et al., Tissue-engineered blood vessels with endothelial nitric oxide synthase activity. J Biomed Mater Res B Appl Biomater, 2008. 85(2): p. 537-46.

143. Liu, D., et al., Bioinspired 3D Multilayered Shape Memory Scaffold with a Hierarchically Changeable Micropatterned Surface for Efficient Vascularization. ACS Appl Mater Interfaces, 2017. 9(23): p. 19725-19735.

144. Liu, X., et al., Study of composite vascular scaffold combining with differentiated VSMC- and VEClike cells in vitro and in vivo. J Biomater Appl, 2017. 32(2): p. 219-229.

145. Nakayama, K.H., et al., Bilayered vascular graft derived from human induced pluripotent stem cells with biomimetic structure and function. Regen Med, 2015. 10(6): p. 745-55.

146. Ye, L., et al., The fabrication of double layer tubular vascular tissue engineering scaffold via coaxial electrospinning and its 3D cell coculture. J Biomed Mater Res A, 2015. 103(12): p. 3863-71.

147. Liu, Y., et al., Engineering blood vessels through micropatterned co-culture of vascular endothelial and smooth muscle cells on bilayered electrospun fibrous mats with pDNA inoculation. Acta Biomater, 2015. 11: p. 114-25.

148. Owens, G.K., Regulation of differentiation of vascular smooth muscle cells. Physiol Rev, 1995. 75(3): p. 487--517.

149. Abedin, M., Y. Tintut, and L.L. Demer, Mesenchymal stem cells and the artery wall. Circ Res, 2004. 95(7): p. 671-6.

150. Gojo, S., et al., In vivo cardiovasculogenesis by direct injection of isolated adult mesenchymal stem cells. Exp Cell Res, 2003. 288(1): p. 51-9.

151. Hirschi, K.K. and M.W. Majesky, Smooth muscle stem cells. Anat Rec A Discov Mol Cell Evol Biol, 2004. 276(1): p. 22-33. 
152. Gong, Z., et al., Influence of culture medium on smooth muscle cell differentiation from human bone marrow-derived mesenchymal stem cells. Tissue Eng Part A, 2009. 15(2): p. 319-30.

153. Gong, Z. and L.E. Niklason, Small-diameter human vessel wall engineered from bone marrowderived mesenchymal stem cells (hMSCS). FASEB J, 2008. 22(6): p. 1635-48.

154. Hirschi, K.K., D.A. Ingram, and M.C. Yoder, Assessing identity, phenotype, and fate of endothelial progenitor cells. Arterioscler Thromb Vasc Biol, 2008. 28(9): p. 1584-95.

155. Ingram, D.A., et al., Identification of a novel hierarchy of endothelial progenitor cells using human peripheral and umbilical cord blood. Blood, 2004. 104(9): p. 2752-60.

156. Albelda, S.M., et al., Molecular and cellular properties of PECAM-1 (endoCAM/CD31): a novel vascular cell-cell adhesion molecule. J Cell Biol, 1991. 114(5): p. 1059-68.

157. Gavard, J., Endothelial permeability and VE-cadherin: a wacky comradeship. Cell Adh Migr, 2014. 8(2): p. 158-64.

158. Lertkiatmongkol, P., et al., Endothelial functions of platelet/endothelial cell adhesion molecule-1 (CD31). Curr Opin Hematol, 2016. 23(3): p. 253-9.

159. Arends, F., et al., The biophysical properties of Basal lamina gels depend on the biochemical composition of the gel. PLoS One, 2015. 10(2): p. e0118090.

160. Khazaei, M., F. Moien-Afshari, and I. Laher, Vascular endothelial function in health and diseases. Pathophysiology, 2008. 15(1): p. 49-67.

161. Seeger, T., et al., Mesenchymal Stromal Cells for Sphincter Regeneration: Role of Laminin Isoforms upon Myogenic Differentiation. PLoS One, 2015. 10(9): p. e0137419.

162. Holzapfel, G.A., T.C. Gasser, and M. Stadler, A structural model for the viscoelastic behavior of arterial walls: continuum formulation and finite element analysis. European Journal of Mechanics-A/ Solids, 2002. 21(3): p. 441-463.

163. Spronck, B., et al., A method for three-dimensional quantification of vascular smooth muscle orientation: application in viable murine carotid arteries. Biomech Model Mechanobiol, 2016. 15(2): p. 419-32.

164.Gu, W., et al., Smooth muscle cells differentiated from mesenchymal stem cells are regulated by microRNAs and suitable for vascular tissue grafts. J Biol Chem, 2018. 293(21): p. 8089-8102.

165. Beamish, J.A., et al., Molecular regulation of contractile smooth muscle cell phenotype: implications for vascular tissue engineering. Tissue Eng Part B Rev, 2010. 16(5): p. 467-91.

166. Rovner, A.S., R.A. Murphy, and G.K. Owens, Expression of smooth muscle and nonmuscle myosin heavy chains in cultured vascular smooth muscle cells. J Biol Chem, 1986. 261(31): p. 14740-5.

167.Zhong, J., et al., Effect of nanofiber orientation of electrospun nanofibrous scaffolds on cell growth and elastin expression of muscle cells. Colloids Surf B Biointerfaces, 2015. 136: p. 772-8.

168. Yuan, H., et al., Highly aligned core-shell structured nanofibers for promoting phenotypic expression of vSMCs for vascular regeneration. Nanoscale, 2016. 8(36): p. 16307-16322.

169. Cao, Y., et al., Regulating orientation and phenotype of primary vascular smooth muscle cells by biodegradable films patterned with arrays of microchannels and discontinuous microwalls. Biomaterials, 2010. 31(24): p. 6228-38. 
170. Shen, J.Y., et al., Three-dimensional microchannels in biodegradable polymeric films for control orientation and phenotype of vascular smooth muscle cells. Tissue Eng, 2006. 12(8): p. 2229-40.

171. Alimperti, S., et al., Cadherin-11 regulates both mesenchymal stem cell differentiation into smooth muscle cells and the development of contractile function in vivo. J Cell Sci, 2014. 127(Pt 12): p. 2627-38.

172. Espagnolle, N., et al., CD146 expression on mesenchymal stem cells is associated with their vascular smooth muscle commitment. Journal of Cellular and Molecular Medicine, 2014. 18(1): p. 104-114.

173. Gokcinar-Yagci, B. and B. Celebi-Saltik, Comparison of different culture conditions for smooth muscle cell differentiation of human umbilical cord vein CD146+ perivascular cells. Cell Tissue Bank, 2017. 18(4): p. 501-511.

174. Liu, Y., et al., Differentiated markers in undifferentiated cells: expression of smooth muscle contractile proteins in multipotent bone marrow mesenchymal stem cells. Dev Growth Differ, 2013. 55(5): p. 591-605.

175. Pfaffl, M.W., A new mathematical model for relative quantification in real-time RT-PCR. Nucleic Acids Res, 2001. 29(9): p. e45.

176. Pfaffl, M.W., G.W. Horgan, and L. Dempfle, Relative expression software tool (REST) for group-wise comparison and statistical analysis of relative expression results in real-time PCR. Nucleic Acids Res, 2002. 30(9): p. e36.

177. Kim, M.R., et al., Thromboxane a(2) induces differentiation of human mesenchymal stem cells to smooth muscle-like cells. Stem Cells, 2009. 27(1): p. 191-9.

178. Salacinski, H.J., et al., The mechanical behavior of vascular grafts: a review. J Biomater Appl, 2001. 15(3): p. 241-78.

179.Gui, L. and L.E. Niklason, Vascular Tissue Engineering: Building Perfusable Vasculature for Implantation. Curr Opin Chem Eng, 2014. 3: p. 68-74.

180. Hasan, A., et al., Electrospun scaffolds for tissue engineering of vascular grafts. Acta Biomater, 2014. 10(1): p. 11--25.

181. Woods, I. and T.C. Flanagan, Electrospinning of biomimetic scaffolds for tissue-engineered vascular grafts: threading the path. Expert Rev Cardiovasc Ther, 2014. 12(7): p. 815-32.

182. Jungst, T., et al., Heterotypic Scaffold Design Orchestrates Primary Cell Organization and Phenotypes in Cocultured Small Diameter Vascular Grafts. Advanced Functional Materials, 2019. 29(43).

183. Davies, P.F., J. Zilberberg, and B.P. Helmke, Spatial microstimuli in endothelial mechanosignaling. Circ Res, 2003. 92(4): p. 359-70.

184. Lu, D. and G.S. Kassab, Role of shear stress and stretch in vascular mechanobiology. J R Soc Interface, 2011. 8(63): p. 1379-85.

185. Egorova, A.D., et al., Endothelial colony-forming cells show a mature transcriptional response to shear stress. In Vitro Cell Dev Biol Anim, 2012. 48(1): p. 21-9.

186. Obi, S., K. Yamamoto, and J. Ando, Effects of shear stress on endothelial progenitor cells. J Biomed Nanotechnol, 2014. 10(10): p. 2586--2597.

187. Obi, S., et al., Fluid shear stress induces arterial differentiation of endothelial progenitor cells. J Appl Physiol (1985), 2009. 106(1): p. 203-11. 
188. Yamamoto, K., et al., Proliferation, differentiation, and tube formation by endothelial progenitor cells in response to shear stress. J Appl Physiol, 2003. 95(5): p. 2081--2088.

189. Inoguchi, $\mathrm{H}_{\text {., }}$ et al., The effect of gradually graded shear stress on the morphological integrity of a huvec-seeded compliant small-diameter vascular graft. Biomaterials, 2007. 28(3): p. 486--495.

190. Koo, M.A., et al., Stimulated migration and penetration of vascular endothelial cells into poly (L-lactic acid) scaffolds under flow conditions. Biomater Res, 2014. 18: p. 7.

191. Kwak, B.R., et al., Shear stress and cyclic circumferential stretch, but not pressure, alter connexin43 expression in endothelial cells. Cell Commun Adhes, 2005. 12(5-6): p. 261--270.

192. Levesque, M.J. and R.M. Nerem, The Elongation and Orientation of Cultured Endothelial Cells in Response to Shear Stress. J Biomech Eng, 1985. 107(4): p. 341--347.

193. Nackman, G.B., et al., Flow modulates endothelial regulation of smooth muscle cell proliferation: a new model. Surgery, 1998. 124(2): p. 353-60; discussion 360-1.

194. Owatverot, T.B., et al., Effect of combined cyclic stretch and fluid shear stress on endothelial cell morphological responses. J Biomech Eng, 2005. 127(3): p. 374-82.

195.Zeng, Y. and J.M. Tarbell, The adaptive remodeling of endothelial glycocalyx in response to fluid shear stress. PLoS One, 2014. 9(1): p. 1--15.

196.Zhang, J. and M.H. Friedman, Adaptive response of vascular endothelial cells to an acute increase in shear stress frequency. Am J Physiol Heart Circ Physiol, 2013. 305(6): p. H894-902.

197. Dekker, R.J., et al., Prolonged fluid shear stress induces a distinct set of endothelial cell genes, most specifically lung Kruppel-like factor (KLF2). Blood, 2002. 100(5): p. 1689-98.

198. Kaushal, S., et al., Functional small-diameter neovessels created using endothelial progenitor cells expanded ex vivo. Nat Med, 2001. 7(9): p. 1035-40.

199. Niklason, L.E., et al., Functional arteries grown in vitro. Science, 1999. 284(5413): p. 489-93.

200. Lee, S.J., et al., Development of a composite vascular scaffolding system that withstands physiological vascular conditions. Biomaterials, 2008. 29(19): p. 2891-8.

201. Ott, M.J. and B.J. Ballermann, Shear stress-conditioned, endothelial cell-seeded vascular grafts: improved cell adherence in response to in vitro shear stress. Surgery, 1995. 117(3): p. 334-9.

202. Elliott, M.B. and S. Gerecht, Three-dimensional culture of small-diameter vascular grafts. Journal of Materials Chemistry B, 2016. 4(20): p. 3443-3453.

203.de Jonge, N., et al., Strain-induced collagen organization at the micro-level in fibrin-based engineered tissue constructs. Ann Biomed Eng, 2013. 41(4): p. 763-74.

204. Fioretta, E.S., et al., Polymer-based scaffold designs for in situ vascular tissue engineering: controlling recruitment and differentiation behavior of endothelial colony forming cells. Macromol Biosci, 2012. 12(5): p. 577-90.

205. White, F.M., Fluid Mechanics. 2011. seventh edition p. $272-273$.

206. Pennings, I., et al., Effect of donor variation on osteogenesis and vasculogenesis in hydrogel cocultures. J Tissue Eng Regen Med, 2019. 13(3): p. 433-445.

207. van Haaften, E.E., et al., Decoupling the Effect of Shear Stress and Stretch on Tissue Growth and Remodeling in a Vascular Graft. Tissue Eng Part C Methods, 2018. 24(7): p. 418-429. 
208. Malek, A.M., S.L. Alper, and S. Izumo, Hemodynamic shear stress and its role in atherosclerosis. JAMA, 1999. 282(21): p. 2035-42.

209. van den Broek, C.N., et al., Medium with blood-analog mechanical properties for cardiovascular tissue culturing. Biorheology, 2008. 45(6): p. 651-61.

210. Privratsky, J.R. and P.J. Newman, PECAM-1: regulator of endothelial junctional integrity. Cell Tissue Res, 2014. 355(3): p. 607-19.

211. Baeyens, N. and M.A. Schwartz, Biomechanics of vascular mechanosensation and remodeling. Mol Biol Cell, 2016. 27(1): p. 7--11.

212. Tzima, E., et al., A mechanosensory complex that mediates the endothelial cell response to fluid shear stress. Nature, 2005. 437(7057): p. 426--431.

213. Barry, A.K., N. Wang, and D.E. Leckband, Local VE-cadherin mechanotransduction triggers longranged remodeling of endothelial monolayers. J Cell Sci, 2015. 128(7): p. 1341-51.

214. Baeyens, N., et al., Vascular remodeling is governed by a VEGFR3-dependent fluid shear stress set point. Elife, 2015. 4.

215. Ohta, S., S. Inasawa, and Y. Yamaguchi, Alignment of vascular endothelial cells as a collective response to shear flow. J Phys D Appl Phys, 2015. 48(24).

216. Di Russo, J., et al., Endothelial basement membrane laminin 511 is essential for shear stress response. EMBO J, 2017. 36(2): p. 183-201.

217. Girard, P.R. and R.M. Nerem, Shear stress modulates endothelial cell morphology and F-actin organization through the regulation of focal adhesion-associated proteins. J Cell Physiol, 1995. 163(1): p. 179-93.

218. Verma, D., et al., Flow-induced focal adhesion remodeling mediated by local cytoskeletal stresses and reorganization. Cell Adh Migr, 2015. 9(6): p. 432--440.

219. Siedlecki, C.A., et al., Shear-dependent changes in the three-dimensional structure of human von Willebrand factor. Blood, 1996. 88(8): p. 2939-50.

220. Tsai, H.M., Shear stress and von Willebrand factor in health and disease. Semin Thromb Hemost, 2003. 29(5): p. 479-88.

221. Auton, M., C. Zhu, and M.A. Cruz, The mechanism of VWF-mediated platelet GPIbalpha binding. Biophys J, 2010. 99(4): p. 1192-201.

222. SenBanerjee, S., et al., KLF2 Is a novel transcriptional regulator of endothelial proinflammatory activation. J Exp Med, 2004. 199(10): p. 1305-15.

223. Brown, D.J., et al., Endothelial cell activation of the smooth muscle cell phosphoinositide 3-kinase/ Akt pathway promotes differentiation. J Vasc Surg, 2005. 41(3): p. 509-16.

224. Hirschi, K. and P.A. D'Amore, Pericytes in the microvasculature. Cardiovasc Res, 1996. 32(4): p. 687--698.

225. Lilly, B., We have contact: endothelial cell-smooth muscle cell interactions. Physiology (Bethesda), 2014. 29(4): p. 234-41.

226. Wang, G., et al., Origin and differentiation of vascular smooth muscle cells. J Physiol, 2015. 593(14): p. 3013--3030.

227. Gong, Z., et al., Influence of Culture Medium on Smooth Muscle Cell. Tissue Eng Part A, 2009. 15(2): p. 319--330. 
228. Bacakova, L., et al., The Role of Vascular Smooth Muscle Cells in the Physiology and Pathophysiology of Blood Vessels. 2018: p. 229--257.

229. Owens, G.K., M.S. Kumar, and B.R. Wamhoff, Molecular Regulation of Vascular Smooth Muscle Cell Differentiation in Development and Disease. Physiol Rev, 2004. 84(3): p. 767--801.

230. Hanson, A.N. and J.P. Bentley, Quantitation of type I to type III collagen ratios in small samples of human tendon, blood vessels, and atherosclerotic plaque. Anal Biochem, 1983. 130(1): p. 32-40.

231. van Haaften, E.E., C.V.C. Bouten, and N.A. Kurniawan, Vascular Mechanobiology: Towards Control of In Situ Regeneration. Cells, 2017. 6(3).

232. Wolf, F., et al., Bioengineered vascular constructs as living models for linvitro cardiovascular research. Drug Discov Today, 2016. 21(9): p. 1446--1455.

233. Dimitriou, R., et al., Complications following autologous bone graft harvesting from the iliac crest and using the RIA: a systematic review. Injury, 2011. 42 Suppl 2: p. S3-15.

234. Ross, N., L. Tacconi, and J.B. Miles, Heterotopic bone formation causing recurrent donor site pain following iliac crest bone harvesting. Br J Neurosurg, 2000. 14(5): p. 476-9.

235. Liu, Y., J.K. Chan, and S.H. Teoh, Review of vascularised bone tissue-engineering strategies with a focus on co-culture systems. JTissue Eng Regen Med, 2015. 9(2): p. 85-105.

236. Banno, K. and M.C. Yoder, Tissue regeneration using endothelial colony-forming cells: promising cells for vascular repair. Pediatr Res, 2018. 83(1-2): p. 283-290.

237. Gawlitta, D., et al., Hypoxia impedes vasculogenesis of in vitro engineered bone. Tissue Eng Part A, 2012. 18(1-2): p. 208-18.

238. Katsara, O., et al., Effects of donor age, gender, and in vitro cellular aging on the phenotypic, functional, and molecular characteristics of mouse bone marrow-derived mesenchymal stem cells. Stem Cells Dev, 2011. 20(9): p. 1549-61.

239. Schellenberg, A., et al., Replicative senescence of mesenchymal stem cells causes DNA-methylation changes which correlate with repressive histone marks. Aging (Albany NY), 2011. 3(9): p. 873-88.

240. Bertram, H., H. Mayer, and H. Schliephake, Effect of donor characteristics, technique of harvesting and in vitro processing on culturing of human marrow stroma cells for tissue engineered growth of bone. Clin Oral Implants Res, 2005. 16(5): p. 524-31.

241. Siddappa, R., et al., Donor variation and loss of multipotency during in vitro expansion of human mesenchymal stem cells for bone tissue engineering. J Orthop Res, 2007. 25(8): p. 1029-41.

242. Phinney, D.G., et al., Donor variation in the growth properties and osteogenic potential of human marrow stromal cells. J Cell Biochem, 1999. 75(3): p. 424-36.

243. Stenderup, K., et al., Aging is associated with decreased maximal life span and accelerated senescence of bone marrow stromal cells. Bone, 2003. 33(6): p. 919-26.

244. Portalska, K.J., et al., The effect of donor variation and senescence on endothelial differentiation of human mesenchymal stromal cells. Tissue Eng Part A, 2013. 19(21-22): p. 2318-29.

245. Mund, J.A., et al., Flow cytometric identification and functional characterization of immature and mature circulating endothelial cells. Arterioscler Thromb Vasc Biol, 2012. 32(4): p. 1045-53.

246. Richardson, M.R. and M.C. Yoder, Endothelial progenitor cells: quo vadis? J Mol Cell Cardiol, 2011. 50(2): p. 266-72. 
247. Barruet, E., et al., The ACVR1 R206H mutation found in fibrodysplasia ossificans progressiva increases human induced pluripotent stem cell-derived endothelial cell formation and collagen production through BMP-mediated SMAD1/5/8 signaling. Stem Cell Res Ther, 2016. 7(1): p. 115.

248. White, M.P., et al., Limited gene expression variation in human embryonic stem cell and induced pluripotent stem cell-derived endothelial cells. Stem Cells, 2013. 31(1): p. 92-103.

249. Matsumoto, Y., et al., Induced pluripotent stem cells from patients with human fibrodysplasia ossificans progressiva show increased mineralization and cartilage formation. Orphanet J Rare Dis, 2013. 8: p. 190.

250. Theodoris, C.V., et al., Human disease modeling reveals integrated transcriptional and epigenetic mechanisms of NOTCH1 haploinsufficiency. Cell, 2015. 160(6): p. 1072-86.

251. Niemisto, A., et al., Robust quantification of in vitro angiogenesis through image analysis. IEEE Trans Med Imaging, 2005. 24(4): p. 549-53.

252. Dominici, M., et al., Minimal criteria for defining multipotent mesenchymal stromal cells. The International Society for Cellular Therapy position statement. Cytotherapy, 2006. 8(4): p. 315-7.

253. Qu, Z., et al., The initial attachment and subsequent behavior regulation of osteoblasts by dental implant surface modification. J Biomed Mater Res A, 2007. 82(3): p. 658-68.

254. Nassiri, S.M. and R. Rahbarghazi, Interactions of mesenchymal stem cells with endothelial cells. Stem Cells Dev, 2014. 23(4): p. 319-32.

255. Bidarra, S.J., et al., Phenotypic and proliferative modulation of human mesenchymal stem cells via crosstalk with endothelial cells. Stem Cell Res, 2011. 7(3): p. 186-97.

256. Loibl, M., et al., Direct cell-cell contact between mesenchymal stem cells and endothelial progenitor cells induces a pericyte-like phenotype in vitro. Biomed Res Int, 2014. 2014: p. 395781.

257. Kyllonen, L., et al., Effects of different serum conditions on osteogenic differentiation of human adipose stem cells in vitro. Stem Cell Res Ther, 2013. 4(1): p. 17.

258. Ogston, N., et al., Dexamethasone and retinoic acid differentially regulate growth and differentiation in an immortalised human clonal bone marrow stromal cell line with osteoblastic characteristics. Steroids, 2002. 67(11): p. 895-906.

259. Cellular Dynamics International Inc. Cellular Dynamics Manufactures CGMP HLA "Superdonor" Stem Cell Lines to Enable Cell Therapy With Genetic Matching. [Website ] 2015 February 9, 2015 [cited 2017 July 31].

260. Belair, D.G., et al., Human vascular tissue models formed from human induced pluripotent stem cell derived endothelial cells. Stem Cell Rev, 2015. 11(3): p. 511-25.

261. Margariti, A., et al., Direct reprogramming of fibroblasts into endothelial cells capable of angiogenesis and reendothelialization in tissue-engineered vessels. Proc Natl Acad Sci U S A, 2012. 109(34): p. 13793-8.

262. Zanotelli, M.R., et al., Stable engineered vascular networks from human induced pluripotent stem cell-derived endothelial cells cultured in synthetic hydrogels. Acta Biomater, 2016. 35: p. 32-41.

263. Orlova, V.V., et al., Functionality of endothelial cells and pericytes from human pluripotent stem cells demonstrated in cultured vascular plexus and zebrafish xenografts. Arterioscler Thromb Vasc Biol, 2014. 34(1): p. 177-86. 
264. Chan, X.Y., et al., Three-Dimensional Vascular Network Assembly From Diabetic Patient-Derived Induced Pluripotent Stem Cells. Arterioscler Thromb Vasc Biol, 2015. 35(12): p. 2677-85.

265. Eliaz, N. and N. Metoki, Calcium Phosphate Bioceramics: A Review of Their History, Structure, Properties, Coating Technologies and Biomedical Applications. Materials (Basel), 2017. 10(4).

266. Habraken, W., et al., Calcium phosphates in biomedical applications: materials for the future? Materials Today, 2016. 19(2): p. 69-87.

267. Barrere, F., C.A. van Blitterswijk, and K. de Groot, Bone regeneration: molecular and cellular interactions with calcium phosphate ceramics. Int J Nanomedicine, 2006. 1(3): p. 317-32.

268. Fernandez de Grado, G., et al., Bone substitutes: a review of their characteristics, clinical use, and perspectives for large bone defects management. J Tissue Eng, 2018. 9: p. 2041731418776819.

269. Barradas, A.M., et al., Osteoinductive biomaterials: current knowledge of properties, experimental models and biological mechanisms. Eur Cell Mater, 2011. 21: p. 407-29; discussion 429.

270. Wang, L., et al., Ectopic osteoid and bone formation by three calcium-phosphate ceramics in rats, rabbits and dogs. PLoS One, 2014. 9(9): p. e107044.

271. Dong, J., et al., Promotion of bone formation using highly pure porous beta-TCP combined with bone marrow-derived osteoprogenitor cells. Biomaterials, 2002. 23(23): p. 4493-502.

272. Liu, X., et al., Co-Seeding Human Endothelial Cells with Human-Induced Pluripotent Stem CellDerived Mesenchymal Stem Cells on Calcium Phosphate Scaffold Enhances Osteogenesis and Vascularization in Rats. Tissue Eng Part A, 2017. 23(11-12): p. 546-555.

273. Matsushima, A., et al., In vivo osteogenic capability of human mesenchymal cells cultured on hydroxyapatite and on beta-tricalcium phosphate. Artif Organs, 2009. 33(6): p. 474-81.

274. Ye, X., et al., Ectopic bone regeneration by human bone marrow mononucleated cells, undifferentiated and osteogenically differentiated bone marrow mesenchymal stem cells in betatricalcium phosphate scaffolds. Tissue Eng Part C Methods, 2012. 18(7): p. 545-56.

275. Marrella, A., et al., Engineering vascularized and innervated bone biomaterials for improved skeletal tissue regeneration. Mater Today (Kidlington), 2018. 21(4): p. 362-376.

276. Eweida, A.M., et al., Enhancing mandibular bone regeneration and perfusion via axial vascularization of scaffolds. Clin Oral Investig, 2014. 18(6): p. 1671-8.

277. Laschke, M.W. and M.D. Menger, Prevascularization in tissue engineering: Current concepts and future directions. Biotechnol Adv, 2016. 34(2): p. 112-21.

278. Malhotra, A. and P. Habibovic, Calcium Phosphates and Angiogenesis: Implications and Advances for Bone Regeneration. Trends Biotechnol, 2016. 34(12): p. 983-992.

279. Albulescu, R., et al., Comprehensive In Vitro Testing of Calcium Phosphate-Based Bioceramics with Orthopedic and Dentistry Applications. Materials (Basel), 2019. 12(22).

280. Rouwkema, J. and A. Khademhosseini, Vascularization and Angiogenesis in Tissue Engineering: Beyond Creating Static Networks. Trends in Biotechnology, 2016. 34(9): p. 733-745.

281. Fuchs, S., et al., Influence of polymer content in Ca-deficient hydroxyapatite-polycaprolactone nanocomposites on the formation of microvessel-like structures. Acta Biomater, 2010. 6(8): p. 3169-77. 
282. Kang, Y., et al., Osteogenic and angiogenic potentials of monocultured and co-cultured humanbone-marrow-derived mesenchymal stem cells and human-umbilical-vein endothelial cells on threedimensional porous beta-tricalcium phosphate scaffold. Acta Biomater, 2013. 9(1): p. 4906-15.

283. Kang, Y., et al., Engineering a vascularized collagen-beta-tricalcium phosphate graft using an electrochemical approach. Acta Biomater, 2015. 11: p. 449-58.

284. Thein-Han, W. and H.H. Xu, Prevascularization of a gas-foaming macroporous calcium phosphate cement scaffold via coculture of human umbilical vein endothelial cells and osteoblasts. Tissue Eng Part A, 2013. 19(15-16): p. 1675-85.

285. Chen, W., et al., Angiogenic and osteogenic regeneration in rats via calcium phosphate scaffold and endothelial cell co-culture with human bone marrow mesenchymal stem cells (MSCs), human umbilical cord MSCs, human induced pluripotent stem cell-derived MSCs and human embryonic stem cell-derived MSCs. J Tissue Eng Regen Med, 2018. 12(1): p. 191-203.

286. Levengood, S.K., et al., Human endothelial colony forming cells undergo vasculogenesis within biphasic calcium phosphate bone tissue engineering constructs. Acta Biomater, 2011. 7(12): p. 4222-8.

287. Li, G., et al., Coculture of peripheral blood CD34+ cell and mesenchymal stem cell sheets increase the formation of bone in calvarial critical-size defects in rabbits. Br J Oral Maxillofac Surg, 2014. 52(2): p. 134-9.

288. Rivron, N.C., et al., Sonic Hedgehog-activated engineered blood vessels enhance bone tissue formation. Proc Natl Acad Sci U S A, 2012. 109(12): p. 4413-8.

289. Xu, H.H., et al., Calcium phosphate cements for bone engineering and their biological properties. Bone Res, 2017. 5: p. 17056.

290. Yu, H., et al., Improved tissue-engineered bone regeneration by endothelial cell mediated vascularization. Biomaterials, 2009. 30(4): p. 508-17.

291. Zhang, C., et al., Novel hiPSC-based tri-culture for pre-vascularization of calcium phosphate scaffold to enhance bone and vessel formation. Mater Sci Eng C Mater Biol Appl, 2017. 79: p. 296-304.

292. Zhou, J., et al., The repair of large segmental bone defects in the rabbit with vascularized tissue engineered bone. Biomaterials, 2010. 31 (6): p. 1171-9.

293. Chen, Y., et al., Promoted Angiogenesis and Osteogenesis by Dexamethasone-loaded Calcium Phosphate Nanoparticles/Collagen Composite Scaffolds with Microgroove Networks. Sci Rep, 2018. 8(1): p. 14143.

294. Klotz, B.J., et al., A Versatile Biosynthetic Hydrogel Platform for Engineering of Tissue Analogues. Adv Healthc Mater, 2019. 8(19): p. e1900979.

295. Schmittgen, T.D. and K.J. Livak, Analyzing real-time PCR data by the comparative $C(T)$ method. Nat Protoc, 2008. 3(6): p. 1101-8.

296. Doube, M., et al., BoneJ: Free and extensible bone image analysis in ImageJ. Bone, 2010. 47(6): p. 1076-9.

297. Vaquette, C., et al., Effect of culture conditions and calcium phosphate coating on ectopic bone formation. Biomaterials, 2013. 34(22): p. 5538-51.

298. Aubin, J.E., Regulation of osteoblast formation and function. Rev Endocr Metab Disord, 2001. 2(1): p. 81-94. 
299. Lenze, U., et al., Influence of osteogenic stimulation and VEGF treatment on in vivo bone formation in hMSC-seeded cancellous bone scaffolds. BMC Musculoskelet Disord, 2014. 15: p. 350. 300. Goshima, J., V.M. Goldberg, and A.I. Caplan, The origin of bone formed in composite grafts of porous calcium phosphate ceramic loaded with marrow cells. Clin Orthop Relat Res, 1991(269): p. 274-83.

301. Ben-Shaul, S., et al., Mature vessel networks in engineered tissue promote graft-host anastomosis and prevent graft thrombosis. Proc Natl Acad Sci U S A, 2019. 116(8): p. 2955-2960.

302. Mishra, R., et al., Effect of prevascularization on in vivo vascularization of poly(propylene fumarate)/ fibrin scaffolds. Biomaterials, 2016. 77: p. 255-66.

303. Di Marco, G.S., et al., Increased inorganic phosphate induces human endothelial cell apoptosis in vitro. Am J Physiol Renal Physiol, 2008. 294(6): p. F1381-7.

304. Di Marco, G.S., et al., High phosphate directly affects endothelial function by downregulating annexin II. Kidney Int, 2013. 83(2): p. 213-22.

305. Seebach, C., et al., Endothelial progenitor cells and mesenchymal stem cells seeded onto beta-TCP granules enhance early vascularization and bone healing in a critical-sized bone defect in rats. Tissue Eng Part A, 2010. 16(6): p. 1961-70.

306. Chen, X., et al., Prevascularization of a fibrin-based tissue construct accelerates the formation of functional anastomosis with host vasculature. Tissue Eng Part A, 2009. 15(6): p. 1363-71.

307. Shandalov, Y., et al., An engineered muscle flap for reconstruction of large soft tissue defects. Proc Natl Acad Sci U S A, 2014. 111(16): p. 6010-5.

308. Wang, P., et al., Stem Cells and Calcium Phosphate Cement Scaffolds for Bone Regeneration. J Dent Res, 2014. 93(7): p. 618-25.

309.Zhou, X., et al., Phosphate/pyrophosphate and MV-related proteins in mineralisation: discoveries from mouse models. Int J Biol Sci, 2012. 8(6): p. 778-90.

310. Diez-Escudero, A., et al., In vitro degradation of calcium phosphates: Effect of multiscale porosity, textural properties and composition. Acta Biomater, 2017. 60: p. 81-92.

311. Samavedi, S., A.R. Whittington, and A.S. Goldstein, Calcium phosphate ceramics in bone tissue engineering: a review of properties and their influence on cell behavior. Acta Biomater, 2013. 9(9): p. 8037-45.

312. Basu, P., et al., Calcium Phosphate Incorporated Bacterial Cellulose-Polyvinylpyrrolidone Based Hydrogel Scaffold: Structural Property and Cell Viability Study for Bone Regeneration Application. Polymers (Basel), 2019. 11(11).

313. Moccia, F., R. Berra-Romani, and F. Tanzi, Update on vascular endothelial $\mathrm{Ca}(2+)$ signalling: A tale of ion channels, pumps and transporters. World J Biol Chem, 2012. 3(7): p. 127-58.

314. Pinton, P., et al., Calcium and apoptosis: ER-mitochondria Ca2+ transfer in the control of apoptosis. Oncogene, 2008. 27(50): p. 6407-18.

315. Shroff, R., D.A. Long, and C. Shanahan, Mechanistic insights into vascular calcification in CKD. J Am Soc Nephrol, 2013. 24(2): p. 179-89.

316. Askar, A.M., Hyperphosphatemia. The hidden killer in chronic kidney disease. Saudi Med J, 2015. 36(1): p. 13-9. 
317. Giachelli, C.M., Vascular calcification: in vitro evidence for the role of inorganic phosphate. J Am Soc Nephrol, 2003. 14(9 Suppl 4): p. S300-4.

318. Zhang, Y., et al., Dissolution properties of different compositions of biphasic calcium phosphate bimodal porous ceramics following immersion in simulated body fluid solution. Ceramics International, 2013. 39(6): p. 6751-6762.

319. Persy, V. and P. D'Haese, Vascular calcification and bone disease: the calcification paradox. Trends Mol Med, 2009. 15(9): p. 405-16.

320. Chapman, D.M., et al., Subcutaneous microvascular (capillary) calcification. Another basis for livedo-like skin changes? Clin Exp Dermatol, 1995. 20(3): p. 213-7.

321.Peng, A., et al., Adverse effects of simulated hyper- and hypo-phosphatemia on endothelial cell function and viability. PLoS One, 2011. 6(8): p. e23268.

322. Takeda, E., et al., A novel function of phosphate-mediated intracellular signal transduction pathways. Adv Enzyme Regul, 2006. 46: p. 154-61.

323. Yao, Y., et al., A role for the endothelium in vascular calcification. Circ Res, 2013. 113(5): p. 495-504.

324. Orriss, I.R., S.E. Taylor, and T.R. Arnett, Rat osteoblast cultures. Methods Mol Biol, 2012. 816: p. 31-41.

325. Azpiazu, D., et al., Role of pyrophosphate in vascular calcification in chronic kidney disease. Nefrología (English Edition), 2018. 38(3): p. 250-257.

326. Inden, M., et al., Localization of type-III sodium-dependent phosphate transporter 2 in the mouse brain. Brain Res, 2013. 1531: p. 75-83.

327. Villa-Bellosta, R. and V. Sorribas, Phosphonoformic acid prevents vascular smooth muscle cell calcification by inhibiting calcium-phosphate deposition. Arterioscler Thromb Vasc Biol, 2009. 29(5): p. 761-6.

328. Yoshiko, Y., et al., Osteoblast autonomous Pi regulation via Pit1 plays a role in bone mineralization. Mol Cell Biol, 2007. 27(12): p. 4465-74.

329. Moffat, K.L., et al., Engineering Functional Tissues, in Principles of Tissue Engineering. 2014. p. 237-259.

330. Butler, D.L., S.A. Goldstein, and F. Guilak, Functional tissue engineering: the role of biomechanics. J Biomech Eng, 2000. 122(6): p. 570-5.

331. Guilak, F., et al., Biomechanics and mechanobiology in functional tissue engineering. J Biomech, 2014. 47(9): p. 1933-40.

332. Driessen, N.J., et al., Remodelling of the angular collagen fiber distribution in cardiovascular tissues. Biomech Model Mechanobiol, 2008. 7(2): p. 93-103.

333. Schriefl, A.J., et al., Determination of the layer-specific distributed collagen fibre orientations in human thoracic and abdominal aortas and common iliac arteries. J R Soc Interface, 2012. 9(71): p. 1275-86.

334. de Valence, S., et al., Advantages of bilayered vascular grafts for surgical applicability and tissue regeneration. Acta Biomater, 2012. 8(11): p. 3914-20.

335. Zhang, Z., et al., Pore size, tissue ingrowth, and endothelialization of small-diameter microporous polyurethane vascular prostheses. Biomaterials, 2004. 25(1): p. 177-187.

336. Muylaert, D.E., et al., Early in-situ cellularization of a supramolecular vascular graft is modified by synthetic stromal cell-derived factor-1alpha derived peptides. Biomaterials, 2016. 76: p. 187-95. 
337. Scott, D., et al., High pulsatility flow stimulates smooth muscle cell hypertrophy and contractile protein expression. Am J Physiol Lung Cell Mol Physiol, 2013. 304(1): p. L70-81.

338. Zhao, S., et al., Synergistic effects of fluid shear stress and cyclic circumferential stretch on vascular endothelial cell morphology and cytoskeleton. Arterioscler Thromb Vasc Biol, 1995. 15(10): p. 1781-6.

339. Greenwald, S.E. and C.L. Berry, Improving vascular grafts: the importance of mechanical and haemodynamic properties. J Pathol, 2000. 190(3): p. 292-9.

340. Hochleitner, G., et al., Melt Electrowriting of Thermoplastic Elastomers. Macromol Rapid Commun, 2018. 39(10): p. e1800055.

341. Traore, M.A. and S.C. George, Tissue Engineering the Vascular Tree. Tissue Eng Part B Rev, 2017. 23(6): p. 505-514.

342. Patel, A., et al., Elastin biosynthesis: The missing link in tissue-engineered blood vessels. Cardiovasc Res, 2006. 71(1): p. 40-9.

343. Wise, S.G., et al., A multilayered synthetic human elastin/polycaprolactone hybrid vascular graft with tailored mechanical properties. Acta Biomater, 2011. 7(1): p. 295-303.

344. Landau, S., et al., Tropoelastin coated PLLA-PLGA scaffolds promote vascular network formation. Biomaterials, 2017. 122: p. 72-82.

345. Xu, J. and G.P. Shi, Vascular wall extracellular matrix proteins and vascular diseases. Biochim Biophys Acta, 2014. 1842(11): p. 2106-2119.

346. Dahl, S.L., et al., Readily available tissue-engineered vascular grafts. Sci Transl Med, 2011. 3(68): p. 68 ra9.

347. Kirkton, R.D., et al., Bioengineered human acellular vessels recellularize and evolve into living blood vessels after human implantation. Sci Transl Med, 2019. 11 (485).

348. Syedain, Z.H., et al., A completely biological "off-the-shelf" arteriovenous graft that recellularizes in baboons. Sci Transl Med, 2017. 9(414).

349. Choi, M.L., et al., Increased patency of artificial microvascular grafts using arteriovenous fistula loops: a two-stage procedure for lengthening the pedicle of free-tissue transfer. J Reconstr Microsurg, 1996. 12(5): p. 283-90.

350. Taub, P.J., et al., Staging arteriovenous fistula loops for lengthening of free-flap pedicles. J Reconstr Microsurg, 1999. 15(2): p. 123-5.

351. Asano, Y., et al., Sprouting from arteriovenous shunt vessels with increased blood flow. Med Biol Eng Comput, 2005. 43(1): p. 126-30.

352. Polykandriotis, E., et al., Intrinsic versus extrinsic vascularization in tissue engineering. Adv Exp Med Biol, 2006. 585: p. 311-26.

353. Polykandriotis, E., et al., The venous graft as an effector of early angiogenesis in a fibrin matrix. Microvasc Res, 2008. 75(1): p. 25-33.

354. Painter, P.R., P. Eden, and H.U. Bengtsson, Pulsatile blood flow, shear force, energy dissipation and Murray's Law. Theor Biol Med Model, 2006. 3: p. 31.

355. Kwon, H.M., et al., Adventitial vasa vasorum in balloon-injured coronary arteries. Journal of the American College of Cardiology, 1998. 32(7): p. 2072-2079. 
356. Mulligan-Kehoe, M.J. and M. Simons, Vasa vasorum in normal and diseased arteries. Circulation, 2014. 129(24): p. 2557-66.

357. Pennel, T., et al., Transmural capillary ingrowth is essential for confluent vascular graft healing. Acta Biomater, 2018. 65: p. 237-247.

358. Webber, M.J., et al., A perspective on the clinical translation of scaffolds for tissue engineering. Ann Biomed Eng, 2015. 43(3): p. 641-56.

359. de Valence, S., et al., Long term performance of polycaprolactone vascular grafts in a rat abdominal aorta replacement model. Biomaterials, 2012. 33(1): p. 38-47.

360. Abbott, W.M., et al., Effect of compliance mismatch on vascular graft patency. Journal of Vascular Surgery, 1987. 5(2): p. 376-382.

361. van der Stok, J., et al., Full regeneration of segmental bone defects using porous titanium implants loaded with BMP-2 containing fibrin gels. Eur Cell Mater, 2015. 29: p. 141-53; discussion 153-4.

362. Keane, T.J. and S.F. Badylak, The host response to allogeneic and xenogeneic biological scaffold materials. J Tissue Eng Regen Med, 2015. 9(5): p. 504-11.

363. Bartneck, M., et al., Inducing healing-like human primary macrophage phenotypes by 3D hydrogel coated nanofibres. Biomaterials, 2012. 33(16): p. 4136-46.

364. Brown, B.N. and S.F. Badylak, Biocompatibility and Immune Response to Biomaterials, in Regenerative Medicine Applications in Organ Transplantation. 2014. p. 151-162.

365. Brown, B.N., et al., Macrophage polarization: an opportunity for improved outcomes in biomaterials and regenerative medicine. Biomaterials, 2012. 33(15): p. 3792-802.

366. Anderson, J.M., A. Rodriguez, and D.T. Chang, Foreign body reaction to biomaterials. Semin Immunol, 2008. 20(2): p. 86-100.

367. Simionescu, A., et al., Inflammation in cardiovascular tissue engineering: the challenge to a promise: a minireview. Int J Inflam, 2011. 2011: p. 958247.

368. Goonoo, N., Modulating Immunological Responses of Electrospun Fibers for Tissue Engineering. Advanced Biosystems, 2017. 1(8).

369. Wissing, T.B., et al., Biomaterial-driven in situ cardiovascular tissue engineering-a multi-disciplinary perspective. NPJ Regen Med, 2017. 2: p. 18.

370. van Loon, S., et al., The Immune Response in In Situ Tissue Engineering of Aortic Heart Valves. 2013. p. 207-245.

371. Chung, L., et al., Key players in the immune response to biomaterial scaffolds for regenerative medicine. Adv Drug Deliv Rev, 2017. 114: p. 184-192.

372. Hoffman, T., A. Khademhosseini, and R. Langer, Chasing the Paradigm: Clinical Translation of 25 Years of Tissue Engineering. Tissue Eng Part A, 2019. 25(9-10): p. 679-687.

373. Dlaska, C.E., et al., Clinical Translation in Tissue Engineering-The Surgeon's View. Current Molecular Biology Reports, 2015. 1(2): p. 61-70.

374. Kokosis, G., et al., Mandibular Reconstruction Using the Free Vascularized Fibula Graft: An Overview of Different Modifications. Arch Plast Surg, 2016. 43(1): p. 3-9.

375. Rana, M., et al., Reconstruction of mandibular defects - clinical retrospective research over a 10-year period. Head Neck Oncol, 2011. 3: p. 23. 
376. Hartman, E.H., P.H. Spauwen, and J.A. Jansen, Donor-site complications in vascularized bone flap surgery. J Invest Surg, 2002. 15(4): p. 185-97.

377. Hayden, R.E., D.P. Mullin, and A.K. Patel, Reconstruction of the segmental mandibular defect: current state of the art. Curr Opin Otolaryngol Head Neck Surg, 2012. 20(4): p. 231-6.

378. Zimmermann, C.E., et al., Donor site morbidity after microvascular fibula transfer. Clin Oral Investig, 2001. 5(4): p. 214-9.

379. Weigand, A., et al., The Arteriovenous Loop: Engineering of Axially Vascularized Tissue. Eur Surg Res, 2018. 59(3-4): p. 286-299.

380. Jiang, F., et al., Neovascularization in an arterio-venous loop-containing tissue engineering chamber: role of NADPH oxidase. J Cell Mol Med, 2008. 12(5B): p. 2062-72.

381. Lokmic, Z., et al., An arteriovenous loop in a protected space generates a permanent, highly vascular, tissue-engineered construct. FASEB J, 2007. 21(2): p. 511-22.

382. Yuan, Q., et al., PHDs inhibitor DMOG promotes the vascularization process in the AV loop by HIF-1a up-regulation and the preliminary discussion on its kinetics in rat. BMC Biotechnol, 2014. 14: p. 112.

383. Schmidt, V.J., et al., High flow conditions increase connexin43 expression in a rat arteriovenous and angioinductive loop model. PLoS One, 2013. 8(11): p. e78782.

384. Erol, O.O. and M. Sira, New capillary bed formation with a surgically constructed arteriovenous fistula. Plast Reconstr Surg, 1980. 66(1): p. 109-15.

385. Hofer, S.O., et al., Increasing the volume of vascularized tissue formation in engineered constructs: an experimental study in rats. Plast Reconstr Surg, 2003. 111(3): p. 1186-92; discussion 1193-4.

386. Cassell, O.C., et al., The influence of extracellular matrix on the generation of vascularized, engineered, transplantable tissue. Ann NY Acad Sci, 2001. 944: p. 429-42.

387. Mian, R., et al., Formation of new tissue from an arteriovenous loop in the absence of added extracellular matrix. Tissue Eng, 2000. 6(6): p. 595-603.

388. Tanaka, Y., et al., Generation of an autologous tissue (matrix) flap by combining an arteriovenous shunt loop with artificial skin in rats: preliminary report. Br J Plast Surg, 2000. 53(1): p. 51-7.

389. Fiegel, H.C., et al., Foetal hepatocyte transplantation in a vascularized AV-Loop transplantation model in the rat. J Cell Mol Med, 2010. 14(1-2): p. 267-74.

390. Choi, Y.S., et al., Engineering cardiac tissue in vivo from human adipose-derived stem cells. Biomaterials, 2010. 31(8): p. 2236-42.

391. Messina, A., et al., Generation of a vascularized organoid using skeletal muscle as the inductive source. FASEB J, 2005. 19(11): p. 1570-2.

392. Robering, J.W., et al., Mesenchymal stem cells promote lymphangiogenic properties of lymphatic endothelial cells. J Cell Mol Med, 2018.

393. Arkudas, A., et al., Pedicled Transplantation of Axially Vascularized Bone Constructs in a Critical Size Femoral Defect. Tissue Eng Part A, 2018. 24(5-6): p. 479-492.

394. Boos, A.M., et al., Engineering axially vascularized bone in the sheep arteriovenous-loop model. J Tissue Eng Regen Med, 2013. 7(8): p. 654-64. 
395. Arkudas, A., et al., Automatic quantitative micro-computed tomography evaluation of angiogenesis in an axially vascularized tissue-engineered bone construct. Tissue Eng Part C Methods, 2010. 16(6): p. 1503-14.

396. Wu, X., et al., The effects of different vascular carrier patterns on the angiogenesis and osteogenesis of BMSC-TCP-based tissue-engineered bone in beagle dogs. J Tissue Eng Regen Med, 2017. 11(2): p. 542-552.

397.Dong, Q.S., et al., Prefabrication of axial vascularized tissue engineering coral bone by an arteriovenous loop: a better model. Mater Sci Eng C Mater Biol Appl, 2012. 32(6): p. 1536-41.

398. Warnke, P.H., et al., Growth and transplantation of a custom vascularised bone graft in a man. The Lancet, 2004. 364(9436): p. 766-770.

399. Beier, J.P., et al., De novo generation of an axially vascularized processed bovine cancellous-bone substitute in the sheep arteriovenous-loop model. Eur Surg Res, 2011. 46(3): p. 148-55.

400. Bitto, F.F., et al., Myogenic differentiation of mesenchymal stem cells in a newly developed neurotised AV-loop model. Biomed Res Int, 2013. 2013: p. 935046.

401. Jones, E.A., F. le Noble, and A. Eichmann, What determines blood vessel structure? Genetic prespecification vs. hemodynamics. Physiology (Bethesda), 2006. 21: p. 388-95.

402. Buschmann, I. and W. Schaper, Arteriogenesis Versus Angiogenesis: Two Mechanisms of Vessel Growth. News Physiol Sci, 1999. 14: p. 121-125.

403. Heil, M., et al., Arteriogenesis versus angiogenesis: similarities and differences. J Cell Mol Med, 2006. 10(1): p. 45-55.

404. Bruzauskaite, I., et al., Scaffolds and cells for tissue regeneration: different scaffold pore sizesdifferent cell effects. Cytotechnology, 2016. 68(3): p. 355-69.

405. Pennel, T., P. Zilla, and D. Bezuidenhout, Differentiating transmural from transanastomotic prosthetic graft endothelialization through an isolation loop-graft model. J Vasc Surg, 2013. 58(4): p. 1053-61.

406. Sanchez, P.F., E.M. Brey, and J.C. Briceno, Endothelialization mechanisms in vascular grafts. JTissue Eng Regen Med, 2018. 12(11): p. 2164-2178.

407. White, R.A., The effect of porosity and biomaterial on the healing and long-term mechanical properties of vascular prostheses. ASAIO Trans, 1988. 34(2): p. 95-100.

408. Clowes, A.W., T.R. Kirkman, and M.A. Reidy, Mechanisms of arterial graft healing. Rapid transmural capillary ingrowth provides a source of intimal endothelium and smooth muscle in porous PTFE prostheses. Am J Pathol, 1986. 123(2): p. 220-30.

409. Zhong, S., Y. Zhang, and C.T. Lim, Fabrication of large pores in electrospun nanofibrous scaffolds for cellular infiltration: a review. Tissue Eng Part B Rev, 2012. 18(2): p. 77-87.

410. Lee, B.L., et al., Femtosecond laser ablation enhances cell infiltration into three-dimensional electrospun scaffolds. Acta Biomater, 2012. 8(7): p. 2648-58.

411. Lim, Y.C., et al., Micropatterning and characterization of electrospun poly(epsilon-caprolactone)/ gelatin nanofiber tissue scaffolds by femtosecond laser ablation for tissue engineering applications. Biotechnol Bioeng, 2011. 108(1): p. 116-26.

412. See-Tho, K. and E.J. Harris, Jr., Thrombosis with outflow obstruction delays thrombolysis and results in chronic wall thickening of rat veins. J Vasc Surg, 1998. 28(1): p. 115-22; discussion 123. 
413. Galie, P.A., et al., Fluid shear stress threshold regulates angiogenic sprouting. Proc Natl Acad Sci U S A, 2014. 111 (22): p. 7968-73.

414. Dvorak, H.F., et al., Vascular permeability factor/vascular endothelial growth factor, microvascular hyperpermeability, and angiogenesis. Am J Pathol, 1995. 146(5): p. 1029-39. 

Nederlandse samenvatting

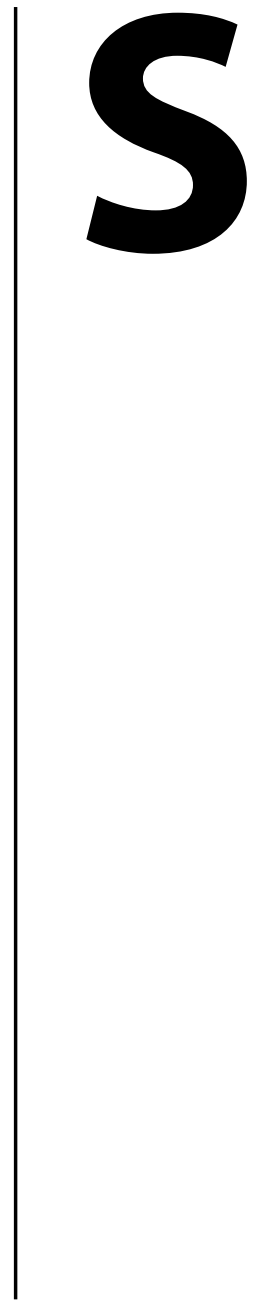




\section{Nederlandse samenvatting}

Het menselijk lichaam heeft het vermogen om zichzelf te herstellen als er bijvoorbeeld een wond of breuk is. Indien het defect echter een dusdanig grote afmeting heeft, of als het weefsel teveel beschadigd is, kan dit niet altijd meer door het lichaam zelf worden genezen. Dit kan het geval zijn bij grote botdefecten na bijvoorbeeld een ongeval, door aangeboren afwijkingen of na het verwijderen van een tumor. Zulke aandoeningen kunnen ook in het aangezicht voorkomen, bijvoorbeeld in de onderkaak. De huidige therapieën voor de genezing van dergelijke defecten zijn gericht op het vervangen van het zieke of ontbrekende botweefsel. Dit kan, onder andere, door metalen implantaten of door botweefsel dat wordt verkregen uit een ander gedeelte van het lichaam, bijvoorbeeld het onderbeen of onderarm in het geval van een groot kaakdefect. Het nadeel van deze toepassingen is dat een metalen implantaat niet als lichaamseigen wordt gezien en het defect niet geneest. Het zal na een tijd weer vervangen moet worden. Aan de andere kant maak je bij het gebruik van weefseltransplantaties een nieuw defect in het lichaam, hetgeen lokaal weer tot ongemakken en complicaties kan zorgen. Daarnaast is er niet altijd genoeg botweefsel beschikbaar.

Het veld van de regeneratieve geneeskunde richt zich op het vinden van nieuwe oplossingen door biologische implantaten in het laboratorium te ontwikkelen. Deze kunnen het beschadigde weefsel vervangen of het lichaam aansturen om het weefsel zelf te genezen. Dergelijke implantaten, zogenoemde constructen, kunnen gemaakt worden door middel van combinaties van signalerende factoren, stamcellen (van de patiënt zelf of van donoren) en/of ondersteunde dragermaterialen, zogenaamde scaffolds. Deze constructen kunnen vooraf in het laboratorium gekweekt worden, of direct na het maken in het lichaam van de patiënt geïmplanteerd worden. Dit proefschrift focust op het maken van gekweekt botweefsel vanuit lichaamseigen cellen die uiteindelijk zou kunnen dienen als een implantaat voor patiënten met een botdefect, bijvoorbeeld in de onderkaak.

Het grootste knelpunt bij de ontwikkeling van grote weefseltransplantaten is het in leven houden van de cellen in het gekweekte construct. In het lichaam worden cellen in leven gehouden door de bloedstroom. Deze transporteert zuurstof en voedingsstoffen naar de cellen en voert afvalstoffen af. Zonder toevoer van deze benodigdheden zullen de cellen in het lichaam, of in een botconstruct in het laboratorium, sterven en hun functie verliezen. Om dit probleem tegen te gaan, kan er in het laboratorium een netwerk van bloedvaten in het botconstruct gecreëerd worden, hoofdstuk 1 van dit proefschrift dient als een inleiding over dit onderwerp. Het stimuleren van de vaatgroei in botconstructen zal meerdere voordelen met zich meebrengen: het zal de afsterving van het weefsel in het laboratorium tegengaan, en bovendien kunnen de gekweekte bloedvaten direct aan de bloedvaten van de patiënt gehecht worden voor een onmiddellijke bloeddoorstroming 
na implanteren. Om dit zo effectief mogelijk te laten verlopen, is het van belang om het bloedvatnetwerk zo natuurgetrouw mogelijk na te maken.

Het bloedvatnetwerk in het lichaam is te vinden in de vorm van een vasculaire boom. Dit houdt in dat er grote vaten met dikke wanden uit het hart komen, welke daarna aftakken naar kleinere vaten die het orgaan ingaan waar ze vervolgens uit lopen in de kleinste haarvaten (capillairen) om de individuele cellen van de voedingsstoffen voorzien. Voor het maken van een levend botweefsel is het dus van belang dat we én de grote bloedvaten (macro-vaten) nabootsen die dienen als een verbindingselement om het geheel aan de patiënt te hechten, én de capillairen (micro-vaten) die vervolgens het bloed direct naar de botcellen vervoeren. De uitdaging in de toekomst is het uiteindelijk aan elkaar verbinden van deze verschillende niveaus van bloedvaten, samen met het tot stand brengen van een bloedstroom in het construct (op het laboratorium).

Naast het creëren van de verschillende niveaus van bloedvaten is het ook van belang om de specifieke architectuur van de desbetreffende vaten na te maken. Het zo goed mogelijk nabootsen van deze kleine details zal de uiteindelijke functionaliteit van het construct ten goede komen. Het stelt het construct bijvoorbeeld in staat om de krachten die het lichaam erop uitoefent te weerstaan, bijvoorbeeld de bloeddruk in bloedvaten.

Kijkende naar de specifieke architectuur van de grote bloedvaten valt het op dat er verschillende weefsellagen aanwezig zijn, waarin elke laag weer zijn eigen functie heeft. De binnenste laag (tunica intima) verwijst naar een enkele laag van'endotheelcellen' die de binnenkant van het vat bekleedt. Deze cellen zorgen dat er geen onnodige bloedstolling optreedt en ze reageren op de snelheid van de bloedstroom, waarbij ze stoffen produceren die het passerende bloedvolume kunnen reguleren. De regulatie daarvan vindt plaats doordat gladde spiercellen in de omringende weefsel laag (tunica media) reageren op de signaalstoffen die deze endotheelcellen uitscheiden. De spiercellen reageren hierop door samen te trekken of juist te ontspannen. Als gevolg hiervan zal het bloedvat wijder of juist smaller worden en zal de bloeddruk veranderen.

Voor het uitoefenen van deze specifieke functie moeten de gladde spiercellen in een specifieke circulaire oriëntatie liggen, en is het van belang dat ze daadwerkelijk het vermogen hebben om te kunnen samentrekken. In hoofdstuk 2 is er gekeken naar de ontwikkeling van een nieuwe combinatie van 3D print technieken (electrospinnen) om een scaffold structuur te maken die de 2 weefsellagen natuurgetrouw nabootst. Vervolgens is er in hoofdstuk 3 een mechanische opstelling ontwikkeld die de bloedstroom simuleert. De omgeving die het construct na implantatie zal tegenkomen, wordt daardoor in het laboratorium beter nagebootst. In beide hoofdstukken is er onderzocht of de specifieke scaffold structuur, en de simulerende omgeving, de twee gebruikte typen stamcellen kunnen sturen om functionele endotheelcellen of functionele gladde spiercellen te vormen. 
De grote bloedvaten zullen uiteindelijk aftakken in kleine haarvaten die eindigen bij de botcellen. Het maken van dit gedeelte van het construct is uitgelegd in hoofdstuk 4, waarin dezelfde twee typen stamcellen gecombineerd worden in een fibreus materiaal met hoog watergehalte (hydrogel). Deze gel creëert samen met specifieke signaalstoffen een omgeving waarin het ene celtype een netwerk vormt van haarvaten, terwijl tegelijkertijd het andere type stamcel aangezet wordt om te veranderen naar cellen die steun geven aan de bloedvaten, en ook naar botachtige cellen. In hoofdstuk 5 is vervolgens een manier gevonden om deze hydrogelconstructen uit te breiden met een scaffold materiaal dat het mineraal van het bot nabootst. Hierdoor krijgen de cellen meer botvormende signalen en wordt het construct stabieler waardoor het beter geïmplanteerd kan worden. Dit veelvuldig gebruikte mineraal bleek echter een negatieve invloed te hebben op de ontwikkeling van het vaatnetwerk. In hoofdstuk 6 hebben wij aangetoond dat een verhoogde toevoeging van dit mineraal, die botvorming stimuleert, juist een negatieve invloed had op het behouden van een bloedvatnetwerk onder kweekomstandigheden. Vervolgonderzoeken worden momenteel uitgevoerd om te bestuderen of dit probleem opgelost kan worden.

Tevens is er in dit proefschrift achtergrondinformatie te vinden over toekomstige experimentele opzetten die het uiteindelijke koppelen van de verschillende vaatniveaus van de vasculaire boom in het laboratorium (appendix 2) en na implantatie (appendix 1) in één construct zouden kunnen bewerkstelligen.

\section{Toekomstperspectief en conclusie}

Het in leven houden van cellen is een belangrijk knelpunt bij het maken van levend botweefsel op het laboratorium en na implantatie. Het toevoegen van bloedvaten aan een dergelijk construct kan als oplossing dienen. Om ervoor te zorgen dat de vaten en botcellen van een bloedtoevoer voorzien kunnen worden, zijn er verschillende niveaus van bloedvaten nodig; van grote vaten die gehecht kunnen worden aan die van de patiënt tot kleine haarvaten die naar de botcellen groeien. In deze thesis hebben we scaffold structuren gemaakt voor grote vaten door middel van een nieuwe combinatie van 3D print technieken (electrospinnen). Vervolgens hebben we een netwerk van kleine haarvaten gemaakt in een hydrogel, samen met stamcellen die zich tot botcellen ontwikkelen en mineralen die het bot nabootsen. Door het samenbrengen van deze bloedvaten zou er uiteindelijk een stuk botweefsel gevormd kunnen worden met daarin een netwerk van bloedvaten in de vorm van een vasculaire boom. Deze structuur zou een directe bloedtoevoer kunnen bewerkstelligen in het laboratorium en na implantatie. Dit ontwerp zou de nadelen van de huidige behandel methodes (metalen implantaten of weefseltransplantaties) ten boven kunnen komen, en kan in de toekomst dienen als gepersonaliseerd transplantatiemateriaal voor patiënten met een groot botdefect. 

List of publications

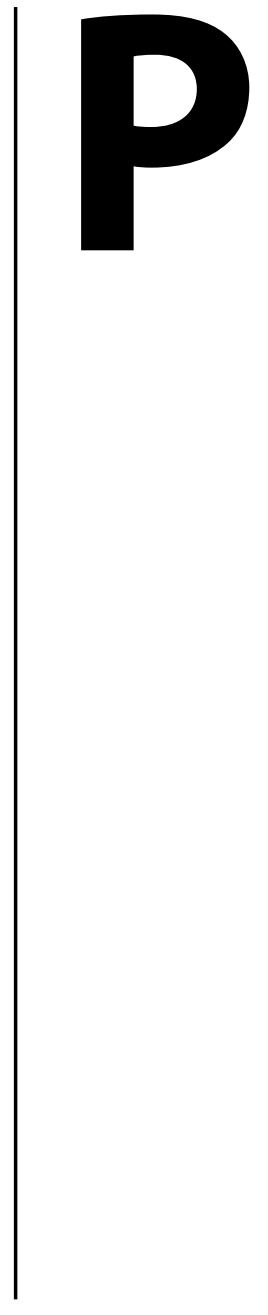




\section{List of publications}

1. B.J. Klotz, K.S. Lim, Y.X. Chang, B.G. Soliman, I. Pennings, F.P.W. Melchels, T.B.F. Woodfield, A.J.W.P. Rosenberg, J. Malda, D. Gawlitta. Engineering of a complex bone tissue model with endothelialised channels and capillary-like networks. European Cells and Materials, 2018. 30(35).

2. I. Pennings, L.A. van Dijk, J. van Huuksloot, J.O. Fledderus, K. Schepers, A.K. Braat, E.C. Hsiao, E. Barruet, B.M. Morales, M.C. Verhaar, A.J.W.P. Rosenberg, D. Gawlitta. Effect of donor variation on osteogenesis and vasculogenesis in hydrogel co-cultures. Journal of Tissue Engineering and Regenerative Medicine. 2019. 13(3).

3. I. Pennings*, T. Jungst*, M. Schmitz, A.J.W.P. Rosenberg, J. Groll\#, D. Gawlitta\#. Heterotypic scaffold design orchestrates primary cell organization and phenotypes in cocultured small diameter vascular grafts. Advanced Functional Materials, 2019. 29(43).

4. I. Pennings*, E.E. van Haaften*, T. Jungst, J.A. Bulsink, A.J.W.P Rosenberg, J. Groll, C.V.C. Bouten, N.A. Kurniawan, A.I.P.M. Smits, D. Gawlitta. Layer-specific cell differentiation in bi-layered vascular grafts under flow perfusion. Biofabrication, 2019. 12(1).

5. I. Pennings, A. Longoni, Q. Vallmajo-Martin, C. Cheng, M. Ehrbar, A.J.W.P Rosenberg, D. Gawlitta. In vitro pre-differentiation of pre-vascularized bone constructs containing calcium phosphates accelerates the onset of in vivo bone formation. Submitted.

6. A. Longoni, I. Pennings, M. Cuenca Lopera, M.H.P. van Rijen, V. Peperzak, A.J.W.P. Rosenberg, R. Levato, D. Gawlitta. Endochondral bone regeneration by non-autologous mesenchymal stem cells. Frontiers in Bioengineering and Biotechnology, 2020.

7. I. Pennings, C. Cheng, A.J.W.P. Rosenberg, D. Gawlitta. Osteogenic stimulation triggers microvascular regression in the pre-vascularization strategy for bone tissue vascularization. In preparation.

8. A. Longoni, I. Pennings, G.C.J. Lindberg, T.B.F. Woodfield, A.J.W.P. Rosenberg, K.S. Lim, D. Gawlitta. Vitreous humor as instructive biomaterial to support mesenchymal stem cell hypertrophy and endochondral ossification. In preparation.

*,\# Authors contributed equally 



\section{Dankwoord}

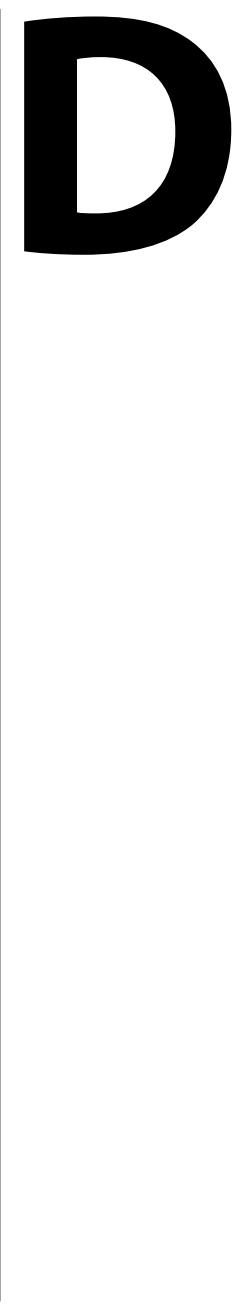




\section{Dankwoord}

Dan eindelijk het stuk van de thesis wat het meest gelezen zal gaan worden (of als enige), en wellicht ook een van de belangrijkste stukken. Want zonder jullie hulp en steun had ik dit proefschrift niet op deze manier af kunnen ronden. $\mathrm{Nu}$ is het tijd om jullie even uitgebreid (want ik hou van langdradig, en nu mag het eindelijk) in het zonnetje te zetten:

\section{Mijn promotieteam}

\section{Team kaak}

Mijn promotor van het kaak team, Prof. dr. Rosenberg. Beste Toine, bedankt voor de oneindige interesse in het onderzoek en het enthousiasme over de resultaten die behaald werden. Het is fijn om te zien dat de resultaten, waar ik vaak zelf erg kritisch over was, positief ontvangen worden door een clinicus die er ook een toepassing van inziet. Bedankt voor de hulp met de microchirurgie, de waardering en de oprechte interesse in de persoon achter het onderzoek. Met hard werken is het uiteindelijk toch gelukt om dit proefschrift op tijd af te krijgen, want Toine, een deadline is een deadline...

Mijn co-promotor, dr. ir. Gawlitta. Beste Debby, we hebben elkaar 7 jaar geleden leren kennen tijdens mijn bachelor stage. Vanuit daar ben terecht gekomen in mijn masterstage en ben uiteindelijk ook het PhD traject met jou aangegaan, waarbij er een mooi meidenteam (best lady's team) is gevormd. Hoe gestructureerd ik onze meetings ook in probeerde te gaan, het liefst met liters koffie, eindigde we toch vaak met anekdotes over het weekend of (salsa)feesten, en de laatste twee jaar vaak ook over je mooie dochters. Naast de begeleiding op het lab heb je ook echt een hart gehad voor mijn persoonlijke ontwikkeling. Hiervoor heb je me de kansen gegeven om te reizen naar San-Francisco, Würzburg en Erlangen, en hebt mij de vrijheid geven om mijn project geheel op te zetten zoals ik dat wilde. Hoe frustrerend het daarnaast was om stukken volledig rood teruggestuurd te krijgen (mieren...), het geeft wel weer hoeveel energie en tijd je in ons werk steekt. Daarnaast heeft het er altijd toe geleid dat er een mooi stuk (pareltje) uit kwam, en een sticker! Samen gezellig op congres naar Maastricht, Rhodos en Würzburg, waarbij vooral het gala van de laatste me nog vers in het geheugen staat.... En ik blijf erbij Debby, jouw verhalen zijn het beste anticonceptie middel ooit.

\section{Team nefrologie/vascular}

Mijn promotor, Prof. dr. Verhaar, beste Marianne, ook bij jou heb ik je oprechte interesse in mij als persoon zeer gewaardeerd, en heb genoten van je verbazing over mijn 'niet regeldrang' als ik op reis ga. Beste dr. Cheng, Caroline, bedankt voor de kritische input tijdens de vascular meetings, en de hoognodige steun en input in de laatste fase van mijn $\mathrm{PhD}$, waarbij er op de valreep nog een mooi hoofdstuk bij is gekomen. 
Hooggeleerde en zeergeleerde leden, bedankt voor de kans om mijn $\mathrm{PhD}$ te voltooien in deze commissie!

Beste beoordelingscommissie, bedankt voor het plaatsnemen in de commissie en voor jullie tijd om mijn proefschrift te beoordelen.

\section{Mijn collega's/my collegues}

Alessia, where to start... My favorite crazy Italian, my social butterfly and my dear paranimf. Even though we started off as colleagues, our professional relationship quickly grew into a very close friendship. The endless amount of time we spent together working at the lab, talking and shouting in the (flex)office, and doing surgery (never again). We shared even more precious moments spending our holidays together, eating dinner, working out in the gym (I finally managed!), and shooting zombies. Not to mention an endless resource of your delicious homemade meals, wine and Ben\&Jerry's. That last combination re-appeared more than once in the last couple of years, as it became our magical recipe to overcome the obstacles we faced, and to celebrate our successes. I knew I could always count on you to help me on the lab and that you were just one text away from me at home.

How did we manage to work that well together? It will remain a mystery... Sometimes we are like day and night. On the one hand you have a chaotic, messy and loud Italian, while on the other hand you have an organized, practical and down to earth Dutchy. We were asking for trouble! 'Alessia, if you don't clean up now, I will throw away all of your stuff', 'Ale, how do you even survive?' and 'This is NOT where it is supposed to be!' But, our magical combination created some unforgettable years filled with fun and laughter. An incredible friendship I will always cherish. Ale, my dear crazy Italian: grazie mille.

Geachte dr. Utomo, lieve Lizette. 'Mag ik even ventileren?' Dankjewel voor je praktische tips en tricks, je goed doordachte antwoorden en dat je altijd voor me klaar stond ongeacht hoe druk je het zelf had. Het is ongelofelijk hoe gedisciplineerd je bent en hoeveel werk je kan verzetten. En even tussendoor: bizar hoeveel kennis iemand kan hebben over wijn en whisky.... Je bent een vrouw met vele talenten, and yes I am getting to the point: dankjewel lieve Lizette, zorg goed voor jezelf en kies de weg die jou gelukkig maakt.

Lieve dr. Klotz, onze Barbi. Samen met Alessia vormde we een mooi meiden team. Wat heb ik jouw kalmte en nuchtere kijk op het leven kunnen waarderen in de stressvolle tijden. Al kon ik ook altijd wel lachen om momenten wanneer ook jij geïrriteerd het kantoor binnen kwam stampen omdat je experiment niet 'perfect' uitgekomen was, of omdat je vond dat onderzoek toch allemaal maar onzin was. Naast de steun van jou in ons vascularisatie project, heb ik zeer genoten van de vele (speciaal) biertjes die we tijdens de 
borrels vakkundig hebben weggewerkt. En als ik met jou op pad was, leek het altijd alsof mijn richtingsgevoel piekfijn in orde was. Danke schön liebe Barbi.

Joao, I found your glasses, again... Seriously, I lost count of the many times I found your glasses at the most random places in the lab while you were walking around like a confused and scattered scientist. That being said, thank you for the time you hid $4 \mathrm{~kg}$ of fish in your carry-on luggage and for being my personal Port smuggler! I will always remember trying to impress each other with our confocal images 'look, my cell looks like Jezus...!'. Also, I will definitely see you again at the next macumba party, even though you will tell me (as usual) that you are not going. Caro Joao, muito obrigada. Imke, je talent voor guitar hero zal me altijd bijblijven (bizar), net zoals de gevaarlijke flessen vodka en je 'net iets te professionele bbq set-up'. Deze hebben menig avond onvergetelijk gemaakt, dankjewel, dzięki, tak. Irina, thank you for all the lovely dinners we had together. I'm confident to say that you make the most beautifully decorated cookies. Dear Irina, grazie mille. Dear musketeers, thank you for all the great lunches and dinners we shared along with the plenty of fun time. I really cherished our times together.

Beste Luuk, bedankt voor je 'supervisie' tijdens mijn bachelor stage (-)), en voor je kalmte in de chaos van deze PhD. Wat moet jij het af en toe moeilijk hebben gehad in een ruimte vol met hysterische vrouwen, maar je slaat je er goed doorheen. Ik heb bewondering voor hoe sterk jij jouw projecten weet te presenteren, en je openheid en je vrijgevigheid zijn mooie eigenschappen. Dear Leanne, the newest member of the crazy ladies team. Thank you for your help during my final year and the enthusiasm to take over the AV-loop project. I am sure that the AV-loop project will be a success; but remember, preparation is the key... ;-) Also, keep you wine game strong. Leanne, terima kasih. Beste kaak-afdeling, bedankt voor de leuke afdelingsuitjes en jullie enthousiasme tijdens de beer\&pizza meeting: 'als je dan toch bezig bent, kan je er dan niet ook wat neuronen en een stukje huid bij tissue engineeren?'

Mattie. Een soort van moeder (vader?) kip op het lab waar ik soms als kuikentje achter aan rende om raad. En jij op je beurt die dan zo snel mogelijk probeerde weg te rennen als je me om de hoek zag komen. Mattie, heel erg bedankt voor je oneindige steun, de ontelbare biertjes en je oprechte enthousiasme voor alle confocal plaatjes die ik schoot. Het wordt niet vaak genoeg tegen je gezegd Mattie, maar je bent echt het hart van de afdeling. Dankjewel! Dear colleagues of the biofabrication and orthopedics department, thank you so much for all the nice lunches, the conferences, the support and your big hearts while helping out each other. I have had amazing times with you at the crazy TGIFs/borrels, foodtruck festivals and lab dinners. Dear vascular-team, thank you for your input in all the presentations and discussions. Beste Petra, bedankt voor het organiseren van de vascular meetings, en je gezelschap tijdens TERMIS in Rhodos. Ik heb 
onze gesprekken altijd heel leuk gevonden, en vind jou ook zeker een zeer leuke collega! Dear Khoonoonoon, I will always remember how you introduced yourself to me: 'Hey lekkere koe', I am Khoon. Thanks to you and your chocolate from New-Zealand I definitely gained some extra weight, but having you over was always a moment I looked forward to. You always knew how to cheer me up and managed to let me see things from a different perspective. Dear sweet crazy Khoon, thanks guiding me and for teaching me so much, 谢谢. Also a big shout-out to all students that were involved in the projects: Melissa, Jonas, Bart, Myriam, Jeroen, Alexander en Arlan.

Tomasz, we managed very well to set-up a great functioning collaboration. Your work ethic and practical approach to situations are incredible. Not to mention how you always seem to be ahead of the game. I have learned a lot from you. Thank you for all the hard work, the great results and for introducing me into the magical world of the electrospinning. Also a big thanks for all the drinks, lunches and dinners in Utrecht and in Würzburg! Dear Prof. dr. Groll, thank you for the fruitful collaboration, for your hospitality and for guiding me through the process of publishing our work. Lieve Eline, wat een fijne samenwerking heb ik met jou gehad. Jouw discipline en gestructureerde manier van werken is bewonderingswaardig, en samen met jou het bioreactor project opzetten verliep mede hierdoor heel soepel. Ik denk trouwens dat wij samen een record hebben neergezet van hoe snel een paper geaccepteerd kan worden! En natuurlijk zal ik mijn tripjes met de cellen in de trein naar het 'zonnige zuiden' nooit meer vergeten. Tomasz and Eline, I could not have wished for better collaborators, and without you this thesis would not have fit together that well as it does now. Danke schön, dankjewel.

\section{Vrienden en familie/my friends and family}

Lieve Lizet. Hoe kan ik jou ooit bedanken? Waar het begon als en ontmoeting bij een PhD-event, en daarna een kopje koffie, gingen we al snel samen borrelen, thee drinken, spinnen, boksen en wandelen. Het fijn om iemand die zo begaan is met een ander aan je zij te hebben. Je staat klaar om iemand op je te laten leunen als diegene dat nodig heeft. Op tijden dat ik het even zwaar had, stond er dan weer een overlevingspakketje voor me klaar of een stukje taart. Ik kijk altijd met bewondering hoeveel druk jij aan kan, en hoe gestructureerd en efficiënt jij je door alles heen worstelt. Dankjewel voor jouw onvoorwaardelijke steun en voor alle tijd die je erin hebt gestoken om mij te helpen met mijn thesis. Let goed op jezelf, want ja 'holy shit you did it', but now'take time for yourself a bit';-).

Hola Ricardo, Mr. Tequila, Fluffy. I value every moment of our friendship, especially considering how rare it is that, even though I moved back to the Netherlands, we still see each other every year. We have shared great trips to snake/mosquito infested cottages, Belgium, Poland, Mexico and Greece, and it means the world to me that you are flying 
from Canada to Dutchland just to see my defense. Please hurry up with finding yourself a wife in Europe. Muchas gracias. Dear Sarah, my sweet Canadian girl, thank you for the deep connection, the love and hospitality of you and your family, you are 'wunderbar'.

Short, al die tijd heb jij voor me klaar gestaan, no matter what I did. Jouw wijsheden en filosofische inzichten kennen geen einde, en aan jou heb ik altijd een geweldige sparringpartner gehad om discussies mee te voeren. You are one of a kind met het hart op de goede plaats, keep your head up en volg je kompas. Ayron, bedankt voor de ontelbare bakkies koffie en je luisterend oor. Ik zie je snel weer bij de 'taxi-plaats' om een dansje te doen! Dylan, Fenna, Charley, Andy en vele andere, my personal party crew. Zonder jullie gezelligheid zou ik al veel eerder doorgedraaid zijn. Danki! Lieve maanlanding en domstad dino's, bedankt voor de steun, de gezelligheid en de dino-(lacrosse)uurtjes; vele malen hebben ze voor de welkome afleiding gezorgd.

Lief broer(tje), Max, mijn paranimf, lieve Els(a), en nu ook mijn geweldige neefje Morris. Nu kan ik mezelf eindelijk Dr. Aunt gaan noemen! Bedankt voor de vele borrels, carnavalskrakers en de oneindige spelletjes en puzzels! Lieve papa en mama. Woorden schieten tekort om jullie te bedanken. Pap, volgens mij heb jij vaker wakker gelegen dan ik over mijn papers en projecten, en was jij zenuwachtiger over naderende deadlines dan dat ik dat was. En mam, onze heerlijke wandelvakanties zijn momenten waar we allebei naar uitkijken. Of ik nu het PhD pad bewandel, of met jou in de bergen ben, overal sta je klaar om mijn blaren door te prikken. Het maakt niet uit wat ik doe, jullie staan altijd achter mij en vertrouwen daarin op mijn eigen aanpak en visie. Het geeft heel veel steun om te weten dat zolang ik gelukkig ben, jullie tevreden zijn. Jullie huis (thuis-thuis) is voor mij nog steeds een veilige haven en een welkome toevlucht naar het zonnige zuiden, waar ik altijd weer tot rust kan komen. Lieve pap en mam, bedankt, en laten we er op proosten! 

Curriculum Vitae

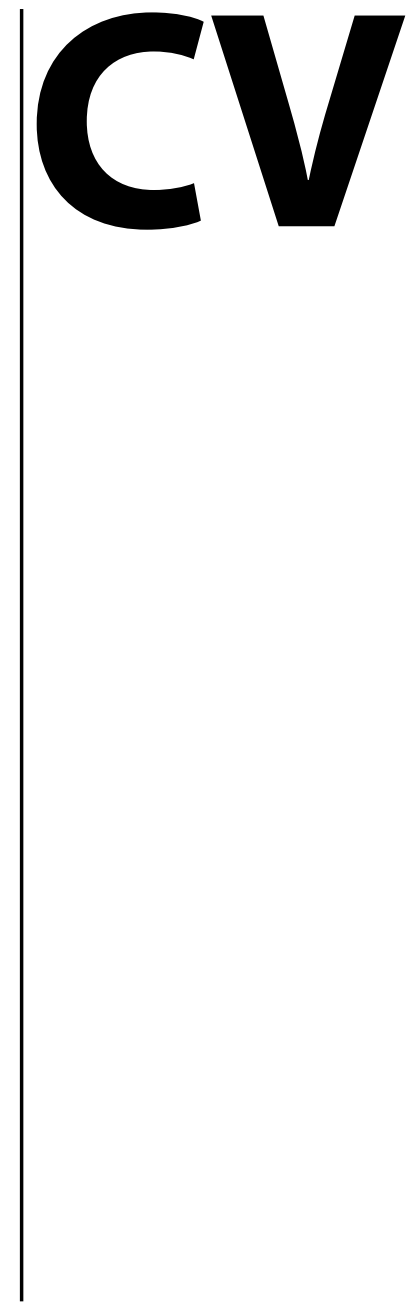




\section{Curriculum Vitae}

Iris Pennings was born on August 16th 1992 in Rosmalen, the Netherlands. In 2004 she attended secondary school (havo) at the Ds. Pierson College in 's-Hertogenbosch and continued with athenaeum in the second grade, for which she obtained her diploma in 2010. After graduation she started studying Biomedical Sciences at the Utrecht University and moved to Utrecht the following year. To finalize her bachelor's program, Iris followed the research project plus trajectory, for which she conducted an internship at the department of Orthopaedics at the University Medical Center Utrecht (UMCU) under the supervision of dr. ir. Debby Gawlitta. This internship was focused on the live-cell imaging of a co-culture of stem cells in a hydrogel system. It is safe to say that during this internship her interest for confocal imaging and regenerative medicine was sparked.

In 2013, Iris started the master's program Regenerative Medicine and Technology. During this period, she carried out her major research project at the department of Maxillofacial Surgery and Special Dental Care at the UMCU, again under the supervision of dr. ir. Debby Gawlitta. In this internship, the focus was on the vascularization of osteogenic constructs in hydrogels. In the course of this internship she got the opportunity to travel to the University of San Francisco to work on a collaborative project with MD. dr. E. Hsiao, focused on the implementation of induced pluripotent stem cells from healthy and from diseased patients (fibrodysplasia ossificans progressiva) in the hydrogel system. This collaboration later on resulted in a joint publication. Hereafter Iris wrote her literature thesis at the Eindhoven University of Technology focusing on the influence of the immune system of diabetic patients on the regeneration of heart valve scaffolds. In her final year, Iris decided to move to Toronto, Canada, to carry out her minor internship at the University of Toronto in the group of Prof. M. Sefton. In this project, driven by her fascination for vascularization, she studied this topic in combination with liver tissue engineering. By finalizing this internship, Iris obtained her Master's degree.

In 2015, she started her PhD trajectory on an NWO graduate program fellowship at the department of Maxillofacial Surgery and Special Dental Care in the Regenerative Medicine program of the UMCU, under the supervision of Prof. dr. A.J.W.P Rosenberg, Prof. dr. M.C. Verhaar, dr. ir. D. Gawlitta and dr. C. Cheng. As part of her PhD, she continued with the experimental set-up of her Master internship, and later on expanded her projects by collaborating with the University of Würzburg, Germany, and the Eindhoven University of Technology, and continued a pre-existing collaboration with the University of Zürich, Switzerland. The results of this PhD research are presented in this thesis. 



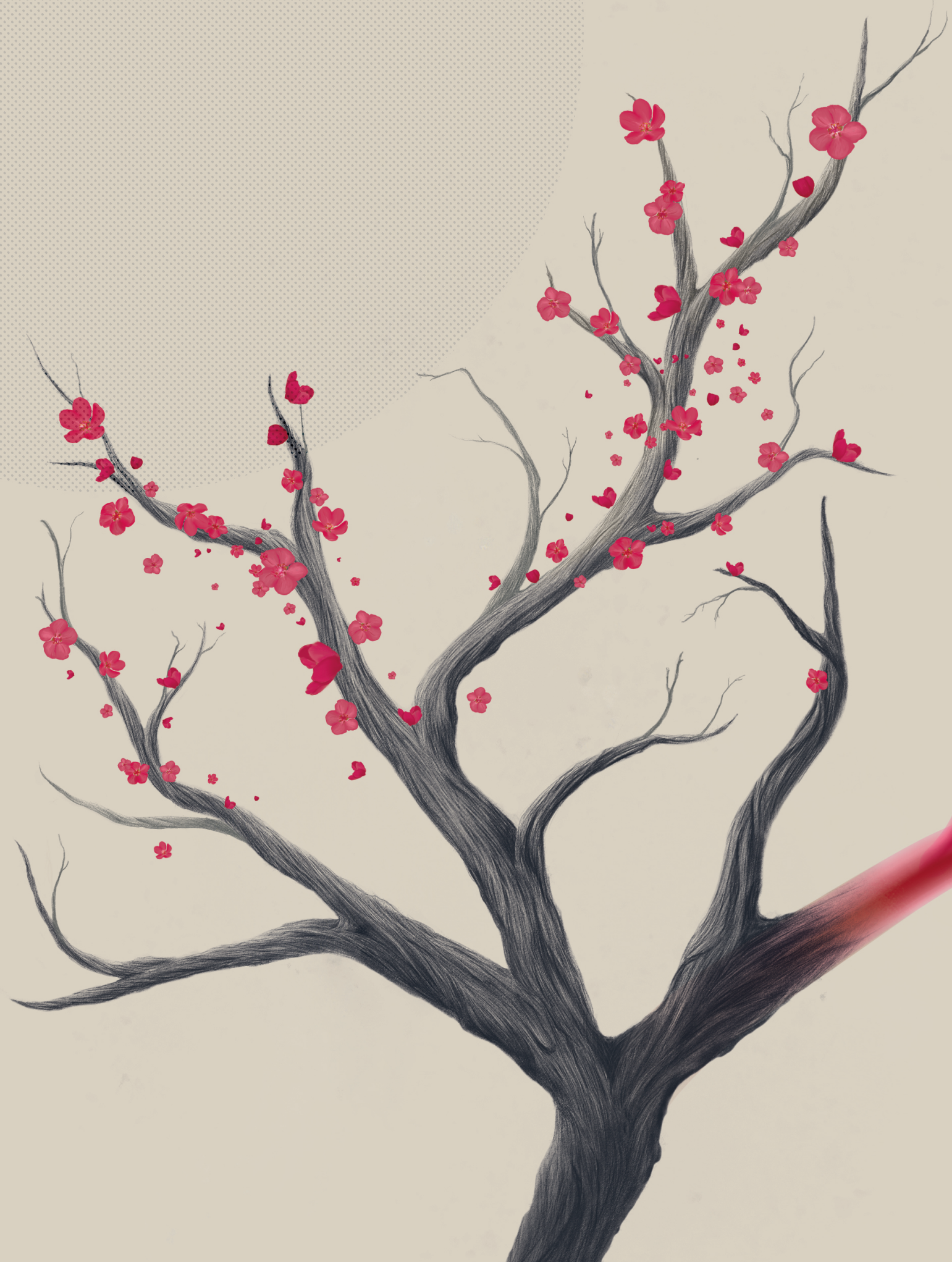

